\title{
Organoselenium-Catalyzed Synthesis of Oxygen- and Nitrogen-Containing Heterocycles
}

\author{
Ruizhi Guo, Jiachen Huang, Haiyan Huang, and Xiaodan Zhao* \\ Institute of Organic Chemistry \& MOE Key Laboratory of Bioinorganic and Synthetic Chemistry \\ School of Chemistry and Chemical Engineering, Sun Yat-Sen University, Guangzhou 510275, China
}

E-mail: zhaoxd3@mail.sysu.edu.cn

\section{Table of Contents}

1. General considerations

2. Experimental procedures

2.1 Synthesis of olefinic alcohols

2.2 Synthesis of olefinic sulfonamides

S15

2.3 Condition optimization for cyclization of olefinic alcohol 1 a

2.4 Acid-facilitated rearrangement of tetrahydrofuran $2 a$

S18

2.5 Synthesis of tetrahydrofurans or-pyrans from olefinic alcohols

S19

2.6 Condition optimization for cyclization of olefinic sulfonamide $\mathbf{4 a}$

S24

2.7 Synthesis of N-heterocycles from olefinic sulfonamides

3. Selective cyclization of olefinic alcohol 7

3.1 Synthesis of (2E,7E)-5-(hydroxymethyl)-5-phenylnona-2,7-dien-1-yl propyl carbonate (7)

3.2 Cyclization of olefinic alcohol 7 through organoselenium catalysis

3.3 Cyclization of olefinic alcohol 7 through palladium catalysis

4. Mechanistic studies

4.1 NMR studies of the equimolar mixture of PhSeSePh and 1-fluoropyridinium triflate (FP-OTf)

4.2 Synthesis of 2-phenyl-2-(3-phenyl-1-(phenylselanyl)propyl)tetrahydrofuran (10)

4.3 Reaction of new selenium species and olefinic alcohol 1a 


\section{General considerations}

Unless otherwise noted, commercial reagents were purchased from Alfa Aesar, TCI, $\mathrm{J} \& \mathrm{~K}$ or Adamas and used without further purification. THF, $\mathrm{Et}_{2} \mathrm{O}$ and toluene were dried by distillation over sodium prior to use. $\mathrm{MeCN}$, dichloromethane and 1,2-dichloroethane were dried by distillation over $\mathrm{CaH}_{2}$. All reactions were carried out using oven-dried glassware and all catalytic reactions were conducted without special care. Analytical thin layer chromatography was performed on silica gel HSGF-254 plates (Huanghai, China), and visualized under $254 \mathrm{~nm}$ UV light or by staining with potassium permanganate. Column chromatography was performed on 200-300 mesh silica gel (Huanghai, China).

${ }^{1} \mathrm{H},{ }^{19} \mathrm{~F},{ }^{13} \mathrm{C}\left\{{ }^{1} \mathrm{H}\right\}$ and ${ }^{77} \mathrm{Se}$ NMR spectra were recorded using Brucker ARX $400 \mathrm{MHz}$ spectrometer or Bruker Avance III $500 \mathrm{MHz}$ spectrometer at ambient temperature. All NMR spectra are referenced to the residual solvent signal $\left(\mathrm{CHCl}_{3}: \delta \mathrm{H}=7.26\right.$ and $\delta \mathrm{C}$ $=77.16 ; \mathrm{MeCN}: \delta \mathrm{H}=1.94)$. Data for ${ }^{1} \mathrm{H}$ NMR are reported as follows: chemical shift $(\delta \mathrm{ppm})$, multiplicity $(\mathrm{s}=$ singlet, $\mathrm{d}=$ doublet, $\mathrm{t}=$ triplet, $\mathrm{q}=$ quartet, $\mathrm{m}=$ multiplet, $\mathrm{br}$ = broad), coupling constant $(\mathrm{Hz})$, integration. Data for ${ }^{13} \mathrm{C}\left\{{ }^{1} \mathrm{H}\right\}$ NMR are reported as follows: chemical shift $(\delta \mathrm{ppm})$, multiplicity $(\mathrm{d}=$ doublet, $\mathrm{t}=$ triplet, $\mathrm{q}=$ quartet $)$, coupling constant $(\mathrm{Hz})$.

High resolution mass spectra (HRMS) were recorded on Thermo MAT95XP mass spectrometer at analytical center of Sun Yat-Sen University or Bruker Agilent 1290 mass spectrometer at analytical center of South China University of Technology. 


\section{Experimental procedures}

\subsection{Synthesis of olefinic alcohols}

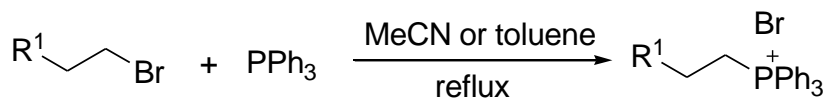

General procedure for synthesis of triphenylphosphonium bromide: In an oven-dried $50 \mathrm{~mL}$ Schlenk flask equipped with a stir bar and Graham condenser was added $\mathrm{PPh}_{3}$ ( $3.15 \mathrm{~g}, 12 \mathrm{mmol})$. The flask was then evacuated and back-filled with nitrogen. Next, bromide $(12 \mathrm{mmol})$ in anhydrous $\mathrm{MeCN}$ or toluene $(10 \mathrm{~mL})$ was subsequently added via syringe. The homogeneous mixture was stirred at reflux for $62 \mathrm{~h}$. The resulting colorless solution was cooled to room temperature and then $\mathrm{Et}_{2} \mathrm{O}(20 \mathrm{~mL})$ was added. Keeping static for a while, the formed precipitate was filtered, washed with $\mathrm{Et}_{2} \mathrm{O}$ (20 $\mathrm{mL} \times 3$ ), and dried in vacuo to afford the triphenylphosphonium bromide as a white solid.

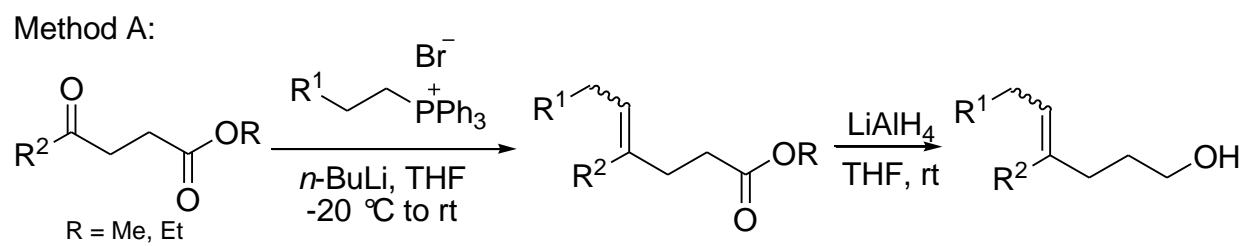

General procedure A for the synthesis of substrates $\mathbf{1 a}, \mathbf{1 b}, \mathbf{1 c}, \mathbf{1 d}$, and $\mathbf{1 f}$.

An oven-dried $50 \mathrm{~mL}$ Schlenk flask equipped with a stir bar was charged with triphenylphosphonium bromide (12 mmol). The flask was then evacuated and back-filled with nitrogen. Then anhydrous THF $(20 \mathrm{~mL})$ was added via syringe and the suspension was cooled to $-20^{\circ} \mathrm{C}$. To the colorless suspension was added $n$-BuLi (2.5 $\mathrm{M}$ in hexane) $(5 \mathrm{~mL}, 12.5 \mathrm{mmol})$ dropwise via syringe over $5 \mathrm{~min}$. The resulting orange solution was stirred at $-20^{\circ} \mathrm{C}$ for $30 \mathrm{~min}$ and became homogeneous gradually. Then a solution of keto ester $(10 \mathrm{mmol})$ in anhydrous THF $(2 \mathrm{~mL})$ was added via syringe at $-20^{\circ} \mathrm{C}$. The resulting mixture was warmed to room temperature gradually and stirred overnight, after which the heterogeneous mixture was quenched with saturated aqueous $\mathrm{NH}_{4} \mathrm{Cl}$ solution $(60 \mathrm{~mL})$ and extracted with EtOAc $(60 \mathrm{~mL} \times 3)$. The organic extracts were dried over anhydrous $\mathrm{Na}_{2} \mathrm{SO}_{4}$, filtered and concentrated in vacuo. The residue was purified by silica gel column chromatography to afford the olefinic ester.

An oven-dried $25 \mathrm{~mL}$ Schlenk flask equipped with a stir bar was charged with $\mathrm{LiAlH}_{4}$ (114 mg, $3 \mathrm{mmol}$ ). The flask was then evacuated and back-filled with nitrogen. Then anhydrous THF ( $7 \mathrm{~mL})$ was added via syringe, after which a solution of olefinc ester ( $2 \mathrm{mmol}$ ) in anhydrous THF ( $3 \mathrm{~mL}$ ) was added to the suspension at room temperature over $5 \mathrm{~min}$. After stirred at room temperature for $3 \mathrm{~h}$, the resulting heterogeneous mixture was quenched with $\mathrm{H}_{2} \mathrm{O}(200 \mu \mathrm{L})$ and aqueous $10 \% \mathrm{NaOH}$ solution $(200 \mu \mathrm{L})$ carefully. After stirred at room temperature for additional $2 \mathrm{~h}$, the resulting mixture was dried over anhydrous $\mathrm{Na}_{2} \mathrm{SO}_{4}$, filtered and concentrated in vacuo. The residue 
was purified by silica gel column chromatography to afford the olefinic alcohol.<smiles></smiles>

4,7-Diphenylhept-4-en-1-ol (1a) ( $E / Z=4: 1)$ : Prepared according to general procedure A. Step 1: Flash column chromatography (eluent: PE/EtOAc $=60: 1, \mathrm{v} / \mathrm{v}$ ) to afford olefinic ester $(\mathrm{R}=\mathrm{Me})(1.23 \mathrm{~g}, 42 \%)$ as a yellow oil. Step 2: Flash column chromatography (eluent: $\mathrm{PE} / \mathrm{EtOAc}=6: 1, \mathrm{v} / \mathrm{v})$ to afford $1 \mathrm{a}(522 \mathrm{mg}, 98 \%)$ as a colorless oil. The ${ }^{1} \mathrm{H}$ NMR spectrum was in agreement with that reported in the reference. ${ }^{1} E$-isomer, ${ }^{1} \mathrm{H}$ NMR $\left(400 \mathrm{MHz}, \mathrm{CDCl}_{3}\right) \delta 7.35-7.14(\mathrm{~m}, 6 \mathrm{H}), 7.10(\mathrm{~d}, J=$ $6.8 \mathrm{~Hz}, 2 \mathrm{H}), 7.02(\mathrm{~d}, J=6.8 \mathrm{~Hz}, 2 \mathrm{H}), 5.51(\mathrm{t}, J=7.3 \mathrm{~Hz}, 1 \mathrm{H}), 3.59(\mathrm{t}, J=6.5 \mathrm{~Hz}, 2 \mathrm{H})$, $2.68-2.59(\mathrm{~m}, 2 \mathrm{H}), 2.47-2.36(\mathrm{~m}, 2 \mathrm{H}), 2.25(\mathrm{dd}, J=15.1,7.5 \mathrm{~Hz}, 2 \mathrm{H}), 1.59-1.47$ $(\mathrm{m}, 2 \mathrm{H})$.<smiles>CC/C=C(\CCCO)c1ccccc1</smiles>

4-Phenyloct-4-en-1-ol (1b) $(E / Z=4: 1)$ : Prepared according to general procedure A. Step 1: Flash column chromatography (eluent: PE/EtOAc $=60: 1, \mathrm{v} / \mathrm{v}$ ) to afford olefinic ester $(\mathrm{R}=\mathrm{Me})(927 \mathrm{mg}, 40 \%)$ as a yellow oil. Step 2: Flash column chromatography (eluent: $\mathrm{PE} / \mathrm{EtOAc}=6: 1, \mathrm{v} / \mathrm{v})$ to afford $\mathbf{1 b}(371 \mathrm{mg}, 91 \%)$ as a colorless oil. E-isomer, ${ }^{1} \mathrm{H}$ NMR $\left(400 \mathrm{MHz}, \mathrm{CDCl}_{3}\right) \delta 7.32(\mathrm{t}, J=6.5 \mathrm{~Hz}, 2 \mathrm{H}), 7.24(\mathrm{t}$, $J=7.3 \mathrm{~Hz}, 1 \mathrm{H}), 7.14(\mathrm{~d}, J=6.9 \mathrm{~Hz}, 2 \mathrm{H}), 5.49(\mathrm{t}, J=7.3 \mathrm{~Hz}, 1 \mathrm{H}), 3.62(\mathrm{t}, J=6.5 \mathrm{~Hz}$, $2 \mathrm{H}), 2.43(\mathrm{t}, J=7.3 \mathrm{~Hz}, 2 \mathrm{H}), 1.91(\mathrm{q}, J=7.4 \mathrm{~Hz}, 2 \mathrm{H}), 1.61-1.54(\mathrm{~m}, 2 \mathrm{H}), 1.40-$ $1.28(\mathrm{~m}, 2 \mathrm{H}), 0.84(\mathrm{t}, J=7.4 \mathrm{~Hz}, 3 \mathrm{H}) .{ }^{13} \mathrm{C} \mathrm{NMR}\left(101 \mathrm{MHz}, \mathrm{CDCl}_{3}\right) \delta 141.2,140.4$, 128.6, 128.2, 128.0, 126.6, 62.7, 35.6, 31.3, 31.1, 23.3, 13.9. HR-ESI-MS m/z calcd for $\mathrm{C}_{12} \mathrm{H}_{20} \mathrm{O}[\mathrm{M}+\mathrm{H}]^{+}:$205.1587, found: 205.1589 .<smiles>CCCCCC=C(CCCO)c1ccccc1</smiles>

4-Phenyldec-4-en-1-ol (1c) $(E / Z=4: 1)$ : Prepared according to general procedure A. Step 1: Flash column chromatography (eluent: PE/EtOAc $=60: 1, \mathrm{v} / \mathrm{v}$ ) to afford olefinic ester $(\mathrm{R}=\mathrm{Me})(862 \mathrm{mg}, 33 \%)$ as a yellow oil. Step 2: Flash column chromatography (eluent: PE/EtOAc $=6: 1, \mathrm{v} / \mathrm{v})$ to afford 1c $(411 \mathrm{mg}, 88 \%)$ as a colorless oil. $E$-isomer, ${ }^{1} \mathrm{H}$ NMR $\left(400 \mathrm{MHz}, \mathrm{CDCl}_{3}\right) \delta 7.32(\mathrm{t}, J=6.4 \mathrm{~Hz}, 2 \mathrm{H}), 7.23(\mathrm{t}$, $J=7.3 \mathrm{~Hz}, 1 \mathrm{H}), 7.14(\mathrm{~d}, J=6.8 \mathrm{~Hz}, 2 \mathrm{H}), 5.48(\mathrm{t}, J=7.3 \mathrm{~Hz}, 1 \mathrm{H}), 3.69-3.54(\mathrm{~m}, 2 \mathrm{H})$, $2.42(\mathrm{t}, J=7.5 \mathrm{~Hz}, 2 \mathrm{H}), 1.92(\mathrm{q}, J=7.3 \mathrm{~Hz}, 2 \mathrm{H}), 1.64-1.50(\mathrm{~m}, 2 \mathrm{H}), 1.40-1.13(\mathrm{~m}$, $6 \mathrm{H}), 0.84(\mathrm{t}, J=6.9 \mathrm{~Hz}, 3 \mathrm{H}) .{ }^{13} \mathrm{C}$ NMR $\left(101 \mathrm{MHz}, \mathrm{CDCl}_{3}\right) \delta 141.2,140.2,128.6$, 128.2, 128.2, 126.6, 62.7, 35.6, 31.6, 31.3, 29.9, 29.0, 22.7, 14.2. HR-ESI-MS m/z calcd for $\mathrm{C}_{16} \mathrm{H}_{24} \mathrm{O}[\mathrm{M}+\mathrm{H}]^{+}: 233.1900$, found: 233.1897 .<smiles>C/C(=C/CCc1ccccc1)CCCO</smiles>

4-Methyl-7-phenylhept-4-en-1-ol (1d) $(E / Z=2: 1)$ : Prepared according to general procedure A. Step 1: Flash column chromatography (eluent: PE/EtOAc $=60: 1, \mathrm{v} / \mathrm{v}$ ) to 
afford olefinic ester $(\mathrm{R}=\mathrm{Et})(769 \mathrm{mg}, 31 \%)$ as a colorless oil. Step 2: Flash column chromatography (eluent: PE/EtOAc $=6: 1, \mathrm{v} / \mathrm{v})$ to afford 1d $(392 \mathrm{mg}, 96 \%)$ as a colorless oil. The ${ }^{1} \mathrm{H}$ NMR spectrum was in agreement with those reported in the reference. ${ }^{2} E$-isomer, ${ }^{1} \mathrm{H}$ NMR $\left(400 \mathrm{MHz}, \mathrm{CDCl}_{3}\right) \delta 7.29(\mathrm{~d}, J=8.0 \mathrm{~Hz}, 2 \mathrm{H}), 7.19(\mathrm{~d}$, $J=6.5 \mathrm{~Hz}, 3 \mathrm{H}), 5.22(\mathrm{t}, J=7.1 \mathrm{~Hz}, 1 \mathrm{H}), 3.57(\mathrm{t}, J=6.5 \mathrm{~Hz}, 2 \mathrm{H}), 2.64(\mathrm{t}, J=7.8 \mathrm{~Hz}$, $2 \mathrm{H}), 2.32(\mathrm{q}, J=7.5 \mathrm{~Hz}, 2 \mathrm{H}), 2.09-2.01(\mathrm{~m}, 2 \mathrm{H}), 1.69(\mathrm{~s}, 2 \mathrm{H}), 1.69-1.59(\mathrm{~m}, 2 \mathrm{H})$, $1.56(\mathrm{~s}, 3 \mathrm{H})$.<smiles>C/C(=C/CCCCO)CCCCCCCO</smiles>

10-(Benzyloxy)-4-methyldec-4-en-1-ol (1f) $(E / Z=2: 1)$ : Prepared according to general procedure A. Step 1: Flash column chromatography (eluent: $\mathrm{PE} / \mathrm{EtOAc}=40: 1$ to $\mathrm{PE} / \mathrm{EtOAc}=20: 1, \mathrm{v} / \mathrm{v})$ to afford olefinic ester $(\mathrm{R}=\mathrm{Et})(482 \mathrm{mg}, 15 \%)$ as a colorless oil. Step 2: Flash column chromatography (eluent: $\mathrm{PE} / \mathrm{EtOAc}=5: 1, \mathrm{v} / \mathrm{v}$ ) to afford 1f $(492 \mathrm{mg}, 89 \%)$ as a colorless oil. $E$-isomer, ${ }^{1} \mathrm{H} \mathrm{NMR}\left(400 \mathrm{MHz}, \mathrm{CDCl}_{3}\right) \delta$ $7.37-7.27(\mathrm{~m}, 5 \mathrm{H}), 5.15(\mathrm{t}, J=6.8 \mathrm{~Hz}, 1 \mathrm{H}), 4.50(\mathrm{~s}, 2 \mathrm{H}), 3.61(\mathrm{t}, J=6.4 \mathrm{~Hz}, 2 \mathrm{H})$, $3.47(\mathrm{t}, J=6.6 \mathrm{~Hz}, 2 \mathrm{H}), 2.13-1.95(\mathrm{~m}, 4 \mathrm{H}), 1.73-1.59(\mathrm{~m}, 7 \mathrm{H}), 1.41-1.32(\mathrm{~m}, 4 \mathrm{H})$. ${ }^{13} \mathrm{C}$ NMR $\left(101 \mathrm{MHz}, \mathrm{CDCl}_{3}\right) \delta 138.7,134.8,128.5,127.8,127.6,126.0,73.0,70.6$, 63.0, 31.0, 23.0, 29.8, 28.1, 27.8, 26.0, 23.5. HR-ESI-MS m/z calcd for $\mathrm{C}_{18} \mathrm{H}_{28} \mathrm{O}_{2}$ $[\mathrm{M}+\mathrm{H}]^{+}:$277.2162, found: 277.2167 .

Method B:
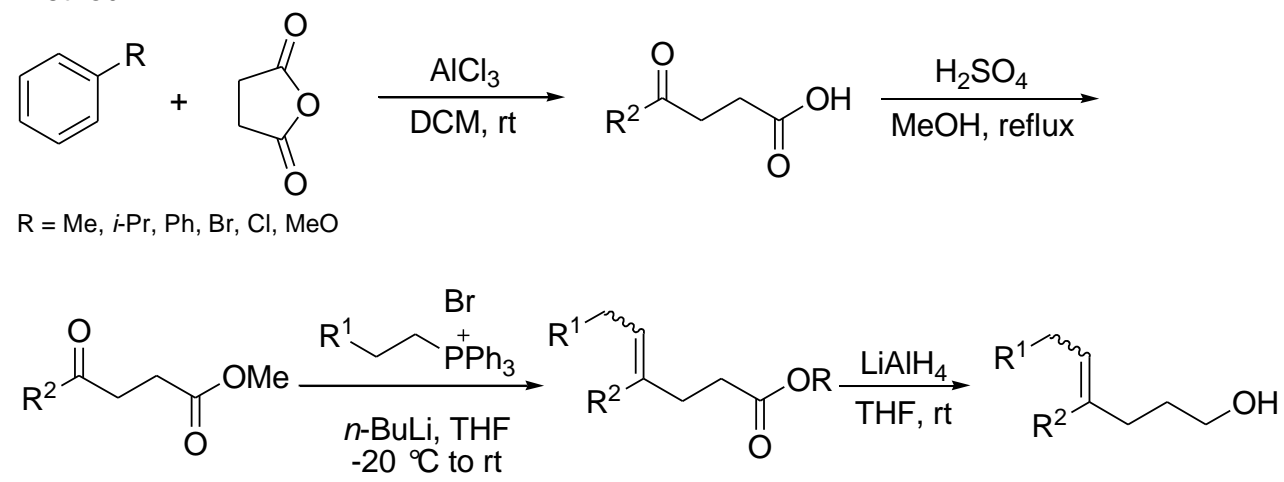

General procedure B for the synthesis of substrates $\mathbf{1 h}, \mathbf{1} \mathbf{j}, \mathbf{1}, \mathbf{1} \mathbf{m}, \mathbf{1 n}$ and $\mathbf{1 0 .}$

An oven-dried $100 \mathrm{~mL}$ Schlenk flask was equipped with a stir bar and moved into glovebox. Then, succinic anhydride $(2.0 \mathrm{~g}, 20 \mathrm{mmol})$ and anhydrous $\mathrm{AlCl}_{3}(5.87 \mathrm{~g}, 44$ mmol) was subsequently added. The flask was sealed with a Septum and removed from the glovebox. Anhydrous DCM (40 mL) was then added via syringe and the suspension was stirred at room temperature for $5 \mathrm{~min}$. Then, the flask was charged with a $\mathrm{HCl}$ gas absorption device and to the pale yellow heterogeneous mixture was added arene via syringe at room temperature over $10 \mathrm{~min}$ with observation of $\mathrm{HCl}$ gas evolution. The mixture was allowed to stir at room temperature overnight and became homogeneous gradually. The resulting mixture was quenched with aqueous $1 \mathrm{M} \mathrm{HCl}$ solution $(30 \mathrm{~mL})$ carefully. Then, the mixture was diluted with $\mathrm{Et}_{2} \mathrm{O}(80 \mathrm{~mL})$ and washed with aqueous $1 \mathrm{M} \mathrm{HCl}$ solution $(80 \mathrm{~mL} \times 3)$. The organic phase was concentrated in vacuo. The crude product was dissolved in aqueous $1 \mathrm{M} \mathrm{NaOH}$ 
solution $(40 \mathrm{~mL})$ and washed with $\mathrm{DCM}(40 \mathrm{~mL} \times 3)$. The aqueous phase was acidified by aqueous $1 \mathrm{M} \mathrm{HCl}$ solution $(45 \mathrm{~mL})$. The formed precipitate was filtered by Büchner funnel and dried in air overnight to afford the keto acid as a white solid.

To a $100 \mathrm{~mL}$ round-bottom flask equipped with a stir bar and Graham condenser was added keto acid (18 mmol) and $\mathrm{MeOH}(0.5 \mathrm{M}$ solution). Then, concentrated sulphuric acid $(2 \mathrm{~mL})$ was added dropwise. After stirred at reflux for $18 \mathrm{~h}$, the resulting mixture was quenched with saturated aqueous $\mathrm{NaHCO}_{3}$ solution $(20 \mathrm{~mL})$ carefully and stirred for additional $1 \mathrm{~h}$. The resulting biphasic mixture was extracted with DCM $(25 \mathrm{~mL} \times$ 3 ). The organic extracts were dried over anhydrous $\mathrm{Na}_{2} \mathrm{SO}_{4}$, filtered and concentrated in vacuo. The residue was purified by silica gel column chromatography to afford the keto ester.

The synthesis of olefinic ester and olefinic alcohol is similar to the general procedure A.<smiles>CCCC=C(CCCO)c1ccc(C)cc1</smiles>

4-(P-tolyl)oct-4-en-1-ol (1h) $(E / Z=3: 1)$ : Prepared according to general procedure B. Step 1: Afford keto acid (3.81 g, 99\%) as a white solid. Step 2: Flash column chromatography (eluent: $\mathrm{PE} / \mathrm{EtOAc}=15: 1, \mathrm{v} / \mathrm{v})$ to afford keto ester $(3.25 \mathrm{~g}, 88 \%)$ as a white solid. Step 3: Flash column chromatography (eluent: PE/EtOAc $=60: 1, \mathrm{v} / \mathrm{v}$ ) to afford olefinic ester $(640 \mathrm{mg}, 26 \%)$ as a yellow oil. Step 4: Flash column chromatography (eluent: $\mathrm{PE} / \mathrm{EtOAc}=6: 1, \mathrm{v} / \mathrm{v})$ to afford $\mathbf{1 h}(380 \mathrm{mg}, 87 \%)$ as a colorless oil. $E$-isomer, ${ }^{1} \mathrm{H}$ NMR $\left(400 \mathrm{MHz}, \mathrm{CDCl}_{3}\right) \delta 7.14(\mathrm{~d}, J=7.8 \mathrm{~Hz}, 2 \mathrm{H}), 7.04$ $(\mathrm{d}, J=8.0 \mathrm{~Hz}, 2 \mathrm{H}), 5.46(\mathrm{t}, J=7.3 \mathrm{~Hz}, 1 \mathrm{H}), 3.61(\mathrm{t}, J=6.5 \mathrm{~Hz}, 2 \mathrm{H}), 2.41(\mathrm{t}, J=7.5$ $\mathrm{Hz}, 2 \mathrm{H}), 2.35$ (s, 3H), $1.92(\mathrm{q}, J=7.3 \mathrm{~Hz}, 2 \mathrm{H}), 1.61-1.53(\mathrm{~m}, 2 \mathrm{H}), 1.40-1.28(\mathrm{~m}$, $2 \mathrm{H}), 0.84(\mathrm{t}, J=7.4 \mathrm{~Hz}, 3 \mathrm{H}) .{ }^{13} \mathrm{C}$ NMR $\left(101 \mathrm{MHz}, \mathrm{CDCl}_{3}\right) \delta 140.2,138.1,136.1$, 129.1, 128.9, 128.5, 62.7, 35.7, 31.3, 31.1, 23.4, 21.3, 13.9. HR-ESI-MS m/z calcd for $\mathrm{C}_{15} \mathrm{H}_{22} \mathrm{O}[\mathrm{M}+\mathrm{H}]^{+}:$219.1743, found: 219.1746 .<smiles>CCCC=C(CCCO)c1ccc(C(C)C)cc1</smiles>

4-(4-Isopropylphenyl)oct-4-en-1-ol $(\mathbf{1 j})(E / Z=4: 1)$ : Prepared according to general procedure B. Step 1: Afford keto acid $(3.57 \mathrm{~g}, 81 \%)$ as a white solid. Step 2: Flash column chromatography (eluent: $\mathrm{PE} / \mathrm{EtOAc}=15: 1, \mathrm{v} / \mathrm{v})$ to afford keto ester $(3.08 \mathrm{~g}$, $73 \%$ ) as a white solid. Step 3: Flash column chromatography (eluent: PE/EtOAc = $60: 1, \mathrm{v} / \mathrm{v})$ to afford olefinic ester $(478 \mathrm{mg}, 17 \%)$ as a yellow oil. Step 4: Flash column chromatography (eluent: $\mathrm{PE} / \mathrm{EtOAc}=6: 1, \mathrm{v} / \mathrm{v})$ to afford $\mathbf{1 j}(418 \mathrm{mg}, 85 \%)$ as a colorless oil. $E$-isomer, ${ }^{1} \mathrm{H}$ NMR $\left(400 \mathrm{MHz}, \mathrm{CDCl}_{3}\right) \delta 7.17(\mathrm{~d}, J=8.0 \mathrm{~Hz}, 2 \mathrm{H}), 7.06$ $(\mathrm{d}, J=8.1 \mathrm{~Hz}, 2 \mathrm{H}), 5.46(\mathrm{t}, J=7.3 \mathrm{~Hz}, 1 \mathrm{H}), 3.61(\mathrm{t}, J=5.9 \mathrm{~Hz}, 2 \mathrm{H}), 2.96-2.85(\mathrm{~m}$, $1 \mathrm{H}), 2.41(\mathrm{t}, J=7.3 \mathrm{~Hz}, 2 \mathrm{H}), 1.94(\mathrm{q}, J=7.3 \mathrm{~Hz}, 2 \mathrm{H}), 1.63-1.53(\mathrm{~m}, 2 \mathrm{H}), 1.40-$ 
$1.29(\mathrm{~m}, 2 \mathrm{H}), 1.26(\mathrm{~d}, J=6.9 \mathrm{~Hz}, 6 \mathrm{H}), 0.85(\mathrm{t}, J=7.4 \mathrm{~Hz}, 3 \mathrm{H}) .{ }^{13} \mathrm{C}$ NMR $(101 \mathrm{MHz}$, $\left.\mathrm{CDCl}_{3}\right) \delta 147.0,140.2,138.4,128.4,127.7,126.2,62.8,35.7,33.9,31.4,31.1,24.1$, 23.4, 13.9. HR-ESI-MS m/z calcd for $\mathrm{C}_{17} \mathrm{H}_{26} \mathrm{O}[\mathrm{M}+\mathrm{H}]^{+}: 247.2056$, found: 247.2060 .<smiles>CCCC=C(CCCO)c1ccc(Cl)cc1</smiles>

4-(4-Chlorophenyl)oct-4-en-1-ol (11) $(E / Z=2: 1)$ : Prepared according to general procedure B. Step 1: Afford keto acid $(3.81 \mathrm{~g}, 90 \%)$ as a pale orange solid. Step 2: Flash column chromatography (eluent: PE/EtOAc $=15: 1, \mathrm{v} / \mathrm{v}$ ) to afford keto ester (3.83 g, 94\%) as an orange solid. Step 3: Flash column chromatography (eluent: $\mathrm{PE} / \mathrm{EtOAc}=70: 1, \mathrm{v} / \mathrm{v})$ to afford olefinic ester $(727 \mathrm{mg}, 27 \%)$ as a yellow oil. Step 4: Flash column chromatography (eluent: $\mathrm{PE} / \mathrm{EtOAc}=6: 1, \mathrm{v} / \mathrm{v})$ to afford 11 (442 $\mathrm{mg}$, $93 \%)$ as a colorless oil. $E$-isomer, ${ }^{1} \mathrm{H} \mathrm{NMR}\left(400 \mathrm{MHz}, \mathrm{CDCl}_{3}\right) \delta 7.29(\mathrm{~d}, J=8.4 \mathrm{~Hz}$, 2H), $7.06(\mathrm{~d}, J=8.4 \mathrm{~Hz}, 2 \mathrm{H}), 5.49(\mathrm{t}, J=7.4 \mathrm{~Hz}, 1 \mathrm{H}), 3.61(\mathrm{t}, J=4.9 \mathrm{~Hz}, 2 \mathrm{H}), 2.39(\mathrm{t}$, $J=7.4 \mathrm{~Hz}, 2 \mathrm{H}), 1.88(\mathrm{q}, J=7.4 \mathrm{~Hz}, 2 \mathrm{H}), 1.62-1.53(\mathrm{~m}, 2 \mathrm{H}), 1.39-1.29(\mathrm{~m}, 2 \mathrm{H})$, $0.83(\mathrm{t}, J=7.4 \mathrm{~Hz}, 3 \mathrm{H}) .{ }^{13} \mathrm{C} \mathrm{NMR}\left(101 \mathrm{MHz}, \mathrm{CDCl}_{3}\right) \delta 139.6,139.3,132.4,130.0$, 128.5, 128.4, 62.6, 35.5, 31.2, 31.1, 23.3, 13.9. HR-ESI-MS m/z calcd for $\mathrm{C}_{14} \mathrm{H}_{19} \mathrm{ClO}$ $[\mathrm{M}+\mathrm{Na}]^{+}: 261.1017$, found: 261.1013 .<smiles>CCCC=C(CCCO)c1ccc(Br)cc1</smiles>

4-(4-Bromophenyl)oct-4-en-1-ol $(\mathbf{1 m})(E / Z=2: 1)$ : Prepared according to general procedure B. Step 1: Afford keto acid (4.23 g, 82\%) as a pale orange solid. Step 2: Flash column chromatography (eluent: PE/EtOAc $=6: 1, \mathrm{v} / \mathrm{v}$ ) to afford keto ester (4.37 g, 90\%) as an orange solid. Step 3: Flash column chromatography (eluent: $\mathrm{PE} / \mathrm{EtOAc}=70: 1, \mathrm{v} / \mathrm{v})$ to afford olefinic ester $(538 \mathrm{mg}, 17 \%)$ as a yellow oil. Step 4: Flash column chromatography (eluent: PE/EtOAc $=8: 1, \mathrm{v} / \mathrm{v})$ to afford $\mathbf{1 m}(352 \mathrm{mg}$, $62 \%)$ as a colorless oil. $E$-isomer, ${ }^{1} \mathrm{H} \mathrm{NMR}\left(400 \mathrm{MHz}, \mathrm{CDCl}_{3}\right) \delta 7.45(\mathrm{~d}, J=8.5 \mathrm{~Hz}$, $2 \mathrm{H}), 7.01(\mathrm{~d}, J=8.4 \mathrm{~Hz}, 2 \mathrm{H}), 5.50(\mathrm{t}, J=7.4 \mathrm{~Hz}, 1 \mathrm{H}), 3.61(\mathrm{t}, J=6.5 \mathrm{~Hz}, 2 \mathrm{H}), 2.40(\mathrm{t}$, $J=7.2 \mathrm{~Hz}, 2 \mathrm{H}), 1.88(\mathrm{q}, J=7.4 \mathrm{~Hz}, 2 \mathrm{H}), 1.63-1.51(\mathrm{~m}, 2 \mathrm{H}), 1.38-1.27(\mathrm{~m}, 2 \mathrm{H})$, $0.83(\mathrm{t}, J=7.4 \mathrm{~Hz}, 3 \mathrm{H}) .{ }^{13} \mathrm{C} \mathrm{NMR}\left(101 \mathrm{MHz}, \mathrm{CDCl}_{3}\right) \delta 140.1,139.2,131.3,130.3$, 128.1, 120.5, 62.5, 35.4, 31.2, 31.0, 23.2, 13.9. HR-EI-MS m/z calcd for $\mathrm{C}_{14} \mathrm{H}_{19} \mathrm{BrO}$ [M]: 282.0619, found: 282.0616 .<smiles>CCCC=C(CCCO)c1ccc(-c2ccccc2)cc1</smiles>

4-([1,1'-Biphenyl]-4-yl)oct-4-en-1-ol (1n) $(E / Z=2: 1)$ : Prepared according to general procedure B. Step 1: Afford keto acid (5.03 g, 99\%) as a yellow solid. Step 2: Flash column chromatography (eluent: PE/EtOAc $=10: 1, \mathrm{v} / \mathrm{v})$ to afford keto ester $(3.37 \mathrm{~g}$, $70 \%$ ) as a yellow solid. Step 3: Flash column chromatography (eluent: PE/EtOAc $=$ $70: 1$, v/v) to afford olefinic ester (564 $\mathrm{mg}, 18 \%$ ) as a yellow oil. Step 4: Flash column 
chromatography (eluent: PE/EtOAc $=6: 1, \mathrm{v} / \mathrm{v})$ to afford 1n $(534 \mathrm{mg}, 95 \%)$ as a white solid. E-isomer, ${ }^{1} \mathrm{H}$ NMR (400 MHz, $\left.\mathrm{CDCl}_{3}\right) \delta 7.66-7.53(\mathrm{~m}, 4 \mathrm{H}), 7.49-7.41(\mathrm{~m}$, $3 \mathrm{H}), 7.38-7.31(\mathrm{~m}, 1 \mathrm{H}), 7.26-7.20(\mathrm{~m}, 1 \mathrm{H}), 5.54(\mathrm{t}, J=6.8 \mathrm{~Hz}, 1 \mathrm{H}), 3.65(\mathrm{t}, J=6.5$ $\mathrm{Hz}, 2 \mathrm{H}), 2.48(\mathrm{t}, J=7.4 \mathrm{~Hz}, 2 \mathrm{H}), 2.05-1.94(\mathrm{~m}, 2 \mathrm{H}), 1.72-1.57(\mathrm{~m}, 2 \mathrm{H}), 1.45-$ $1.32(\mathrm{~m}, 2 \mathrm{H}), 0.88(\mathrm{t}, J=7.5 \mathrm{~Hz}, 3 \mathrm{H}) .{ }^{13} \mathrm{C} \mathrm{NMR}\left(101 \mathrm{MHz}, \mathrm{CDCl}_{3}\right) \delta 142.1,141.1$, 140.2, 140.0, 139.4, 129.1, 129.0, 127.3, 127.1, 126.9, 62.7, 35.6, 31.4, 31.2, 23.4, 14.0. HR-ESI-MS m/z calcd for $\mathrm{C}_{20} \mathrm{H}_{24} \mathrm{O}[\mathrm{M}+\mathrm{H}]^{+}: 281.1900$, found: 281.1904 .<smiles>C/C=C(/CCCO)c1ccc(-c2ccccc2)cc1</smiles>

4-([1,1'-Biphenyl]-4-yl)hex-4-en-1-ol (1o) $(E / Z=3: 1)$ : Prepared according to general procedure B. Step 3: Flash column chromatography (eluent: $\mathrm{PE} / \mathrm{EtOAc}=40: 1, \mathrm{v} / \mathrm{v}$ ) to afford olefinic ester $(1.65 \mathrm{~g}, 59 \%)$ as a yellow oil. Step 4: Flash column chromatography (eluent: $\mathrm{PE} / \mathrm{EtOAc}=4: 1, \mathrm{v} / \mathrm{v})$ to afford $10(333 \mathrm{mg}, 66 \%)$ as a pale yellow solid. $E$-isomer, ${ }^{1} \mathrm{H} \mathrm{NMR}\left(400 \mathrm{MHz}, \mathrm{CDCl}_{3}\right) \delta 7.66-7.53(\mathrm{~m}, 4 \mathrm{H}), 7.48-$ $7.40(\mathrm{~m}, 3 \mathrm{H}), 7.35(\mathrm{t}, J=6.8 \mathrm{~Hz}, 1 \mathrm{H}), 7.24(\mathrm{~d}, J=7.9 \mathrm{~Hz}, 1 \mathrm{H}), 5.63(\mathrm{q}, J=6.7 \mathrm{~Hz}$, $1 \mathrm{H}), 3.64(\mathrm{t}, J=6.4 \mathrm{~Hz}, 2 \mathrm{H}), 2.48(\mathrm{t}, J=7.5 \mathrm{~Hz}, 2 \mathrm{H}), 1.72-1.57(\mathrm{~m}, 5 \mathrm{H}) .{ }^{13} \mathrm{C} \mathrm{NMR}$ $\left(101 \mathrm{MHz}, \mathrm{CDCl}_{3}\right) \delta 142.0,141.1,140.7,139.8,139.4,129.1,128.9,127.1,126.9$, 122.1, 62.7, 35.4, 31.4, 14.9. HR-ESI-MS m/z calcd for $\mathrm{C}_{18} \mathrm{H}_{20} \mathrm{O}[\mathrm{M}+\mathrm{H}]^{+}:$253.1587, found: 253.1591 .

Method C:
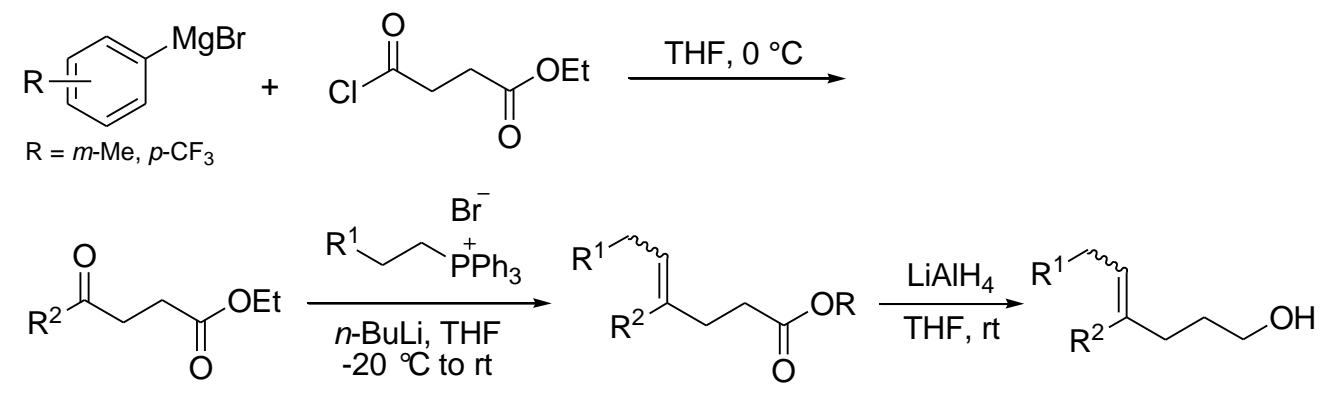

General procedure $\mathrm{C}$ for the synthesis of substrates $\mathbf{1 i}$ and $\mathbf{1 k}$.

To an oven-dried $50 \mathrm{~mL}$ two-neck Schlenk flask equipped with a stir bar and Graham condenser was added magnesium turnings $(420 \mathrm{mg}, 17.3 \mathrm{mmol})$. The flask was then evacuated and back-filled with nitrogen. Next, a solution of bromide ( $3 \mathrm{mmol})$ in anhydrous THF (1.0 M solution) was added via syringe to initiate the reaction and the heterogeneous mixture was stirred at $40{ }^{\circ} \mathrm{C}$. While the colorless solution turned gray, another portion of bromide (13.5 mmol) in anhydrous THF (1.0 M solution) was added dropwise via syringe to maintain a gentle reflux. After stirred at reflux for additional $2 \mathrm{~h}$, the resulting Grignard reagent was cooled down and used for the next step without purification.

An oven-dried $50 \mathrm{~mL}$ Schlenk flask with a stir bar was evacuated and back-filled with nitrogen. Then, ethyl succinyl chloride $(2.14 \mathrm{~mL}, 15 \mathrm{mmol})$ and anhydrous THF (20 
$\mathrm{mL}$ ) was added via syringe. The colorless solution was cooled to $0{ }^{\circ} \mathrm{C}$ and added a solution of Grignard reagent in THF (prepared by the above procedure) via syringe over $20 \mathrm{~min}$. After stirred for additional $6 \mathrm{~h}$ at $0{ }^{\circ} \mathrm{C}$, the resulting mixture was quenched with saturated aqueous $\mathrm{NH}_{4} \mathrm{Cl}$ solution $(60 \mathrm{~mL})$ and extracted with EtOAc $(60 \mathrm{~mL} \times 3)$. The organic extracts were dried over anhydrous $\mathrm{Na}_{2} \mathrm{SO}_{4}$, filtered and concentrated in vacuo. The residue was purified by silica gel column chromatography to afford the keto ester.

The synthesis of olefinic ester and olefinic alcohol is similar to the general procedure A.<smiles>CCCC=C(CCCO)c1cccc(C)c1</smiles>

4-(M-tolyl)oct-4-en-1-ol (1i) $(E / Z=3: 1)$ : Prepared according to general procedure $\mathrm{C}$. Step 1: Flash column chromatography (eluent: PE/EtOAc $=15: 1, \mathrm{v} / \mathrm{v}$ ) to afford keto ester $(2.34 \mathrm{~g}, 71 \%)$ as a yellow oil. Step 2: Flash column chromatography (eluent: $\mathrm{PE} / \mathrm{EtOAc}=60: 1, \mathrm{v} / \mathrm{v})$ to afford olefinic ester $(437 \mathrm{mg}, 17 \%)$ as an amber oil. Step 3: Flash column chromatography (eluent: PE/EtOAc $=6: 1, \mathrm{v} / \mathrm{v}$ ) to afford $1 \mathbf{i}(406 \mathrm{mg}$, 93\%) as a colorless oil. E-isomer, ${ }^{1} \mathrm{H}$ NMR $\left(400 \mathrm{MHz}, \mathrm{CDCl}_{3}\right) \delta 7.21(\mathrm{t}, J=7.4 \mathrm{~Hz}$, $1 \mathrm{H}), 7.15(\mathrm{t}, J=9.3 \mathrm{~Hz}, 1 \mathrm{H}), 7.05(\mathrm{~d}, J=7.4 \mathrm{~Hz}, 1 \mathrm{H}), 6.97-6.93(\mathrm{~m}, 1 \mathrm{H}), 5.46(\mathrm{t}, J$ $=7.3 \mathrm{~Hz}, 1 \mathrm{H}), 3.62(\mathrm{t}, J=6.4 \mathrm{~Hz}, 2 \mathrm{H}), 2.41(\mathrm{t}, J=7.4 \mathrm{~Hz}, 2 \mathrm{H}), 2.35(\mathrm{~s}, 3 \mathrm{H}), 1.91(\mathrm{q}$, $J=7.3 \mathrm{~Hz}, 2 \mathrm{H}), 1.61-1.54(\mathrm{~m}, 2 \mathrm{H}), 1.39-1.31(\mathrm{~m}, 2 \mathrm{H}), 0.84(\mathrm{t}, J=7.4 \mathrm{~Hz}, 3 \mathrm{H})$. ${ }^{13} \mathrm{C}$ NMR $\left(101 \mathrm{MHz}, \mathrm{CDCl}_{3}\right) \delta 141.1,140.5,137.6,129.2,128.0,127.8,127.3,125.7$, 62.7, 35.7, 31.3, 31.1, 23.4, 21.6, 13.9. HR-ESI-MS m/z calcd for $\mathrm{C}_{15} \mathrm{H}_{22} \mathrm{O}[\mathrm{M}+\mathrm{H}]^{+}$: 219.1743, found: 219.1747 .<smiles>OCCCC(=CCCc1ccccc1)c1ccc(C(F)(F)F)cc1</smiles>

7-Phenyl-4-(4-(trifluoromethyl)phenyl)hept-4-en-1-ol $(1 \mathbf{k})(E / Z=2: 1)$ : Prepared according to general procedure C. Step 1: Flash column chromatography (eluent: $\mathrm{PE} / \mathrm{EtOAc}=15: 1, \mathrm{v} / \mathrm{v})$ to afford keto ester $(2.13 \mathrm{~g}, 52 \%)$ as a pale yellow solid. Step 2: Flash column chromatography (eluent: PE/EtOAc $=40: 1, \mathrm{v} / \mathrm{v}$ ) to afford olefinic ester (471 mg, 13\%) as a yellow oil. Step 3: Flash column chromatography (eluent: $\mathrm{PE} / \mathrm{EtOAc}=6: 1, \mathrm{v} / \mathrm{v})$ to afford $\mathbf{1 k}(624 \mathrm{mg}, 93 \%)$ as a pale yellow oil. $E$-isomer, ${ }^{1} \mathrm{H}$ NMR (400 MHz, $\left.\mathrm{CDCl}_{3}\right) \delta 7.55(\mathrm{~d}, J=7.9 \mathrm{~Hz}, 2 \mathrm{H}), 7.33-7.16(\mathrm{~m}, 5 \mathrm{H}), 7.06(\mathrm{~d}, J=$ $7.9 \mathrm{~Hz}, 2 \mathrm{H}), 5.57(\mathrm{t}, J=7.4 \mathrm{~Hz}, 1 \mathrm{H}), 3.58(\mathrm{t}, J=6.4 \mathrm{~Hz}, 2 \mathrm{H}), 2.64(\mathrm{t}, J=7.5 \mathrm{~Hz}, 2 \mathrm{H})$, $2.41(\mathrm{t}, J=7.5 \mathrm{~Hz}, 2 \mathrm{H}), 2.22(\mathrm{q}, J=7.4 \mathrm{~Hz}, 2 \mathrm{H}), 1.57-1.42(\mathrm{~m}, 2 \mathrm{H}) .{ }^{13} \mathrm{C}$ NMR $(101$ $\left.\mathrm{MHz}, \mathrm{CDCl}_{3}\right) \delta 144.77,141.64,140.11,130.54,129.17(\mathrm{~d}, J=32.2 \mathrm{~Hz}), 128.68$, $128.40,126.73,126.02,125.16(\mathrm{q}, J=3.8 \mathrm{~Hz}), 124.38(\mathrm{~d}, J=272.1 \mathrm{~Hz}), 62.40,36.17$, 35.38, 31.03, 30.85. HR-ESI-MS m/z calcd for $\mathrm{C}_{20} \mathrm{H}_{21} \mathrm{~F}_{3} \mathrm{O}[\mathrm{M}-\mathrm{H}]^{-}: 333.1472$, found: 333.1470 . 
Method D:

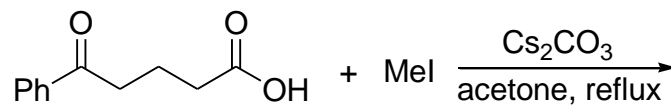<smiles>COC(=O)CCCC(=O)c1ccccc1</smiles>
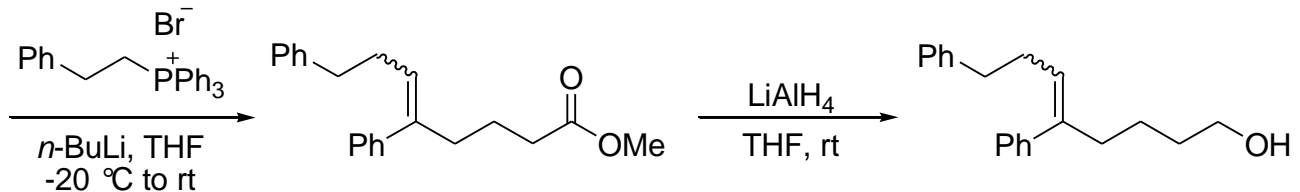

Procedure D for the synthesis of substrate $1 \mathbf{r}$.

A $50 \mathrm{~mL}$ round-bottom flask was equipped with a stir bar and Graham condenser, and charged with 4-benzoylbutyric acid (2.11 g, $11 \mathrm{mmol}), \mathrm{Cs}_{2} \mathrm{CO}_{3}(1.79 \mathrm{~g}, 5.5 \mathrm{mmol})$ and acetone $(18 \mathrm{~mL})$. Then, iodomethane $(1.37 \mathrm{~mL}, 22 \mathrm{mmol})$ was added via syringe and the heterogeneous mixture was stirred at reflux for $17 \mathrm{~h}$. The resulting mixture was diluted with $\mathrm{Et}_{2} \mathrm{O}(40 \mathrm{~mL})$ and washed with brine. The organic phase was dried over anhydrous $\mathrm{Na}_{2} \mathrm{SO}_{4}$, filtered and concentrated in vacuo. The residue was purified by silica gel column chromatography to afford the keto ester.

The synthesis of olefinic ester and olefinic alcohol is similar to the general procedure A.<smiles>OCCCCC(=CCCc1ccccc1)c1ccccc1</smiles>

5,8-Diphenyloct-5-en-1-ol (1r) $(E / Z=5: 1)$ : Prepared according to procedure D. Step 1: Flash column chromatography (eluent: $\mathrm{PE} / \mathrm{EtOAc}=15: 1 \mathrm{v} / \mathrm{v}$ ) to afford keto ester $(1.92 \mathrm{~g}, 85 \%)$ as a pale yellow oil. Step 2: Flash column chromatography (eluent: $\mathrm{PE} / \mathrm{EtOAc}=50: 1, \mathrm{v} / \mathrm{v})$ to afford olefinic ester $(1.70 \mathrm{~g}, 55 \%)$ as a pale yellow oil. Step 3: Flash column chromatography (eluent: $\mathrm{PE} / \mathrm{EtOAc}=6: 1, \mathrm{v} / \mathrm{v})$ to afford $\mathbf{1 r}(527 \mathrm{mg}$, $94 \%)$ as a colorless oil. $E$-isomer, ${ }^{1} \mathrm{H}$ NMR $\left(400 \mathrm{MHz}, \mathrm{CDCl}_{3}\right) \delta 7.33-7.27(\mathrm{~m}, 3 \mathrm{H})$, $7.26-7.15(\mathrm{~m}, 3 \mathrm{H}), 7.10(\mathrm{~d}, J=6.9 \mathrm{~Hz}, 2 \mathrm{H}), 7.03(\mathrm{~d}, J=8.3 \mathrm{~Hz}, 2 \mathrm{H}), 5.48(\mathrm{t}, J=7.3$ $\mathrm{Hz}, 1 \mathrm{H}), 3.58(\mathrm{t}, J=6.6 \mathrm{~Hz}, 2 \mathrm{H}), 2.64(\mathrm{t}, J=7.7 \mathrm{~Hz}, 2 \mathrm{H}), 2.35(\mathrm{t}, J=7.2 \mathrm{~Hz}, 2 \mathrm{H})$, $2.25(\mathrm{dd}, J=15.2,7.5 \mathrm{~Hz}, 2 \mathrm{H}), 1.58-1.46(\mathrm{~m}, 2 \mathrm{H}), 1.41-1.28(\mathrm{~m}, 2 \mathrm{H}) .{ }^{13} \mathrm{C} \mathrm{NMR}$ $\left(101 \mathrm{MHz}, \mathrm{CDCl}_{3}\right) \delta 142.1,141.6,141.1,128.7,128.4,128.3,128.1,126.6,126.6$, $125.8,63.0,39.1,36.5,32.3,30.9,24.3$. HR-ESI-MS m/z calcd for $\mathrm{C}_{20} \mathrm{H}_{24} \mathrm{O}[\mathrm{M}+\mathrm{H}]^{+}$: 281.1900, found: 281.1905 .

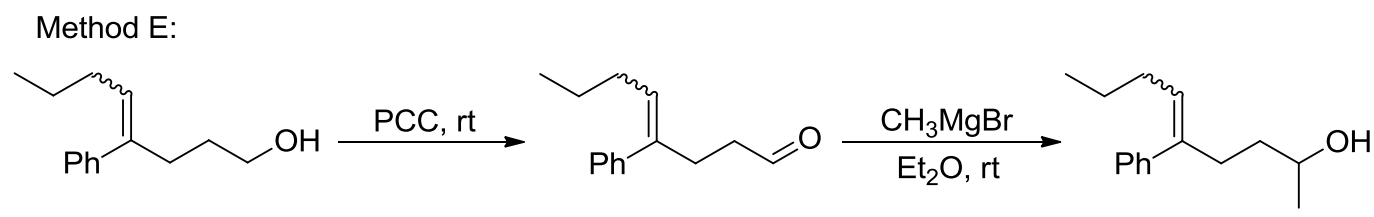

Procedure E for the synthesis of substrate 1p

An oven-dried $50 \mathrm{~mL}$ Schlenk flask equipped with a stir bar was charged with PCC (1.08 g, $5 \mathrm{mmol})$. The flask was then evacuated and back-filled with nitrogen. Then anhydrous DCM (12 mL) was added via syringe. To the colorless solution was added 
olefinic alcohol (511 mg, $2.5 \mathrm{mmol})$ in anhydrous DCM (6 mL) via syringe at room temperature. After stirred for $4 \mathrm{~h}$, the resulting heterogeneous mixture was diluted with $\mathrm{Et}_{2} \mathrm{O}(10 \mathrm{~mL})$ and filtered through Florisil. Then the Florisil was washed with ether $(50 \mathrm{~mL})$ and the solution was concentrated in vacuo. The residue was purified by silica gel column chromatography to afford the olefinic aldehyde.

An oven-dried $25 \mathrm{~mL}$ Schlenk flask with a stir bar was evacuated and back-filled with nitrogen. Then, olefinic aldehyde (445 mg, $2.2 \mathrm{mmol})$ and anhydrous $\mathrm{Et}_{2} \mathrm{O}(8 \mathrm{~mL})$ was added via syringe and cooled to $0{ }^{\circ} \mathrm{C}$. To the colorless solution was added

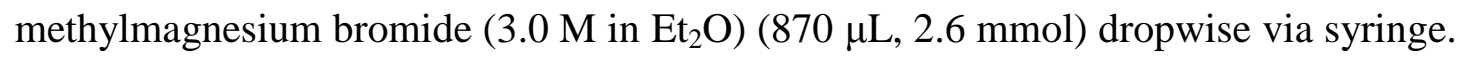
The resulting solution was warmed to room temperature gradually and stirred overnight, after which the homogeneous mixture was quenched with saturated aqueous $\mathrm{NH}_{4} \mathrm{Cl}$ solution $(30 \mathrm{~mL})$ and extracted with $\mathrm{DCM}(30 \mathrm{~mL} \times 3)$. The organic extracts were dried over anhydrous $\mathrm{Na}_{2} \mathrm{SO}_{4}$, filtered and concentrated in vacuo. The residue was purified by silica gel column chromatography to afford the olefinic ester.<smiles>CCCC=C(CCC(C)O)c1ccccc1</smiles>

5-Phenylnon-5-en-2-ol (1p) $(E / Z=4: 1)$ : Prepared according to procedure E. Step 1: Flash column chromatography (eluent: PE/EtOAc $=20: 1, \mathrm{v} / \mathrm{v}$ ) to afford olefinic aldehyde (439 $\mathrm{mg}, 87 \%$ ) as a pale yellow oil. Step 2: Flash column chromatography (eluent: $\mathrm{PE} / \mathrm{EtOAc}=6: 1, \mathrm{v} / \mathrm{v})$ to afford $1 p(436 \mathrm{mg}, 91 \%)$ as a colorless oil. $E$-isomer, ${ }^{1} \mathrm{H}$ NMR (400 MHz, $\left.\mathrm{CDCl}_{3}\right) \delta 7.34-7.29(\mathrm{~m}, 2 \mathrm{H}), 7.26-7.21(\mathrm{~m}, 1 \mathrm{H}), 7.14(\mathrm{~d}, J=$ $6.9 \mathrm{~Hz}, 2 \mathrm{H}), 5.49(\mathrm{t}, J=7.3 \mathrm{~Hz}, 1 \mathrm{H}), 3.84-3.73(\mathrm{~m}, 1 \mathrm{H}), 2.52-2.33(\mathrm{~m}, 2 \mathrm{H}), 1.91$ $(\mathrm{q}, J=7.3 \mathrm{~Hz}, 2 \mathrm{H}), 1.52-1.41(\mathrm{~m}, 2 \mathrm{H}), 1.33(\mathrm{dt}, J=14.6,7.3 \mathrm{~Hz}, 2 \mathrm{H}), 1.15(\mathrm{~d}, J=$ $6.2 \mathrm{~Hz}, 3 \mathrm{H}), 0.84(\mathrm{t}, J=7.4 \mathrm{~Hz}, 3 \mathrm{H}) .{ }^{13} \mathrm{C} \mathrm{NMR}\left(101 \mathrm{MHz}, \mathrm{CDCl}_{3}\right) \delta 141.3,140.7$, 128.6, 128.2, 127.8, 126.5, 68.0, 37.8, 35.8, 31.1, 23.6, 23.3, 13.9. HR-ESI-MS m/z calcd for $\mathrm{C}_{15} \mathrm{H}_{22} \mathrm{O}[\mathrm{M}+\mathrm{H}]^{+}: 219.1743$, found: 219.1744 .

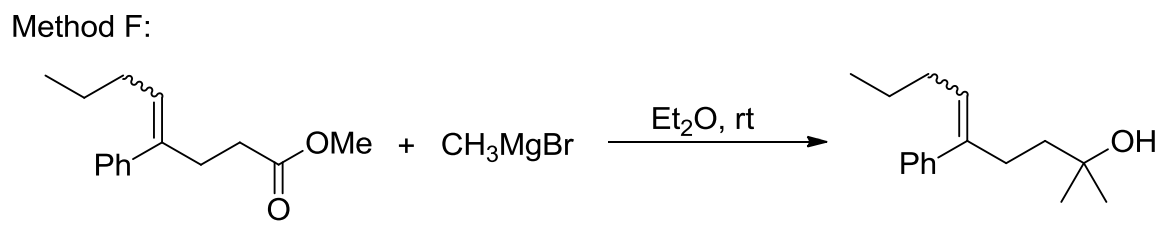

Procedure $\mathrm{F}$ for the synthesis of substrate $\mathbf{1 q}$

An oven-dried $25 \mathrm{~mL}$ Schlenk flask with a stir bar was evacuated and back-filled with nitrogen. Then, olefinic ester $(558 \mathrm{mg}, 2.4 \mathrm{mmol})$ and anhydrous $\mathrm{Et}_{2} \mathrm{O}(10 \mathrm{~mL})$ was added via syringe. To the colorless solution was added methylmagnesium bromide $\left(3.0 \mathrm{M}\right.$ in $\left.\mathrm{Et}_{2} \mathrm{O}\right)(2 \mathrm{~mL}, 6 \mathrm{mmol})$ dropwise via syringe. After stirred at room temperature for $2.5 \mathrm{~h}$, the resulting mixture was quenched with saturated aqueous $\mathrm{NH}_{4} \mathrm{Cl}$ solution $(40 \mathrm{~mL})$ and extracted with $\mathrm{DCM}(40 \mathrm{~mL} \times 3)$. The organic extracts were dried over anhydrous $\mathrm{Na}_{2} \mathrm{SO}_{4}$, filtered and concentrated in vacuo. The residue was purified by silica gel column chromatography to afford the olefinic alcohol. 
<smiles>CCCC=C(CCC(C)(C)O)c1ccccc1</smiles>

2-Methyl-5-phenylnon-5-en-2-ol (1q) $(E / Z=3: 1)$ : Prepared according to procedure F. Flash column chromatography (eluent: $\mathrm{PE} / \mathrm{EtOAc}=6: 1, \mathrm{v} / \mathrm{v})$ to afford $1 \mathrm{q}(506 \mathrm{mg}$, 91\%) as a colorless oil. E-isomer, ${ }^{1} \mathrm{H}$ NMR (400 $\left.\mathrm{MHz} \mathrm{CDCl}_{3}\right) \delta 7.36-7.29(\mathrm{~m}, 2 \mathrm{H})$, $7.24(\mathrm{t}, J=7.4 \mathrm{~Hz}, 1 \mathrm{H}), 7.14(\mathrm{~d}, J=6.8 \mathrm{~Hz}, 2 \mathrm{H}), 5.49(\mathrm{t}, J=7.3 \mathrm{~Hz}, 1 \mathrm{H}), 2.46-2.38$ $(\mathrm{m}, 2 \mathrm{H}), 1.90(\mathrm{q}, J=7.4 \mathrm{~Hz}, 2 \mathrm{H}), 1.54-1.46(\mathrm{~m}, 2 \mathrm{H}), 1.39-1.29(\mathrm{~m}, 2 \mathrm{H}), 1.18$ (s, $6 \mathrm{H}), 0.84(\mathrm{t}, J=7.4 \mathrm{~Hz}, 3 \mathrm{H}) .{ }^{13} \mathrm{C}$ NMR $\left(101 \mathrm{MHz}, \mathrm{CDCl}_{3}\right) \delta 141.4,141.1,128.6$, 128.1, 127.4, 126.5, 71.1, 42.6, 34.3, 31.1, 29.3, 23.4, 13.9. HR-ESI-MS m/z calcd for $\mathrm{C}_{16} \mathrm{H}_{24} \mathrm{O}[\mathrm{M}+\mathrm{H}]^{+}: 233.1900$, found: 233.1898 .

Method G:
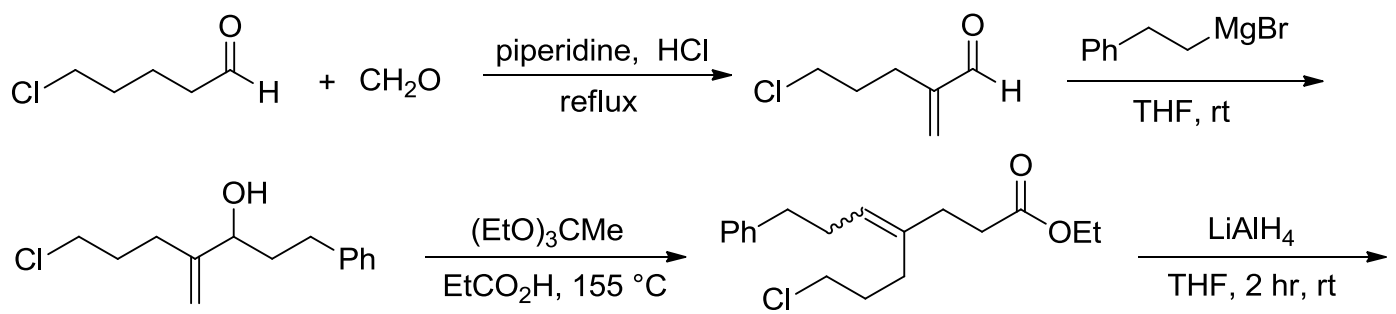<smiles>OCCCC(=CCCc1ccccc1)CCCCl</smiles>

Procedure $\mathrm{G}$ for the synthesis of substrate 1e. This is a modified literature procedure. ${ }^{3}$ An oven-dried $4 \mathrm{~mL}$ vial with a stir bar was charged with 5-chloropentanal ( $836 \mu \mathrm{L}, 7$ mmol), formaldehyde (36\% 38\% aqueous solution, $668 \mu \mathrm{L}, 8.4 \mathrm{mmol}$ ), piperidine $(64.1 \mu \mathrm{L}, 0.7 \mathrm{mmol})$ and $6 \mathrm{M} \mathrm{HCl}(58.3 \mu \mathrm{L}, 0.35 \mathrm{mmol})$ as follow. The heterogeneous mixture was stirred vigorously at reflux for $12 \mathrm{~h}$. The resulting solution was cooled down, acidified by aqueous $1 \mathrm{M} \mathrm{HCl}$ solution $(800 \mu \mathrm{L})$, and stirred for additional $1 \mathrm{~h}$. And then the mixture was diluted with $\mathrm{H}_{2} \mathrm{O}(10 \mathrm{~mL})$ and extracted with DCM $(10 \mathrm{~mL}$ $\times 3)$. The organic extracts were dried over anhydrous $\mathrm{Na}_{2} \mathrm{SO}_{4}$, filtered and concentrated in vacuo. The crude $\alpha, \beta$-unsaturated aldehyde was used directly to the next step without further purification.

An oven-dried $50 \mathrm{~mL}$ Schlenk flask equipped with a stir bar was evacuated and back-filled with nitrogen. Then, $\alpha, \beta$-unsaturated aldehyde $(928 \mathrm{mg}, 7 \mathrm{mmol})$ and anhydrous THF $(15 \mathrm{~mL})$ was added via syringe. To the colorless solution was added phenethyl- magnesium bromide (prepared by procedure C) (1.0 M in THF) (14 mL, $14 \mathrm{mmol}$ ) dropwise over $15 \mathrm{~min}$. After stirred at room temperature for $2 \mathrm{~h}$, the resulting mixture was quenched with saturated aqueous $\mathrm{NH}_{4} \mathrm{Cl}$ solution $(50 \mathrm{~mL})$ and extracted with DCM $(50 \mathrm{~mL} \times 3)$. The organic extracts were dried over anhydrous $\mathrm{Na}_{2} \mathrm{SO}_{4}$, filtered and concentrated in vacuo. The residue was purified by silica gel column chromatography to afford the allylic alcohol.

An oven-dried $15 \mathrm{~mL}$ sealed tube with a stir bar was charged with allylic alcohol 
$(1.64 \mathrm{~g}, 6.85 \mathrm{mmol})$, triethyl orthoacetate $(7.18 \mathrm{~mL}, 39.4 \mathrm{mmol})$, and propionic acid $(25.6 \mu \mathrm{L}, 0.34 \mathrm{mmol})$ as follow. The homogeneous mixture was stirred at $155^{\circ} \mathrm{C}$ for 2 $\mathrm{h}$, after which the resulting mixture was cooled down, treated with aqueous $3 \mathrm{M}$ $\mathrm{H}_{2} \mathrm{SO}_{4}$ solution $(6 \mathrm{~mL})$, and stirred for additional $20 \mathrm{~min}$ at room temperature. Then the mixture was diluted with $\mathrm{H}_{2} \mathrm{O}(50 \mathrm{~mL})$ and extracted with $\mathrm{DCM}(50 \mathrm{~mL} \times 3)$. The organic extracts were dried over anhydrous $\mathrm{Na}_{2} \mathrm{SO}_{4}$, filtered and concentrated in vacuo. The residue was purified by silica gel column chromatography to afford the olefinic ester.

The synthesis of olefinic alcohol is similar to the general procedure A.<smiles>OCCCC(=CCCc1ccccc1)CCCCl</smiles>

5-(3-Chloropropyl)-8-phenyloct-5-en-1-ol (1e) $(Z / E=11: 1)$ : Prepared according to procedure G. Step 1 and Step 2: Flash column chromatography (eluent: PE/EtOAc = $8: 1, \mathrm{v} / \mathrm{v})$ to afford allylic alcohol $(819 \mathrm{mg}, 49 \%)$ as a pale yellow oil. Step 3: Flash column chromatography (eluent: PE/EtOAc $=40: 1, \mathrm{v} / \mathrm{v})$ to afford olefinic ester $(1.18$ $\mathrm{g}, 56 \%$ ) as a pale yellow oil. Step 4: Flash column chromatography (eluent: $\mathrm{PE} / \mathrm{EtOAc}=6: 1, \mathrm{v} / \mathrm{v})$ to afford $1 \mathrm{e}(234 \mathrm{mg}, 44 \%)$ as a colorless oil. $E$-isomer, ${ }^{1} \mathrm{H}$ NMR (400 MHz, $\left.\mathrm{CDCl}_{3}\right) \delta 7.19(\mathrm{~d}, J=7.7 \mathrm{~Hz}, 2 \mathrm{H}), 7.09(\mathrm{t}, J=5.9 \mathrm{~Hz}, 3 \mathrm{H}), 5.17(\mathrm{t}, J$ $=7.2 \mathrm{~Hz}, 1 \mathrm{H}), 3.55-3.47(\mathrm{~m}, 2 \mathrm{H}), 3.36(\mathrm{t}, J=6.5 \mathrm{~Hz}, 2 \mathrm{H}), 2.55(\mathrm{t}, J=7.7 \mathrm{~Hz}, 2 \mathrm{H})$, $2.25(\mathrm{dd}, J=15.0,7.4 \mathrm{~Hz}, 2 \mathrm{H}), 2.06-1.99(\mathrm{~m}, 2 \mathrm{H}), 1.95(\mathrm{t}, J=7.6 \mathrm{~Hz}, 2 \mathrm{H}), 1.70-$ $1.60(\mathrm{~m}, 2 \mathrm{H}), 1.59-1.50(\mathrm{~m}, 2 \mathrm{H}) .{ }^{13} \mathrm{C}$ NMR $\left(101 \mathrm{MHz}, \mathrm{CDCl}_{3}\right) \delta 142.1,137.8,128.6$, 128.4, 125.9, 125.9, 62.7, 44.9, 36.4, 33.0, 31.2, 31.1, 29.9, 27.2. HR-ESI-MS m/z calcd for $\mathrm{C}_{16} \mathrm{H}_{23} \mathrm{ClO}[\mathrm{M}+\mathrm{Na}]^{+}$: 289.1330, found: 261.1327 .

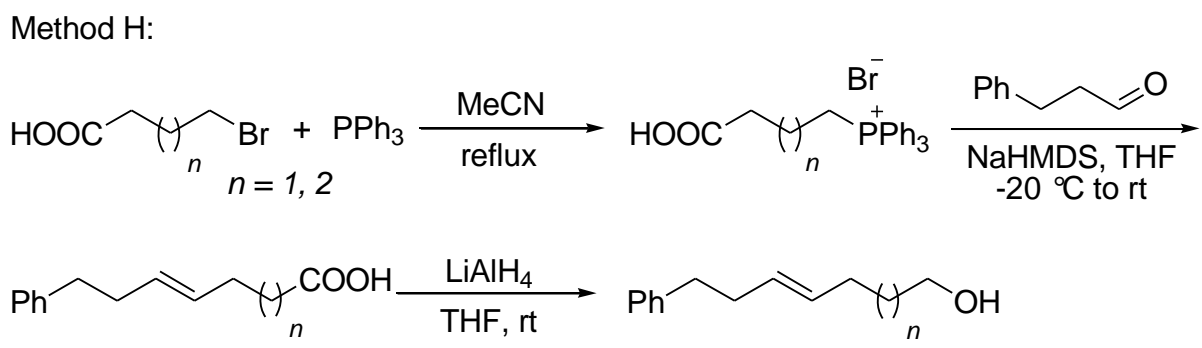

General procedure $\mathrm{H}$ for the synthesis of substrates $\mathbf{1 g}$ and $1 \mathrm{~s}$. This is a modified literature procedure. ${ }^{4}$

To an oven-dried $50 \mathrm{~mL}$ Schlenk flask equipped with a stir bar and Graham condenser, was added $\mathrm{PPh}_{3}(2.62 \mathrm{~g}, 10 \mathrm{mmol})$. The flask was then evacuated and back-filled with nitrogen. Next, bromide $(10 \mathrm{mmol})$ and $10 \mathrm{~mL}$ anhydrous $\mathrm{MeCN}$ was added via syringe. The homogeneous mixture was stirred at reflux for $36 \mathrm{~h}$. The resulting colorless solution was cooled to room temperature and then $30 \mathrm{~mL} \mathrm{Et}_{2} \mathrm{O}$ was added. After keeping at $-20{ }^{\circ} \mathrm{C}$ for $4 \mathrm{~h}$, the formed precipitate was filtered, washed with $\mathrm{Et}_{2} \mathrm{O}$ $(20 \mathrm{~mL} \times 3)$, and dried in vacuo to afford the triphenylphosphonium bromide as a white solid. 
An oven-dried $50 \mathrm{~mL}$ Schlenk flask equipped with a stir bar was charged with triphenylphosphonium bromide $(10 \mathrm{mmol})$. The flask was then evacuated and back-filled with nitrogen. Then $20 \mathrm{~mL}$ anhydrous THF was added via syringe and the suspension was cooled to $-20^{\circ} \mathrm{C}$. To the colorless suspension was added NaHMDS (2.5 $\mathrm{M}$ in THF) $(8.8 \mathrm{~mL}, 22 \mathrm{mmol})$ dropwise via syringe over $5 \mathrm{~min}$. The resulting orange solution was stirred at $-20^{\circ} \mathrm{C}$ for $30 \mathrm{~min}$ and became homogeneous gradually. Then a solution of phenylpropyl aldehyde $(1.19 \mathrm{~mL}, 9 \mathrm{mmol})$ in $2 \mathrm{~mL}$ anhydrous THF was added via syringe at $-20^{\circ} \mathrm{C}$. The resulting mixture was allowed to warm to room temperature and stirred overnight, after which the heterogeneous mixture was quenched with $\mathrm{H}_{2} \mathrm{O}(50 \mathrm{~mL})$ and washed with $\mathrm{DCM}(50 \mathrm{~mL} \times 2)$. The aqueous phase was acidified to $\mathrm{pH}=1$ by aqueous $1 \mathrm{M} \mathrm{HCl}$ solution, and then extracted with EtOAc $(60 \mathrm{~mL} \times 3)$. The organic extracts were dried over anhydrous $\mathrm{Na}_{2} \mathrm{SO}_{4}$, filtered and concentrated in vacuo. The residue was purified by silica gel column chromatography to afford the olefinic ester.

The reduction of olefinic acid is similar to the general procedure A.

$\mathrm{Ph} \sim \mathrm{OH}$

7-Phenylhept-4-en-1-ol (1g) $(E / Z=15: 1)$ : Prepared according to general procedure H. Step 1: Flash column chromatography (eluent: PE/EtOAc $=3: 1, \mathrm{v} / \mathrm{v}$ ) to afford olefinic acid $(547 \mathrm{mg}, 27 \%)$ as a pale yellow oil. Step 2: Flash column chromatography (eluent: $\mathrm{PE} / \mathrm{EtOAc}=6: 1, \mathrm{v} / \mathrm{v})$ to afford $1 \mathrm{~g}(345 \mathrm{mg}, 91 \%)$ as a colorless oil. The ${ }^{1} \mathrm{H}$ NMR spectrum was in agreement with those reported in reference ${ }^{5} . E$-isomer, ${ }^{1} \mathrm{H}$ NMR $\left(400 \mathrm{MHz}, \mathrm{CDCl}_{3}\right) \delta 7.29(\mathrm{~d}, J=7.4 \mathrm{~Hz}, 2 \mathrm{H}), 7.19$ (d, $J=6.9 \mathrm{~Hz}, 3 \mathrm{H}), 5.49-5.35(\mathrm{~m}, 2 \mathrm{H}), 3.57(\mathrm{t}, J=6.5 \mathrm{~Hz}, 2 \mathrm{H}), 2.67(\mathrm{t}, J=7.6 \mathrm{~Hz}, 2 \mathrm{H})$, $2.37(\mathrm{dd}, J=15.0,7.8 \mathrm{~Hz}, 2 \mathrm{H}), 2.06(\mathrm{dd}, J=14.2,7.1 \mathrm{~Hz}, 2 \mathrm{H}), 1.54(\mathrm{dt}, J=13.7,6.6$ $\mathrm{Hz}, 2 \mathrm{H})$.<smiles>OCCCCC=CCCc1ccccc1</smiles>

8-Phenyloct-5-en-1-ol (1s) $(E / Z=6: 1)$ : Prepared according to general procedure $\mathrm{H}$. Step 1: Flash column chromatography (eluent: PE/EtOAc $=3: 1, \mathrm{v} / \mathrm{v}$ ) to afford olefinic acid $(1.53 \mathrm{~g}, 70 \%)$ as a colorless oil. Step 2: Flash column chromatography (eluent: $\mathrm{PE} / \mathrm{EtOAc}=6: 1, \mathrm{v} / \mathrm{v})$ to afford $\mathbf{1 s}(364 \mathrm{mg}, 89 \%)$ as a colorless oil. $E$-isomer, ${ }^{1} \mathrm{H}$ NMR (400 MHz, $\left.\mathrm{CDCl}_{3}\right) \delta 7.29(\mathrm{~d}, J=8.3 \mathrm{~Hz}, 2 \mathrm{H}), 7.20(\mathrm{~d}, J=7.2 \mathrm{~Hz}, 3 \mathrm{H}), 5.47-$ $5.34(\mathrm{~m}, 2 \mathrm{H}), 3.61(\mathrm{t}, J=6.5 \mathrm{~Hz}, 2 \mathrm{H}), 2.67(\mathrm{t}, J=7.7 \mathrm{~Hz}, 2 \mathrm{H}), 2.36(\mathrm{dd}, J=14.7,7.9$ $\mathrm{Hz}, 2 \mathrm{H}), 2.01(\mathrm{dd}, J=14.2,7.1 \mathrm{~Hz}, 2 \mathrm{H}), 1.57-1.48(\mathrm{~m}, 2 \mathrm{H}), 1.41-1.27(\mathrm{~m}, 2 \mathrm{H})$.

${ }^{13} \mathrm{C} \mathrm{NMR}\left(101 \mathrm{MHz}, \mathrm{CDCl}_{3}\right) \delta 142.2,130.3,129.2,128.6,128.4,125.9,63.0,36.1$, 32.4, 29.4, 27.0, 25.8. HR-ESI-MS m/z calcd for $\mathrm{C}_{14} \mathrm{H}_{20} \mathrm{O}[\mathrm{M}+\mathrm{H}]^{+}:$205.1587, found: 205.1585 . 


\subsection{Synthesis of olefinic sulfonamides}

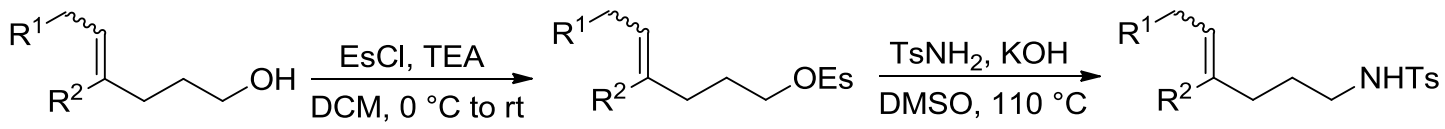

General procedure for the synthesis of substrates $\mathbf{4 a - 4 f}$. This is a modified literature procedure. ${ }^{6}$

An oven-dried $25 \mathrm{~mL}$ Schlenk flask with a stir bar was evacuated and back-filled with nitrogen. Then, olefinic alcohol $(1.2 \mathrm{mmol})$, TEA $(347 \mu \mathrm{L}, 2.5 \mathrm{mmol})$ and anhydrous DCM $(8 \mathrm{~mL})$ was added. Then the colorless solution was cooled to $0{ }^{\circ} \mathrm{C}$ and ethanesulfonyl chloride $(152 \mu \mathrm{L}, 1.6 \mathrm{mmol})$ was added in one portion. The resulting mixture was warmed to room temperature gradually and stirred for $4 \mathrm{~h}$, after which the brown solution was quenched with $\mathrm{H}_{2} \mathrm{O}(20 \mathrm{~mL})$ and extracted with DCM $(20 \mathrm{~mL}$ $\times 3$ ). The organic extracts were dried over anhydrous $\mathrm{Na}_{2} \mathrm{SO}_{4}$, filtered and concentrated in vacuo. The crude sulfonate was used for the next step without further purification.

A $50 \mathrm{~mL}$ round-bottom flask was equipped with a stir bar and Graham condenser, and charged with $\mathrm{KOH}(101 \mathrm{mg}, 1.8 \mathrm{mmol}), \mathrm{TsNH}_{2}(308 \mathrm{mg}, 1.8 \mathrm{mmol})$ and $10 \mathrm{~mL}$ DMSO. Then, the heterogeneous mixture was stirred at $110^{\circ} \mathrm{C}$ for $30 \mathrm{~min}$ and became homogeneous gradually. Then a solution of sulfonate $(1.2 \mathrm{mmol})$ in DMSO $(3 \mathrm{~mL})$ was added dropwise via syringe at $110^{\circ} \mathrm{C}$ over $5 \mathrm{~min}$. After stirred at $110^{\circ} \mathrm{C}$ for $2 \mathrm{~h}$, the resulting mixture was cooled down, diluted with $\mathrm{H}_{2} \mathrm{O}(10 \mathrm{~mL})$ and extracted with $\mathrm{Et}_{2} \mathrm{O}(20 \mathrm{~mL} \times 3)$. The organic extracts were dried over anhydrous $\mathrm{Na}_{2} \mathrm{SO}_{4}$, filtered and concentrated in vacuo. The residue was purified by silica gel column chromatography to afford the olefinic sulfonamide.<smiles>CCCCC(=CCCc1ccccc1)c1ccccc1</smiles>

$N$-(4,7-Diphenylhept-4-en-1-yl)-4-methylbenzenesulfonamide $(4 \mathbf{a}) \quad(E / Z=4: 1)$ : Prepared according to general procedure. Step 1 and Step 2: Flash column chromatography (eluent: $\mathrm{PE} / \mathrm{EtOAc}=20: 1$ to $\mathrm{PE} / \mathrm{EtOAc}=6: 1, \mathrm{v} / \mathrm{v})$ to afford $\mathbf{4 a}(322$ $\mathrm{mg}, 64 \%)$ as a colorless oil. E-isomer, ${ }^{1} \mathrm{H} \mathrm{NMR}\left(400 \mathrm{MHz}, \mathrm{CDCl}_{3}\right) \delta 7.72(\mathrm{~d}, J=8.2$ $\mathrm{Hz}, 2 \mathrm{H}), 7.33-7.20(\mathrm{~m}, 8 \mathrm{H}), 7.07(\mathrm{~d}, J=7.2 \mathrm{~Hz}, 2 \mathrm{H}), 6.92(\mathrm{~d}, J=6.8 \mathrm{~Hz}, 2 \mathrm{H}), 5.42$ $(\mathrm{t}, J=7.3 \mathrm{~Hz}, 1 \mathrm{H}), 4.49$ (t, $J=6.1 \mathrm{~Hz}, 1 \mathrm{H}), 2.88(\mathrm{q}, J=6.8 \mathrm{~Hz}, 2 \mathrm{H}), 2.59$ (t, $J=7.7$ $\mathrm{Hz}, 2 \mathrm{H}), 2.42(\mathrm{~s}, 3 \mathrm{H}), 2.30(\mathrm{t}, J=7.4 \mathrm{~Hz}, 2 \mathrm{H}), 2.20(\mathrm{dd}, J=15.1,7.5 \mathrm{~Hz}, 2 \mathrm{H}), 1.41(\mathrm{p}$, $J=7.1 \mathrm{~Hz}, 2 \mathrm{H}) .{ }^{13} \mathrm{C} \mathrm{NMR}\left(101 \mathrm{MHz}, \mathrm{CDCl}_{3}\right) \delta 143.4,141.9,140.4,140.3,137.1$, 129.8, 128.6, 128.3, 128.2, 128.2, 127.5, 127.2, 126.8, 125.9, 42.7, 36.3, 36.2, 30.8, 27.9, 21.6. HR-ESI-MS $\mathrm{m} / \mathrm{z}$ calcd for $\mathrm{C}_{26} \mathrm{H}_{29} \mathrm{NO}_{2} \mathrm{~S}[\mathrm{M}+\mathrm{H}]^{+}: 420.1992$, found: 420.1992 .<smiles>CCC/C=C(/CCCNC(C)C)c1ccc(C)cc1</smiles> 
4-Methyl- $\boldsymbol{N}$-(4-(p-tolyl)oct-4-en-1-yl)benzenesulfonamide $(\mathbf{4 b}) \quad(E / Z=3: 1)$ : Prepared according to general procedure. Step 1 and Step 2: Flash column chromatography (eluent: PE/EtOAc $=20: 1$ to PE/EtOAc $=7: 1, \mathrm{v} / \mathrm{v})$ to afford $\mathbf{4 b}(304$ $\mathrm{mg}, 80 \%)$ as a colorless oil. $E$-isomer, ${ }^{1} \mathrm{H} \mathrm{NMR}\left(400 \mathrm{MHz}, \mathrm{CDCl}_{3}\right) \delta 7.71(\mathrm{~d}, J=8.2$ $\mathrm{Hz}, 2 \mathrm{H}), 7.28(\mathrm{~d}, J=7.9 \mathrm{~Hz}, 2 \mathrm{H}), 7.10(\mathrm{~d}, J=7.6 \mathrm{~Hz}, 2 \mathrm{H}), 6.93(\mathrm{~d}, J=7.9 \mathrm{~Hz}, 2 \mathrm{H})$, $5.35(\mathrm{t}, J=7.3 \mathrm{~Hz}, 1 \mathrm{H}), 4.43(\mathrm{t}, J=5.7 \mathrm{~Hz}, 1 \mathrm{H}), 2.96-2.83(\mathrm{~m}, 2 \mathrm{H}), 2.43(\mathrm{~s}, 3 \mathrm{H})$, $2.34(\mathrm{~s}, 3 \mathrm{H}), 2.30(\mathrm{t}, J=7.3 \mathrm{~Hz}, 2 \mathrm{H}), 1.86(\mathrm{q}, J=7.3 \mathrm{~Hz}, 2 \mathrm{H}), 1.51-1.38(\mathrm{~m}, 2 \mathrm{H})$, $1.34-1.22(\mathrm{~m}, 2 \mathrm{H}), 0.81(\mathrm{t}, J=7.4 \mathrm{~Hz}, 3 \mathrm{H}) .{ }^{13} \mathrm{C} \mathrm{NMR}\left(101 \mathrm{MHz}, \mathrm{CDCl}_{3}\right) \delta 143.4$, 139.3, 137.6, 137.1, 136.2, 129.8, 128.9, 128.4, 127.2, 126.3, 42.8, 36.3, 31.0, 28.0, 23.3, 21.6, 21.3, 13.9. HR-ESI-MS m/z calcd for $\mathrm{C}_{22} \mathrm{H}_{29} \mathrm{NO}_{2} \mathrm{~S}[\mathrm{M}+\mathrm{H}]^{+}$: 372.1992 , found: 372.1993 .<smiles>CCCC=C(CCCNS)c1ccc(Cl)cc1</smiles>

4-Methyl- $\boldsymbol{N}$-(4-(p-tolyl)oct-4-en-1-yl)benzenesulfonamide $\quad(\mathbf{4 c}) \quad(E / Z=2: 1)$ : Prepared according to general procedure. Step 1 and Step 2: Flash column chromatography (eluent: PE/EtOAc $=20: 1$ to $\mathrm{PE} /$ EtOAc $=6: 1, \mathrm{v} / \mathrm{v})$ to afford $\mathbf{4 c}(415$ $\mathrm{mg}, 88 \%)$ as a colorless oil. E-isomer, ${ }^{1} \mathrm{H} \mathrm{NMR}\left(400 \mathrm{MHz}, \mathrm{CDCl}_{3}\right) \delta 7.70(\mathrm{~d}, J=8.3$ $\mathrm{Hz}, 2 \mathrm{H}), 7.28(\mathrm{~d}, J=8.0 \mathrm{~Hz}, 3 \mathrm{H}), 7.25(\mathrm{~d}, J=8.6 \mathrm{~Hz}, 2 \mathrm{H}), 6.96(\mathrm{~d}, J=8.4 \mathrm{~Hz}, 2 \mathrm{H})$, $5.39(\mathrm{t}, J=7.3 \mathrm{~Hz}, 1 \mathrm{H}), 4.64(\mathrm{t}, J=6.0 \mathrm{~Hz}, 1 \mathrm{H}), 2.87(\mathrm{t}, J=6.7 \mathrm{~Hz}, 2 \mathrm{H}), 2.42(\mathrm{~s}, 3 \mathrm{H})$, $2.29(\mathrm{t}, J=7.3 \mathrm{~Hz}, 2 \mathrm{H}), 1.83(\mathrm{q}, J=7.3 \mathrm{~Hz}, 2 \mathrm{H}), 1.48-1.37(\mathrm{~m}, 2 \mathrm{H}), 1.34-1.23(\mathrm{~m}$, $2 \mathrm{H}), 0.80(\mathrm{t}, J=7.4 \mathrm{~Hz}, 3 \mathrm{H}) .{ }^{13} \mathrm{C} \mathrm{NMR}\left(101 \mathrm{MHz}, \mathrm{CDCl}_{3}\right) \delta 143.5,139.1,138.3$, 137.1, 132.4, 130.8, 129.8, 129.4, 128.4, 127.6, 127.2, 42.7, 36.1, 31.0, 27.9, 23.1, 21.6, 13.9. HR-ESI-MS $\mathrm{m} / \mathrm{z}$ calcd for $\mathrm{C}_{21} \mathrm{H}_{26} \mathrm{ClNO}_{2} \mathrm{~S}[\mathrm{M}+\mathrm{H}]^{+}: 392.1446$, found: 392.1448 .<smiles>CC(=CCCc1ccccc1)CCCN[SbH3]</smiles>

4-Methyl- $\boldsymbol{N}$-(4-methyl-7-phenylhept-4-en-1-yl)benzenesulfonamide $(4 \mathrm{~d}) \quad(E / Z=$ 2:1): Prepared according to general procedure. Step 1 and Step 2: Flash column chromatography (eluent: PE/EtOAc $=30: 1$ to PE/EtOAc $=7: 1, \mathrm{v} / \mathrm{v})$ to afford $\mathbf{4 d}(222$ $\mathrm{mg}, 31 \%)$ as a colorless oil. E-isomer, ${ }^{1} \mathrm{H} \mathrm{NMR}\left(400 \mathrm{MHz}, \mathrm{CDCl}_{3}\right) \delta 7.74(\mathrm{~d}, J=8.2$ $\mathrm{Hz}, 2 \mathrm{H}), 7.28(\mathrm{~m}, 5 \mathrm{H}), 7.15(\mathrm{~d}, J=6.9 \mathrm{~Hz}, 2 \mathrm{H}), 5.17(\mathrm{t}, J=7.1 \mathrm{~Hz}, 1 \mathrm{H}), 4.53(\mathrm{t}, J=$ $6.0 \mathrm{~Hz}, 1 \mathrm{H}), 2.85(\mathrm{dd}, J=15.3,8.5 \mathrm{~Hz}, 2 \mathrm{H}), 2.59(\mathrm{t}, J=7.7 \mathrm{~Hz}, 2 \mathrm{H}), 2.41(\mathrm{~s}, 3 \mathrm{H})$, $2.21(\mathrm{dd}, J=15.2,7.7 \mathrm{~Hz}, 2 \mathrm{H}), 1.95-1.89(\mathrm{~m}, 2 \mathrm{H}), 1.60(\mathrm{~s}, 3 \mathrm{H}), 1.44-1.38(\mathrm{~m}, 2 \mathrm{H})$. ${ }^{13} \mathrm{C}$ NMR $\left(101 \mathrm{MHz}, \mathrm{CDCl}_{3}\right) \delta 143.5,142.2,137.1,134.7,129.8,128.6,128.4,127.2$, 125.9, 125.4, 43.3, 36.3, 30.0, 28.9, 27.8, 23.3, 21.6. HR-ESI-MS m/z calcd for $\mathrm{C}_{21} \mathrm{H}_{27} \mathrm{NO}_{2} \mathrm{~S}[\mathrm{M}+\mathrm{H}]^{+}:$358.1835, found: 358.1836 .<smiles>C/C=C(\CCCN[Sn])c1ccccc1</smiles>

4-Methyl- $N$-(4-phenylhex-4-en-1-yl)benzenesulfonamide (4e) $(E / Z=5: 1)$ : Prepared according to general procedure. Step 1 and Step 2: Flash column chromatography 
(eluent: $\mathrm{PE} / \mathrm{EtOAc}=20: 1$ to $\mathrm{PE} / \mathrm{EtOAc}=6: 1, \mathrm{v} / \mathrm{v})$ to afford $4 \mathrm{e}(258 \mathrm{mg}, 65 \%)$ as a colorless oil. $E$-isomer, ${ }^{1} \mathrm{H}$ NMR $\left(400 \mathrm{MHz}, \mathrm{CDCl}_{3}\right) \delta 7.71(\mathrm{~d}, J=8.2 \mathrm{~Hz}, 2 \mathrm{H}), 7.32-$ $7.18(\mathrm{~m}, 5 \mathrm{H}), 7.04(\mathrm{~d}, J=7.0 \mathrm{~Hz}, 2 \mathrm{H}), 5.46(\mathrm{q}, J=6.8 \mathrm{~Hz}, 1 \mathrm{H}), 4.74(\mathrm{t}, J=6.1 \mathrm{~Hz}$, $1 \mathrm{H}), 2.92-2.83(\mathrm{~m}, 2 \mathrm{H}), 2.41(\mathrm{~s}, 3 \mathrm{H}), 2.31(\mathrm{t}, J=7.3 \mathrm{~Hz}, 2 \mathrm{H}), 1.50(\mathrm{~d}, J=6.8 \mathrm{~Hz}$, $3 \mathrm{H}), 1.46-1.37(\mathrm{~m}, 2 \mathrm{H}) .{ }^{13} \mathrm{C}$ NMR $\left(101 \mathrm{MHz}, \mathrm{CDCl}_{3}\right) \delta 143.3,140.3,140.1,137.0$, 129.7, 128.5, 128.2, 127.2, 126.6, 122.4, 42.7, 36.0, 27.9, 21.6, 14.7. HR-ESI-MS m/z calcd for $\mathrm{C}_{19} \mathrm{H}_{23} \mathrm{NO}_{2} \mathrm{~S}[\mathrm{M}+\mathrm{H}]^{+}: 330.1522$, found: 330.1523 .

$\mathrm{Ph}_{\mathrm{NHTS}}$

4-Methyl- $N$-(8-phenyloct-5-en-1-yl)benzenesulfonamide (4f) $(E / Z=6: 1)$ : Prepared according to general procedure. Step 1 and Step 2: Flash column chromatography (eluent: PE/EtOAc $=20: 1$ to PE/EtOAc $=6: 1, \mathrm{v} / \mathrm{v})$ to afford $\mathbf{4 f}(332 \mathrm{mg}, 78 \%)$ as a colorless oil. E-isomer, ${ }^{1} \mathrm{H} \mathrm{NMR}\left(400 \mathrm{MHz}, \mathrm{CDCl}_{3}\right) \delta 7.75(\mathrm{~d}, J=8.2 \mathrm{~Hz}, 2 \mathrm{H}), 7.32-$ $7.23(\mathrm{~m}, 4 \mathrm{H}), 7.17(\mathrm{~m}, 3 \mathrm{H}), 5.44-5.34(\mathrm{~m}, 1 \mathrm{H}), 5.32-5.23(\mathrm{~m}, 1 \mathrm{H}), 4.56(\mathrm{t}, J=5.9$ $\mathrm{Hz}, 1 \mathrm{H}), 2.93-2.83(\mathrm{~m}, 2 \mathrm{H}), 2.63(\mathrm{t}, J=7.5 \mathrm{~Hz}, 2 \mathrm{H}), 2.42(\mathrm{~s}, 3 \mathrm{H}), 2.30$ (q, $J=7.5$ $\mathrm{Hz}, 2 \mathrm{H}), 1.90(\mathrm{q}, J=7.0 \mathrm{~Hz}, 2 \mathrm{H}), 1.42-1.32(\mathrm{~m}, 2 \mathrm{H}), 1.25-1.16(\mathrm{~m}, 2 \mathrm{H}) .{ }^{13} \mathrm{C} \mathrm{NMR}$ $\left(101 \mathrm{MHz}, \mathrm{CDCl}_{3}\right) \delta 143.4,142.1,137.1,137.1,129.8,129.4,128.6,128.4,127.2$, 125.9, 43.2, 36.0, 29.3, 29.1, 26.7, 26.5, 21.6. HR-ESI-MS m/z calcd for $\mathrm{C}_{21} \mathrm{H}_{27} \mathrm{NO}_{2} \mathrm{~S}$ $[\mathrm{M}+\mathrm{H}]^{+}: 358.1835$, found: 358.1836 .

\subsection{Condition optimization for cyclization of olefinic alcohol 1a}<smiles>CCCC(=CCCc1ccccc1)CCCO</smiles>

1a

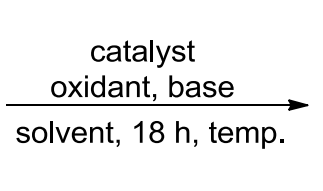
$\underset{\text { solvent, } 18 \mathrm{~h} \text {, temp. }}{\stackrel{\text { oxidant, base }}{\longrightarrow}}$<smiles>C1CCOC1</smiles>

A 4-mL vial equipped with a magnetic stir bar was charged with oxidant $(0.12 \mathrm{mmol})$, base (0.1 mmol), PhSeSePh (3.1 mg, $0.01 \mathrm{mmol}, 10 \mathrm{~mol} \%)$ and olefinic alcohol 1a (26.6 mg, $0.1 \mathrm{mmol})$. Then solvent $(2 \mathrm{~mL})$ was added, and the vial was capped. After stirred at room temperature for $18 \mathrm{~h}$, the resulting mixture was concentrated in vacuo and analyzed by ${ }^{1} \mathrm{H}$ NMR spectroscopy in $\mathrm{CDCl}_{3}$.

Table S1 Condition Optimization

\begin{tabular}{|c|c|c|c|c|c|c|c|c|c|}
\hline \multirow{2}{*}{ Entry } & \multirow{2}{*}{ Solvent } & \multirow{2}{*}{ Oxidant } & \multirow{2}{*}{ Catalyst } & \multirow{2}{*}{$\begin{array}{l}\text { Catalyst } \\
\text { Loading }\end{array}$} & \multirow{2}{*}{ Temp. } & \multirow{2}{*}{ Base } & \multirow{2}{*}{$\begin{array}{c}\text { Residual } \\
\mathbf{1 a}\end{array}$} & \multicolumn{2}{|c|}{ Yield $^{a}$} \\
\hline & & & & & & & & $2 \mathbf{a}$ & $\mathbf{3 a}$ \\
\hline 1 & $\mathrm{MeCN}$ & $\mathrm{PhI}(\mathrm{OAc})_{2}$ & $(\mathrm{PhSe})_{2}$ & $10 \%$ & $\mathrm{rt}$ & - & $73 \%$ & - & - \\
\hline 2 & $\mathrm{MeCN}$ & $\mathrm{PhI}\left(\mathrm{OCOCF}_{3}\right)_{2}$ & $(\mathrm{PhSe})_{2}$ & $10 \%$ & $\mathrm{rt}$ & - & $37 \%$ & - & - \\
\hline 3 & $\mathrm{MeCN}$ & FP-OTf & $(\mathrm{PhSe})_{2}$ & $10 \%$ & $\mathrm{rt}$ & - & trace & $64 \%$ & $15 \%$ \\
\hline 4 & $\mathrm{MeCN}$ & Selectfluor & $(\mathrm{PhSe})_{2}$ & $10 \%$ & $\mathrm{rt}$ & - & - & $16 \%$ & - \\
\hline 5 & $\mathrm{THF}$ & NFSI & $(\mathrm{PhSe})_{2}$ & $10 \%$ & $\mathrm{rt}$ & - & - & $74 \%$ & $19 \%$ \\
\hline 6 & $\mathrm{MeCN}$ & NFSI & $(\mathrm{PhSe})_{2}$ & $10 \%$ & $\mathrm{rt}$ & - & - & $59 \%$ & $19 \%$ \\
\hline 7 & $\mathrm{MeCN}$ & UHP & $(\mathrm{PhSe})_{2}$ & $10 \%$ & $\mathrm{rt}$ & - & $63 \%$ & $23 \%$ & - \\
\hline 8 & $\mathrm{MeCN}$ & $\left(\mathrm{NH}_{4}\right)_{2} \mathrm{~S}_{2} \mathrm{O}_{8}$ & $(\mathrm{PhSe})_{2}$ & $10 \%$ & $\mathrm{rt}$ & - & $92 \%$ & - & - \\
\hline 9 & $\mathrm{MeCN}$ & $\left(\mathrm{NH}_{4}\right)_{2} \mathrm{~S}_{2} \mathrm{O}_{8}$ & $(\mathrm{PhSe})_{2}$ & $10 \%$ & $60{ }^{\circ} \mathrm{C}$ & - & $47 \%$ & $4 \%$ & - \\
\hline
\end{tabular}




$\begin{array}{cccccccccc}9 & \text { THF } & \text { NFSI } & (\mathrm{PhSe})_{2} & 10 \% & \mathrm{rt} & \mathrm{NaF} & - & 78 \% & \text { trace } \\ 10 & \mathrm{MeCN} & \text { NFSI } & (\mathrm{PhSe})_{2} & 10 \% & \mathrm{rt} & \mathrm{NaF} & - & 77 \% & \text { trace } \\ 11 & \mathrm{MeCN} & \text { FP-OTf } & (\mathrm{PhSe})_{2} & 10 \% & \mathrm{rt} & \mathrm{NaF} & - & 92 \% & - \\ 12 & \mathrm{MeCN} & \text { FP-OTf } & (\mathrm{PhSe})_{2} & 10 \% & \mathrm{rt} & {\mathrm{KF} \cdot 2 \mathrm{H}_{2} 0}^{2} & 35 \% & 59 \% & - \\ 13 & \mathrm{MeCN} & \text { FP-OTf } & (\mathrm{PhSe})_{2} & 10 \% & \mathrm{rt} & \mathrm{Na}_{2} \mathrm{CO}_{3} & 29 \% & 67 \% & - \\ 14 & \mathrm{MeCN} & \text { FP-OTf } & (\mathrm{PhSe})_{2} & 10 \% & \mathrm{rt} & \mathrm{K}_{2} \mathrm{CO}_{3} & 70 \% & 29 \% & - \\ 15 & \mathrm{MeCN} & \text { FP-OTf } & (\mathrm{PhSe})_{2} & 10 \% & \mathrm{rt} & \mathrm{NaHCO}_{3} & 18 \% & 79 \% & - \\ 16 & \mathrm{MeCN} & \text { FP-OTf } & (\mathrm{PhSe})_{2} & 10 \% & \mathrm{rt} & \mathrm{pyridine} & 74 \% & 15 \% & - \\ 17 & \mathrm{MeCN} & \text { FP-OTf } & (\mathrm{PhSe})_{2} & 10 \% & \mathrm{rt} & \mathrm{Et}{ }_{3} \mathrm{~N} & 98 \% & - & - \\ 18 & \mathrm{THF} & \text { FP-OTf } & (\mathrm{PhSe})_{2} & 10 \% & \mathrm{rt} & \mathrm{NaF} & - & 91 \% & - \\ 19 & \mathrm{DCM} & \text { FP-OTf } & (\mathrm{PhSe})_{2} & 10 \% & \mathrm{rt} & \mathrm{NaF} & - & 83 \% & - \\ 20 & \text { toluene } & \text { FP-OTf } & (\mathrm{PhSe})_{2} & 10 \% & \mathrm{rt} & \mathrm{NaF} & 74 \% & 26 \% & - \\ 21 & \mathrm{CH} \mathrm{H}_{2} & \text { FP-OTf } & (\mathrm{PhSe})_{2} & 10 \% & \mathrm{rt} & \mathrm{NaF} & - & 82 \% & - \\ 22 & \mathrm{MeCN} & \text { FP-OTf } & \left(\mathrm{PhCH} \mathrm{Se}_{2}\right. & 10 \% & \mathrm{rt} & \mathrm{NaF} & 67 \% & 5 \% & - \\ 23 & \mathrm{MeCN} & \text { FP-OTf } & \mathrm{Ph} \mathrm{Se}_{2} & 10 \% & \mathrm{rt} & \mathrm{NaF} & 100 \% & - & - \\ 24 & \mathrm{MeCN} & \text { FP-OTf } & (\mathrm{PhS})_{2} & 10 \% & \mathrm{rt} & \mathrm{NaF} & 100 \% & - & - \\ 25 & \mathrm{MeCN} & \text { FP-OTf } & (\mathrm{PhSe})_{2} & 5 \% & \mathrm{rt} & \mathrm{NaF} & - & 92 \% & - \\ 26 & \mathrm{MeCN} & \text { FP-OTf } & - & - & \mathrm{rt} & \mathrm{NaF} & 72 \% & - & - \\ 29^{b} & \mathrm{MeCN} & \text { FP-OTf } & (\mathrm{PhSe})_{2} & 5 \% & \mathrm{rt} & \mathrm{NaF} & - & 98 \% & - \\ & & & & & & & (96 \%)^{c} & -\end{array}$

Conditions: 1a, $0.1 \mathrm{mmol}$; oxidant, $0.12 \mathrm{mmol}$; base, $0.1 \mathrm{mmol}$; solvent, $2.0 \mathrm{~mL} .{ }^{a}$ Determined by 400MHz NMR with 1,3,5-trimethoxybenzene as the internal standard. ${ }^{b}$ Solvent, $5.0 \mathrm{~mL} .{ }^{c}$ Isolated yield is in the parenthesis.

\subsection{Acid-facilitated rearrangement of tetrahydrofuran $2 a$}

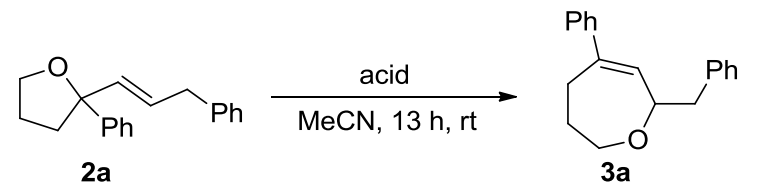

A 4-mL vial equipped with a magnetic stir bar was charged with $\mathbf{2 a}(0.05 \mathrm{mmol})$ and MeCN $(1 \mathrm{~mL})$. Then acid $(0.025 \mathrm{mmol})$ was added, and the vial was capped. After stirred at room temperature for $13 \mathrm{~h}$, the resulting mixture was concentrated in vacuo and analyzed by ${ }^{1} \mathrm{H}$ NMR spectroscopy in $\mathrm{CDCl}_{3}$.

Table S2 Rearrangement of Tetrahydrofuran 2a by Acid

\begin{tabular}{ccccc}
\hline entry & Solvent & acid & Residual 1 & Yield $^{a}$ \\
\hline 1 & MeCN & TfOH & - & - \\
2 & MeCN & $\mathrm{MsOH}$ & $65 \%$ & $16 \%$ \\
3 & MeCN & TsOH & $57 \%$ & $15 \%$ \\
4 & MeCN & TFA & $82 \%$ & $18 \%$ \\
Conditions: $2 \mathbf{2 a}, 0.05$ mmol; acid, 0.025 mmol; solvent, $1.0 \mathrm{~mL}^{a}$ Determined \\
by 400Mhz NMR with 1,3,5-trimethoxybenzene as the internal standard. \\
\hline
\end{tabular}




\subsection{Synthesis of tetrahydrofurans or-pyrans from olefinic alcohols}
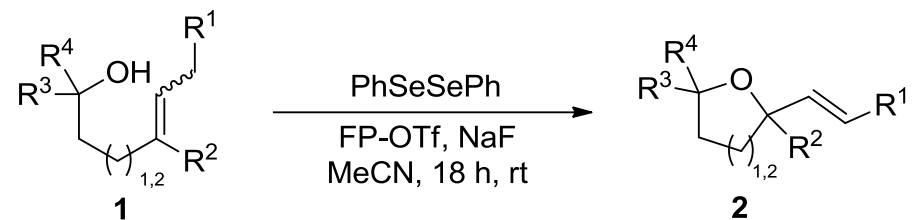

General procedure for cyclization of olefinic alcohols 1a-1r:

A 20-mL vial equipped with a magnetic stir bar was charged with FP-OTf (29.7 mg, $0.12 \mathrm{mmol}, 1.2$ euqiv), $\mathrm{NaF}$ (4.2 mg, $0.1 \mathrm{mmol}, 1$ equiv), $\mathrm{PhSeSePh}$ (1.6 mg, 0.005 mmol, $5 \mathrm{~mol} \%)$ and olefinic alcohols $1(0.1 \mathrm{mmol})$. Then dried acetonitrile $(5 \mathrm{~mL})$ was added, and the vial was capped. After stirred at room temperature for $18 \mathrm{~h}$, the resulting mixture was concentrated in vacuo. The residue was purified by silica gel column chromatography to afford the corresponding tetrahydrofurans or -pyrans 2 .<smiles>C(=CC1(c2ccccc2)CCCO1)c1ccccc1</smiles>

(E)-2-Phenyl-2-(3-phenylprop-1-en-1-yl)tetrahydrofuran (2a): Prepared according to general procedure. Flash column chromatography (eluent: PE/EtOAc $=30: 1, \mathrm{v} / \mathrm{v}$ ) to afford 2a $(25.3 \mathrm{mg}, 96 \%)$ as a colorless oil. ${ }^{1} \mathrm{H}$ NMR $\left(400 \mathrm{MHz}, \mathrm{CDCl}_{3}\right) \delta 7.45(\mathrm{~d}$, $J=7.1 \mathrm{~Hz}, 2 \mathrm{H}), 7.36(\mathrm{t}, J=7.6 \mathrm{~Hz}, 2 \mathrm{H}), 7.31(\mathrm{t}, J=7.3 \mathrm{~Hz}, 2 \mathrm{H}), 7.28(\mathrm{~d}, J=4.8 \mathrm{~Hz}$, $1 \mathrm{H}), 7.23(\mathrm{~d}, J=7.4 \mathrm{~Hz}, 1 \mathrm{H}), 7.18(\mathrm{~d}, J=7.0 \mathrm{~Hz}, 2 \mathrm{H}), 5.82(\mathrm{~d}, J=15.4 \mathrm{~Hz}, 1 \mathrm{H}), 5.74$ $(\mathrm{dt}, J=15.3,6.3 \mathrm{~Hz}, 1 \mathrm{H}), 4.06(\mathrm{td}, J=8.0,6.1 \mathrm{~Hz}, 1 \mathrm{H}), 4.00(\mathrm{td}, J=7.9,6.4 \mathrm{~Hz}, 1 \mathrm{H})$, $3.40(\mathrm{~d}, J=6.3 \mathrm{~Hz}, 2 \mathrm{H}), 2.31-2.16(\mathrm{~m}, 2 \mathrm{H}), 2.08-1.96(\mathrm{~m}, 1 \mathrm{H}), 1.94-1.82(\mathrm{~m}$, 1H). ${ }^{13} \mathrm{C}$ NMR $\left(101 \mathrm{MHz} \mathrm{CDCl}_{3}\right) \delta 145.6,140.5,136.7,128.6,128.5,128.2,128.0$, 126.7, 126.1, 125.7, 86.4, 67.7, 38.7, 38.4, 25.6. HR-ESI-MS m/z calcd for $\mathrm{C}_{19} \mathrm{H}_{20} \mathrm{O}$ $[\mathrm{M}+\mathrm{H}]^{+}: 265.1587$, found: 265.1593 .

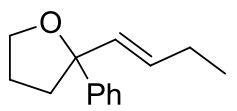

(E)-2-(But-1-en-1-yl)-2-phenyltetrahydrofuran (2b): Prepared according to general procedure. Flash column chromatography (eluent: $\mathrm{PE} / \mathrm{DCM}=6: 1, \mathrm{v} / \mathrm{v}$ ) to afford $\mathbf{2 b}$ (16.2 mg, 80\%) as a pale yellow oil. ${ }^{1} \mathrm{H}$ NMR $\left(400 \mathrm{MHz}, \mathrm{CDCl}_{3}\right) \delta 7.40(\mathrm{~d}, J=8.1$ $\mathrm{Hz}, 2 \mathrm{H}), 7.32$ (t, $J=7.6 \mathrm{~Hz}, 2 \mathrm{H}), 7.22(\mathrm{t}, J=7.2 \mathrm{~Hz}, 1 \mathrm{H}), 5.67$ (d, $J=15.5 \mathrm{~Hz}, 1 \mathrm{H})$, $5.55(\mathrm{dt}, J=15.5,6.1 \mathrm{~Hz}, 1 \mathrm{H}), 4.03(\mathrm{td}, J=8.0,6.1 \mathrm{~Hz}, 1 \mathrm{H}), 3.95(\mathrm{td}, J=8.0,6.2 \mathrm{~Hz}$, $1 \mathrm{H}), 2.25-2.13(\mathrm{~m}, 2 \mathrm{H}), 2.08-1.92(\mathrm{~m}, 3 \mathrm{H}), 1.89-1.78(\mathrm{~m}, 1 \mathrm{H}), 0.96(\mathrm{t}, J=7.4 \mathrm{~Hz}$ $3 \mathrm{H}) .{ }^{13} \mathrm{C} \mathrm{NMR}\left(101 \mathrm{MHz}, \mathrm{CDCl}_{3}\right) \delta 145.9,134.0,131.4,128.1,126.6,125.7,86.5$, 67.6, 38.4, 25.6, 25.4, 13.6. HR-ESI-MS m/z calcd for $\mathrm{C}_{14} \mathrm{H}_{18} \mathrm{O}[\mathrm{M}+\mathrm{H}]^{+}:$: 203.1430, found: 203.1429 .

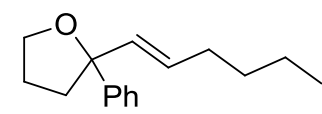

(E)-2-(Hex-1-en-1-yl)-2-phenyltetrahydrofuran (2c): Prepared according to general procedure. Flash column chromatography (eluent: $\mathrm{PE} / \mathrm{DCM}=7: 1, \mathrm{v} / \mathrm{v}$ ) to afford 2c (21.0 mg, 91\%) as a colorless oil. ${ }^{1} \mathrm{H}$ NMR $\left(400 \mathrm{MHz}, \mathrm{CDCl}_{3}\right) \delta 7.41(\mathrm{~d}, J=7.1 \mathrm{~Hz}$, 2H), $7.33(\mathrm{t}, J=7.6 \mathrm{~Hz}, 2 \mathrm{H}), 7.23(\mathrm{t}, J=7.2 \mathrm{~Hz}, 1 \mathrm{H}), 5.68(\mathrm{~d}, J=15.4 \mathrm{~Hz}, 1 \mathrm{H}), 5.53$ 
$(\mathrm{dt}, J=15.4,6.7 \mathrm{~Hz}, 1 \mathrm{H}), 4.03(\mathrm{td}, J=8.0,6.1 \mathrm{~Hz}, 1 \mathrm{H}), 3.97(\mathrm{td}, J=7.9,6.3 \mathrm{~Hz}, 1 \mathrm{H})$, $2.26-2.12(\mathrm{~m}, 2 \mathrm{H}), 2.08-1.93(\mathrm{~m}, 3 \mathrm{H}), 1.91-1.79(\mathrm{~m}, 1 \mathrm{H}), 1.40-1.23(\mathrm{~m}, 4 \mathrm{H})$, $0.88(\mathrm{t}, J=7.1 \mathrm{~Hz}, 3 \mathrm{H}) .{ }^{13} \mathrm{C} \mathrm{NMR}\left(101 \mathrm{MHz}, \mathrm{CDCl}_{3}\right) \delta 145.9,134.9,129.9,128.1$, 126.6, 125.7, 86.4, 67.6, 38.4, 32.1, 31.5, 25.5, 22.4, 14.1. HR-ESI-MS m/z calcd for $\mathrm{C}_{16} \mathrm{H}_{22} \mathrm{O}[\mathrm{M}+\mathrm{H}]^{+}: 231.1743$, found: 231.1743 .<smiles>CC1(/C=C/Cc2ccccc2)CCCO1</smiles>

(E)-2-Methyl-2-(3-phenylprop-1-en-1-yl)tetrahydrofuran (2d): Prepared according to general procedure. Flash column chromatography (eluent: PE/EtOAc $=30: 1, \mathrm{v} / \mathrm{v}$ ) to afford $2 \mathbf{d}(16.6 \mathrm{mg}, 82 \%)$ as a colorless oil. ${ }^{1} \mathrm{H} \mathrm{NMR}\left(400 \mathrm{MHz}, \mathrm{CDCl}_{3}\right) \delta 7.29(\mathrm{t}$, $J=7.3 \mathrm{~Hz}, 2 \mathrm{H}), 7.19(\mathrm{t}, J=8.1 \mathrm{~Hz}, 3 \mathrm{H}), 5.76(\mathrm{dt}, J=15.4,6.8 \mathrm{~Hz}, 1 \mathrm{H}), 5.58(\mathrm{~d}, J=$ $15.4 \mathrm{~Hz}, 1 \mathrm{H}), 3.92-3.80(\mathrm{~m}, 2 \mathrm{H}), 3.37$ (d, $J=6.7 \mathrm{~Hz}, 2 \mathrm{H}), 1.97-1.83(\mathrm{~m}, 3 \mathrm{H}), 1.75$ $-1.66(\mathrm{~m}, 1 \mathrm{H}), 1.32(\mathrm{~s}, 3 \mathrm{H}) .{ }^{13} \mathrm{C}$ NMR $\left(101 \mathrm{MHz}, \mathrm{CDCl}_{3}\right) \delta 140.7,137.2,128.6$, 128.5, 126.5, 126.1, 82.2, 67.2, 38.7, 37.7, 26.7, 25.9. HR-ESI-MS m/z calcd for $\mathrm{C}_{14} \mathrm{H}_{18} \mathrm{O}[\mathrm{M}+\mathrm{H}]^{+}:$203.1430, found: 203.1430 .

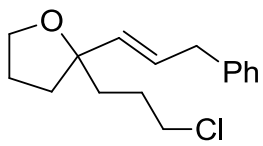

(E)-2-(3-Chloropropyl)-2-(3-phenylprop-1-en-1-yl)tetrahydrofuran (2e): Prepared according to general procedure. Flash column chromatography (eluent: PE/DCM=1:1, v/v) to afford $2 \mathrm{e}(22.9 \mathrm{mg}, 86 \%)$ as a colorless oil. ${ }^{1} \mathrm{H} \mathrm{NMR}\left(400 \mathrm{MHz}, \mathrm{CDCl}_{3}\right) \delta$ $7.30(\mathrm{t}, J=7.3 \mathrm{~Hz}, 2 \mathrm{H}), 7.23-7.15(\mathrm{~m}, 3 \mathrm{H}), 5.77(\mathrm{dt}, J=15.4,6.8 \mathrm{~Hz}, 1 \mathrm{H}), 5.46(\mathrm{~d}$, $J=15.4 \mathrm{~Hz}, 1 \mathrm{H}), 3.88-3.81(\mathrm{~m}, 2 \mathrm{H}), 3.61-3.49(\mathrm{~m}, 2 \mathrm{H}), 3.39(\mathrm{~d}, J=6.8 \mathrm{~Hz}, 2 \mathrm{H})$, $1.94-1.80(\mathrm{~m}, 5 \mathrm{H}), 1.77-1.67(\mathrm{~m}, 3 \mathrm{H}) .{ }^{13} \mathrm{C} \mathrm{NMR}\left(101 \mathrm{MHz}, \mathrm{CDCl}_{3}\right) \delta 140.7,135.6$, 128.6, 128.5, 127.7, 126.1, 84.4, 67.7, 45.8, 38.7, 37.6, 36.5, 28.1, 25.5. HR-ESI-MS $\mathrm{m} / \mathrm{z}$ calcd for $\mathrm{C}_{16} \mathrm{H}_{21} \mathrm{ClO}[\mathrm{M}+\mathrm{Na}]^{+}: 281.1173$, found: 281.1170 .<smiles>CC1(/C=C/CCCCOCc2ccccc2)CCCO1</smiles>

(E)-2-(6-(Benzyloxy)hex-1-en-1-yl)-2-methyltetrahydrofuran (2f): $\quad$ Prepared according to general procedure. Flash column chromatography (eluent: PE/EtOAc $=$ 25:1, v/v) to afford $2 \mathbf{f}(24.1 \mathrm{mg}, 88 \%)$ as a colorless oil. ${ }^{1} \mathrm{H} \mathrm{NMR}\left(400 \mathrm{MHz}, \mathrm{CDCl}_{3}\right)$ $\delta 7.37-7.27(\mathrm{~m}, 5 \mathrm{H}), 5.57(\mathrm{dt}, J=15.4,6.5 \mathrm{~Hz}, 1 \mathrm{H}), 5.47(\mathrm{~d}, J=15.5 \mathrm{~Hz}, 1 \mathrm{H}), 4.50$ (s, 2H), $3.90-3.78(\mathrm{~m}, 2 \mathrm{H}), 3.47(\mathrm{t}, J=6.6 \mathrm{~Hz}, 2 \mathrm{H}), 2.03(\mathrm{dd}, J=14.0,6.7 \mathrm{~Hz}, 2 \mathrm{H})$, $1.95-1.79(\mathrm{~m}, 3 \mathrm{H}), 1.70-1.57(\mathrm{~m}, 3 \mathrm{H}), 1.50-1.41(\mathrm{~m}, 2 \mathrm{H}), 1.29(\mathrm{~s}, 3 \mathrm{H}) .{ }^{13} \mathrm{C}$ NMR $\left(101 \mathrm{MHz}, \mathrm{CDCl}_{3}\right) \delta 138.8,135.8,128.5,127.7,127.6,127.6,82.2,73.0,70.4,67.4$, 37.7, 32.1, 29.4, 26.7, 26.1, 25.9. HR-ESI-MS m/z calcd for $\mathrm{C}_{18} \mathrm{H}_{26} \mathrm{O}_{2}[\mathrm{M}+\mathrm{H}]^{+}$: 275.2006, found: 275.2013 .

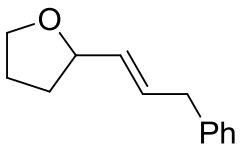

(E)-2-(3-Phenylprop-1-en-1-yl)tetrahydrofuran (2g): Prepared according to general procedure. Flash column chromatography (eluent: $\mathrm{PE} / \mathrm{EtOAc}=30: 1, \mathrm{v} / \mathrm{v}$ ) to afford $\mathbf{2 g}$ $(13.3 \mathrm{mg}, 71 \%, \mathrm{Z} / E=1: 5)$ as a colorless oil. ${ }^{1} \mathrm{H} \mathrm{NMR}\left(400 \mathrm{MHz}, \mathrm{CDCl}_{3}\right) \delta 7.29(\mathrm{t}, J$ $=7.4 \mathrm{~Hz}, 2 \mathrm{H}), 7.20(\mathrm{t}, J=6.8 \mathrm{~Hz}, 3 \mathrm{H}), 5.84(\mathrm{dt}, J=15.2,6.8 \mathrm{~Hz}, 1 \mathrm{H}), 5.54(\mathrm{dd}, J=$ 
15.3, 7.0 Hz, 1H), $4.28(\mathrm{q}, J=7.0 \mathrm{~Hz}, 1 \mathrm{H}), 3.90(\mathrm{dd}, J=14.3,7.6 \mathrm{~Hz}, 1 \mathrm{H}), 3.77$ (dd, $J=14.1,7.9 \mathrm{~Hz}, 1 \mathrm{H}), 3.38(\mathrm{~d}, J=6.7 \mathrm{~Hz}, 2 \mathrm{H}), 2.11-1.98(\mathrm{~m}, 1 \mathrm{H}), 1.99-1.83(\mathrm{~m}$, 2H), $1.67-1.55(\mathrm{~m}, 1 \mathrm{H}) .{ }^{13} \mathrm{C}$ NMR $\left(101 \mathrm{MHz}, \mathrm{CDCl}_{3}\right) \delta 140.1,132.3,130.9,128.6$, $128.4,126.1,79.7,68.0,38.6,32.2,25.9$. HR-ESI-MS m/z calcd for $\mathrm{C}_{13} \mathrm{H}_{16} \mathrm{O}[\mathrm{M}+\mathrm{H}]^{+}$: 189.1274, found: 189.1272 .<smiles>CC/C=C/C1(c2ccc(C)cc2)CCCO1</smiles>

(E)-2-(But-1-en-1-yl)-2-(p-tolyl)tetrahydrofuran (2h): Prepared according to general procedure. Flash column chromatography (eluent: $\mathrm{PE} / \mathrm{DCM}=6: 1, \mathrm{v} / \mathrm{v}$ ) to afford $2 \mathrm{~h}(16.0 \mathrm{mg}, 74 \%)$ as a colorless oil. ${ }^{1} \mathrm{H}$ NMR $\left(400 \mathrm{MHz}, \mathrm{CDCl}_{3}\right) \delta 7.28(\mathrm{~d}, J$ $=8.2 \mathrm{~Hz}, 2 \mathrm{H}), 7.14(\mathrm{~d}, J=7.9 \mathrm{~Hz}, 2 \mathrm{H}), 5.66(\mathrm{~d}, J=15.5 \mathrm{~Hz}, 1 \mathrm{H}), 5.54(\mathrm{dt}, J=15.5$, $6.1 \mathrm{~Hz}, 1 \mathrm{H}), 4.01(\mathrm{td}, J=8.0,6.2 \mathrm{~Hz}, 1 \mathrm{H}), 3.94(\mathrm{td}, J=8.0,6.1 \mathrm{~Hz}, 1 \mathrm{H}), 2.34(\mathrm{~s}, 3 \mathrm{H})$, $2.21-2.13(\mathrm{~m}, 2 \mathrm{H}), 2.08-1.93(\mathrm{~m}, 3 \mathrm{H}), 1.90-1.77(\mathrm{~m}, 1 \mathrm{H}), 0.96(\mathrm{t}, J=7.4 \mathrm{~Hz}, 3 \mathrm{H})$. ${ }^{13} \mathrm{C}$ NMR $\left(101 \mathrm{MHz}, \mathrm{CDCl}_{3}\right) \delta 142.9,136.1,134.2,131.2,128.8,125.7,86.4,67.5$, 38.4, 25.6, 25.4, 21.2, 13.6. HR-ESI-MS m/z calcd for $\mathrm{C}_{15} \mathrm{H}_{20} \mathrm{O}[\mathrm{M}+\mathrm{H}]^{+}:$217.1587, found: 217.1584 .

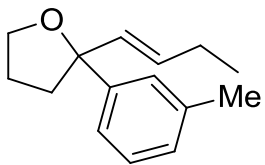

(E)-2-(But-1-en-1-yl)-2-(m-tolyl)tetrahydrofuran (2i): Prepared according to general procedure. Flash column chromatography (eluent: $\mathrm{PE} / \mathrm{DCM}=6: 1, \mathrm{v} / \mathrm{v}$ ) to afford $2 \mathbf{i}(17.7 \mathrm{mg}, 82 \%)$ as a colorless oil. ${ }^{1} \mathrm{H} \mathrm{NMR}\left(400 \mathrm{MHz}, \mathrm{CDCl}_{3}\right) \delta 7.25-7.16$ $(\mathrm{m}, 3 \mathrm{H}), 7.05(\mathrm{~d}, J=7.0 \mathrm{~Hz}, 1 \mathrm{H}), 5.67(\mathrm{~d}, J=15.5 \mathrm{~Hz}, 1 \mathrm{H}), 5.58(\mathrm{dt}, J=15.4,5.9 \mathrm{~Hz}$, $1 \mathrm{H}), 4.03(\mathrm{td}, J=8.0,6.0 \mathrm{~Hz}, 1 \mathrm{H}), 3.96(\mathrm{td}, J=7.9,6.3 \mathrm{~Hz}, 1 \mathrm{H}), 2.37$ (s, 3H), $2.26-$ $2.12(\mathrm{~m}, 2 \mathrm{H}), 2.10-1.92(\mathrm{~m}, 3 \mathrm{H}), 1.91-1.79(\mathrm{~m}, 1 \mathrm{H}), 0.98(\mathrm{t}, J=7.4 \mathrm{~Hz}, 3 \mathrm{H}) .{ }^{13} \mathrm{C}$ NMR $\left(101 \mathrm{MHz}, \mathrm{CDCl}_{3}\right) \delta 145.9,137.7,134.1,131.1,128.0,127.3,126.3,122.8$, 86.4, 67.6, 38.3, 25.5, 25.4, 21.7, 13.6. HR-ESI-MS m/z calcd for $\mathrm{C}_{15} \mathrm{H}_{20} \mathrm{O}[\mathrm{M}+\mathrm{H}]^{+}$: 217.1587, found: 217.1582 .<smiles>CCC=CC1(c2ccc(C(C)C)cc2)CCCO1</smiles>

(E)-2-(But-1-en-1-yl)-2-(4-isopropylphenyl)tetrahydrofuran $\quad(\mathbf{2 j}): \quad$ Prepared according to general procedure. Flash column chromatography (eluent: $\mathrm{PE} / \mathrm{DCM}=$ 6:1, v/v) to afford $\mathbf{2 j}(20.4 \mathrm{mg}, 83 \%)$ as a colorless oil. ${ }^{1} \mathrm{H}$ NMR $\left(400 \mathrm{MHz}, \mathrm{CDCl}_{3}\right) \delta$ $7.31(\mathrm{~d}, J=8.3 \mathrm{~Hz}, 2 \mathrm{H}), 7.18(\mathrm{~d}, J=8.1 \mathrm{~Hz}, 2 \mathrm{H}), 5.66(\mathrm{~d}, J=15.5 \mathrm{~Hz}, 1 \mathrm{H}), 5.58(\mathrm{dt}$, $J=15.4,5.9 \mathrm{~Hz}, 1 \mathrm{H}), 4.02(\mathrm{td}, J=8.0,6.0 \mathrm{~Hz}, 1 \mathrm{H}), 3.95(\mathrm{td}, J=7.9,6.3 \mathrm{~Hz}, 1 \mathrm{H})$, 2.90 (hept, $J=6.9 \mathrm{~Hz}, 1 \mathrm{H}), 2.24-2.12(\mathrm{~m}, 2 \mathrm{H}), 2.09-1.92(\mathrm{~m}, 3 \mathrm{H}), 1.91-1.79(\mathrm{~m}$, $1 \mathrm{H}), 1.25(\mathrm{~d}, J=6.9 \mathrm{~Hz}, 6 \mathrm{H}), 0.97(\mathrm{t}, J=7.4 \mathrm{~Hz}, 3 \mathrm{H}) .{ }^{13} \mathrm{C} \mathrm{NMR}\left(101 \mathrm{MHz}, \mathrm{CDCl}_{3}\right) \delta$ 147.0, 143.2, 134.1, 130.9, 126.1, 125.6, 86.4, 67.6, 38.3, 33.8, 25.6, 25.4, 24.1, 13.6. HR-ESI-MS m/z calcd for $\mathrm{C}_{17} \mathrm{H}_{24} \mathrm{O}[\mathrm{M}+\mathrm{H}]^{+}: 245.1900$, found: 245.1892 . 
<smiles>FC(F)(F)c1ccc(C2(c3ccccc3)CCCO2)cc1</smiles>

(E)-2-(3-Phenylprop-1-en-1-yl)-2-(4-(trifluoromethyl)phenyl)tetrahydrofuran

(2k): Prepared according to general procedure. Flash column chromatography (eluent: $\mathrm{PE} / \mathrm{EtOAc}=30: 1, \mathrm{v} / \mathrm{v})$ to afford $\mathbf{2 k}(27.6 \mathrm{mg}, 83 \%)$ as a pale yellow oil. ${ }^{1} \mathrm{H}$ NMR $\left(400 \mathrm{MHz}, \mathrm{CDCl}_{3}\right) \delta 7.60(\mathrm{~d}, J=8.4 \mathrm{~Hz}, 2 \mathrm{H}), 7.54(\mathrm{~d}, J=8.4 \mathrm{~Hz}, 2 \mathrm{H}), 7.30(\mathrm{t}, J=7.3$ $\mathrm{Hz}, 2 \mathrm{H}), 7.22(\mathrm{t}, J=7.3 \mathrm{~Hz}, 1 \mathrm{H}), 7.16(\mathrm{~d}, J=7.1 \mathrm{~Hz}, 2 \mathrm{H}), 5.82-5.70(\mathrm{~m}, 2 \mathrm{H}), 4.07$ $(\mathrm{td}, J=8.0,6.1 \mathrm{~Hz}, 1 \mathrm{H}), 3.99(\mathrm{td}, J=7.9,6.4 \mathrm{~Hz}, 1 \mathrm{H}), 3.39(\mathrm{~d}, J=5.1 \mathrm{~Hz}, 2 \mathrm{H}), 2.34$ $-2.24(\mathrm{~m}, 1 \mathrm{H}), 2.22-2.11(\mathrm{~m}, 1 \mathrm{H}), 2.09-1.96(\mathrm{~m}, 1 \mathrm{H}), 1.92-1.80(\mathrm{~m}, 1 \mathrm{H}) .{ }^{13} \mathrm{C}$ NMR $\left(101 \mathrm{MHz}, \mathrm{CDCl}_{3}\right) \delta 149.87,140.17,135.87,129.28$ (q, $\left.J=32.2 \mathrm{~Hz}\right), 128.83$, 128.63, 128.56, 126.23, 126.01, 125.19 (q, $J=3.7 \mathrm{~Hz}), 124.41(\mathrm{q}, J=272.0 \mathrm{~Hz})$, 86.15, 67.90, 38.67, 38.53, 25.54. HR-ESI-MS m/z calcd for $\mathrm{C}_{20} \mathrm{H}_{19} \mathrm{~F}_{3} \mathrm{O}[\mathrm{M}+\mathrm{H}]^{+}$: 333.1461, found: 333.1460 .<smiles>CCC=CC1(c2ccc(Cl)cc2)CCCO1</smiles>

(E)-2-(But-1-en-1-yl)-2-(4-chlorophenyl)tetrahydrofuran (2l): Prepared according to general procedure. Flash column chromatography (eluent: $\mathrm{PE} / \mathrm{DCM}=4: 1, \mathrm{v} / \mathrm{v}$ ) to afford $2 \mathrm{l}(21.5 \mathrm{mg}, 91 \%)$ as a colorless oil. ${ }^{1} \mathrm{H} \mathrm{NMR}\left(400 \mathrm{MHz}, \mathrm{CDCl}_{3}\right) \delta 7.32(\mathrm{~d}, J=$ $8.8 \mathrm{~Hz}, 2 \mathrm{H}), 7.28(\mathrm{~d}, J=8.8 \mathrm{~Hz}, 2 \mathrm{H}), 5.63(\mathrm{~d}, J=15.5 \mathrm{~Hz}, 1 \mathrm{H}), 5.53(\mathrm{dt}, J=15.5,6.0$ $\mathrm{Hz}, 1 \mathrm{H}), 4.01(\mathrm{td}, J=8.0,6.2 \mathrm{~Hz}, 1 \mathrm{H}), 3.93(\mathrm{td}, J=8.0,6.1 \mathrm{~Hz}, 1 \mathrm{H}), 2.24-2.08(\mathrm{~m}$, 2H), $2.07-1.92(\mathrm{~m}, 3 \mathrm{H}), 1.89-1.76(\mathrm{~m}, 1 \mathrm{H}), 0.96(\mathrm{t}, J=7.4 \mathrm{~Hz}, 3 \mathrm{H}) .{ }^{13} \mathrm{C} \mathrm{NMR}$ $\left(101 \mathrm{MHz}, \mathrm{CDCl}_{3}\right) \delta 144.5,133.6,132.4,131.9,128.2,127.2,86.1,77.5,77.2,76.8$, 67.7, 38.5, 25.5, 25.3, 13.6. HR-ESI-MS m/z calcd for $\mathrm{C}_{14} \mathrm{H}_{17} \mathrm{ClO}[\mathrm{M}+\mathrm{H}]^{+}:$237.1041, found: 237.1036 .<smiles>CC/C=C/C1(c2ccc(Br)cc2)CCCO1</smiles>

(E)-2-(4-Bromophenyl)-2-(but-1-en-1-yl)tetrahydrofuran $\quad(2 \mathrm{~m})$ : $\quad$ Prepared according to general procedure. Flash column chromatography (eluent: PE/DCM = $5: 1, \mathrm{v} / \mathrm{v})$ to afford $\mathbf{2 m}(22.8 \mathrm{mg}, 81 \%)$ as a colorless oil. ${ }^{1} \mathrm{H} \mathrm{NMR}\left(400 \mathrm{MHz}, \mathrm{CDCl}_{3}\right)$ $\delta 7.43(\mathrm{~d}, J=8.5 \mathrm{~Hz}, 2 \mathrm{H}), 7.27(\mathrm{~d}, J=8.5 \mathrm{~Hz}, 2 \mathrm{H}), 5.62(\mathrm{~d}, J=15.5 \mathrm{~Hz}, 1 \mathrm{H}), 5.53(\mathrm{dt}$, $J=15.5,6.0 \mathrm{~Hz}, 1 \mathrm{H}), 4.01(\mathrm{td}, J=7.9,6.3 \mathrm{~Hz}, 1 \mathrm{H}), 3.93(\mathrm{td}, J=8.0,6.1 \mathrm{~Hz}, 1 \mathrm{H})$, $2.23-2.07(\mathrm{~m}, 2 \mathrm{H}), 2.07-1.92(\mathrm{~m}, 3 \mathrm{H}), 1.88-1.76(\mathrm{~m}, 1 \mathrm{H}), 0.96(\mathrm{t}, J=7.4 \mathrm{~Hz}, 3 \mathrm{H})$. ${ }^{13} \mathrm{C} \mathrm{NMR}\left(101 \mathrm{MHz}, \mathrm{CDCl}_{3}\right) \delta 145.0,133.5,131.9,131.2,127.6,120.5,86.1,67.7$, 38.4, 25.5, 25.3, 13.5. HR-ESI-MS $\mathrm{m} / \mathrm{z}$ calcd for $\mathrm{C}_{14} \mathrm{H}_{17} \mathrm{BrO}[\mathrm{M}+\mathrm{H}]^{+}$: 281.0536, found: 281.0537. 


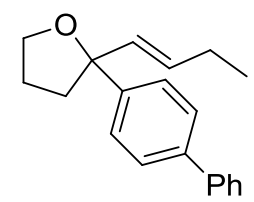

(E)-2-([1,1'-Biphenyl]-4-yl)-2-(but-1-en-1-yl)tetrahydrofuran (2n): Prepared according to general procedure. Flash column chromatography (eluent: $\mathrm{PE} / \mathrm{DCM}=$ $3: 1, \mathrm{v} / \mathrm{v})$ to afford $\mathbf{2 n}(24.4 \mathrm{mg}, 88 \%)$ as a white solid. ${ }^{1} \mathrm{H} \mathrm{NMR}\left(400 \mathrm{MHz}, \mathrm{CDCl}_{3}\right) \delta$ $7.62(\mathrm{~d}, J=7.1 \mathrm{~Hz}, 2 \mathrm{H}), 7.58(\mathrm{~d}, J=8.4 \mathrm{~Hz}, 2 \mathrm{H}), 7.49(\mathrm{~d}, J=8.5 \mathrm{~Hz}, 2 \mathrm{H}), 7.45(\mathrm{t}, J=$ $7.6 \mathrm{~Hz}, 2 \mathrm{H}), 7.35(\mathrm{t}, J=7.4 \mathrm{~Hz}, 1 \mathrm{H}), 5.72(\mathrm{~d}, J=15.5 \mathrm{~Hz}, 1 \mathrm{H}), 5.63(\mathrm{dt}, J=15.5,6.0$ $\mathrm{Hz}, 1 \mathrm{H}), 4.07(\mathrm{td}, J=8.0,6.2 \mathrm{~Hz}, 1 \mathrm{H}), 4.00(\mathrm{td}, J=8.0,6.2 \mathrm{~Hz}, 1 \mathrm{H}), 2.30-2.18(\mathrm{~m}$, $2 \mathrm{H}), 2.12-1.97(\mathrm{~m}, 3 \mathrm{H}), 1.95-1.83(\mathrm{~m}, 1 \mathrm{H}), 1.00(\mathrm{t}, J=7.4 \mathrm{~Hz}, 3 \mathrm{H}) .{ }^{13} \mathrm{C}$ NMR $\left(101 \mathrm{MHz}, \mathrm{CDCl}_{3}\right) \delta 145.0,141.1,139.5,133.9,131.5,128.8,127.2,127.2,126.9$, 126.2, 86.4, 67.7, 38.4, 25.6, 25.4, 13.6. HR-ESI-MS m/z calcd for $\mathrm{C}_{20} \mathrm{H}_{22} \mathrm{O}[\mathrm{M}+\mathrm{H}]^{+}$: 279.1743 , found: 279.1735 .

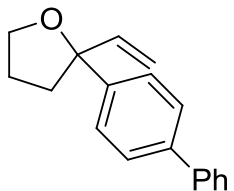

2-([1,1'-Biphenyl]-4-yl)-2-vinyltetrahydrofuran (2o): Prepared according to general procedure. Flash column chromatography (eluent: $\mathrm{PE} / \mathrm{DCM}=4: 1, \mathrm{v} / \mathrm{v}$ ) to afford 20 (18.4 mg, 74\%) as a colorless oil. ${ }^{1} \mathrm{H}$ NMR $\left(400 \mathrm{MHz}, \mathrm{CDCl}_{3}\right) \delta 7.60(\mathrm{~d}, J=7.1 \mathrm{~Hz}$, 2H), $7.57(\mathrm{~d}, J=8.5 \mathrm{~Hz}, 2 \mathrm{H}), 7.49(\mathrm{~d}, J=8.5 \mathrm{~Hz}, 2 \mathrm{H}), 7.44(\mathrm{t}, J=7.6 \mathrm{~Hz}, 2 \mathrm{H}), 7.34$ $(\mathrm{t}, J=7.4 \mathrm{~Hz}, 1 \mathrm{H}), 6.10(\mathrm{dd}, J=17.2,10.5 \mathrm{~Hz}, 1 \mathrm{H}), 5.23(\mathrm{dd}, J=17.2,1.4 \mathrm{~Hz}, 1 \mathrm{H})$, $5.12(\mathrm{dd}, J=10.5,1.4 \mathrm{~Hz}, 1 \mathrm{H}), 4.12-3.98(\mathrm{~m}, 2 \mathrm{H}), 2.33-2.17(\mathrm{~m}, 2 \mathrm{H}), 2.10-1.98$ $(\mathrm{m}, 1 \mathrm{H}), 1.98-1.86(\mathrm{~m}, 1 \mathrm{H}) .{ }^{13} \mathrm{C}$ NMR $\left(101 \mathrm{MHz}, \mathrm{CDCl}_{3}\right) \delta 144.4,143.0,141.1$, $139.8,128.9,127.3,127.2,127.0,126.1,113.0,86.6,68.0,38.0,25.5$. HR-ESI-MS $\mathrm{m} / \mathrm{z}$ calcd for $\mathrm{C}_{18} \mathrm{H}_{18} \mathrm{O}[\mathrm{M}+\mathrm{H}]^{+}: 251.1430$, found: 251.1424 .<smiles>CCC=CC1([PH2+]CCC)CCC(C)O1</smiles>

(E)-2-(But-1-en-1-yl)-5-methyl-2-phenyltetrahydrofuran (2p): Prepared according to general procedure. Flash column chromatography (eluent: $\mathrm{PE} / \mathrm{DCM}=6: 1, \mathrm{v} / \mathrm{v}$ ) to afford $\mathbf{2 p}(17.0 \mathrm{mg}, 79 \%$, d.r. $=1: 1)$ as a colorless oil. ${ }^{1} \mathrm{H}$ NMR $\left(400 \mathrm{MHz}, \mathrm{CDCl}_{3}\right) \delta$ $7.47-7.38(\mathrm{~m}, 2 \mathrm{H}), 7.35-7.29(\mathrm{~m}, 2 \mathrm{H}), 7.25-7.19(\mathrm{~m}, 1 \mathrm{H}), 5.75-5.64(\mathrm{~m}, 1 \mathrm{H})$, $5.62-5.46(\mathrm{~m}, 1 \mathrm{H}), 4.36-4.12(\mathrm{~m}, 1 \mathrm{H}), 2.31-2.15(\mathrm{~m}, 2 \mathrm{H}), 2.11-1.98(\mathrm{~m}, 2.5 \mathrm{H})$, $1.92(\mathrm{dq}, J=13.1,6.5 \mathrm{~Hz}, 0.5 \mathrm{H}), 1.61(\mathrm{dq}, J=12.0,7.6 \mathrm{~Hz}, 0.5 \mathrm{H}), 1.49(\mathrm{dq}, J=12.0$, $7.7 \mathrm{~Hz}, 0.5 \mathrm{H}), 1.34(\mathrm{dd}, J=9.0,6.1 \mathrm{~Hz}, 3 \mathrm{H}), 0.97(\mathrm{dd}, J=15.5,7.5 \mathrm{~Hz}, 3 \mathrm{H}) .{ }^{13} \mathrm{C}$ NMR $\left(101 \mathrm{MHz}, \mathrm{CDCl}_{3}\right) \delta 146.9,146.4,135.4,134.4,131.3,130.9,128.1,127.9$, $126.5,126.5,125.8,125.7,86.7,86.5,75.4,75.2,39.2,39.0,33.2,33.1,25.4,21.8$, 21.8, 13.7, 13.6. HR-ESI-MS $\mathrm{m} / \mathrm{z}$ calcd for $\mathrm{C}_{15} \mathrm{H}_{20} \mathrm{O}[\mathrm{M}+\mathrm{H}]^{+}:$217.1587, found: 217.1587.

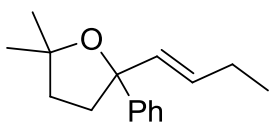


(E)-2-(But-1-en-1-yl)-5,5-dimethyl-2-phenyltetrahydrofuran (2q): $\quad$ Prepared according to general procedure. Flash column chromatography (eluent: PE/DCM=6:1, v/v) to afford $2 q(15.5 \mathrm{mg}, 67 \%)$ as a colorless oil. ${ }^{1} \mathrm{H}$ NMR $\left(400 \mathrm{MHz}, \mathrm{CDCl}_{3}\right) \delta$ $7.44(\mathrm{~d}, J=7.1 \mathrm{~Hz}, 2 \mathrm{H}), 7.31(\mathrm{t}, J=7.6 \mathrm{~Hz}, 2 \mathrm{H}), 7.21(\mathrm{t}, J=7.3 \mathrm{~Hz}, 1 \mathrm{H}), 5.68(\mathrm{~d}, J=$ $15.4 \mathrm{~Hz}, 1 \mathrm{H}), 5.51(\mathrm{dt}, J=15.4,6.3 \mathrm{~Hz}, 1 \mathrm{H}), 2.40-2.25(\mathrm{~m}, 2 \mathrm{H}), 2.08-1.98(\mathrm{~m}$, 2H), $1.85(\mathrm{dt}, J=12.1,7.0 \mathrm{~Hz}, 1 \mathrm{H}), 1.71(\mathrm{dt}, J=12.1,7.0 \mathrm{~Hz}, 1 \mathrm{H}), 1.39$ (s, 3H), 1.30 $(\mathrm{s}, 3 \mathrm{H}), 0.96(\mathrm{t}, J=7.4 \mathrm{~Hz}, 3 \mathrm{H}) .{ }^{13} \mathrm{C} \mathrm{NMR}\left(101 \mathrm{MHz}, \mathrm{CDCl}_{3}\right) \delta 147.2,135.9,130.6$, 127.9, 126.4, 125.8, 86.9, 82.0, 39.3, 38.3, 29.5, 29.1, 25.3, 13.6. HR-ESI-MS m/z calcd for $\mathrm{C}_{16} \mathrm{H}_{22} \mathrm{O}[\mathrm{M}+\mathrm{H}]^{+}$: 231.1743, found: 231.1737 .<smiles>C(=C/C1(c2ccccc2)CCCCO1)\c1ccccc1</smiles>

(E)-2-Phenyl-2-(3-Phenylprop-1-en-1-yl)tetrahydro-2H-pyran (2r): Prepared according to general procedure. Flash column chromatography (eluent: PE/EtOAc = $30: 1, \mathrm{v} / \mathrm{v})$ to afford $\mathbf{2 q}(22.0 \mathrm{mg}, 79 \%)$ as a colorless oil. ${ }^{1} \mathrm{H}$ NMR $\left(400 \mathrm{MHz}, \mathrm{CDCl}_{3}\right)$ $\delta 7.45(\mathrm{~d}, J=7.2 \mathrm{~Hz}, 2 \mathrm{H}), 7.37(\mathrm{t}, J=7.7 \mathrm{~Hz}, 2 \mathrm{H}), 7.34-7.27(\mathrm{~m}, 3 \mathrm{H}), 7.25-7.20$ $(\mathrm{m}, 1 \mathrm{H}), 7.17(\mathrm{~d}, J=6.9 \mathrm{~Hz}, 2 \mathrm{H}), 5.71-5.60(\mathrm{~m}, 2 \mathrm{H}), 3.89-3.79(\mathrm{~m}, 1 \mathrm{H}), 3.75-$ $3.66(\mathrm{~m}, 1 \mathrm{H}), 3.40(\mathrm{~d}, J=3.6 \mathrm{~Hz}, 2 \mathrm{H}), 2.20-2.08(\mathrm{~m}, 1 \mathrm{H}), 2.07-1.98(\mathrm{~m}, 1 \mathrm{H}), 1.81$ $-1.70(\mathrm{~m}, 1 \mathrm{H}), 1.70-1.48(\mathrm{~m}, 3 \mathrm{H}) .{ }^{13} \mathrm{C} \mathrm{NMR}\left(101 \mathrm{MHz}, \mathrm{CDCl}_{3}\right) \delta 144.8,140.5$, 137.9, 129.0, 128.7, 128.5, 128.4, 126.7, 126.4, 126.1, 78.2, 63.1, 39.0, 34.2, 26.1, 20.3. HR-ESI-MS m/z calcd for $\mathrm{C}_{20} \mathrm{H}_{22} \mathrm{O}[\mathrm{M}+\mathrm{H}]^{+}:$279.1743, found: 279.1734 .<smiles>C(=C/C1CCCCO1)\Cc1ccccc1</smiles>

(E)-2-(3-Phenylprop-1-en-1-yl)tetrahydro-2H-pyran (2s): Prepared according to general procedure. Flash column chromatography (eluent: $\mathrm{PE} / \mathrm{EtOAc}=30: 1, \mathrm{v} / \mathrm{v}$ ) to afford $2 \mathbf{r}(15.0 \mathrm{mg}, 74 \%, Z / E=1: 8)$ as a colorless oil. ${ }^{1} \mathrm{H} \mathrm{NMR}\left(400 \mathrm{MHz}, \mathrm{CDCl}_{3}\right) \delta$ $7.31-7.26(\mathrm{~m}, 2 \mathrm{H}), 7.19(\mathrm{t}, J=6.9 \mathrm{~Hz}, 3 \mathrm{H}), 5.88-5.78(\mathrm{~m}, 1 \mathrm{H}), 5.54(\mathrm{dd}, J=15.4$, $6.1 \mathrm{~Hz}, 1 \mathrm{H}), 4.06-3.96(\mathrm{~m}, 1 \mathrm{H}), 3.84-3.74(\mathrm{~m}, 1 \mathrm{H}), 3.53-3.42(\mathrm{~m}, 1 \mathrm{H}), 3.37(\mathrm{~d}, J$ $=6.7 \mathrm{~Hz}, 2 \mathrm{H}), 1.92-1.78(\mathrm{~m}, 1 \mathrm{H}), 1.71-1.33(\mathrm{~m}, 5 \mathrm{H}) .{ }^{13} \mathrm{C} \mathrm{NMR}\left(101 \mathrm{MHz}, \mathrm{CDCl}_{3}\right)$ $\delta 140.3,132.8,130.3,128.7,128.5,126.1,78.1,68.5,38.9,32.3,26.0,23.5$. HR-ESI-MS m/z calcd for $\mathrm{C}_{14} \mathrm{H}_{18} \mathrm{O}[\mathrm{M}+\mathrm{H}]^{+}:$203.1430, found: 203.1425 .

\subsection{Condition optimization for cyclization of olefinic sulfonamide 4 a}

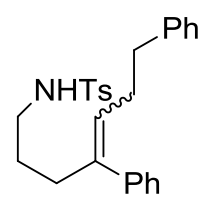

4a

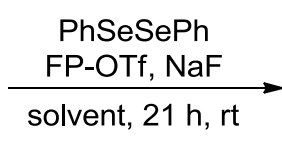

solvent, $21 \mathrm{~h}, \mathrm{rt}$

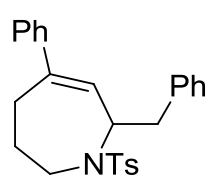

$6 a$

A 4-mL vial equipped with a magnetic stir bar was charged with FP-OTf (14.8 $\mathrm{mg}$, $0.06 \mathrm{mmol}), \mathrm{NaF}(0.025 \mathrm{mmol}$ or $0.05 \mathrm{mmol}), \mathrm{PhSeSePh}$ (1.6 mg, $0.005 \mathrm{mmol}, 10$ mol \%) and olefinic sulfonamide $4 \mathbf{a}(21.0 \mathrm{mg}, 0.05 \mathrm{mmol})$. Then solvent $(2 \mathrm{~mL})$ was added, and the vial was capped. After stirred at room temperature for $21 \mathrm{~h}$, the resulting mixture was concentrated in vacuo and analyzed by ${ }^{1} \mathrm{H}$ NMR spectroscopy 
in $\mathrm{CDCl}_{3}$.

Table S3 Condition Optimization

\begin{tabular}{|c|c|c|c|c|c|c|}
\hline \multirow{2}{*}{ Entry } & \multirow{2}{*}{ Solvent } & \multirow{2}{*}{$\mathrm{PhSeSePh}$} & \multirow{2}{*}{$\mathrm{NaF}$} & \multirow{2}{*}{$\begin{array}{c}\text { Residual } \\
\mathbf{4 a}\end{array}$} & \multicolumn{2}{|c|}{ Yield $^{a}$} \\
\hline & & & & & $5 a$ & $6 a$ \\
\hline 1 & $\mathrm{MeCN}$ & $10 \mathrm{~mol} \%$ & - & $16 \%$ & 0 & $53 \%$ \\
\hline 2 & THF & $10 \mathrm{~mol} \%$ & - & $18 \%$ & $16 \%$ & $59 \%$ \\
\hline 3 & DCM & $10 \mathrm{~mol} \%$ & - & $22 \%$ & $25 \%$ & $32 \%$ \\
\hline 4 & DCE & $10 \mathrm{mo} 1 \%$ & - & $9 \%$ & $9 \%$ & $59 \%$ \\
\hline 5 & $\mathrm{CH}_{3} \mathrm{NO}_{2}$ & $10 \mathrm{~mol} \%$ & - & $8 \%$ & $8 \%$ & $51 \%$ \\
\hline 6 & $\mathrm{DCM}$ & $10 \mathrm{~mol} \mathrm{\%}$ & 1 equiv. & $7 \%$ & $21 \%$ & $69 \%$ \\
\hline 7 & DCE & $10 \mathrm{~mol} \%$ & 1 equiv. & $4 \%$ & $95 \%$ & 0 \\
\hline 8 & DCE & $5 \mathrm{~mol} \%$ & 1 equiv. & $4 \%$ & $85 \%$ & 0 \\
\hline 9 & $\mathrm{CH}_{3} \mathrm{NO}_{2}$ & $10 \mathrm{~mol} \mathrm{\%}$ & 0.5 equiv. & $5 \%$ & $67 \%$ & $4 \%$ \\
\hline 10 & $\mathrm{MeCN}$ & $10 \mathrm{~mol} \mathrm{\%}$ & 0.5 equiv. & $37 \%$ & $51 \%$ & 0 \\
\hline 11 & THF & $10 \mathrm{~mol} \mathrm{\%}$ & 0.5 equiv. & $65 \%$ & $44 \%$ & 0 \\
\hline 12 & DCM & $10 \mathrm{~mol} \mathrm{\%}$ & 0.5 equiv. & $6 \%$ & $14 \%$ & $65 \%$ \\
\hline 13 & DCE & $10 \mathrm{~mol} \%$ & 0.5 equiv. & $3 \%$ & $5 \%$ & $77 \%$ \\
\hline \multicolumn{7}{|c|}{$\begin{array}{c}\text { base, } 0.025 \mathrm{mmol} \text { or } 0.05 \mathrm{mmol} \text {; solvent, } 1 \mathrm{~mL} ;{ }^{a} \text { Determined by } 400 \mathrm{MHz} \text { NMR with } \\
\text { 1,3,5-trimethoxybenzene as the internal standard. }\end{array}$} \\
\hline
\end{tabular}

2.7 Synthesis of $N$-heterocycles from olefinic sulfonamides

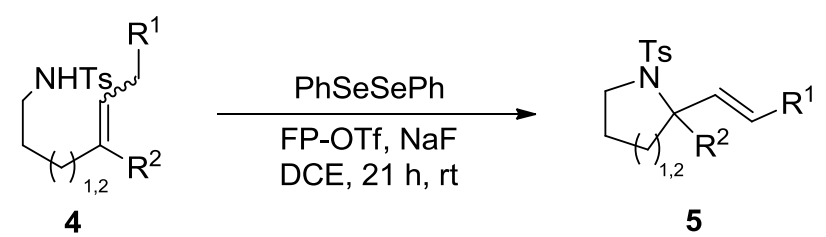

General procedure A for cyclization of olefinic sulfonamides $\mathbf{4 a - 4 f}$ :

A 4-mL vial equipped with a magnetic stir bar was charged with FP-OTf (29.7 mg, $0.12 \mathrm{mmol}, 1.2$ euqiv), $\mathrm{NaF}$ (4.2 mg, $0.1 \mathrm{mmol}, 1$ equiv) or $\mathrm{NaHCO}_{3}(6.7 \mathrm{mg}, 0.08$ mmol, 0.8 equiv), $\mathrm{PhSeSePh}(3.1 \mathrm{mg}, 0.01 \mathrm{mmol}, 10 \mathrm{~mol} \%$ ) and olefinic sulfonamides $4(0.1 \mathrm{mmol})$. Then dried DCE $(2 \mathrm{~mL})$ was added, and the vial was capped. After stirred at room temperature for $21 \mathrm{~h}$, the resulting mixture was concentrated in vacuo. The residue was purified by silica gel column chromatography to afford the corresponding tetrahydropyrroles or piperidines $\mathbf{5}$.<smiles></smiles>

(E)-2-Phenyl-2-(3-phenylprop-1-en-1-yl)-1-tosylpyrrolidine (5a): Prepared according to general procedure A. Flash column chromatography (eluent: $\mathrm{PE} / \mathrm{DCM}=$ $1: 2, \mathrm{v} / \mathrm{v})$ to afford $\mathbf{5 a}(30.0 \mathrm{mg}, 72 \%)$ as a white oil. ${ }^{1} \mathrm{H}$ NMR $\left(400 \mathrm{MHz}, \mathrm{CDCl}_{3}\right) \delta$ 
$7.57(\mathrm{~d}, J=8.2 \mathrm{~Hz}, 2 \mathrm{H}), 7.45(\mathrm{~d}, J=7.5 \mathrm{~Hz}, 2 \mathrm{H}), 7.36-7.28(\mathrm{~m}, 4 \mathrm{H}), 7.25(\mathrm{t}, J=7.0$ $\mathrm{Hz}, 2 \mathrm{H}), 7.22-7.15(\mathrm{~m}, 4 \mathrm{H}), 6.18(\mathrm{~d}, J=15.7 \mathrm{~Hz}, 1 \mathrm{H}), 5.85(\mathrm{dt}, J=15.6,7.0 \mathrm{~Hz}$, $1 \mathrm{H}), 3.77(\mathrm{td}, J=8.4,4.3 \mathrm{~Hz}, 1 \mathrm{H}), 3.56-3.47(\mathrm{~m}, 2 \mathrm{H}), 3.41(\mathrm{dd}, J=15.3,7.0 \mathrm{~Hz}$, $1 \mathrm{H}), 2.40(\mathrm{~s}, 3 \mathrm{H}), 2.28-2.19(\mathrm{~m}, 1 \mathrm{H}), 2.15-2.07(\mathrm{~m}, 1 \mathrm{H}), 1.86-1.68(\mathrm{~m}, 2 \mathrm{H}) .{ }^{13} \mathrm{C}$ NMR $\left(101 \mathrm{MHz}, \mathrm{CDCl}_{3}\right) \delta 144.4,142.6,140.1,138.6,133.3,130.6,129.2,128.7$, 128.6, 128.0, 127.3, 127.1, 127.0, 126.3, 73.7, 49.8, 42.0, 39.0, 22.2, 21.6. HR-ESI-MS m/z calcd for $\mathrm{C}_{26} \mathrm{H}_{27} \mathrm{NO}_{2} \mathrm{~S}[\mathrm{M}+\mathrm{H}]^{+}$: 418.1835, found: 418.1836.<smiles></smiles>

(E)-2-(But-1-en-1-yl)-2-(p-tolyl)-1-tosylpyrrolidine (5b): Prepared according to general procedure A. Flash column chromatography (eluent: $\mathrm{PE} / \mathrm{DCM}=2: 3$, v/v) to afford $\mathbf{5 b}(20.1 \mathrm{mg}, 54 \%)$ as a colorless oil. ${ }^{1} \mathrm{H}$ NMR $\left(400 \mathrm{MHz}, \mathrm{CDCl}_{3}\right) \delta 7.58(\mathrm{~d}, J$ $=8.3 \mathrm{~Hz}, 2 \mathrm{H}), 7.31(\mathrm{~d}, J=8.2 \mathrm{~Hz}, 2 \mathrm{H}), 7.21(\mathrm{~d}, J=8.0 \mathrm{~Hz}, 2 \mathrm{H}), 7.11(\mathrm{~d}, J=8.0 \mathrm{~Hz}$, $2 \mathrm{H}), 5.94(\mathrm{~d}, J=15.7 \mathrm{~Hz}, 1 \mathrm{H}), 5.66(\mathrm{dt}, J=15.7,6.4 \mathrm{~Hz}, 1 \mathrm{H}), 3.80-3.72(\mathrm{~m}, 1 \mathrm{H})$, $3.55-3.46(\mathrm{~m}, 1 \mathrm{H}), 2.40(\mathrm{~s}, 3 \mathrm{H}), 2.33(\mathrm{~s}, 3 \mathrm{H}), 2.24-2.14(\mathrm{~m}, 1 \mathrm{H}), 2.13-2.03(\mathrm{~m}$, $3 \mathrm{H}), 1.84-1.68(\mathrm{~m}, 2 \mathrm{H}), 0.99(\mathrm{t}, J=7.5 \mathrm{~Hz}, 3 \mathrm{H}) .{ }^{13} \mathrm{C} \mathrm{NMR}\left(101 \mathrm{MHz}, \mathrm{CDCl}_{3}\right) \delta$ 142.5, 141.8, 139.0, 136.4, 133.8, 131.1, 129.1, 128.7, 127.4, 127.1, 73.5, 50.0, 42.1, 25.7, 22.3, 21.6, 21.1, 13.5. HR-ESI-MS m/z calcd for $\mathrm{C}_{22} \mathrm{H}_{27} \mathrm{NO}_{2} \mathrm{~S}[\mathrm{M}+\mathrm{H}]^{+}$: 370.1835 , found: 370.1837 .<smiles>CC/C=C\C1(c2ccc(Cl)cc2)CCCN1[13CH3]</smiles>

(E)-2-(But-1-en-1-yl)-2-(p-tolyl)-1-tosylpyrrolidine (5c): Prepared according to general procedure A. Flash column chromatography (eluent: $\mathrm{PE} / \mathrm{DCM}=1: 2$, v/v) to afford 5c $(31.7 \mathrm{mg}, 81 \%)$ as a colorless oil. ${ }^{1} \mathrm{H}$ NMR $\left(400 \mathrm{MHz}, \mathrm{CDCl}_{3}\right) \delta 7.58(\mathrm{~d}, J=$ $8.3 \mathrm{~Hz}, 2 \mathrm{H}), 7.36(\mathrm{~d}, J=8.6 \mathrm{~Hz}, 2 \mathrm{H}), 7.26(\mathrm{~d}, J=8.7 \mathrm{~Hz}, 2 \mathrm{H}), 7.22(\mathrm{~d}, J=8.1 \mathrm{~Hz}$, $2 \mathrm{H}), 5.90(\mathrm{~d}, J=15.8 \mathrm{~Hz}, 1 \mathrm{H}), 5.67(\mathrm{dt}, J=15.7,6.3 \mathrm{~Hz}, 1 \mathrm{H}), 3.80-3.73(\mathrm{~m}, 1 \mathrm{H})$, $3.55-3.46(\mathrm{~m}, 1 \mathrm{H}), 2.41(\mathrm{~s}, 3 \mathrm{H}), 2.26-2.16(\mathrm{~m}, 1 \mathrm{H}), 2.15-1.98(\mathrm{~m}, 3 \mathrm{H}), 1.86-$ $1.66(\mathrm{~m}, 2 \mathrm{H}), 0.99(\mathrm{t}, J=7.5 \mathrm{~Hz}, 3 \mathrm{H}) .{ }^{13} \mathrm{C} \mathrm{NMR}\left(101 \mathrm{MHz}, \mathrm{CDCl}_{3}\right) \delta 143.5,142.8$, 138.7, 134.5, 132.7, 130.4, 129.2, 128.6, 128.0, 127.3, 73.1, 50.0, 42.2, 25.7, 22.2, 21.6, 13.4. HR-ESI-MS $\mathrm{m} / \mathrm{z}$ calcd for $\mathrm{C}_{21} \mathrm{H}_{24} \mathrm{ClNO}_{2} \mathrm{~S}[\mathrm{M}+\mathrm{H}]^{+}: 390.1289$, found: 390.1290 .

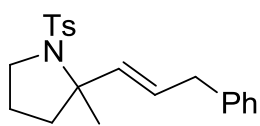

(E)-2-Methyl-2-(3-phenylprop-1-en-1-yl)-1-tosylpyrrolidine $\quad(5 d): \quad$ Prepared according to general procedure A. Flash column chromatography (eluent: PE/EtOAc $=15: 1, \mathrm{v} / \mathrm{v})$ to afford $\mathbf{5 d}(33.1 \mathrm{mg}, 93 \%)$ as a colorless oil. ${ }^{1} \mathrm{H}$ NMR $(400 \mathrm{MHz}$, $\left.\mathrm{CDCl}_{3}\right) \delta 7.69(\mathrm{~d}, J=8.2 \mathrm{~Hz}, 2 \mathrm{H}), 7.30(\mathrm{t}, J=7.4 \mathrm{~Hz}, 2 \mathrm{H}), 7.22(\mathrm{t}, J=7.6 \mathrm{~Hz}, 3 \mathrm{H})$, 
$7.18(\mathrm{~d}, J=7.2 \mathrm{~Hz}, 2 \mathrm{H}), 5.76(\mathrm{dt}, J=15.4,6.8 \mathrm{~Hz}, 1 \mathrm{H}), 5.55(\mathrm{~d}, J=15.5 \mathrm{~Hz}, 1 \mathrm{H})$, $3.54-3.39(\mathrm{~m}, 2 \mathrm{H}), 3.39-3.27(\mathrm{~m}, 2 \mathrm{H}), 2.41(\mathrm{~s}, 3 \mathrm{H}), 1.94-1.72(\mathrm{~m}, 4 \mathrm{H}), 1.58(\mathrm{~s}$, $3 \mathrm{H}) .{ }^{13} \mathrm{C} \mathrm{NMR}\left(101 \mathrm{MHz}, \mathrm{CDCl}_{3}\right) \delta 142.6,140.4,138.7,136.2,129.3,128.6,128.5$, 127.6, 127.4, 126.1, 67.4, 49.4, 42.2, 38.7, 25.8, 22.6, 21.6. HR-ESI-MS m/z calcd for $\mathrm{C}_{21} \mathrm{H}_{25} \mathrm{NO}_{2} \mathrm{~S}[\mathrm{M}+\mathrm{H}]^{+}: 356.1679$, found: 356.1678 .<smiles>C=CC1(c2ccccc2)CCC[N]1</smiles>

2-Phenyl-1-tosyl-2-vinylpyrrolidine (5e): Prepared according to general procedure A. Flash column chromatography (eluent: PE/EtOAc $=15: 1$, v/v) to afford 5e $(25.0$ $\mathrm{mg}, 76 \%)$ as a colorless oil. ${ }^{1} \mathrm{H} \mathrm{NMR}\left(400 \mathrm{MHz}, \mathrm{CDCl}_{3}\right) \delta 7.52(\mathrm{~d}, J=8.2 \mathrm{~Hz}, 2 \mathrm{H})$, $7.40(\mathrm{~d}, J=7.3 \mathrm{~Hz}, 2 \mathrm{H}), 7.30(\mathrm{t}, J=7.3 \mathrm{~Hz}, 2 \mathrm{H}), 7.25-7.17(\mathrm{~m}, 3 \mathrm{H}), 6.43(\mathrm{dd}, J=$ $17.3,10.8 \mathrm{~Hz}, 1 \mathrm{H}), 5.35$ (dd, $J=14.1,6.4 \mathrm{~Hz}, 2 \mathrm{H}), 3.74-3.66(\mathrm{~m}, 1 \mathrm{H}), 3.60-3.52$ (m, 1H), $2.40(\mathrm{~s}, 3 \mathrm{H}), 2.29-2.20(\mathrm{~m}, 1 \mathrm{H}), 2.18-2.08(\mathrm{~m}, 1 \mathrm{H}), 1.92-1.73(\mathrm{~m}, 2 \mathrm{H})$. ${ }^{13} \mathrm{C}$ NMR $\left(101 \mathrm{MHz}, \mathrm{CDCl}_{3}\right) \delta 143.5,142.7,139.8,138.3,129.2,128.0,127.3,127.3$, 127.1, 116.3, 74.1, 49.8, 41.7, 22.3, 21.6. HR-ESI-MS m/z calcd for $\mathrm{C}_{19} \mathrm{H}_{21} \mathrm{NO}_{2} \mathrm{~S}$ $[\mathrm{M}+\mathrm{H}]^{+}:$328.1366, found: 328.1365 .<smiles>[N]C1CCCCC1/C=C/Cc1ccccc1</smiles>

(E)-2-(But-1-en-1-yl)-2-(p-tolyl)-1-tosylpyrrolidine (5f): Prepared according to general procedure A. Flash column chromatography (eluent: PE/EtOAc $=30: 1, \mathrm{v} / \mathrm{v}$ ) to afford $\mathbf{5 f}(25.1 \mathrm{mg}, 71 \%, Z / E=1: 13)$ as a colorless oil. $1 \mathrm{H} \mathrm{NMR} \mathrm{(400} \mathrm{MHz,}$ CDCl3) $\delta 7.64(\mathrm{~d}, J=8.2 \mathrm{~Hz}, 2 \mathrm{H}), 7.29(\mathrm{~d}, J=7.1 \mathrm{~Hz}, 2 \mathrm{H}), 7.20(\mathrm{~d}, J=8.1 \mathrm{~Hz}, 3 \mathrm{H})$, $7.09(\mathrm{~d}, J=7.2 \mathrm{~Hz}, 2 \mathrm{H}), 5.70(\mathrm{dt}, J=13.7,6.7 \mathrm{~Hz}, 1 \mathrm{H}), 5.41(\mathrm{dd}, J=15.5,6.0 \mathrm{~Hz}$, $1 \mathrm{H}), 4.59(\mathrm{~s}, 1 \mathrm{H}), 3.67(\mathrm{~d}, J=13.2 \mathrm{~Hz}, 1 \mathrm{H}), 3.26(\mathrm{~d}, J=6.7 \mathrm{~Hz}, 2 \mathrm{H}), 3.01-2.90(\mathrm{~m}$, $1 \mathrm{H}), 2.40(\mathrm{~s}, 3 \mathrm{H}), 1.74-1.36(\mathrm{~m}, 6 \mathrm{H}) .{ }^{13} \mathrm{C} \mathrm{NMR}\left(101 \mathrm{MHz}, \mathrm{CDCl}_{3}\right) \delta 142.8,140.0$, $137.9,132.0,129.5,128.6,128.5,128.2,127.5,126.2,54.7,41.9,38.8,30.5,25.3$, 21.6, 19.3. HR-ESI-MS $\mathrm{m} / \mathrm{z}$ calcd for $\mathrm{C}_{21} \mathrm{H}_{25} \mathrm{NO}_{2} \mathrm{~S}[\mathrm{M}+\mathrm{H}]^{+}$: 356.1679 , found: 356.1677 .

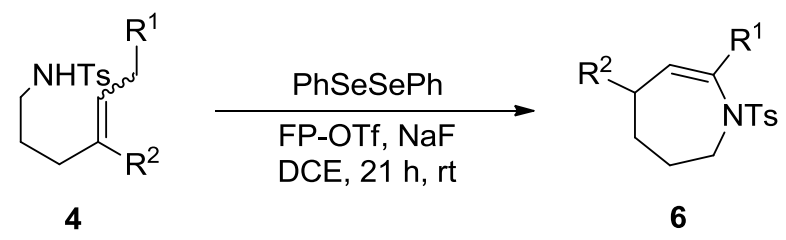

General procedure B for cyclization of olefinic sulfonamides $\mathbf{4 a - 4 c}$ :

A 4-mL vial equipped with a magnetic stir bar was charged with FP-OTf (29.7 mg, $0.12 \mathrm{mmol}, 1.2$ euqiv), $\mathrm{NaF}$ ( $2.1 \mathrm{mg}, 0.05 \mathrm{mmol}, 0.5$ equiv), $\mathrm{PhSeSePh}$ ( $3.1 \mathrm{mg}, 0.01$ mmol, $10 \mathrm{~mol} \%)$ and olefinic sulfonamides $4(0.1 \mathrm{mmol})$. Then dried DCE $(2 \mathrm{~mL})$ was added, and the vial was capped. After stirred at room temperature for $21 \mathrm{~h}$, the resulting mixture was concentrated in vacuo. The residue was purified by silica gel column chromatography to afford the corresponding azepines 6. 
<smiles>[NH3+]C(Br)C1C=C(c2ccccc2)CCC1</smiles>

7-Benzyl-5-phenyl-1-tosyl-2,3,4,7-tetrahydro-1H-azepine (6a): Prepared according to general procedure B. Flash column chromatography (eluent: $\mathrm{PE} / \mathrm{DCM}=2: 3, \mathrm{v} / \mathrm{v}$ ) to afford $6 \mathbf{a}(26.0 \mathrm{mg}, 62 \%)$ as a white solid. ${ }^{1} \mathrm{H} \mathrm{NMR}\left(400 \mathrm{MHz}, \mathrm{CDCl}_{3}\right) \delta 7.66(\mathrm{~d}, J$ $=8.3 \mathrm{~Hz}, 2 \mathrm{H}), 7.32-7.18(\mathrm{~m}, 10 \mathrm{H}), 7.14(\mathrm{~d}, J=6.8 \mathrm{~Hz}, 2 \mathrm{H}), 5.62(\mathrm{dd}, J=5.1,1.5$ $\mathrm{Hz}, 1 \mathrm{H}), 5.08(\mathrm{dd}, J=13.6,6.5 \mathrm{~Hz}, 1 \mathrm{H}), 3.86(\mathrm{dt}, J=14.5,7.2 \mathrm{~Hz}, 1 \mathrm{H}), 3.38(\mathrm{dt}, J=$ 14.4, $6.1 \mathrm{~Hz}, 1 \mathrm{H}), 3.02-2.90(\mathrm{~m}, 2 \mathrm{H}), 2.60-2.43(\mathrm{~m}, 2 \mathrm{H}), 2.40(\mathrm{~s}, 3 \mathrm{H}), 2.07-1.96$ $(\mathrm{m}, 1 \mathrm{H}), 1.95-1.83(\mathrm{~m}, 1 \mathrm{H}) .{ }^{13} \mathrm{C} \mathrm{NMR}\left(101 \mathrm{MHz}, \mathrm{CDCl}_{3}\right) \delta 144.3,143.0,142.4$, 138.4, 138.0, 129.7, 129.4, 128.7, 128.3, 127.5, 127.2, 127.0, 126.7, 126.0, 58.6, 43.9, 40.5, 29.2, 27.1, 21.6. HR-ESI-MS m/z calcd for $\mathrm{C}_{26} \mathrm{H}_{27} \mathrm{NO}_{2} \mathrm{~S}[\mathrm{M}+\mathrm{H}]^{+}:$418.1835, found: 418.1837 .<smiles>CCC1C=C(CC(C)(C)C)CCC[NH2+]1</smiles>

5-(4-Chlorophenyl)-7-ethyl-1-tosyl-2,3,4,7-tetrahydro-1H-azepine (6b): Prepared according to general procedure $\mathrm{B}$. Flash column chromatography (eluent: $\mathrm{PE} / \mathrm{DCM}=$ $2: 3, \mathrm{v} / \mathrm{v})$ to afford $\mathbf{6 b}(32.1 \mathrm{mg}, 82 \%)$ as a colorless oil. ${ }^{1} \mathrm{H} \mathrm{NMR}\left(400 \mathrm{MHz}, \mathrm{CDCl}_{3}\right) \delta$ $7.72(\mathrm{~d}, J=8.2 \mathrm{~Hz}, 2 \mathrm{H}), 7.25-7.20(\mathrm{~m}, 4 \mathrm{H}), 7.06(\mathrm{~d}, J=8.5 \mathrm{~Hz}, 2 \mathrm{H}), 5.71(\mathrm{dd}, J=$ 5.7, $1.3 \mathrm{~Hz}, 1 \mathrm{H}), 4.67(\mathrm{dd}, J=13.8,6.8 \mathrm{~Hz}, 1 \mathrm{H}), 3.85(\mathrm{dt}, J=14.8,7.4 \mathrm{~Hz}, 1 \mathrm{H}), 3.31$ (dt, $J=14.3,5.4 \mathrm{~Hz}, 1 \mathrm{H}), 2.40(\mathrm{~s}, 3 \mathrm{H}), 2.38-2.25(\mathrm{~m}, 2 \mathrm{H}), 1.94-1.77(\mathrm{~m}, 2 \mathrm{H}), 1.77$ $-1.63(\mathrm{~m}, 2 \mathrm{H}), 0.91(\mathrm{t}, J=7.4 \mathrm{~Hz}, 3 \mathrm{H}) .{ }^{13} \mathrm{C} \mathrm{NMR}\left(101 \mathrm{MHz}, \mathrm{CDCl}_{3}\right) \delta 143.0,143.0$, 141.6, 138.6, 132.7, 129.5, 129.4, 128.3, 127.4, 127.2, 58.4, 43.7, 29.8, 27.4, 26.5, 21.6, 11.4. HR-ESI-MS $\mathrm{m} / \mathrm{z}$ calcd for $\mathrm{C}_{21} \mathrm{H}_{24} \mathrm{ClNO}_{2} \mathrm{~S}[\mathrm{M}+\mathrm{H}]^{+}: 390.1289$, found: 390.1291 .<smiles>CCC1C=C(Cl)CCC[NH2+]1</smiles>

7-Benzyl-5-phenyl-1-tosyl-2,3,4,7-tetrahydro-1H-azepine (6c): Prepared according to general procedure B. Flash column chromatography (eluent: $\mathrm{PE} / \mathrm{DCM}=2: 3$, v/v) to afford $6 \mathbf{c}(16.6 \mathrm{mg}, 45 \%)$ as a colorless oil. ${ }^{1} \mathrm{H}$ NMR $\left(400 \mathrm{MHz}, \mathrm{CDCl}_{3}\right) \delta 7.74(\mathrm{~d}$, $J=8.2 \mathrm{~Hz}, 2 \mathrm{H}), 7.24(\mathrm{~d}, J=8.1 \mathrm{~Hz}, 2 \mathrm{H}), 7.08(\mathrm{~d}, J=8.1 \mathrm{~Hz}, 2 \mathrm{H}), 7.04(\mathrm{~d}, J=8.2 \mathrm{~Hz}$, $2 \mathrm{H}), 5.70(\mathrm{dd}, J=5.7,1.3 \mathrm{~Hz}, 1 \mathrm{H}), 4.69(\mathrm{dd}, J=13.6,6.8 \mathrm{~Hz}, 1 \mathrm{H}), 3.84(\mathrm{dt}, J=14.4$, $7.3 \mathrm{~Hz}, 1 \mathrm{H}), 3.33(\mathrm{dt}, J=14.3,5.5 \mathrm{~Hz}, 1 \mathrm{H}), 2.40(\mathrm{~s}, 3 \mathrm{H}), 2.45-2.21(\mathrm{~m}, 2 \mathrm{H}), 2.32(\mathrm{~s}$, $3 \mathrm{H}), 1.92-1.63(\mathrm{~m}, 4 \mathrm{H}), 0.93(\mathrm{t}, J=7.4 \mathrm{~Hz}, 3 \mathrm{H}) .{ }^{13} \mathrm{C} \mathrm{NMR}\left(101 \mathrm{MHz}, \mathrm{CDCl}_{3}\right) \delta$ 142.9, 142.4, 141.8, 138.7, 136.7, 129.5, 128.9, 128.0, 127.2, 126.0, 58.4, 43.8, 29.8, 27.3, 26.7, 21.6, 21.1, 11.3. HR-ESI-MS m/z calcd for $\mathrm{C}_{22} \mathrm{H}_{27} \mathrm{NO}_{2} \mathrm{~S}[\mathrm{M}+\mathrm{H}]^{+}$: 370.1835 , found: 370.1838 . 


\section{Selective cyclization of olefinic alcohol 7}

\subsection{Synthesis of (2E,7E)-5-(hydroxymethyl)-5-phenylnona-2,7-dien-1-yl propyl carbonate (7)}

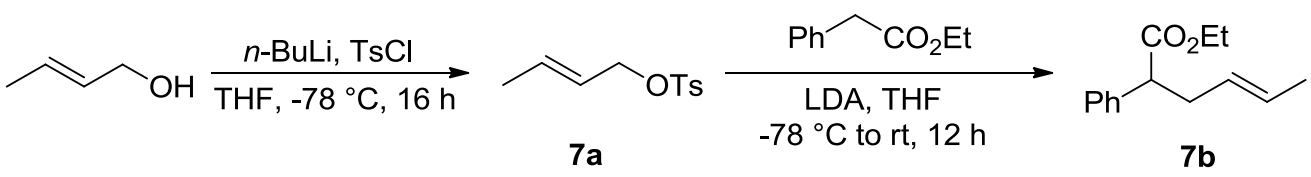

Procedure for synthesis of (E)-ethyl 2-phenylhex-4-enoate (7b):

This is a modified literature procedure. ${ }^{7}$ An oven-dried $100 \mathrm{~mL}$ Schlenk flask equipped with a stir bar was evacuated and back-filled with nitrogen. Then crotonyl alcohol $(853 \mu \mathrm{L}, 10 \mathrm{mmol})$ and anhydrous THF $(35 \mathrm{~mL})$ were subsequently added via syringe. The colorless solution was cooled to $-78{ }^{\circ} \mathrm{C}$ and $n$-BuLi $(2.5 \mathrm{M}$ in hexane) (4.2 $\mathrm{mL}, 10.5 \mathrm{mmol}$ ) was added dropwise via syringe over $5 \mathrm{~min}$, after which a solution of tosyl chloride (10 mmol, $1.91 \mathrm{~g})$ in anhydrous THF $(5 \mathrm{~mL})$ was added to the solution at $-78{ }^{\circ} \mathrm{C}$ over $10 \mathrm{~min}$. After stirred at $-78{ }^{\circ} \mathrm{C}$ for $16 \mathrm{~h}$, the resulting mixture was diluted with cold petroleum ether $(40 \mathrm{~mL})$ at $-78{ }^{\circ} \mathrm{C}$. The still cold solution was washed with saturated aqueous $\mathrm{NaHCO}_{3}$ solution $(40 \mathrm{~mL} \times 2)$, and then the combined aqueous phase was extracted with petroleum ether $(50 \mathrm{~mL})$. The organic phase was combined and dried over $\mathrm{K}_{2} \mathrm{CO}_{3}$ at $0{ }^{\circ} \mathrm{C}$. After filtration, the solution was concentrated in vacuo to afford $\mathbf{7 a}$ in quantitative yield, as a pale yellow oil.

An oven-dried $50 \mathrm{~mL}$ Schlenk flask equipped with a stir bar was evacuated and back-filled with nitrogen. Then anhydrous THF was added via syringe and cooled to $-78^{\circ} \mathrm{C}$. To the solution was added LDA (2.0 M in THF/n-heptane/ethylenzene) (5 mL, $10 \mathrm{mmol}$ ) dropwise via syringe, after which a solution of ethyl 2-phenylacetate (1.64 $\mathrm{g}, 9 \mathrm{mmol})$ in anhydrous THF was added to the solution at $-78{ }^{\circ} \mathrm{C}$ over $10 \mathrm{~min}$. The resulting orange solution was stirred at $-78^{\circ} \mathrm{C}$ for $1 \mathrm{~h}$. Then a solution of $7 \mathbf{a}(9 \mathrm{mmol})$ in anhydrous THF $(3 \mathrm{~mL})$ was added via syringe at $-78^{\circ} \mathrm{C}$ over $10 \mathrm{~min}$. The solution was warmed to room temperature gradually and stirred for $12 \mathrm{~h}$. The resulting heterogeneous mixture was quenched with $\mathrm{H}_{2} \mathrm{O}(40 \mathrm{~mL})$ and extracted with DCM (40 $\mathrm{mL} \times 3$ ). The organic extracts were dried over anhydrous $\mathrm{Na}_{2} \mathrm{SO}_{4}$, filtered and concentrated in vacuo. The residue was purified by silica gel column chromatography (eluent: PE/EtOAc $=60: 1, \mathrm{v} / \mathrm{v})$ to afford $7 \mathbf{b}$ as a yellow oil $(1.87 \mathrm{~g}, 95 \%$ yield $) .{ }^{1} \mathrm{H}$ NMR (400 MHz, $\left.\mathrm{CDCl}_{3}\right) \delta 7.35-7.28(\mathrm{~m}, 5 \mathrm{H}), 5.50(\mathrm{dq}, J=12.7,6.3 \mathrm{~Hz}, 1 \mathrm{H}), 5.40$ - $5.29(\mathrm{~m}, 1 \mathrm{H}), 4.18-4.06(\mathrm{~m}, 2 \mathrm{H}), 3.62-3.53(\mathrm{~m}, 1 \mathrm{H}), 2.80-2.69(\mathrm{~m}, 1 \mathrm{H}), 2.48-$ $2.36(\mathrm{~m}, 1 \mathrm{H}), 1.61(\mathrm{dd}, J=6.3,1.2 \mathrm{~Hz}, 3 \mathrm{H}), 1.20(\mathrm{t}, J=7.1 \mathrm{~Hz}, 3 \mathrm{H})$.

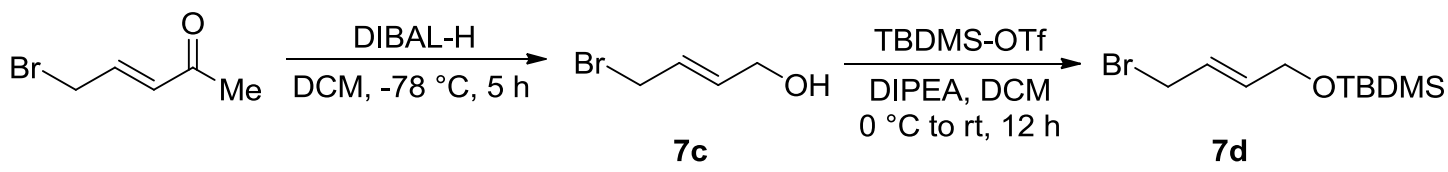

Procedure for synthesis of (E)-ethyl 2-phenylhex-4-enoate (7d): 
This is a modified literature procedure. ${ }^{8}$ An oven-dried $50 \mathrm{~mL}$ Schlenk flask equipped with a stir bar was evacuated and back-filled with nitrogen. Then methyl 4-bromocrotonate $(589 \mu \mathrm{L}, 5 \mathrm{mmol})$ in anhydrous DCM $(7.5 \mathrm{~mL})$ was added via syringe. The amber solution was cooled to $-78{ }^{\circ} \mathrm{C}$ and DIBAL-H (1.0 $\mathrm{M}$ in toluene) $(12.5 \mathrm{~mL}, 12.5 \mathrm{mmol})$ was added dropwise via syringe over $30 \mathrm{~min}$. After stirred at $-78{ }^{\circ} \mathrm{C}$ for $5 \mathrm{~h}$, the resulting solution was warmed to room temperature, and then quenched with aqueous $1 \mathrm{M} \mathrm{HCl}$ solution $(30 \mathrm{~mL})$ carefully. After stirred for additional $1 \mathrm{~h}$, the mixture was extracted with DCM $(30 \mathrm{~mL} \times 3)$. The organic extracts were dried over anhydrous $\mathrm{Na}_{2} \mathrm{SO}_{4}$, filtered and concentrated in vacuo. The crude 7c and DCM $(10 \mathrm{~mL})$ was added to an oven-dried $25 \mathrm{~mL}$ Schlenk flask under nitrogen. The solution was cooled to $0{ }^{\circ} \mathrm{C}$, then diisopropylethylamine $(826 \mu \mathrm{L}, 5 \mathrm{mmol})$ and TBDMS-OTf $(1.15 \mathrm{~mL}, 5 \mathrm{mmol})$ was added. The resulting mixture was warmed to room temperature gradually and stirred for $12 \mathrm{~h}$, after which the homogeneous mixture was concentrated in vacuo. The residue was purified by silica gel column chromatography (eluent: $\mathrm{PE}$ to $\mathrm{PE} / \mathrm{EtOAc}=60: 1, \mathrm{v} / \mathrm{v}$ ) to afford 7d as a pale yellow oil $\left(0.71 \mathrm{~g}, 53 \%\right.$ yield in two steps). ${ }^{1} \mathrm{H}$ NMR $\left(400 \mathrm{MHz}, \mathrm{CDCl}_{3}\right) \delta 5.93(\mathrm{dt}, J=15.1$, $7.2 \mathrm{~Hz}, 1 \mathrm{H}), 5.85(\mathrm{dt}, J=15.4,4.3 \mathrm{~Hz}, 1 \mathrm{H}), 4.20(\mathrm{~d}, J=4.0 \mathrm{~Hz}, 2 \mathrm{H}), 3.98(\mathrm{~d}, J=7.1$ $\mathrm{Hz}, 2 \mathrm{H}), 0.91(\mathrm{~s}, 12 \mathrm{H}), 0.07$ (s, 6H).
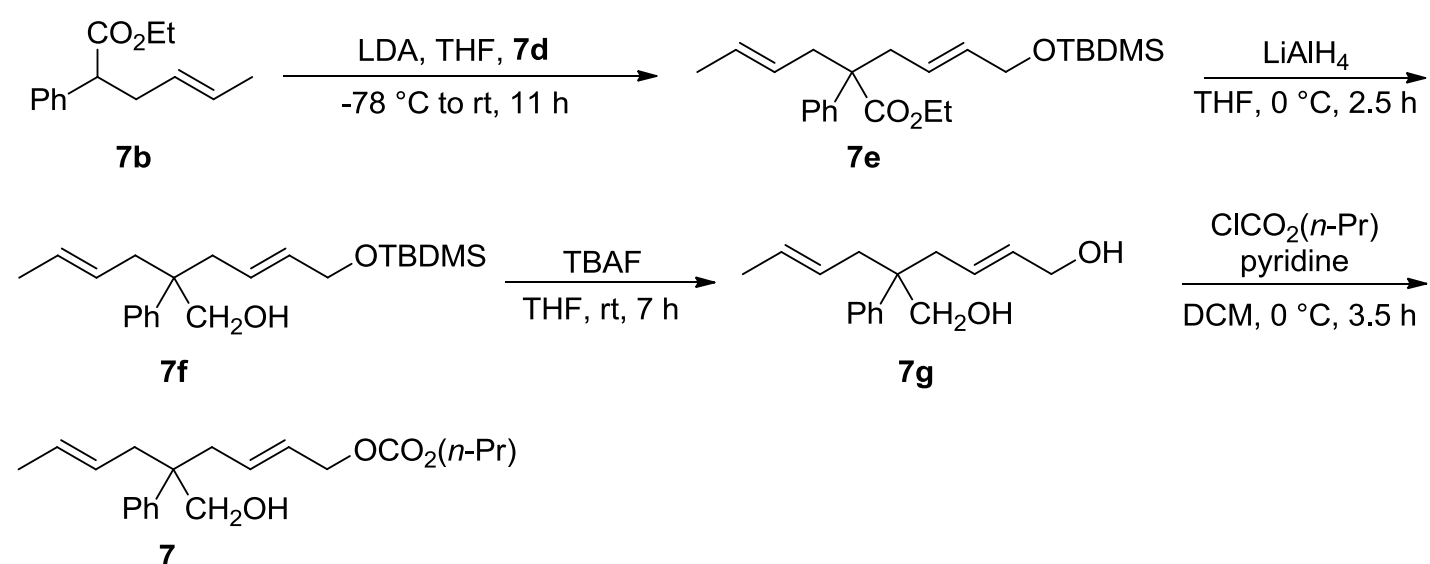

Procedure for synthesis of (2E,7E)-5-(hydroxymethyl)-5-phenylnona-2,7-dien-1-yl propyl carbonate (7):

The procedure for synthesis of $\mathbf{7 e}$ is similar to the synthesis of $\mathbf{7 b}$. Flash column chromatography (eluent: PE/EtOAc $=80: 1, \mathrm{v} / \mathrm{v})$ to afford $7 \mathrm{e}(0.72 \mathrm{~g}, 74 \%$ yield $)$ as a pale yellow oil. ${ }^{1} \mathrm{H}$ NMR $\left(400 \mathrm{MHz}, \mathrm{CDCl}_{3}\right) \delta 7.34-7.28(\mathrm{~m}, 3 \mathrm{H}), 7.25-7.19(\mathrm{~m}$, $2 \mathrm{H}), 5.55(\mathrm{dt}, J=15.4,5.1 \mathrm{~Hz}, 1 \mathrm{H}), 5.50-5.42(\mathrm{~m}, 1 \mathrm{H}), 5.40-5.30(\mathrm{~m}, 1 \mathrm{H}), 5.20-$ $5.10(\mathrm{~m}, 1 \mathrm{H}), 4.11(\mathrm{q}, J=7.0 \mathrm{~Hz}, 2 \mathrm{H}), 4.06(\mathrm{~d}, J=3.9 \mathrm{~Hz}, 1 \mathrm{H}), 2.81-2.60(\mathrm{~m}, 4 \mathrm{H})$, $1.61(\mathrm{dd}, J=6.3,1.0 \mathrm{~Hz}, 3 \mathrm{H}), 1.17(\mathrm{t}, J=7.1 \mathrm{~Hz}, 3 \mathrm{H}), 0.87(\mathrm{~s}, 9 \mathrm{H}), 0.02(\mathrm{~s}, 6 \mathrm{H})$.

An oven-dried $25 \mathrm{~mL}$ Schlenk flask equipped with a stir bar was charged with $\mathrm{LiAlH}_{4}$ (102 mg, $2.7 \mathrm{mmol}$ ). The flask was then evacuated and back-filled with nitrogen. Then anhydrous THF $(10 \mathrm{~mL})$ was added via syringe, after which the heterogeneous solution was cooled to $0{ }^{\circ} \mathrm{C}$. Then a solution of $7 \mathrm{e}(725 \mathrm{mg}, 1.8 \mathrm{mmol})$ in anhydrous THF ( $3 \mathrm{~mL}$ ) was added to the suspension at $0{ }^{\circ} \mathrm{C}$ over $5 \mathrm{~min}$. After stirred at $0{ }^{\circ} \mathrm{C}$ for 
$2.5 \mathrm{~h}$, the resulting heterogeneous mixture was quenched with $\mathrm{H}_{2} \mathrm{O}(200 \mu \mathrm{L})$ and aqueous $10 \% \mathrm{NaOH}$ solution $(200 \mu \mathrm{L})$ carefully. After stirred at room temperature for additional $2 \mathrm{~h}$, the resulting mixture was dried over anhydrous $\mathrm{Na}_{2} \mathrm{SO}_{4}$, filtered and concentrated in vacuo. The residue was purified by silica gel column chromatography (eluent: PE/EtOAc $=25: 1, \mathrm{v} / \mathrm{v})$ to afford $7 \mathbf{f}$ as a colorless oil $(0.43 \mathrm{~g}, 66 \%$ yield $) .{ }^{1} \mathrm{H}$ NMR $\left(400 \mathrm{MHz}, \mathrm{CDCl}_{3}\right) \delta 7.37-7.31(\mathrm{~m}, 4 \mathrm{H}), 7.25-7.19(\mathrm{~m}, 1 \mathrm{H}), 5.59(\mathrm{dt}, J=$ $15.4,5.0 \mathrm{~Hz}, 1 \mathrm{H}), 5.55-5.41(\mathrm{~m}, 2 \mathrm{H}), 5.30-5.22(\mathrm{~m}, 1 \mathrm{H}), 4.07(\mathrm{~d}, J=4.9 \mathrm{~Hz}, 2 \mathrm{H})$, $3.76(\mathrm{~s}, 2 \mathrm{H}), 2.55-2.34(\mathrm{~m}, 4 \mathrm{H}), 1.61(\mathrm{dd}, J=6.4,1.2 \mathrm{~Hz}, 3 \mathrm{H}), 0.86(\mathrm{~s}, 9 \mathrm{H}), 0.01(\mathrm{~s}$, $6 \mathrm{H})$.

A $25 \mathrm{~mL}$ round-bottom flask equipped with a stir bar was charged with $7 \mathbf{f}$ (433 $\mathrm{mg}$, $1.2 \mathrm{mmol})$ and anhydrous THF $(10 \mathrm{~mL})$. Then a solution of tetrabutylammonium fluoride $(471 \mathrm{mg}, 1.8 \mathrm{mmol})$ in anhydrous THF $(3 \mathrm{~mL})$ was added to the solution via syringe. After stirred at room temperature for $7 \mathrm{~h}$, the resulting mixture was diluted with $\mathrm{Et}_{2} \mathrm{O}(30 \mathrm{~mL})$ and washed with brine $(30 \mathrm{~mL} \times 2)$. The combined aqueous phase was extracted with $\mathrm{Et}_{2} \mathrm{O}(40 \mathrm{~mL} \times 2)$. The organic phase was combined, dried over anhydrous $\mathrm{Na}_{2} \mathrm{SO}_{4}$, filtered and concentrated in vacuo. The residue was purified by silica gel column chromatography (eluent: $\mathrm{PE} / \mathrm{EtOAc}=1: 1$ to $\mathrm{PE} / \mathrm{EtOAc}=1: 2 \mathrm{v} / \mathrm{v}$ ) to afford $\mathbf{7 g}$ as a pale yellow oil in quantitative yield. ${ }^{1} \mathrm{H}$ NMR $\left(400 \mathrm{MHz}, \mathrm{CDCl}_{3}\right) \delta$ $7.38-7.31(\mathrm{~m}, 4 \mathrm{H}), 7.25-7.20(\mathrm{~m}, 1 \mathrm{H}), 5.68(\mathrm{dt}, J=15.3,5.9 \mathrm{~Hz}, 1 \mathrm{H}), 5.57-5.44$ (m, 2H), $5.29-5.21(\mathrm{~m}, 1 \mathrm{H}), 4.02(\mathrm{~d}, J=5.4 \mathrm{~Hz}, 2 \mathrm{H}), 3.77(\mathrm{~s}, 2 \mathrm{H}), 2.54-2.34(\mathrm{~m}$, $4 \mathrm{H}), 1.62(\mathrm{dd}, J=6.4,1.4 \mathrm{~Hz}, 3 \mathrm{H})$.

This is a modified literature procedure. ${ }^{9}$ A $25 \mathrm{~mL}$ round-bottom flask equipped with a stir bar was charged with $7 \mathbf{g}(296 \mathrm{mg}, 1.2 \mathrm{mmol})$ and anhydrous DCM $(10 \mathrm{~mL})$. The colorless solution was cooled to $0{ }^{\circ} \mathrm{C}$, then pyridine $(193 \mu \mathrm{L}, 2.4 \mathrm{mmol})$ and $n$-propyl carbonochloridate $(146 \mu \mathrm{L}, 1.3 \mathrm{mmol})$ was added. The resulting mixture was warmed to room temperature gradually and stirred for $3.5 \mathrm{~h}$, after which the homogeneous mixture was concentrated in vacuo. The residue was purified by silica gel column chromatography (eluent: $\mathrm{PE} / \mathrm{EtOAc}=9: 1$ to $\mathrm{PE} / \mathrm{EtOAc}=3: 1, \mathrm{v} / \mathrm{v}$ ) to afford 7 as a colorless oil (0.21 g, 54\% yield). ${ }^{1} \mathrm{H}$ NMR $\left(400 \mathrm{MHz}, \mathrm{CDCl}_{3}\right) \delta 7.38-7.29(\mathrm{~m}, 4 \mathrm{H})$, $7.25-7.20(\mathrm{~m}, 1 \mathrm{H}), 5.68-5.57(\mathrm{~m}, 2 \mathrm{H}), 5.51(\mathrm{dq}, J=12.8,6.4 \mathrm{~Hz}, 1 \mathrm{H}), 5.24(\mathrm{dt}, J=$ 13.5, 6.4 Hz, 1H), $4.49(\mathrm{~d}, J=5.2 \mathrm{~Hz}, 2 \mathrm{H}), 4.07(\mathrm{t}, J=6.7 \mathrm{~Hz}, 2 \mathrm{H}), 3.76(\mathrm{~s}, 2 \mathrm{H}), 2.55$ $-2.33(\mathrm{~m}, 4 \mathrm{H}), 1.73-1.63(\mathrm{~m}, 2 \mathrm{H}), 1.61(\mathrm{dd}, J=6.4,1.2 \mathrm{~Hz}, 3 \mathrm{H}), 0.95(\mathrm{t}, J=7.4 \mathrm{~Hz}$, $3 \mathrm{H}) .{ }^{13} \mathrm{C} \mathrm{NMR}\left(101 \mathrm{MHz}, \mathrm{CDCl}_{3}\right) \delta 155.3,143.5,132.7,128.7,128.6,127.0,126.7$, 126.5, 126.5, 69.7, 68.3, 68.0, 46.2, 38.4, 38.2, 22.2, 18.2, 10.3. HR-ESI-MS m/z calcd for $\mathrm{C}_{20} \mathrm{H}_{28} \mathrm{O}_{4}[\mathrm{M}+\mathrm{Na}]^{+}: 355.1880$, found: 355.1876 .

\subsection{Cyclization of olefinic alcohol 7 through organoselenium catalysis}
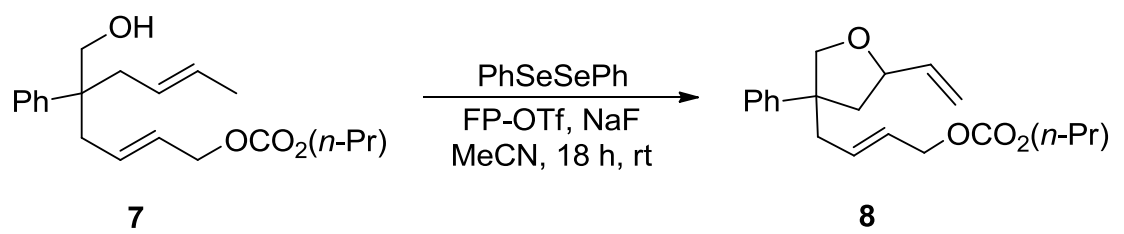
A 4-mL vial equipped with a magnetic stir bar was charged with FP-OTf (29.7 mg, $0.12 \mathrm{mmol}, 1.2$ euqiv), $\mathrm{NaF}$ (4.2 mg, $0.1 \mathrm{mmol}, 1$ equiv), $\mathrm{PhSeSePh}$ (1.6 mg, 0.005 mmol, $5 \mathrm{~mol} \%)$ and olefinic alcohol $7(33.2 \mathrm{mg}, 0.1 \mathrm{mmol})$. Then dried acetonitrile $(5 \mathrm{~mL})$ was added, and the vial was capped. After stirred at room temperature for $18 \mathrm{~h}$, the resulting mixture was concentrated in vacuo. The residue was purified by silica gel column chromatography (eluent: PE/EtOAc $=15: 1, \mathrm{v} / \mathrm{v}$ ) to afford (E)-4-(3-phenyl-5-vinyltetrahydrofuran-3-yl)but-2-en-1-yl propyl carbonate $\mathbf{8}$ as a colorless oil $(23.9 \mathrm{mg}, 72 \%$ yield, d.r. $=1: 1) .{ }^{1} \mathrm{H} \mathrm{NMR}\left(400 \mathrm{MHz}, \mathrm{CDCl}_{3}\right) \delta 7.36-$ $7.28(\mathrm{~m}, 2 \mathrm{H}), 7.22(\mathrm{t}, J=7.1 \mathrm{~Hz}, 1 \mathrm{H}), 7.17(\mathrm{~d}, J=7.1 \mathrm{~Hz}, 1 \mathrm{H}), 7.08(\mathrm{~d}, J=7.1 \mathrm{~Hz}$, $1 \mathrm{H}), 5.95$ (ddd, $J=17.0,10.3,6.5 \mathrm{~Hz}, 0.5 \mathrm{H}), 5.84$ (ddd, $J=17.0,10.2,6.7 \mathrm{~Hz}, 0.5 \mathrm{H}$ ), $5.62-5.40(\mathrm{~m}, 2 \mathrm{H}), 5.26(\mathrm{dd}, J=17.1,1.4 \mathrm{~Hz}, 1 \mathrm{H}), 5.11(\mathrm{dd}, J=17.3,10.3 \mathrm{~Hz}, 1 \mathrm{H})$, $4.59(\mathrm{dd}, J=16.0,6.6 \mathrm{~Hz}, 0.5 \mathrm{H}), 4.47(\mathrm{dd}, J=11.7,6.0 \mathrm{~Hz}, 2 \mathrm{H}), 4.38(\mathrm{dd}, J=14.4$, $7.6 \mathrm{~Hz}, 0.5 \mathrm{H}), 4.14-4.03(\mathrm{~m}, 3 \mathrm{H}), 3.97(\mathrm{dd}, J=21.9,8.4 \mathrm{~Hz}, 1 \mathrm{H}), 2.59-2.38(\mathrm{~m}$, $3 \mathrm{H}), 1.95(\mathrm{t}, J=6.3 \mathrm{~Hz}, 0.5 \mathrm{H}), 1.91(\mathrm{t}, J=6.2 \mathrm{~Hz}, 0.5 \mathrm{H}), 1.73-1.64(\mathrm{~m}, 2 \mathrm{H}), 0.95(\mathrm{t}$, $J=7.4 \mathrm{~Hz}, 3 \mathrm{H}) .{ }^{13} \mathrm{C} \mathrm{NMR}\left(101 \mathrm{MHz}, \mathrm{CDCl}_{3}\right) \delta 155.2,144.7,144.6,139.3,139.2$, 132.6, 132.3, 128.5, 128.4, 127.1, 126.9, 126.6, 126.5, 115.7, 115.4, 79.9, 79.7, 76.8, 76.5, 69.7, 69.7, 68.1, 51.8, 51.5, 44.1, 43.5, 42.8, 42.4, 22.1, 10.3. HR-ESI-MS m/z calcd for $\mathrm{C}_{20} \mathrm{H}_{26} \mathrm{O}_{4}[\mathrm{M}+\mathrm{Na}]^{+}: 353.1723$, found: 353.1719 .

\subsection{Cyclization of olefinic alcohol 7 through palladium catalysis}
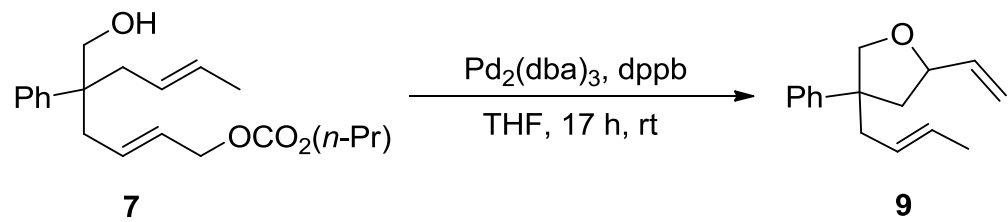

This is a modified literature procedure. ${ }^{10}$ An oven-dried $10 \mathrm{~mL}$ Schlenk flask equipped with a stir bar was charged with $\mathrm{Pd}_{2}(\mathrm{dba})_{3}(1.83 \mathrm{mg}, 0.002 \mathrm{mmol}, 2.5$ mol\%), dppb (1.71 mg, $0.004 \mathrm{mmol}, 5 \mathrm{~mol} \%)$. The flask was then evacuated and back-filled with nitrogen, then anhydrous THF $(1 \mathrm{~mL})$ was added via syringe. The resulting deep green solution was stirred at room temperature for $30 \mathrm{~min}$, after which a solution of 7 (26.6 mg, $0.08 \mathrm{mmol}$ ) in $1 \mathrm{~mL}$ anhydrous THF was added via syringe. After stirred at room temperature for $17 \mathrm{~h}$, the resulting mixture was concentrated in vacuo. The residue was purified by silica gel column chromatography (eluent: $\mathrm{PE} / \mathrm{EtOAc}=60: 1, \mathrm{v} / \mathrm{v})$ to afford $(E)$-4-(but-2-en-1-yl)-4-phenyl-2-vinyltetrahydrofuran 9 as a colorless oil $(22.6 \mathrm{mg}, 99 \%$ yield, d.r. $=5: 1) .{ }^{1} \mathrm{H} \mathrm{NMR}\left(400 \mathrm{MHz}, \mathrm{CDCl}_{3}\right)$ $\delta 7.31(\mathrm{t}, J=7.4 \mathrm{~Hz}, 2 \mathrm{H}), 7.23(\mathrm{~d}, J=7.4 \mathrm{~Hz}, 1 \mathrm{H}), 7.09(\mathrm{~d}, J=7.2 \mathrm{~Hz}, 2 \mathrm{H}), 5.85(\mathrm{ddd}$, $J=17.0,10.2,6.8 \mathrm{~Hz}, 1 \mathrm{H}), 5.48-5.34(\mathrm{~m}, 1 \mathrm{H}), 5.26(\mathrm{~d}, J=17.1 \mathrm{~Hz}, 1 \mathrm{H}), 5.16-$ $5.05(\mathrm{~m}, 2 \mathrm{H}), 4.59(\mathrm{dd}, J=15.8,6.8 \mathrm{~Hz}, 1 \mathrm{H}), 4.11(\mathrm{~d}, J=8.0 \mathrm{~Hz}, 1 \mathrm{H}), 3.94(\mathrm{~d}, J=$ $8.1 \mathrm{~Hz}, 1 \mathrm{H}), 2.50-2.35(\mathrm{~m}, 3 \mathrm{H}), 1.95-1.85(\mathrm{~m}, 1 \mathrm{H}), 1.60(\mathrm{~d}, J=6.8 \mathrm{~Hz}, 3 \mathrm{H}) .{ }^{13} \mathrm{C}$ NMR $\left(101 \mathrm{MHz}, \mathrm{CDCl}_{3}\right) \delta 145.4,139.5,128.8,128.3,127.0,126.8,126.3,115.3$, 79.8, 76.6, 52.0, 43.3, 42.5, 18.1. HR-ESI-MS m/z calcd for $\mathrm{C}_{16} \mathrm{H}_{21} \mathrm{O}[\mathrm{M}+\mathrm{H}]^{+}$: 229.1587, found: 229.1589 . 


\section{Mechanistic studies}

4.1 NMR studies of the equimolar mixture of PhSeSePh and FP-OTf

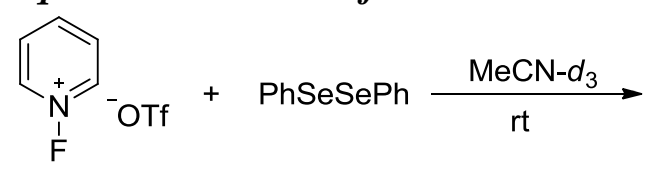

A dried NMR tube was charged with FP-OTf (29.7 mg, $0.15 \mathrm{mmol}), \mathrm{PhSeSePh}(1.6$ $\mathrm{mg}, 0.15 \mathrm{mmol}), \mathrm{MeCN}-d_{3}(500 \mu \mathrm{L})$, and $\mathrm{CFCl}_{3}(30 \mu \mathrm{L})$. The resulting heterogeneous mixture was analyzed by $500 \mathrm{MHz}$ NMR spectroscopy at room temperature for $24 \mathrm{~h}$. The ${ }^{1} \mathrm{H}$ NMR , ${ }^{19} \mathrm{~F}$ NMR, and ${ }^{77}$ Se NMR spectra of the mixture was collected every 1 $\mathrm{h}$ during the initial $9 \mathrm{~h}$ and furnished the last collection after $24 \mathrm{~h}$. (Number of scans: ${ }^{1} \mathrm{H}$ NMR(16), ${ }^{19} \mathrm{~F}$ NMR(16) and ${ }^{77} \operatorname{Se~NMR(3600))~}$

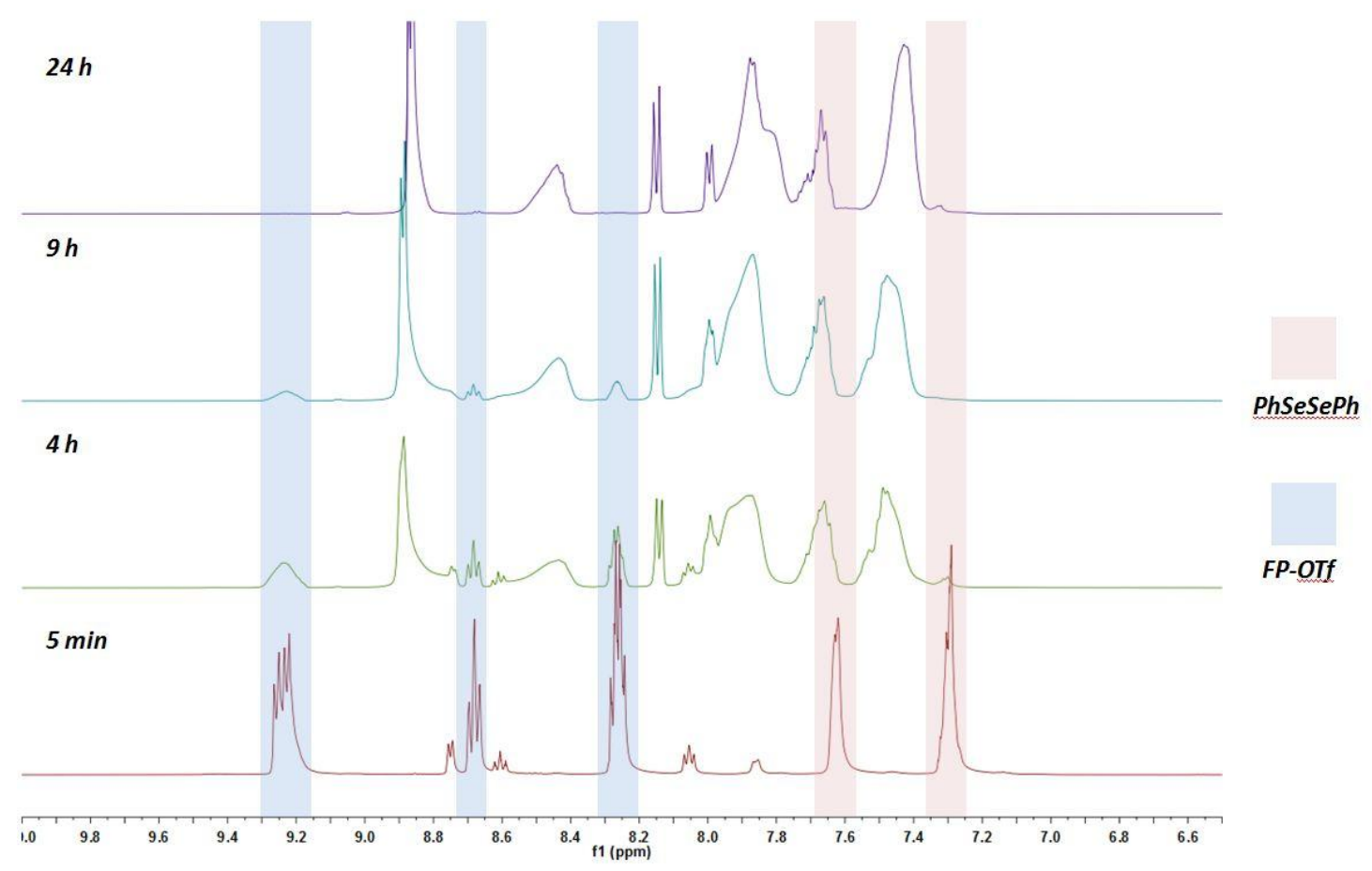

Scheme S1 Extract (6.50 to $10.0 \mathrm{ppm}$ ) of the ${ }^{1} \mathrm{H}$ NMR Spectra of Equimolar Mixture of PhSeSePh and FP-OTf in MeCN- $d_{3}$ 


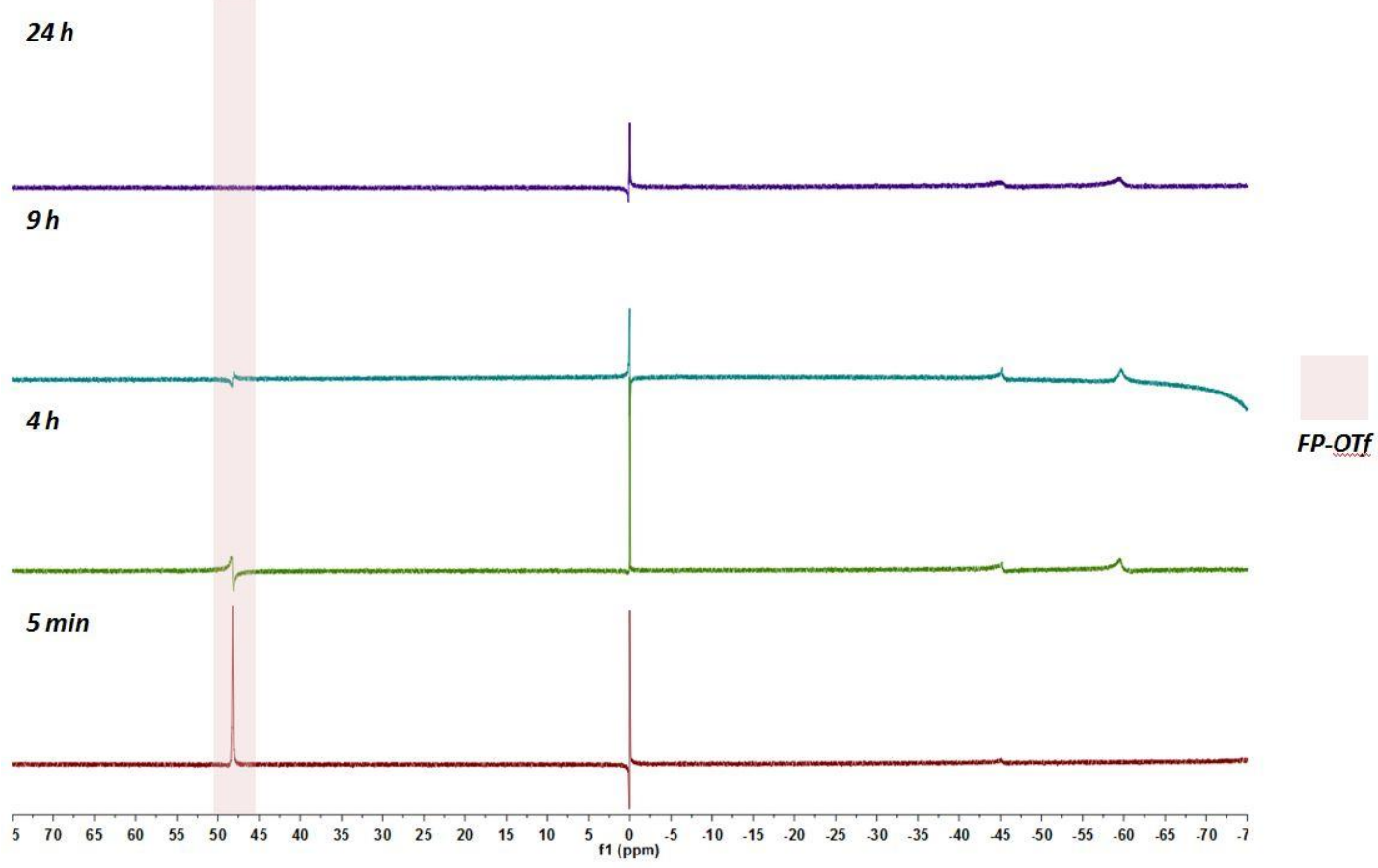

Scheme S2 Extract (75.0 to $\mathbf{- 7 5 . 0} \mathrm{ppm})$ of the ${ }^{19} \mathrm{~F}$ NMR Spectra of Equimolar Mixture of PhSeSePh and FP-OTf in MeCN- $d_{3}\left(\mathrm{CFCl}_{3}\right.$ was used as the internal standard)

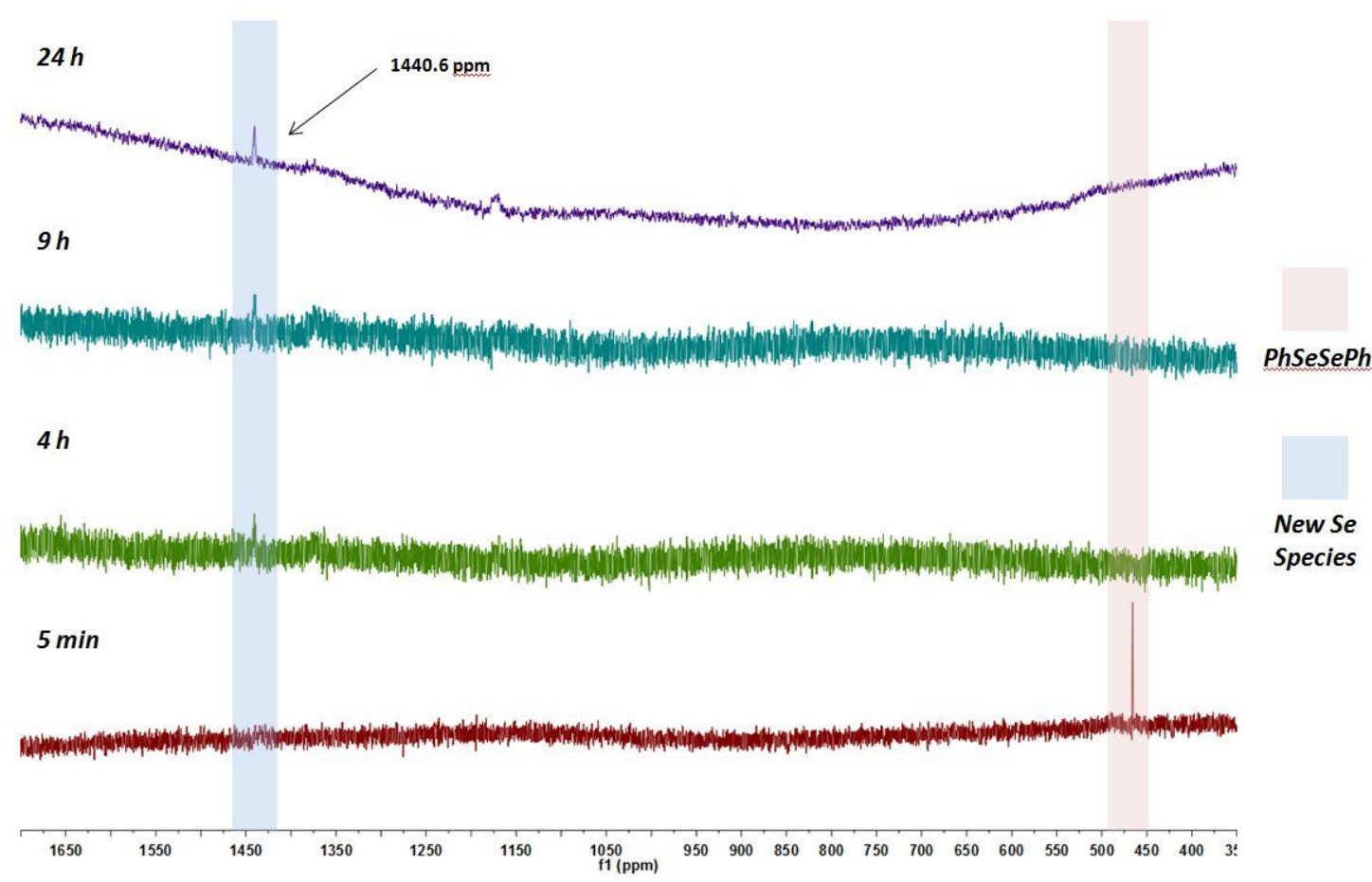

Scheme S3 Extract (1700 to 350.0 ppm) of the ${ }^{77}$ Se NMR Spectra of Equimolar Mixture of PhSeSePh and FP-OTf in MeCN- $d_{3}$ 
4.2 Synthesis of 2-phenyl-2-(3-phenyl-1-(phenylselanyl)propyl)tetrahydrofuran (10)

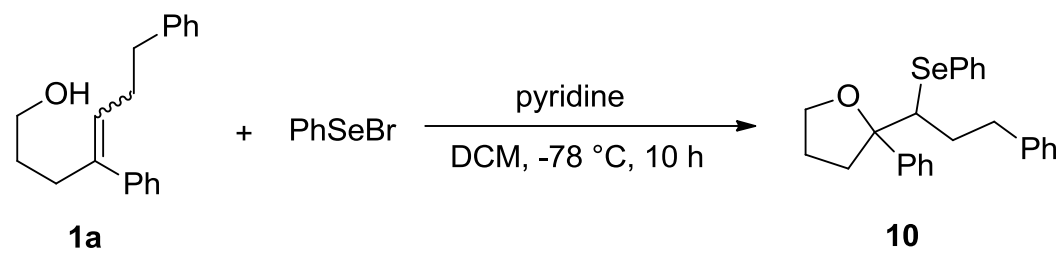

This is a modified literature procedure. ${ }^{11}$ An oven-dried $25 \mathrm{~mL}$ Schlenk flask equipped with a stir bar was evacuated and back-filled with nitrogen. Then, olefinic alcohol 1a (266 mg, $1 \mathrm{mmol})$ and anhydrous DCM (5 mL) was added and the solution was cooled to $-78{ }^{\circ} \mathrm{C}$. To the colorless solution were added pyridine $(88.79 \mu \mathrm{L}, 1.1$ mmol) and a solution of $\mathrm{PhSeBr}(260 \mathrm{mg}, 1.1 \mathrm{mmol})$ in anhydrous DCM (3 mL) via syringe. After stirred at $-78{ }^{\circ} \mathrm{C}$ for $10 \mathrm{~h}$, the resulting pale yellow solution was warmed to room temperature and concentrated in vacuo. The residue was purified by silica gel column chromatography (eluent: $\mathrm{PE}$ to PE/EtOAc $=30: 1, \mathrm{v} / \mathrm{v}$ ) to afford $\mathbf{1 0}$ as a colorless oil $(0.42 \mathrm{~g}, 99 \%$ yield, d.r. $=3: 1) .{ }^{1} \mathrm{H} \mathrm{NMR}\left(400 \mathrm{MHz}, \mathrm{CDCl}_{3}\right) \delta 7.57-$ $7.47(\mathrm{~m}, 4 \mathrm{H}), 7.32(\mathrm{t}, J=7.4 \mathrm{~Hz}, 3 \mathrm{H}), 7.26-7.18(\mathrm{~m}, 6 \mathrm{H}), 7.02(\mathrm{~d}, J=7.0 \mathrm{~Hz}, 2 \mathrm{H})$, $3.99-3.92(\mathrm{~m}, 1 \mathrm{H}), 3.83(\mathrm{td}, J=8.2,5.6 \mathrm{~Hz}, 1 \mathrm{H}), 3.37(\mathrm{dd}, J=10.6,2.9 \mathrm{~Hz}, 1 \mathrm{H})$, $3.03-2.93(\mathrm{~m}, 1 \mathrm{H}), 2.65-2.54(\mathrm{~m}, 1 \mathrm{H}), 2.51-2.42(\mathrm{~m}, 1 \mathrm{H}), 2.32-2.23(\mathrm{~m}, 2 \mathrm{H})$, $1.94-1.83(\mathrm{~m}, 1 \mathrm{H}), 1.79-1.69(\mathrm{~m}, 1 \mathrm{H}), 1.61-1.50(\mathrm{~m}, 1 \mathrm{H}) .{ }^{13} \mathrm{C}$ NMR $(101 \mathrm{MHz}$, $\left.\mathrm{CDCl}_{3}\right) \delta 143.7,142.0,134.0,129.1,128.7,128.3,128.2,127.9,127.2,127.1,126.8$, $125.8,89.6,67.7,58.2,37.3,34.7,34.4,25.7$. HR-ESI-MS m/z calcd for $\mathrm{C}_{25} \mathrm{H}_{26} \mathrm{OSe}$ $[\mathrm{M}+\mathrm{H}]^{+}:$423.1222, found: 423.1223 .

\subsection{Reaction of new selenium species and olefinic alcohol 1a}

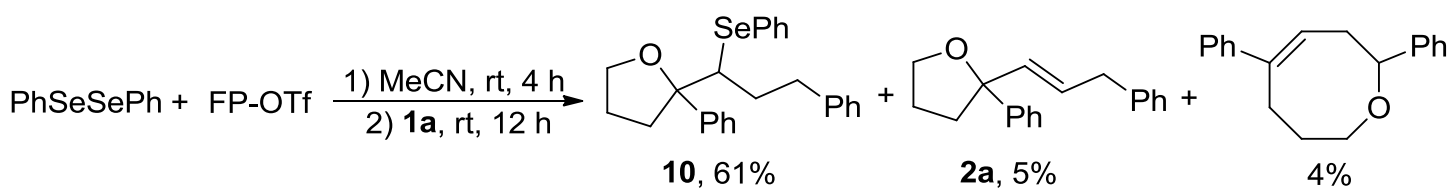

A 4-mL vial equipped with a magnetic stir bar was charged with FP-OTf (7.4 mg, $0.03 \mathrm{mmol})$ and $\mathrm{PhSeSePh}(9.4 \mathrm{mg}, 0.03 \mathrm{mmol})$. Then dried $\mathrm{MeCN}(1 \mathrm{~mL})$ was added, and the vial was capped. After stirred at room temperature for $4 \mathrm{~h}$, the resulting mixture was added to the solution of olfinic alcohol (1a) in dried MeCN (1 mL). The mixture was stirred at room temperature for additional $12 \mathrm{~h}$. The resulting mixture was concentrated in vacuo and analyzed by ${ }^{1} \mathrm{H}$ NMR spectroscopy in $\mathrm{CDCl}_{3}$. NMR yields were measured against benzyl benzoate as an internal standard. 


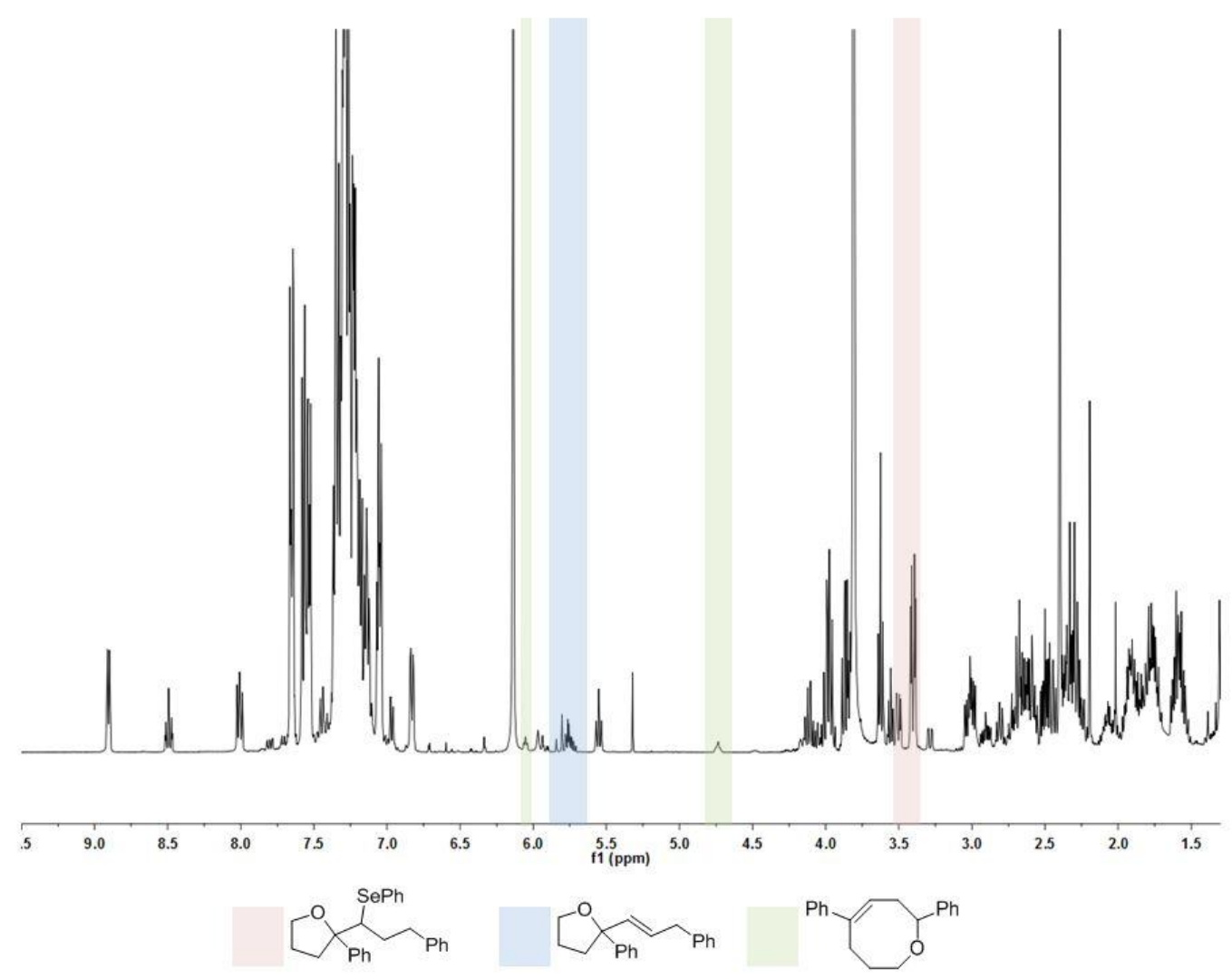

Scheme S4 Extract (9.50 to $1.35 \mathrm{ppm}$ ) of the ${ }^{1} \mathrm{H}$ NMR Spectrum of the Reaction Mixture

\subsection{FP-OTf vs PhSeX in oxidation of intermediate 10}

4.4.1 Oxidation of intermediate 10 through FP-OTf<smiles>[Se][Se]C(CCc1ccccc1)(c1ccccc1)C1CCCO1</smiles>

10

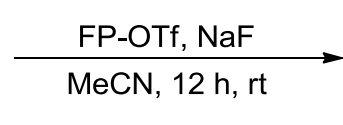

MeN, $12 \mathrm{~h}, \mathrm{rt}, \mathrm{g}$<smiles>C(=CC1(c2ccccc2)CCCO1)c1ccccc1</smiles>

2a

A 5-mL vial equipped with a magnetic stirrer bar was charged with FP-OTf ( $29.7 \mathrm{mg}$, $0.12 \mathrm{mmol}$ ), $\mathrm{NaF}$ (4.2 $\mathrm{mg}, 0.1 \mathrm{mmol})$ and $\mathbf{1 0}$ (42.1 $\mathrm{mg}, 0.1 \mathrm{mmol})$. Then dried MeCN $(2.5 \mathrm{~mL})$ was added, and the vial was capped. After the mixture was stirred at room temperature for $12 \mathrm{~h}$, a great deal of $\mathrm{PhSeSePh}$ was generated. The resulting yellow mixture was concentrated in vacuo. The residue was purified by silica gel column chromatography (eluent: $\mathrm{PE}$ to $\mathrm{PE} / \mathrm{EtOAc}=40: 1, \mathrm{v} / \mathrm{v}$ ) to afford the $\mathbf{2 a}$ as a pale yellow oil (21.6 mg, 82\%, contains $5 \%$ impurity which is difficult to separate).

\subsubsection{Oxidation of intermediate 10 through PhSeOTf}<smiles>c1ccc(CCC(c2ccccc2)C2(c3ccccc3)CCCO2)cc1</smiles>

10

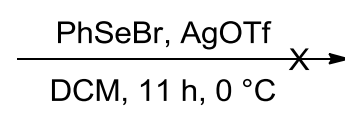

$\mathrm{DCM}, 11 \mathrm{~h}, 0^{\circ} \mathrm{C}$<smiles>C(=CC1(c2ccccc2)CCCO1)c1ccccc1</smiles>

$2 a$

PhSeOTf was prepared according to the literature. ${ }^{12}$ To an oven-dried $10 \mathrm{~mL}$ Schlenk flask equipped with a stir bar was added $\mathrm{PhSeBr}(18.2 \mathrm{mg}, 0.077 \mathrm{mmol})$. The flask 
was then evacuated and back-filled with nitrogen. Next, anhydrous DCM (1 mL) was added via syringe and cooled to $0{ }^{\circ} \mathrm{C}$. To the brown solution was added AgOTf (19.8 $\mathrm{mg}, 0.077 \mathrm{mmol}$ ) in one portion and the heterogeneous mixture was stirred at $0{ }^{\circ} \mathrm{C}$ for $10 \mathrm{~min}$. Then, the mixture was added to a solution of $\mathbf{1 0}(29.5 \mathrm{mg}, 0.07 \mathrm{mmol})$ in anhydrous DCM $(1 \mathrm{~mL})$. After stirred at $0{ }^{\circ} \mathrm{C}$ for $11 \mathrm{~h}$, the resulting mixture was concentrated in vacuo and analyzed by ${ }^{1} \mathrm{H}$ NMR spectroscopy in $\mathrm{CDCl}_{3}$.

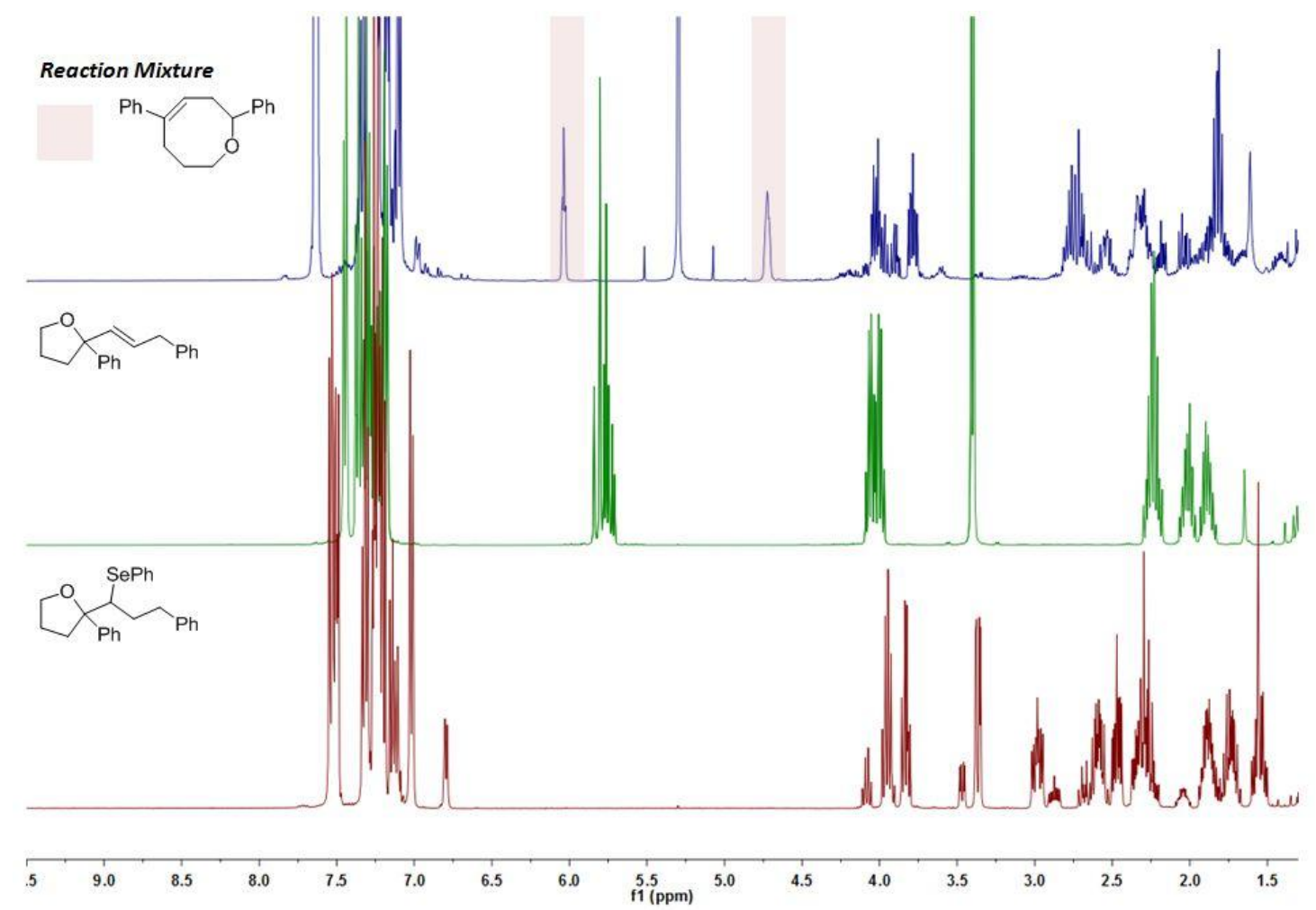

Scheme S5 Extract (9.50 to $1.30 \mathrm{ppm}$ ) of the ${ }^{1} \mathrm{H}$ NMR Spectra of the Reaction Mixture 
<smiles>c1ccc(CCC(c2ccccc2)C2(c3ccccc3)CCCO2)cc1</smiles>

10
$\frac{\mathrm{PhSeBr}, \mathrm{AgOTf}, \mathrm{NaF}}{\mathrm{DCM}, 11 \mathrm{~h}, 0{ }^{\circ} \mathrm{C}} \leftrightarrow \longrightarrow$

2a

To an oven-dried $10 \mathrm{~mL}$ Schlenk flask equipped with a stir bar was added $\mathrm{PhSeBr}$ (18.2 $\mathrm{mg}, 0.077 \mathrm{mmol}$ ). The flask was then evacuated and back-filled with nitrogen. Next, anhydrous DCM (1 mL) was added via syringe and cooled to $0{ }^{\circ} \mathrm{C}$. To the brown solution was added AgOTf $(19.8 \mathrm{mg}, 0.077 \mathrm{mmol})$ in one portion and the heterogeneous mixture was stirred at $0{ }^{\circ} \mathrm{C}$ for $10 \mathrm{~min}$. Then, the mixture was added to a solution of $10(29.5 \mathrm{mg}, 0.07 \mathrm{mmol})$ and $\mathrm{NaF}(3.2 \mathrm{mg}, 0.077 \mathrm{mmol})$ in anhydrous DCM $(1 \mathrm{~mL})$. After stirred at $0{ }^{\circ} \mathrm{C}$ for $11 \mathrm{~h}$, the resulting mixture was concentrated in vacuo and analyzed by ${ }^{1} \mathrm{H}$ NMR spectroscopy in $\mathrm{CDCl}_{3}$.

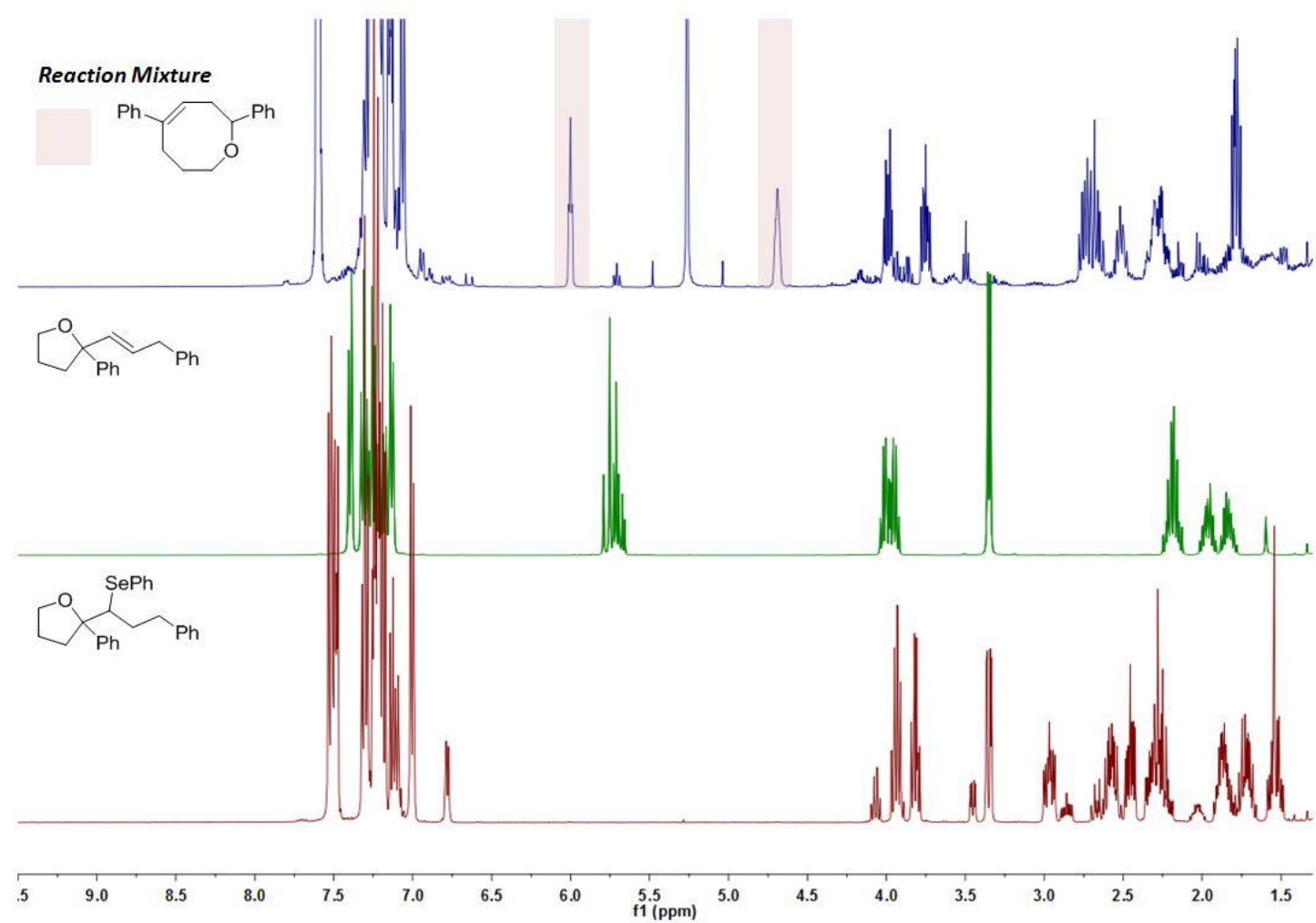

Scheme S6 Extract (9.50 to $1.30 \mathrm{ppm}$ ) of the ${ }^{1} \mathrm{H}$ NMR Spectra of the Reaction Mixture 
<smiles>c1ccc(CCC(c2ccccc2)C2(c3ccccc3)CCCO2)cc1</smiles>

10

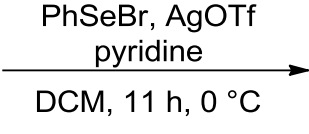

DCM, $11 \mathrm{~h}, 0^{\circ} \mathrm{C}$

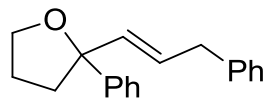

2a, trace

PhSeOTf was prepared according to the literature. ${ }^{13}$ To an oven-dried $10 \mathrm{~mL}$ Schlenk flask equipped with a stir bar was added $\mathrm{PhSeBr}(18.2 \mathrm{mg}, 0.077 \mathrm{mmol})$. The flask was then evacuated and back-filled with nitrogen. Next, anhydrous DCM (1 mL) was added via syringe and cooled to $0{ }^{\circ} \mathrm{C}$. To the brown solution was added AgOTf (19.8 $\mathrm{mg}, 0.077 \mathrm{mmol}$ ) in one portion and the heterogeneous mixture was stirred at $0{ }^{\circ} \mathrm{C}$ for $10 \mathrm{~min}$. Then, the mixture was added to a solution of $10(29.5 \mathrm{mg}, 0.07 \mathrm{mmol})$ and pyridine $(6.2 \mu \mathrm{L}, 0.077 \mathrm{mmol})$ in anhydrous DCM $(1 \mathrm{~mL})$. After stirred at $0{ }^{\circ} \mathrm{C}$ for 11 $\mathrm{h}$, the resulting mixture was concentrated in vacuo and analyzed by ${ }^{1} \mathrm{H}$ NMR spectroscopy in $\mathrm{CDCl}_{3}$.

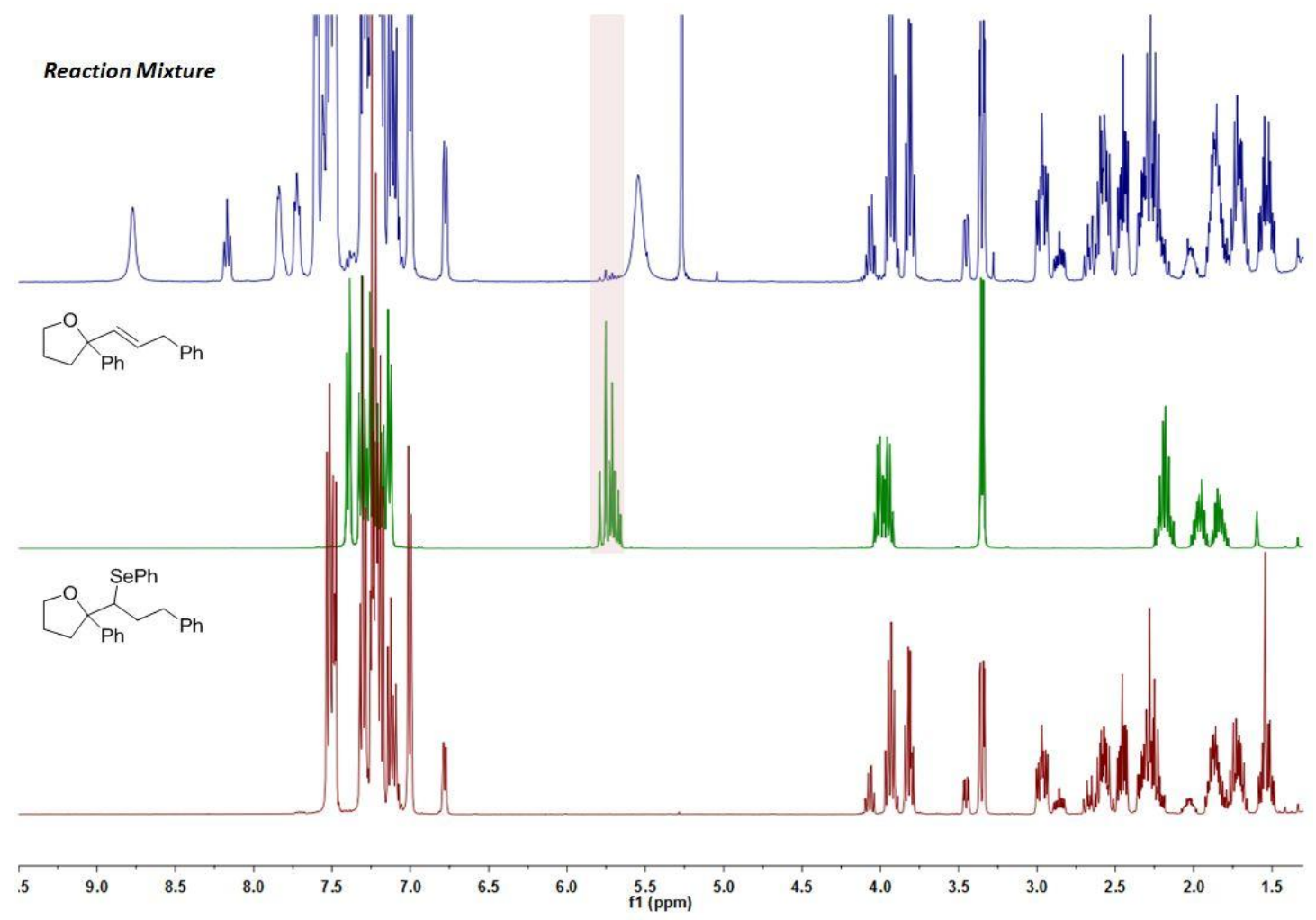

Scheme S7 Extract (9.50 to $1.30 \mathrm{ppm})$ of the ${ }^{1} \mathrm{H}$ NMR Spectra of the Reaction Mixture 


\subsubsection{Oxidation of intermediate 10 through $\mathrm{PhSeBr}$}<smiles>c1ccc(CCC(c2ccccc2)C2(c3ccccc3)CCCO2)cc1</smiles>

10

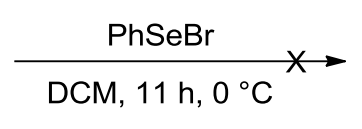

$\mathrm{DCM}, 11 \mathrm{~h}, 0^{\circ} \mathrm{C}$<smiles>C(=CC1(c2ccccc2)CCCO1)Cc1ccccc1</smiles>

$2 a$

An oven-dried $10 \mathrm{~mL}$ Schlenk flask equipped with a stir bar was evacuated and back-filled with nitrogen. Then, $10(29.5 \mathrm{mg}, 0.07 \mathrm{mmol})$ and anhydrous DCM (1 mL) was added and the colorless solution was cooled to $0{ }^{\circ} \mathrm{C}$. Then a solution of $\mathrm{PhSeBr}$ $(18.2 \mathrm{mg}, 0.077 \mathrm{mmol})$ in DCM $(1 \mathrm{~mL})$ was added. After stirred at $0{ }^{\circ} \mathrm{C}$ for $11 \mathrm{~h}$, the resulting mixture was concentrated in vacuo and analyzed by ${ }^{1} \mathrm{H}$ NMR spectroscopy in $\mathrm{CDCl}_{3}$.

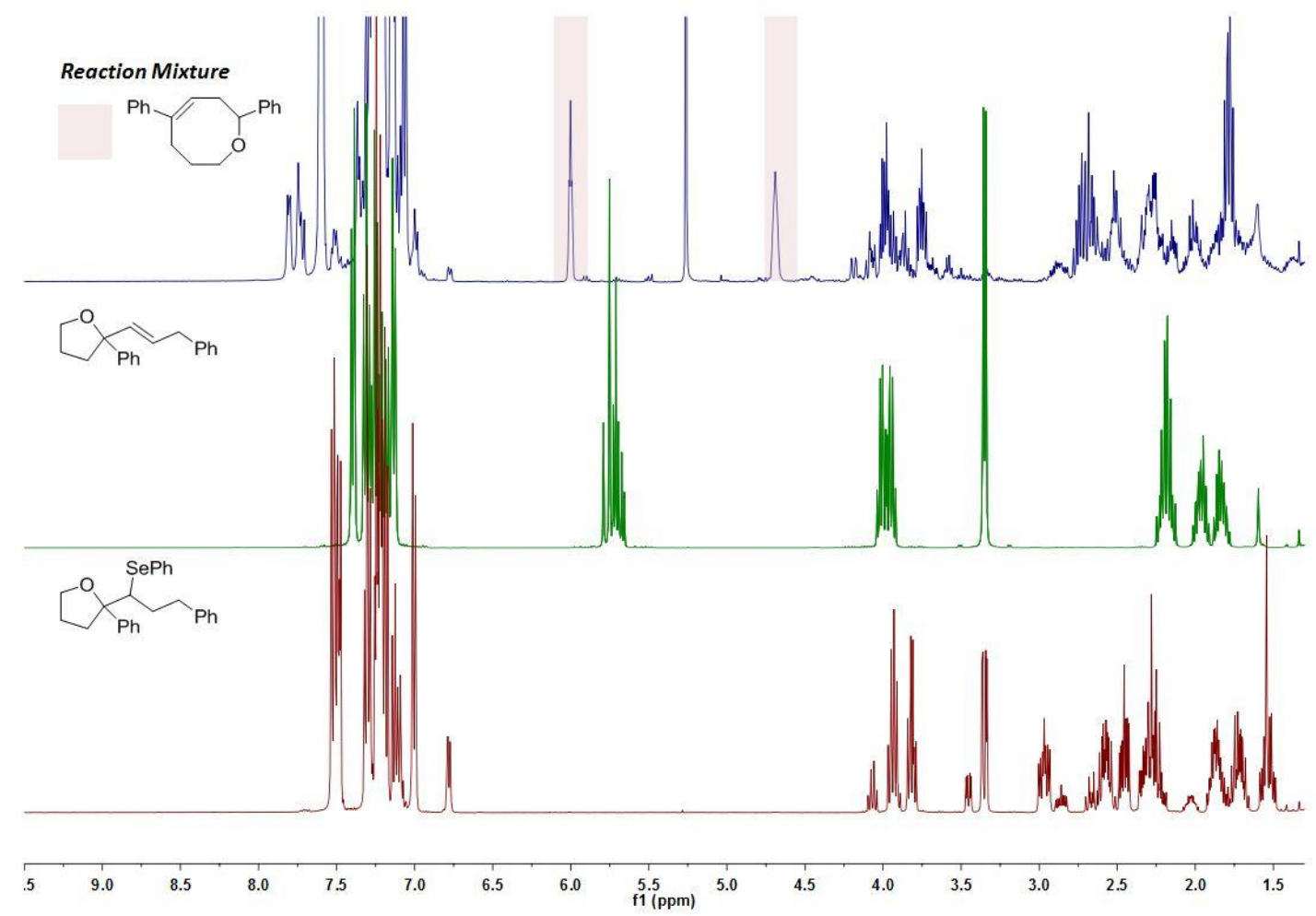

Scheme S8 Extract (9.50 to $1.30 \mathrm{ppm}$ ) of the ${ }^{1} \mathrm{H}$ NMR Spectra of the Reaction Mixture 


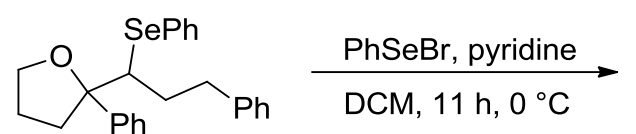

10

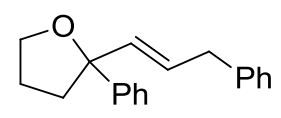

2a, trace

An oven-dried $10 \mathrm{~mL}$ Schlenk flask equipped with a stir bar was evacuated and back-filled with nitrogen. Then, $\mathbf{1 0}(29.5 \mathrm{mg}, 0.07 \mathrm{mmol})$, pyridine $(6.2 \mu \mathrm{L}, 0.077$ mmol) and anhydrous DCM $(1 \mathrm{~mL})$ was added and the colorless solution was cooled to $0{ }^{\circ} \mathrm{C}$. Then a solution of $\mathrm{PhSeBr}(18.2 \mathrm{mg}, 0.077 \mathrm{mmol})$ in $\mathrm{DCM}(1 \mathrm{~mL})$ was added. After stirred at $0{ }^{\circ} \mathrm{C}$ for $11 \mathrm{~h}$, the resulting mixture was concentrated in vacuo and analyzed by ${ }^{1} \mathrm{H}$ NMR spectroscopy in $\mathrm{CDCl}_{3}$.

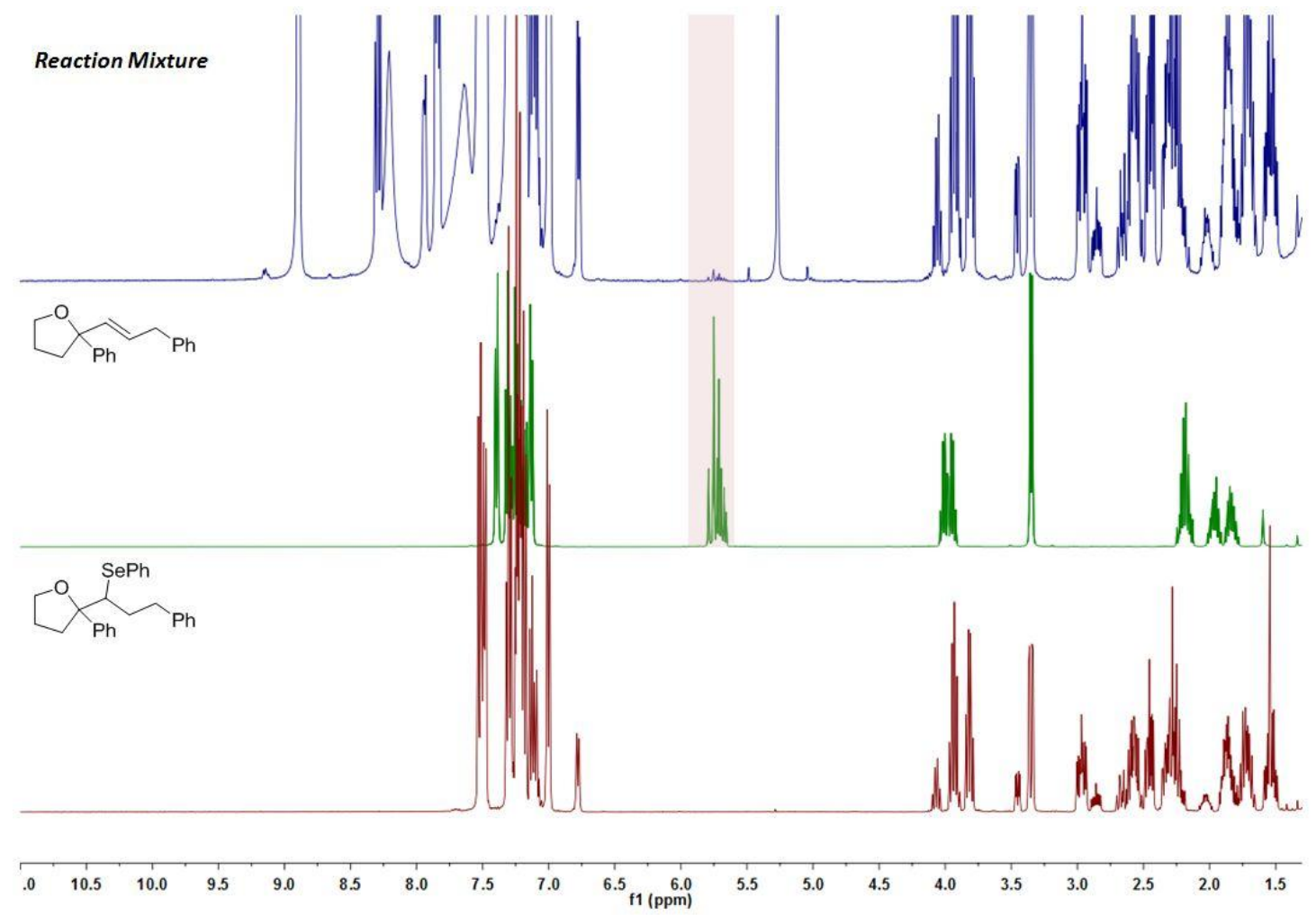

Scheme S9 Extract (11.0 to $1.30 \mathrm{ppm}$ ) of the ${ }^{1} \mathrm{H}$ NMR Spectra of the Reaction Mixture 


\subsubsection{Oxidation of intermediate 10 through PhSeF}<smiles>c1ccc(CCC(c2ccccc2)C2(c3ccccc3)CCCO2)cc1</smiles>

10

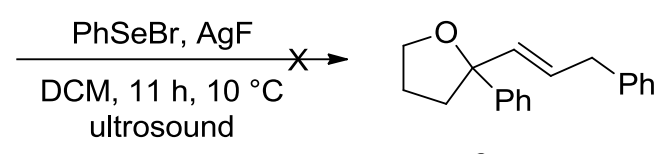

2a

PhSeF was prepared according to the literature. ${ }^{14}$ To an oven-dried $10 \mathrm{~mL}$ Schlenk flask equipped with a stir bar was added $\mathrm{PhSeBr}(18.2 \mathrm{mg}, 0.077 \mathrm{mmol})$. The flask was then evacuated and back-filled with nitrogen. Next, anhydrous DCM (1 mL) was added via syringe and cooled to $10^{\circ} \mathrm{C}$. To the brown solution was added $\mathrm{AgF}$ (9.8 $\mathrm{mg}$, $0.077 \mathrm{mmol}$ ) in one portion and the heterogeneous mixture was irradiated with ultrasound at $10{ }^{\circ} \mathrm{C}$ for $30 \mathrm{~min}$. Then, the mixture was added to a solution of $\mathbf{1 0}$ (29.5 $\mathrm{mg}, 0.07 \mathrm{mmol})$ in anhydrous DCM $(1 \mathrm{~mL})$. After stirred at $10^{\circ} \mathrm{C}$ for $11 \mathrm{~h}$, the resulting mixture was concentrated in vacuo and analyzed by ${ }^{1} \mathrm{H}$ NMR spectroscopy in $\mathrm{CDCl}_{3}$.

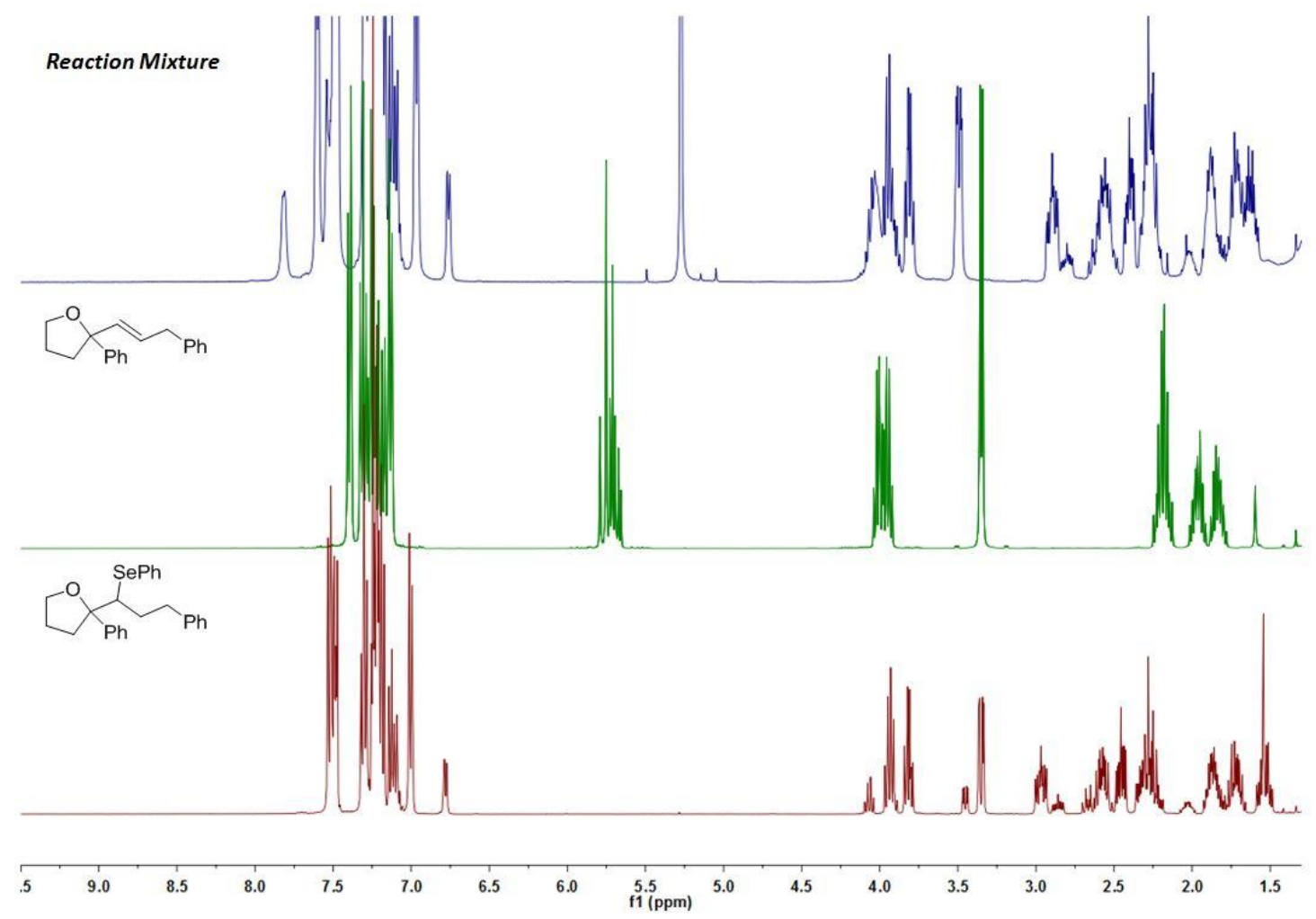

Scheme S10 Extract (9.50 to $1.30 \mathrm{ppm})$ of the ${ }^{1} \mathrm{H}$ NMR Spectra of the Reaction Mixture 


\section{Reference}

(1) Ota, K.; Kurokawa, T.; Kawashima, E.; Miyaoka, H. Tetrahedron 2009, 65, 8668.

(2) Abouabdellah, A.; Bonnet-Delpon, D. Tetrahedron 1994, 41, 11921.

(3) Snider, B. B.; Lobera, M.; Marien, T. P. J. Org. Chem. 2003, 68, 6451.

(4) Tan, C. K.; Zhou, L.; Yeung, Y.-Y. Org. Lett. 2011, 13, 2738.

(5) Gruenanger, C. U.; Breit, B. Angew. Chem. Int. Ed. 2010, 49, 967.

(6) Chen, F.; Tan, C. K.; Yeung, Y.-Y. J. Am. Soc. Chem. 2013, 135, 1232.

(7) Kurth, M. J.; Decker, O. H. W. J. Org. Chem. 1985, 50, 5769.

(8) DeBoef, B.; Counts, W. R.; Gilbertson, S. R. J. Org. Chem. 2007, 72, 799.

(9) Zawisza, A.; Fenet, B.; Sinou, D. Eur. J. Org. Chem. 2007, 2296.

(10) Denmark, S. E.; Edwards, M. G. J. Org. Chem. 2006, 71, 7293.

(11) Murata, S.; Suzuki, T. Chem. Lett. 1987, 849.

(12) Tomoda, S.; Usuki, Y. Chem. Lett. 1989, 1235. 
6. NMR spectra for new compounds

${ }^{1} \mathrm{H}$ NMR (400 MHz, $\left.\mathrm{CDCl}_{3}\right)$ and $\left.{ }^{13} \mathrm{C} \mathrm{NMR} \mathrm{(101} \mathrm{MHz,} \mathrm{CDCl}_{3}\right)$ of $1 \mathrm{~b}$

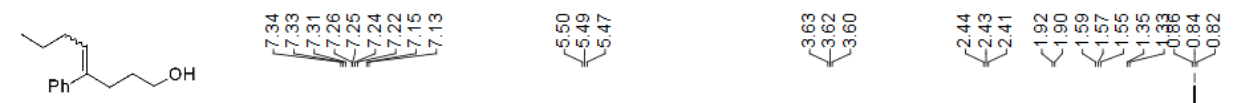

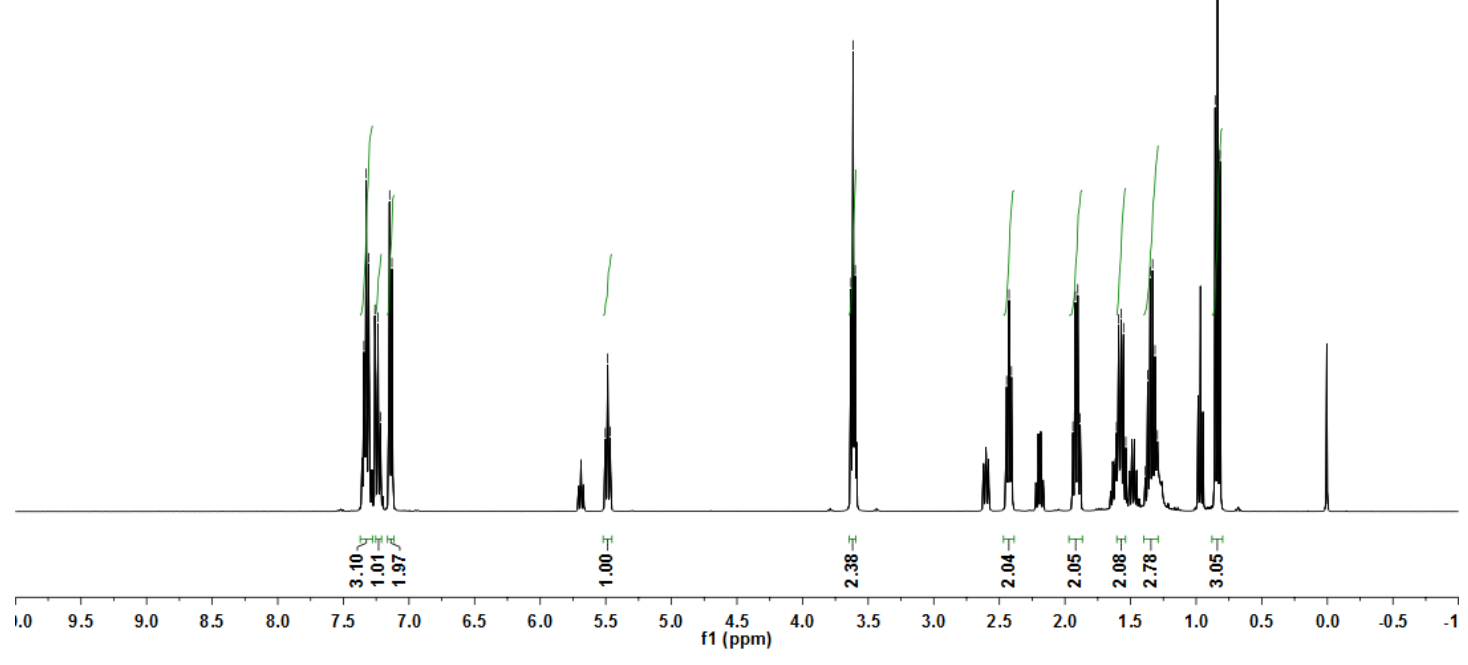

حOH

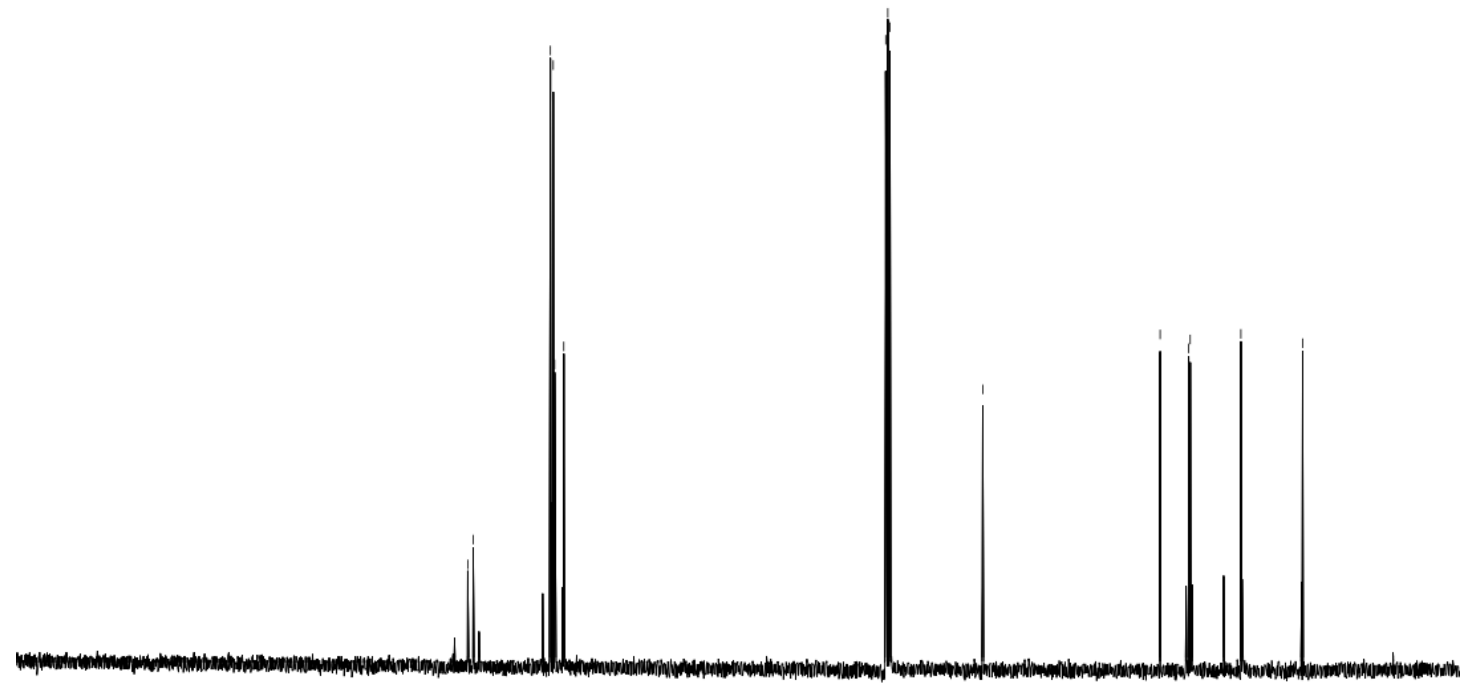

\begin{tabular}{llllllllllllllllllllllllll}
\hline 10 & 200 & 190 & 180 & 170 & 160 & 150 & 140 & 130 & 120 & 110 & 100 & 90 & 80 & 70 & 60 & 50 & 40 & 30 & 20 & 10 & 0 & -1
\end{tabular} 
${ }^{1} \mathrm{H}$ NMR (400 MHz, $\left.\mathrm{CDCl}_{3}\right)$ and ${ }^{13} \mathrm{C}$ NMR (101 MHz, $\left.\mathrm{CDCl}_{3}\right)$ of 1c
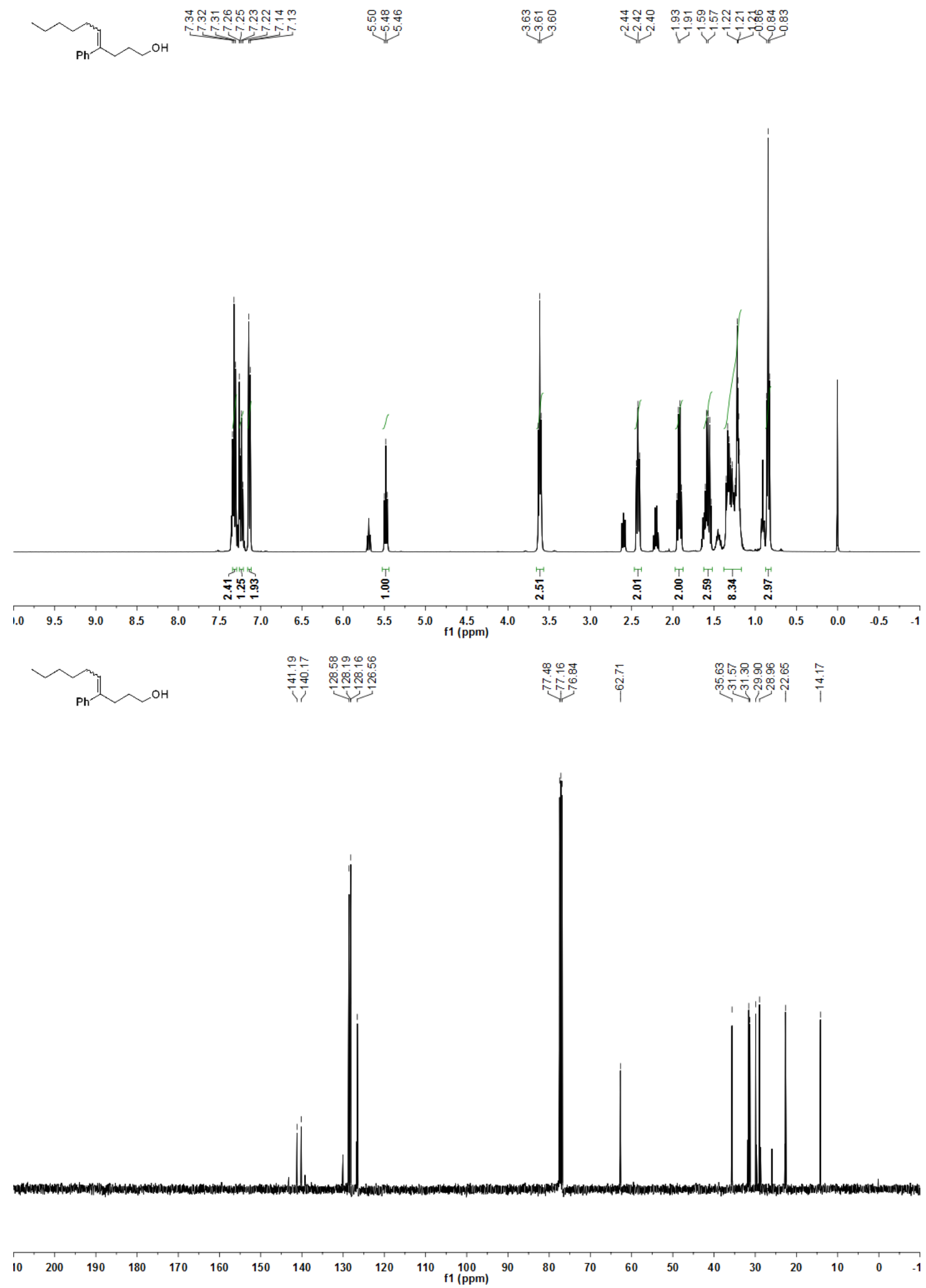
${ }^{1} \mathrm{H}$ NMR (400 MHz, $\left.\mathrm{CDCl}_{3}\right)$ and ${ }^{13} \mathrm{C}$ NMR (101 MHz, $\left.\mathrm{CDCl}_{3}\right)$ of $1 \mathrm{e}$
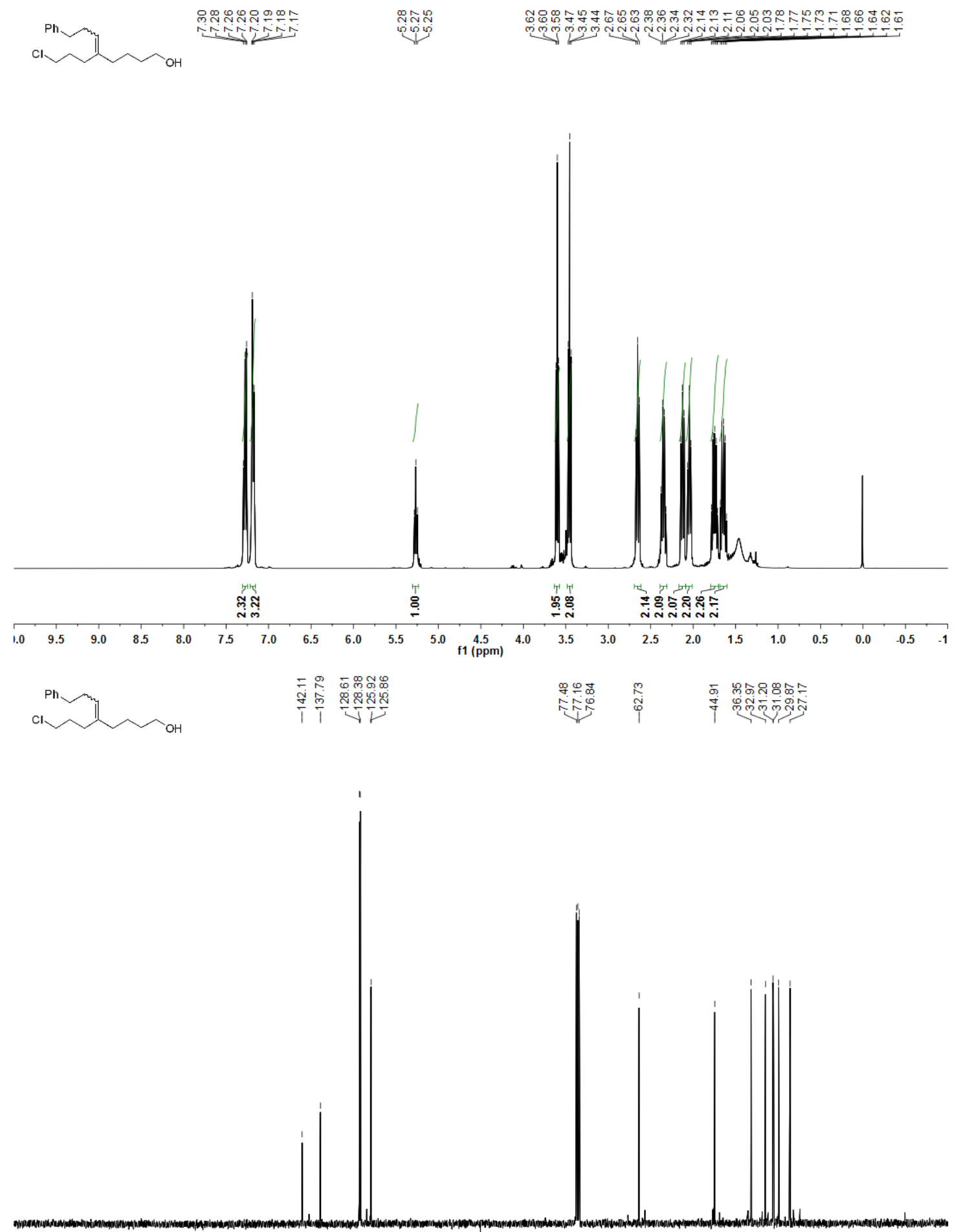

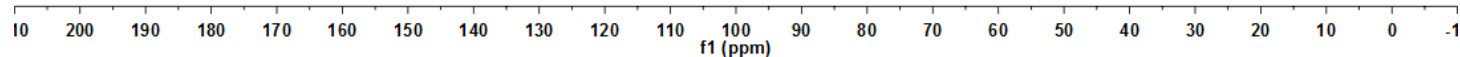


${ }^{1} \mathrm{H}$ NMR (400 MHz, $\mathrm{CDCl}_{3}$ ) and ${ }^{13} \mathrm{C}$ NMR (101 MHz, $\left.\mathrm{CDCl}_{3}\right)$ of $1 f$
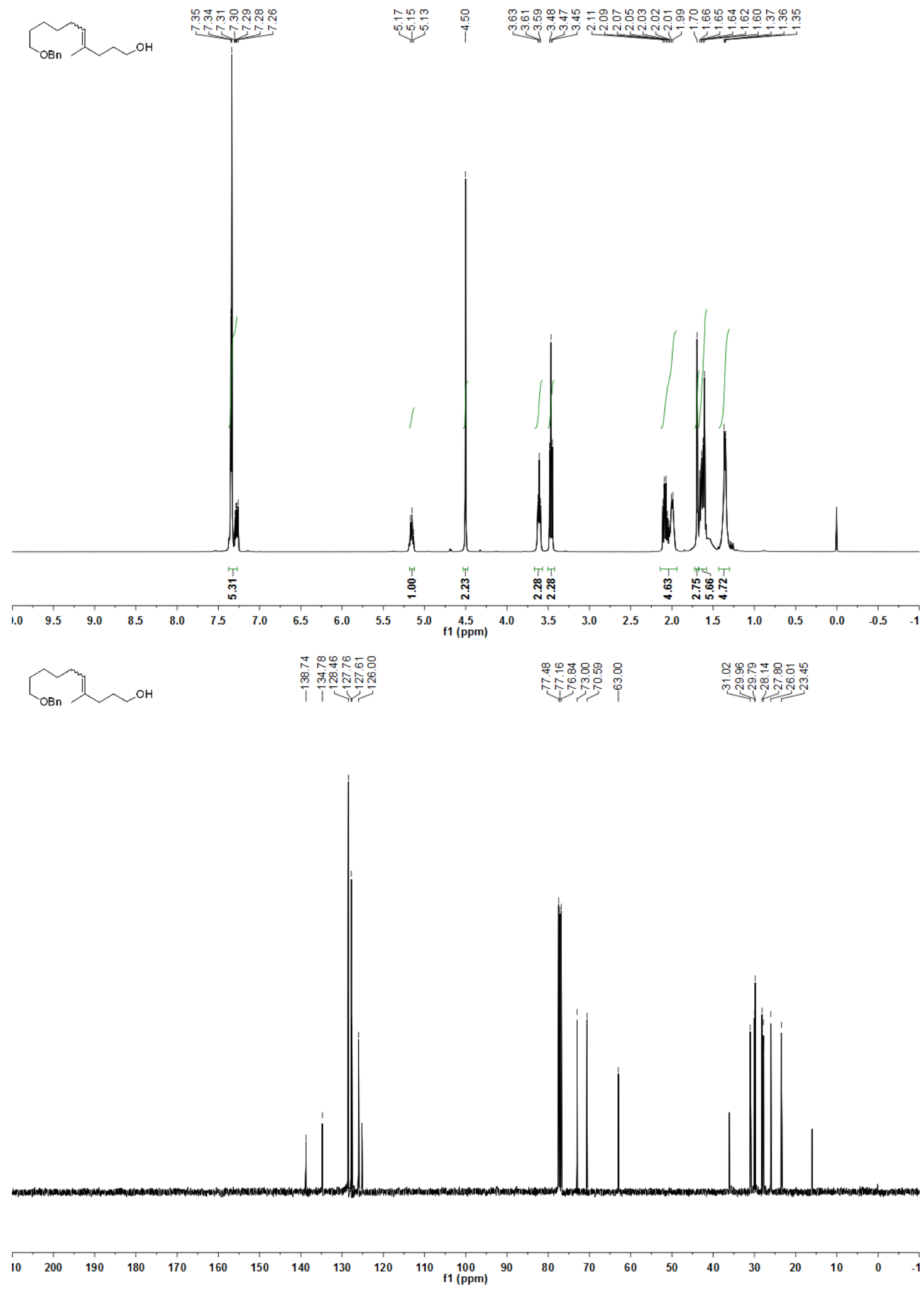
${ }^{1} \mathrm{H}$ NMR (400 MHz, $\left.\mathrm{CDCl}_{3}\right)$ and ${ }^{13} \mathrm{C}$ NMR (101 MHz, $\left.\mathrm{CDCl}_{3}\right)$ of $1 \mathrm{~h}$
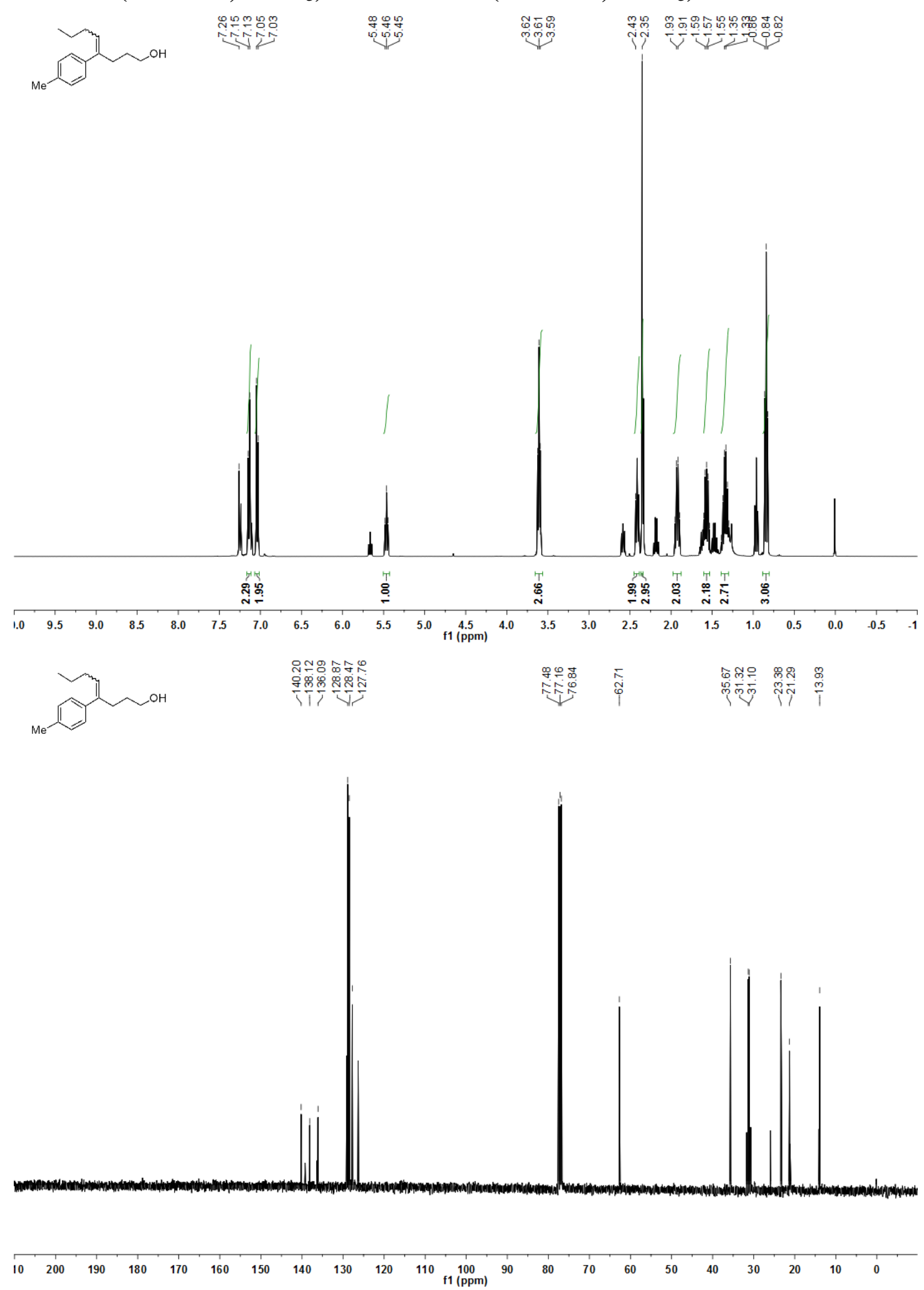
${ }^{1} \mathrm{H}$ NMR (400 MHz, $\left.\mathrm{CDCl}_{3}\right)$ and ${ }^{13} \mathrm{C}$ NMR (101 MHz, $\left.\mathrm{CDCl}_{3}\right)$ of $1 \mathrm{i}$
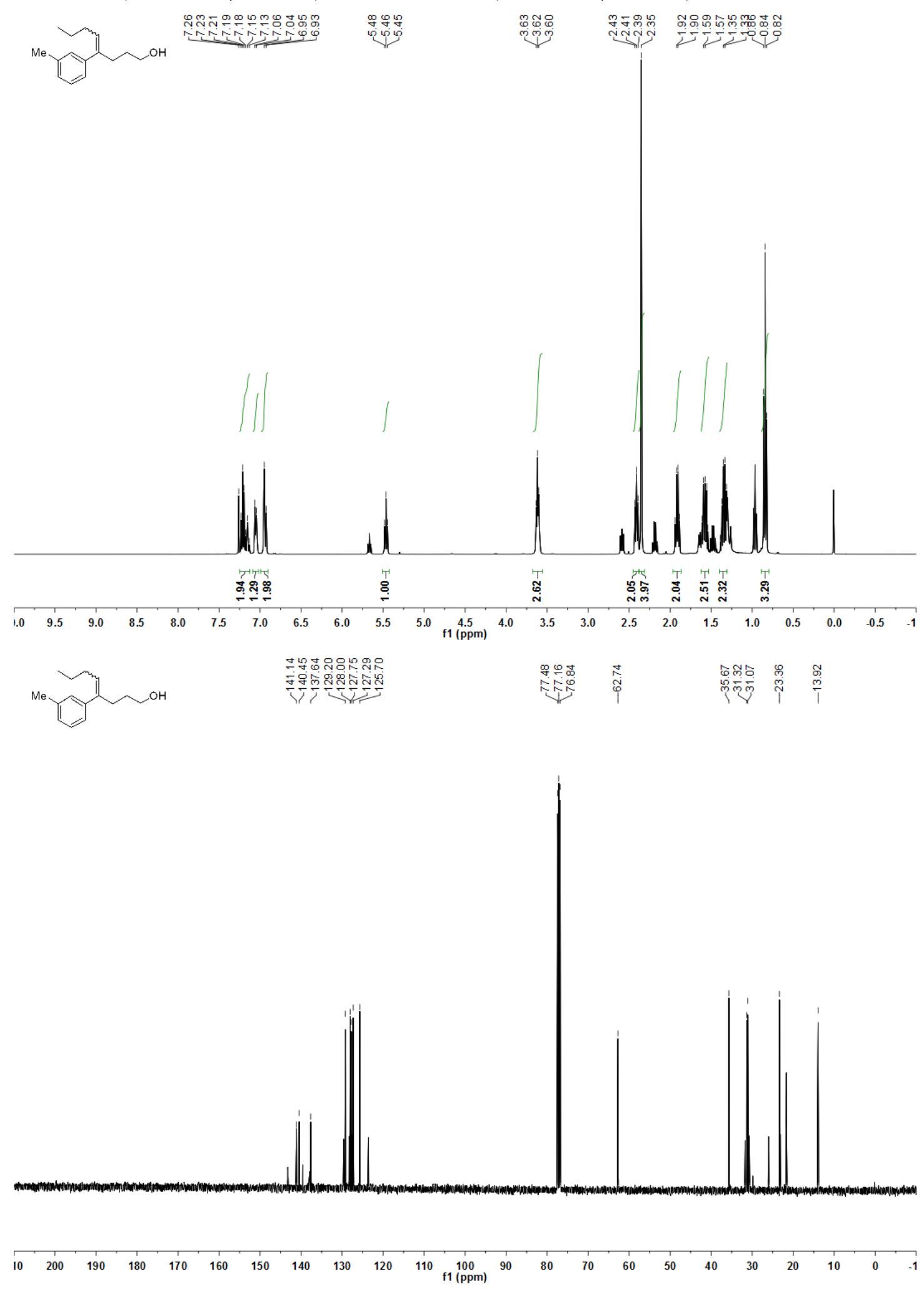
${ }^{1} \mathrm{H}$ NMR $\left(400 \mathrm{MHz}, \mathrm{CDCl}_{3}\right)$ and $\left.{ }^{13} \mathrm{C} \mathrm{NMR} \mathrm{(101} \mathrm{MHz,} \mathrm{CDCl}_{3}\right)$ of $1 \mathrm{j}$
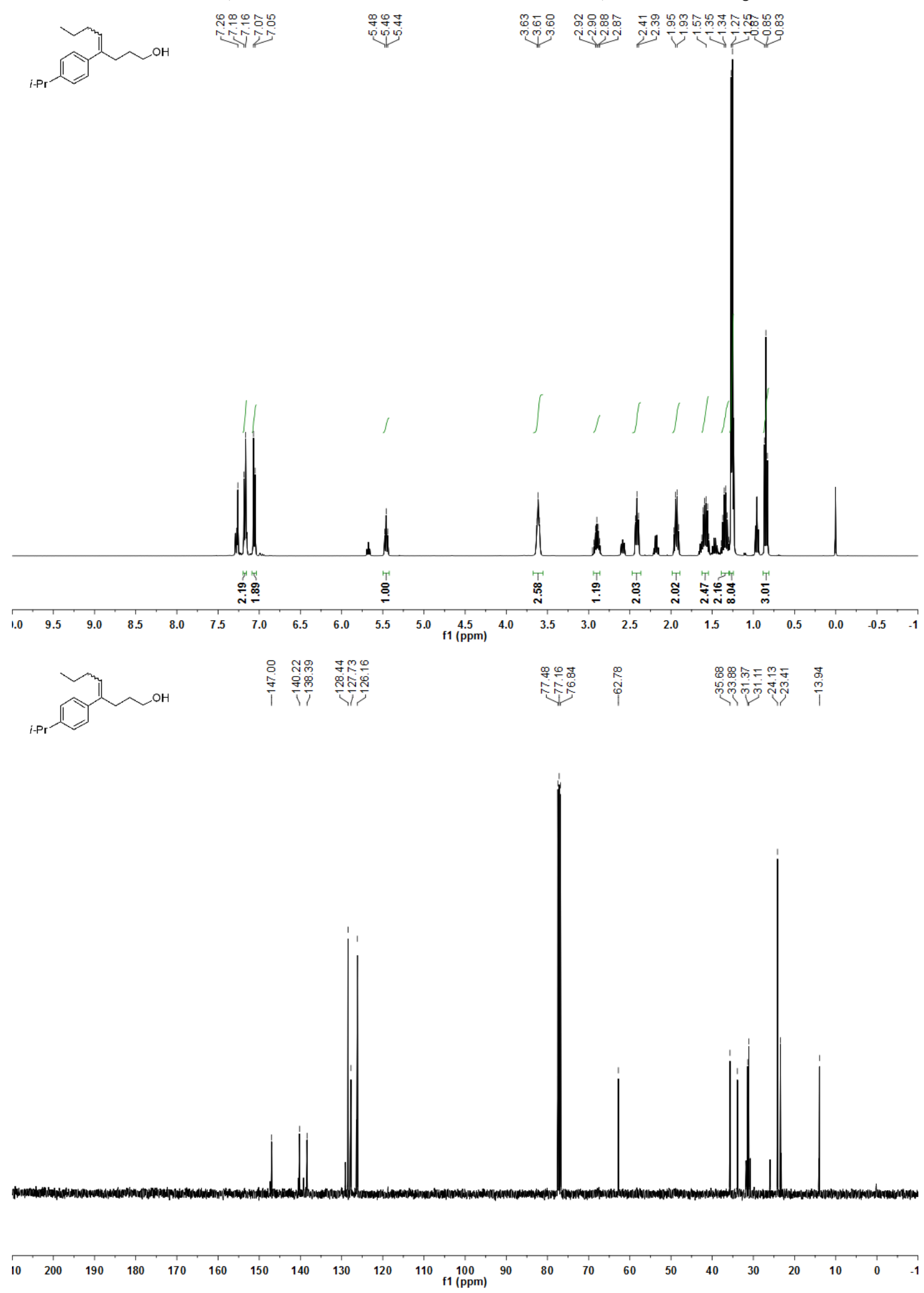
${ }^{1} \mathrm{H}$ NMR (400 MHz, $\left.\mathrm{CDCl}_{3}\right)$ and ${ }^{13} \mathrm{C}$ NMR (101 MHz, $\left.\mathrm{CDCl}_{3}\right)$ of $1 \mathrm{k}$
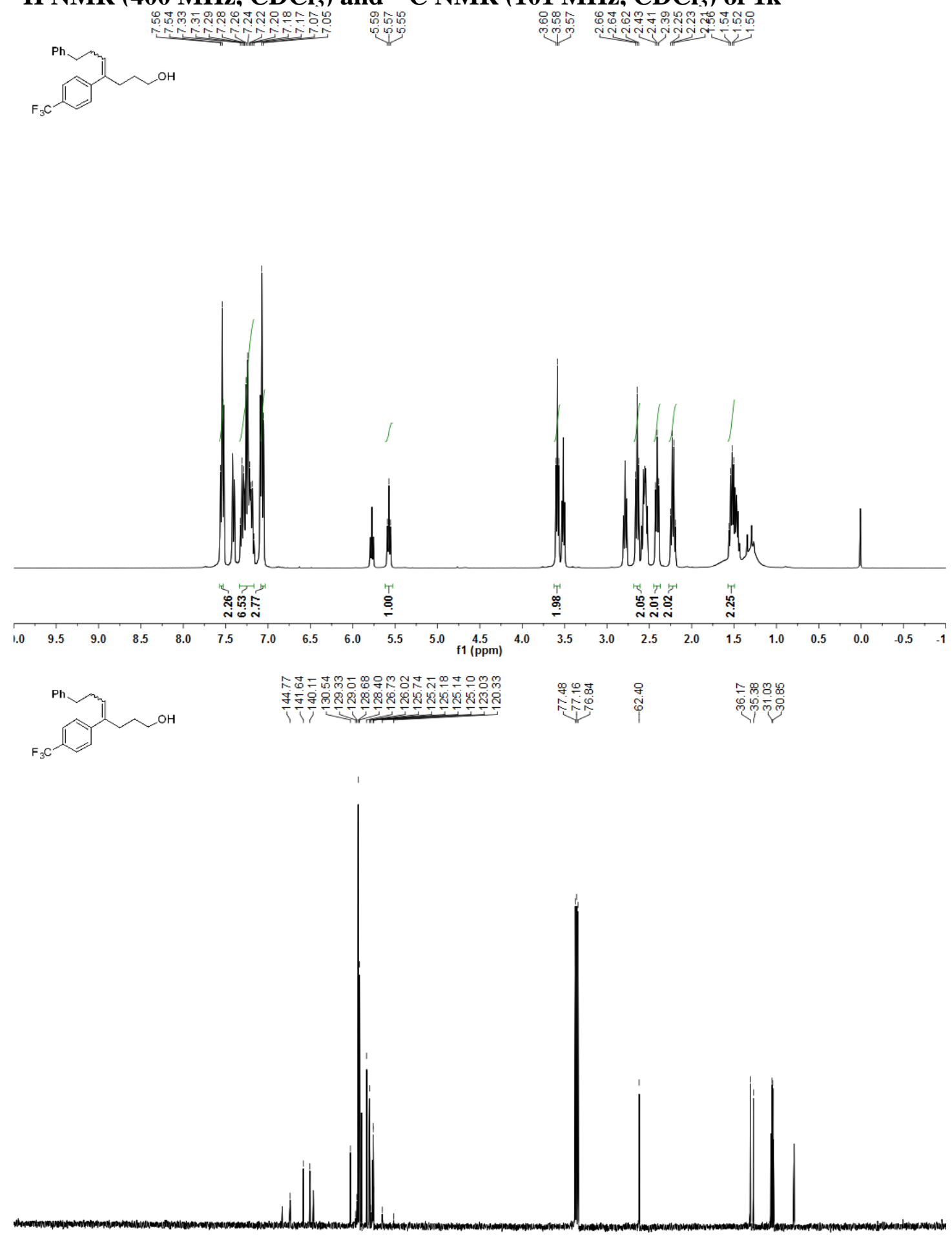

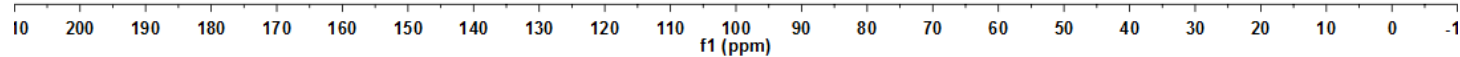


${ }^{1} \mathrm{H}$ NMR (400 MHz, $\left.\mathrm{CDCl}_{3}\right)$ and ${ }^{13} \mathrm{C}$ NMR (101 MHz, $\left.\mathrm{CDCl}_{3}\right)$ of $1 \mathrm{l}$
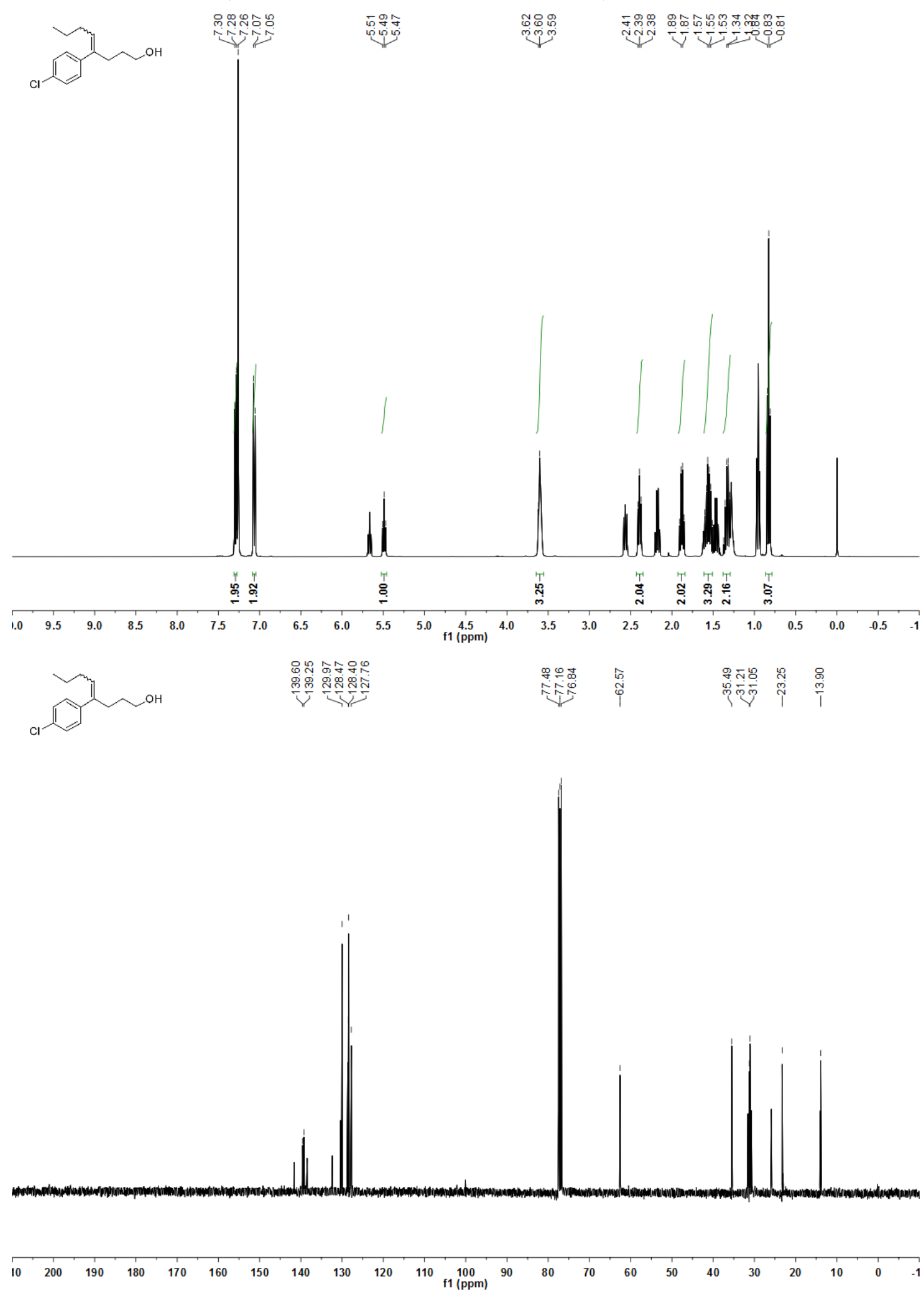
${ }^{1} \mathrm{H}$ NMR $\left(400 \mathrm{MHz}, \mathrm{CDCl}_{3}\right)$ and ${ }^{13} \mathrm{C}$ NMR $\left(101 \mathrm{MHz}, \mathrm{CDCl}_{3}\right)$ of $1 \mathrm{~m}$
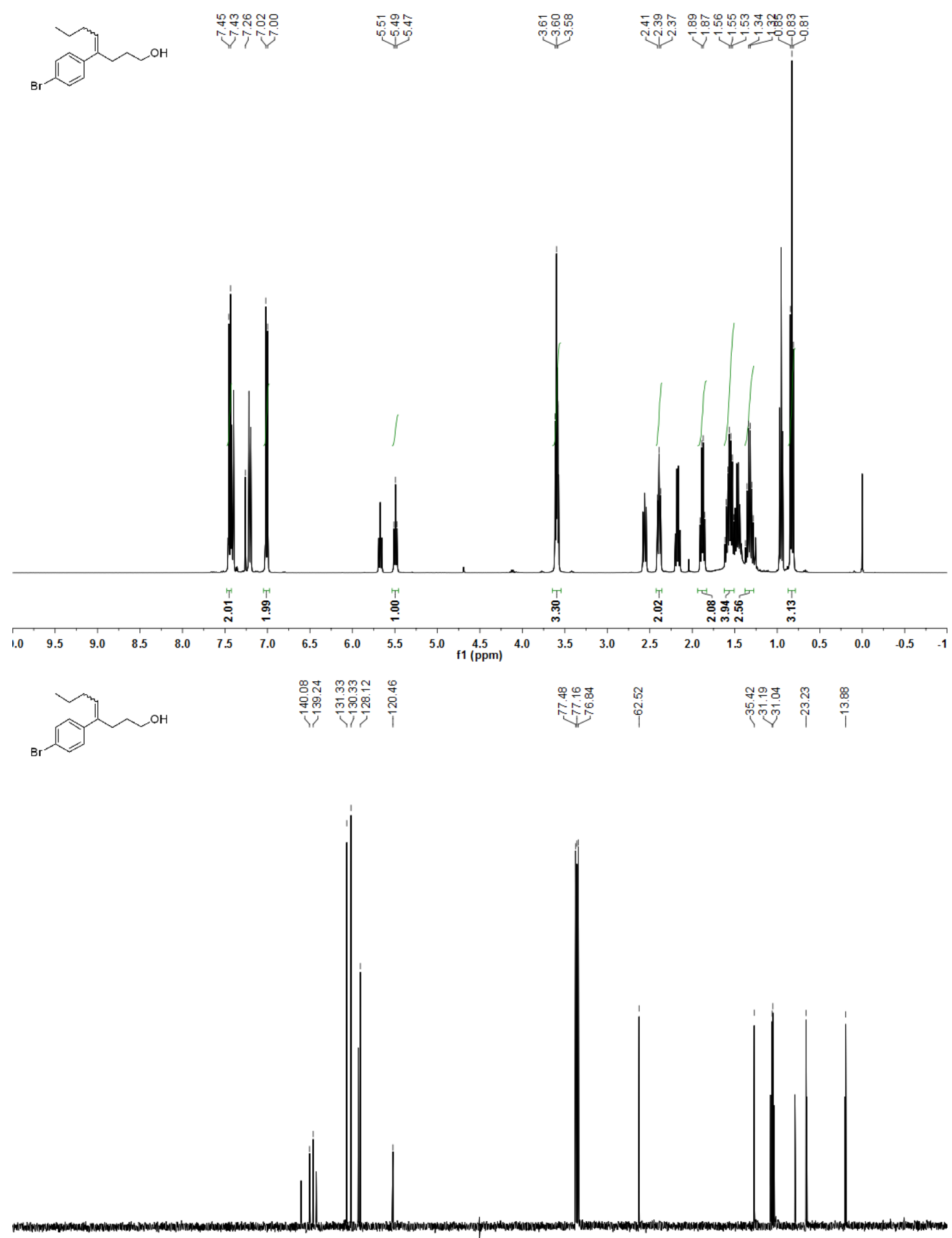

\begin{tabular}{|l|lllllllllllllllllllllllll}
\hline 10 & 200 & 190 & 180 & 170 & 160 & 150 & 140 & 130 & 120 & 110 & $\begin{array}{c}100 \\
\mathrm{f} 1(\mathrm{ppm})\end{array}$ & 90 & 80 & 70 & 60 & 50 & 40 & 30 & 20 & 10 & 0 & -1
\end{tabular} 
${ }^{1} \mathrm{H}$ NMR (400 MHz, $\left.\mathrm{CDCl}_{3}\right)$ and ${ }^{13} \mathrm{C}$ NMR $\left(101 \mathrm{MHz}, \mathrm{CDCl}_{3}\right)$ of $1 \mathrm{n}$
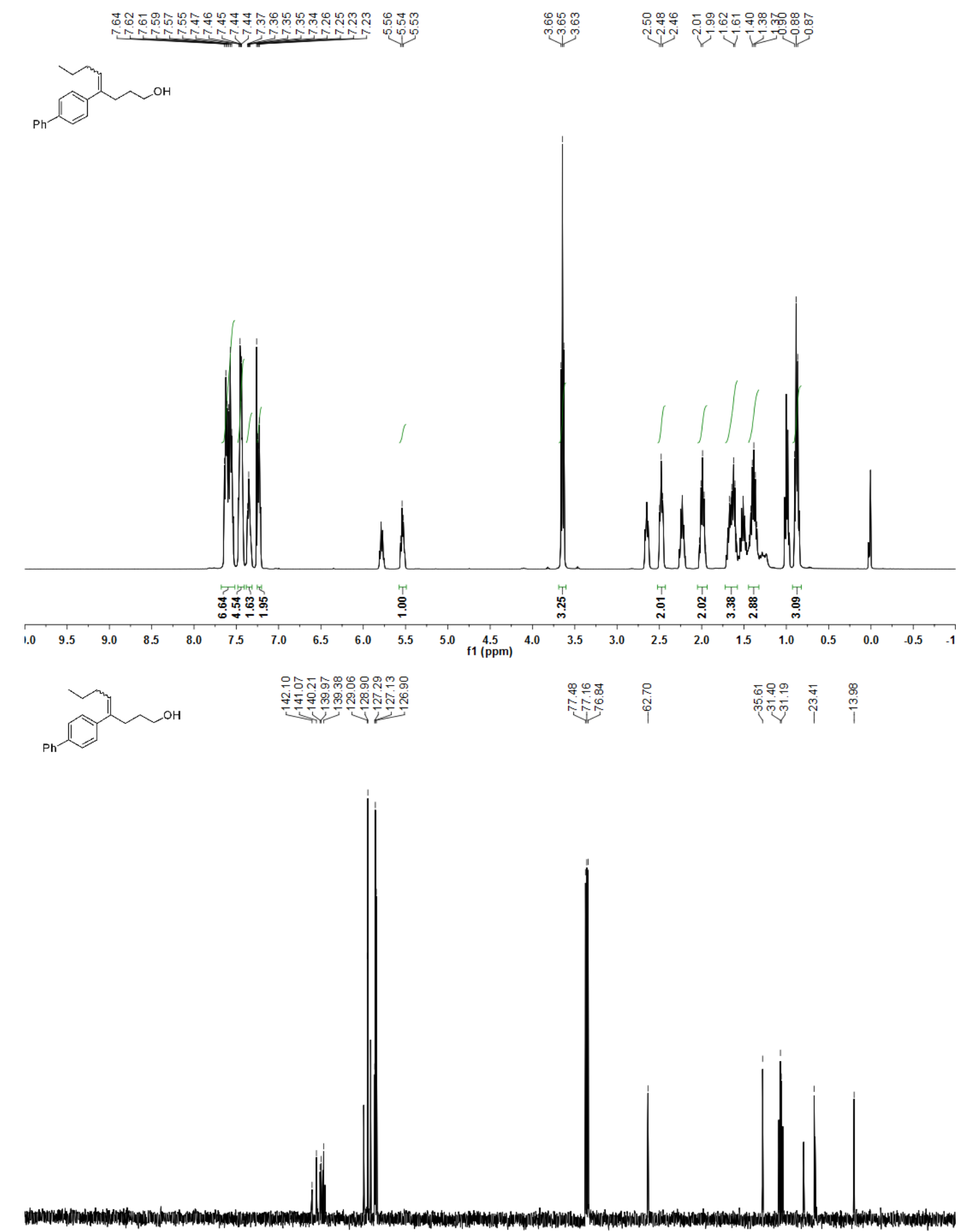

\begin{tabular}{|l|llllllllllllllllllllllll}
\hline 10 & 200 & 190 & 180 & 170 & 160 & 150 & 140 & 130 & 120 & 110 & $\begin{array}{c}100 \\
\mathrm{f} 1(\mathrm{ppm})\end{array}$ & 90 & 80 & 70 & 60 & 50 & 40 & 30 & 20 & 10 & 0 & -1
\end{tabular} 
${ }^{1} \mathrm{H}$ NMR (400 MHz, $\left.\mathrm{CDCl}_{3}\right)$ and ${ }^{13} \mathrm{C}$ NMR (101 MHz, $\left.\mathrm{CDCl}_{3}\right)$ of 10
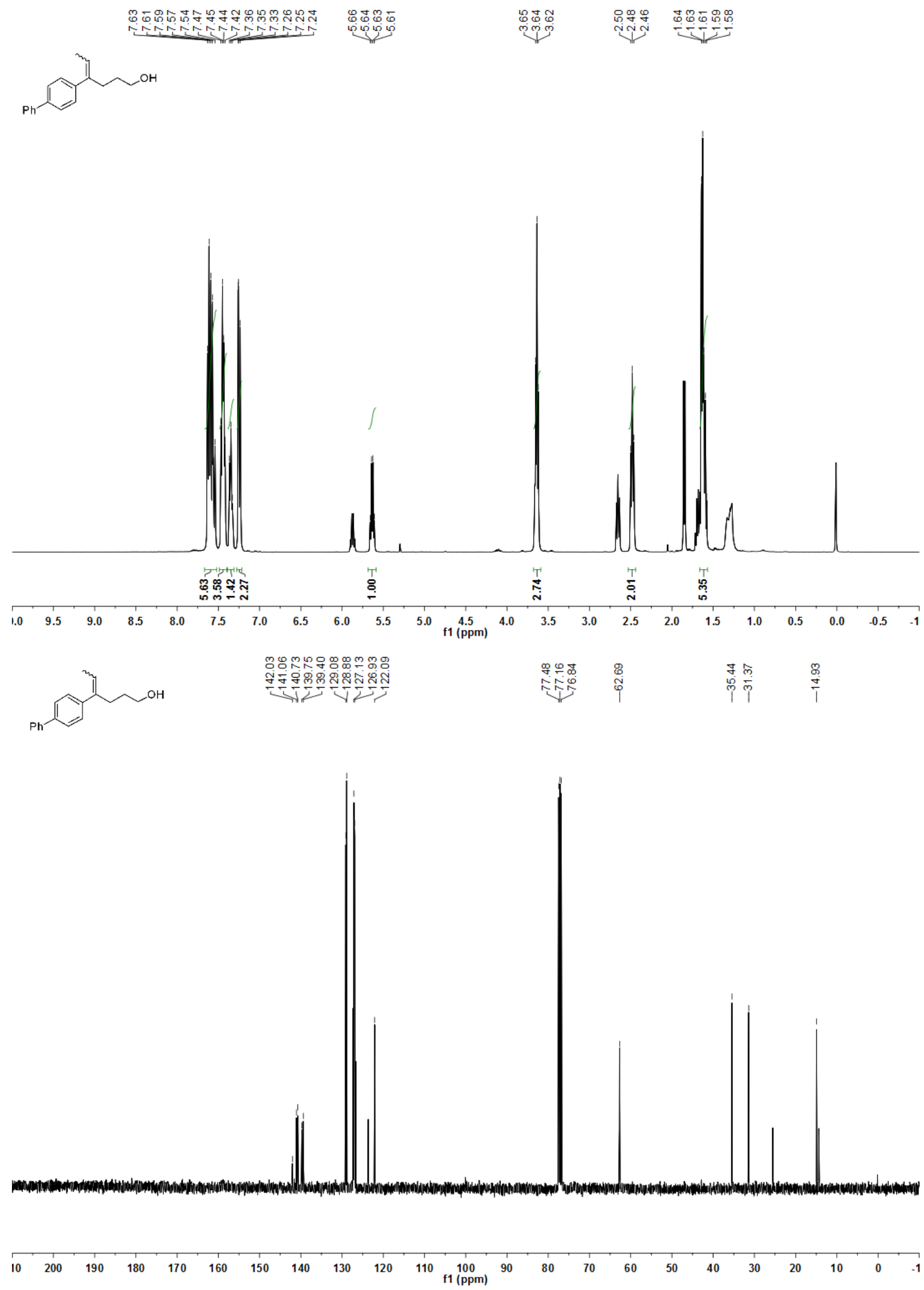
${ }^{1} \mathrm{H}$ NMR (400 MHz, $\left.\mathrm{CDCl}_{3}\right)$ and ${ }^{13} \mathrm{C}$ NMR $\left(101 \mathrm{MHz}, \mathrm{CDCl}_{3}\right)$ of $1 \mathrm{p}$
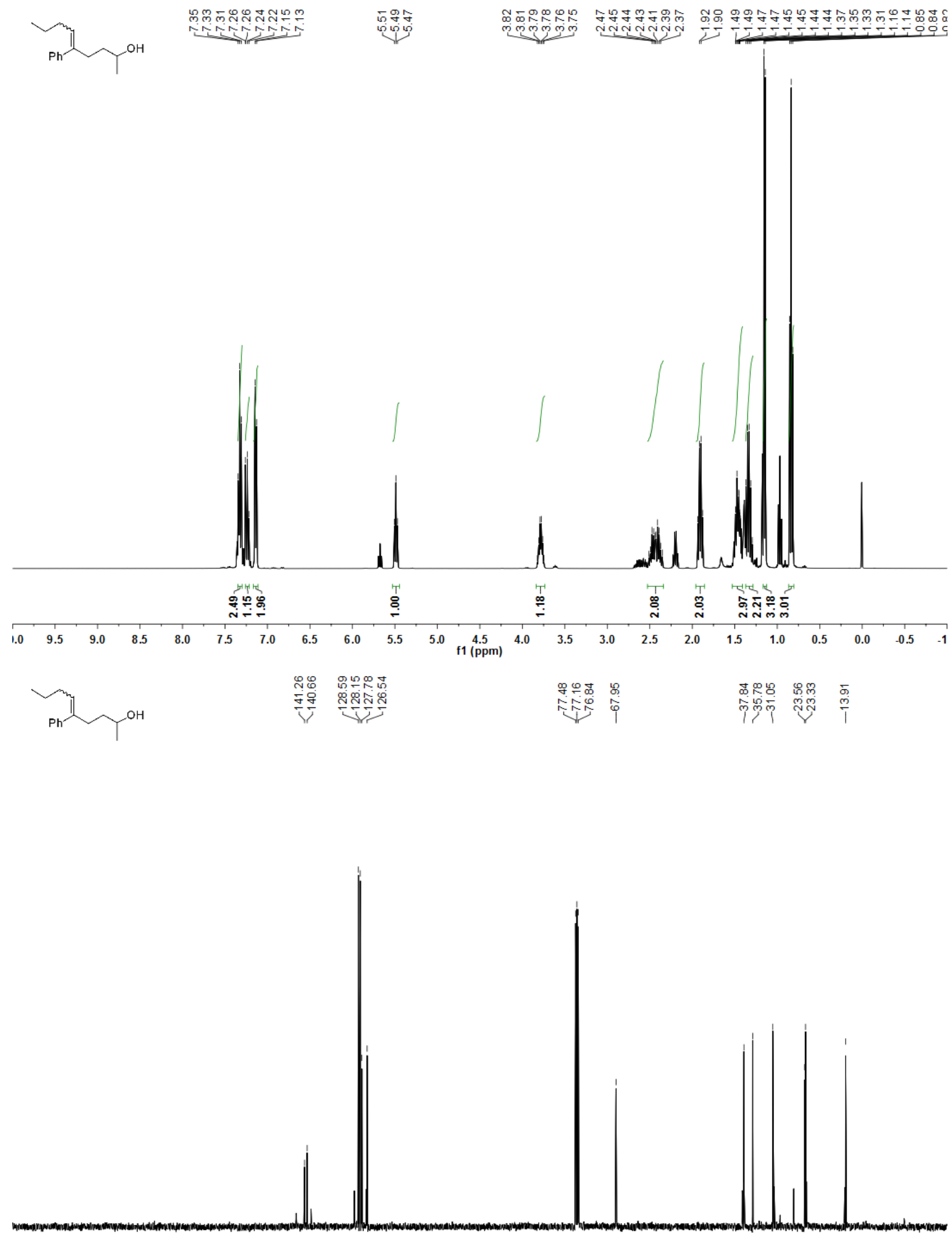

\begin{tabular}{|l|llllllllllllllllllllllll}
\hline 10 & 200 & 190 & 180 & 170 & 160 & 150 & 140 & 130 & 120 & 110 & $\begin{array}{c}100 \\
\mathrm{f} 1(\mathrm{ppm})\end{array}$ & 90 & 80 & 70 & 60 & 50 & 40 & 30 & 20 & 10 & 0 & -1
\end{tabular} 
${ }^{1} \mathrm{H}$ NMR (400 MHz, $\left.\mathrm{CDCl}_{3}\right)$ and ${ }^{13} \mathrm{C}$ NMR (101 $\left.\mathrm{MHz}, \mathrm{CDCl}_{3}\right)$ of 1q
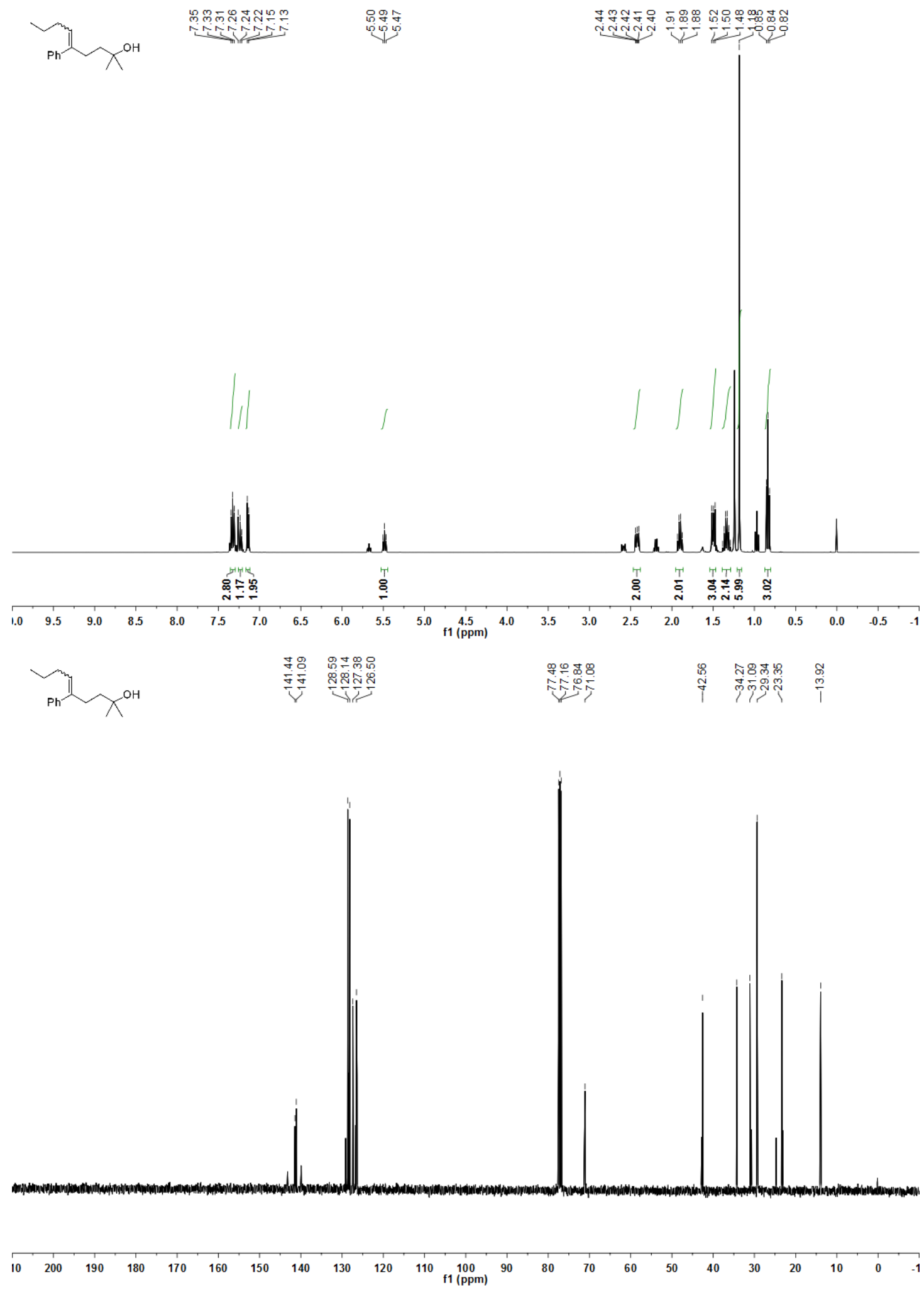
${ }^{1} \mathrm{H}$ NMR (400 MHz, $\mathrm{CDCl}_{3}$ ) and ${ }^{13} \mathrm{C}$ NMR (101 MHz, $\left.\mathrm{CDCl}_{3}\right)$ of $1 \mathrm{r}$
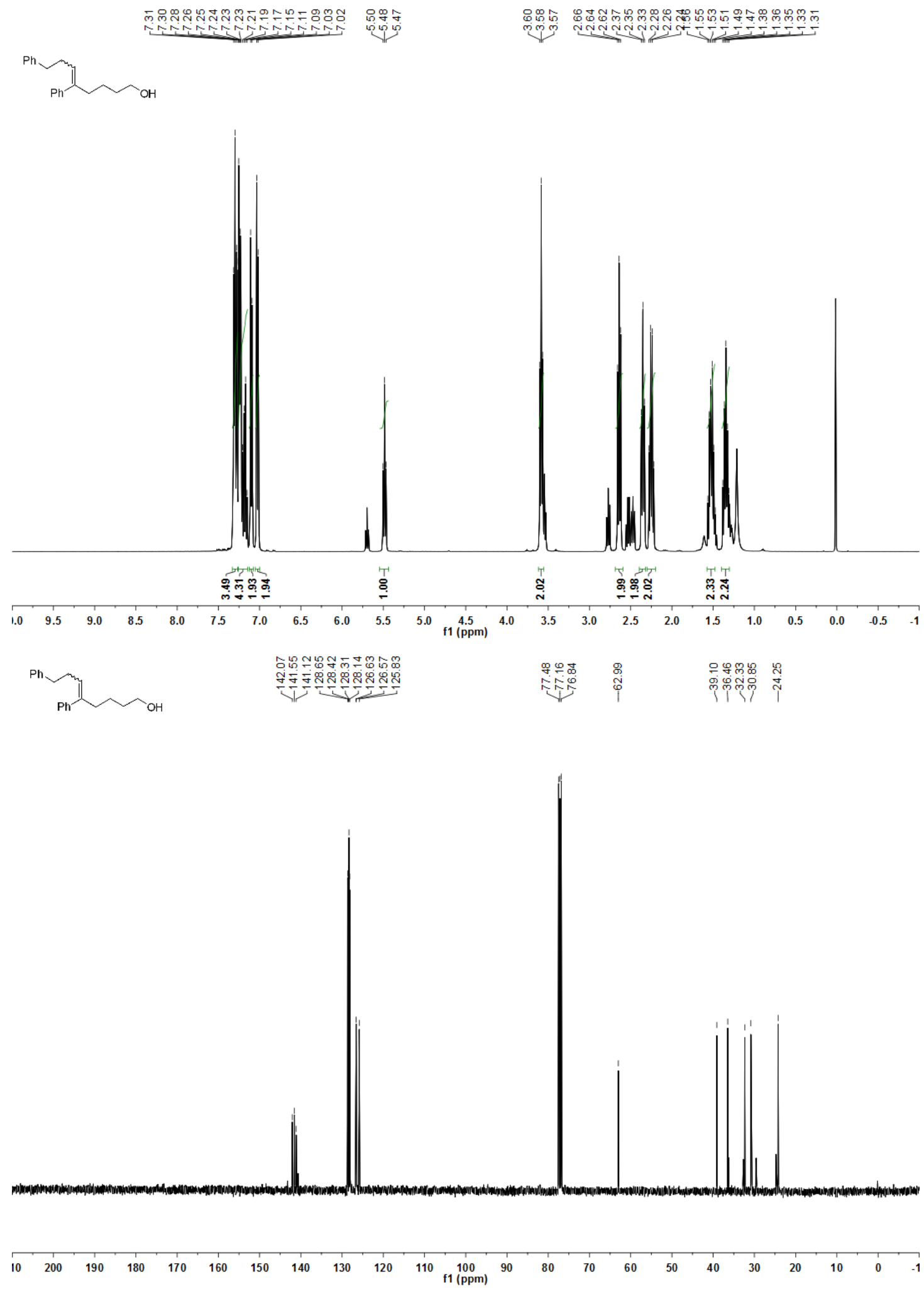
${ }^{1} \mathrm{H}$ NMR (400 MHz, $\left.\mathrm{CDCl}_{3}\right)$ and ${ }^{13} \mathrm{C}$ NMR (101 MHz, $\left.\mathrm{CDCl}_{3}\right)$ of $1 \mathrm{~s}$

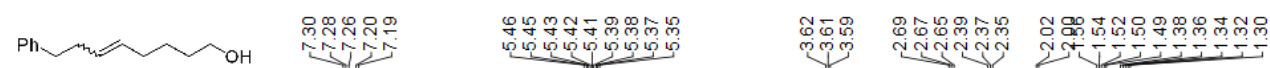

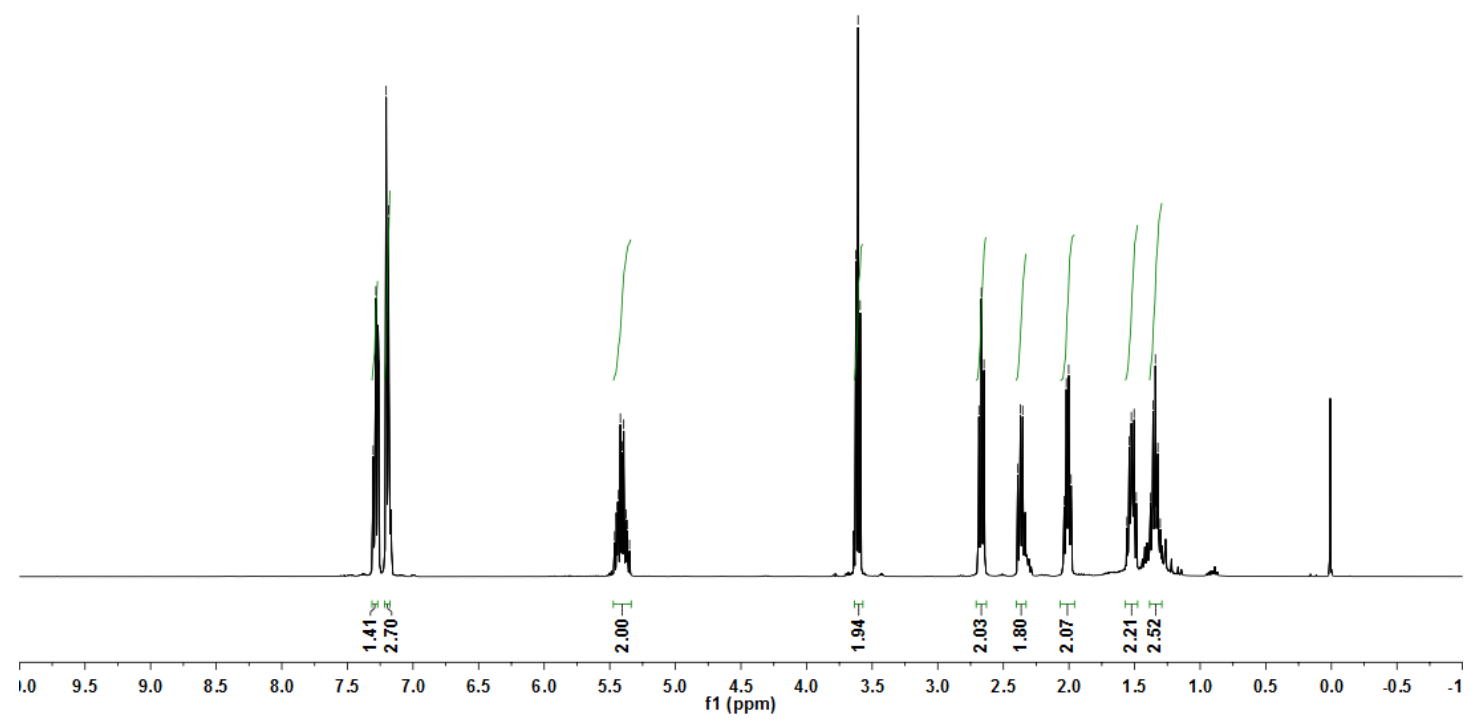

مOH

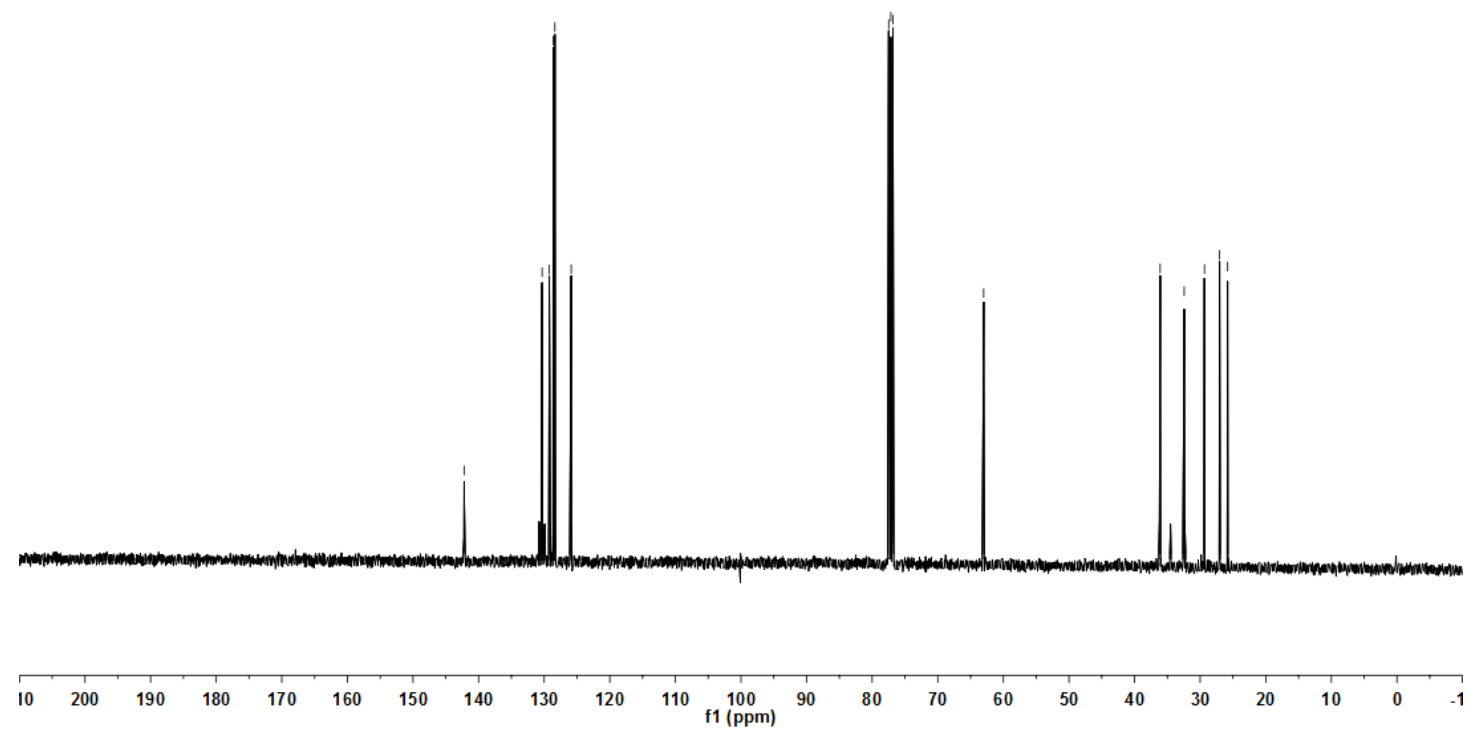


${ }^{1} \mathrm{H}$ NMR (400 MHz, $\mathrm{CDCl}_{3}$ ) and ${ }^{13} \mathrm{C}$ NMR (101 MHz, $\left.\mathrm{CDCl}_{3}\right)$ of $4 \mathrm{a}$

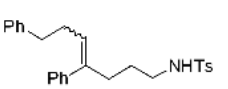

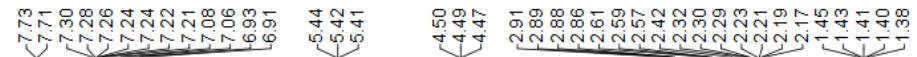
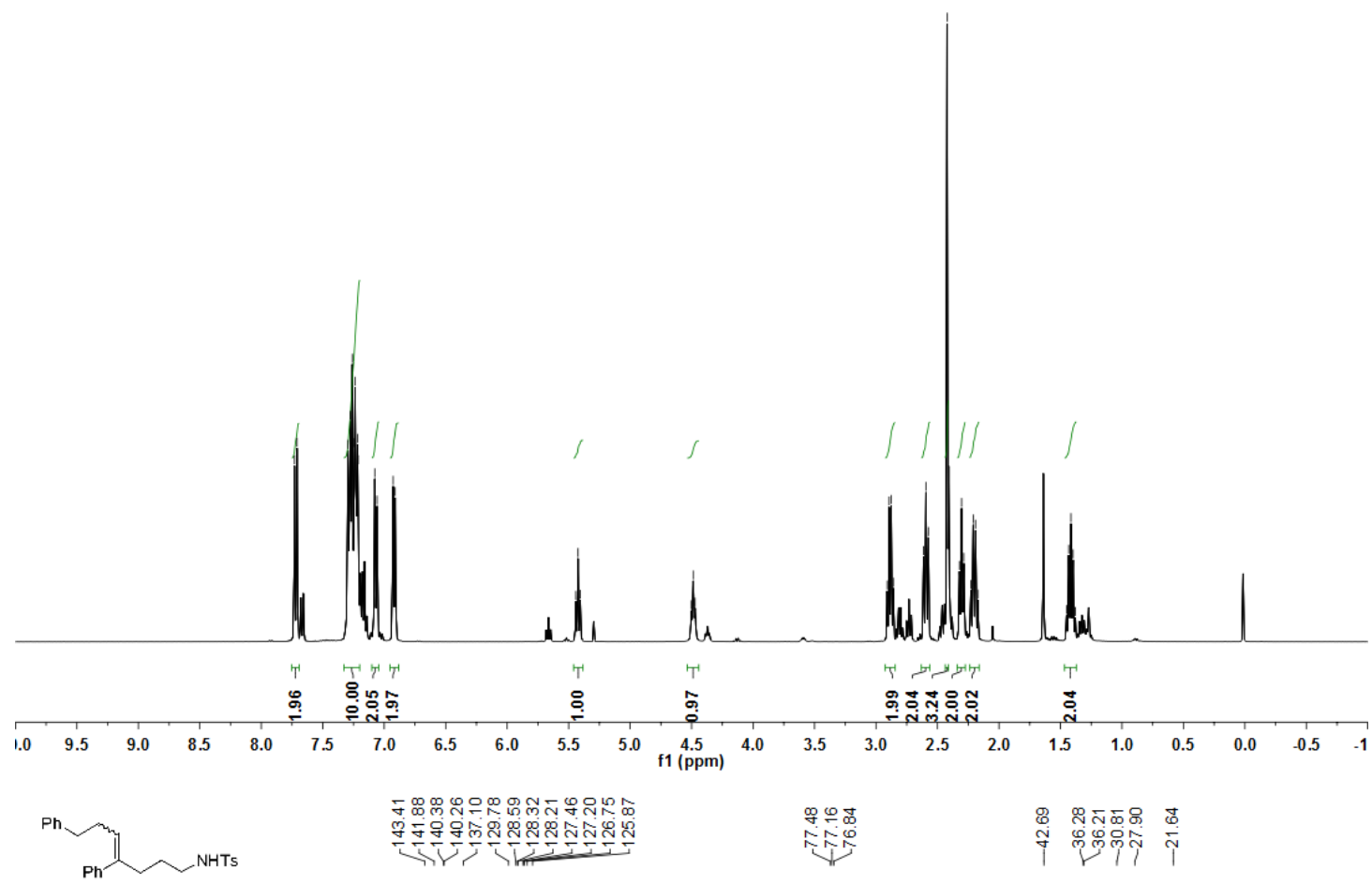

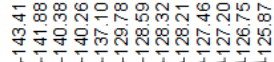

诲篎

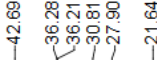

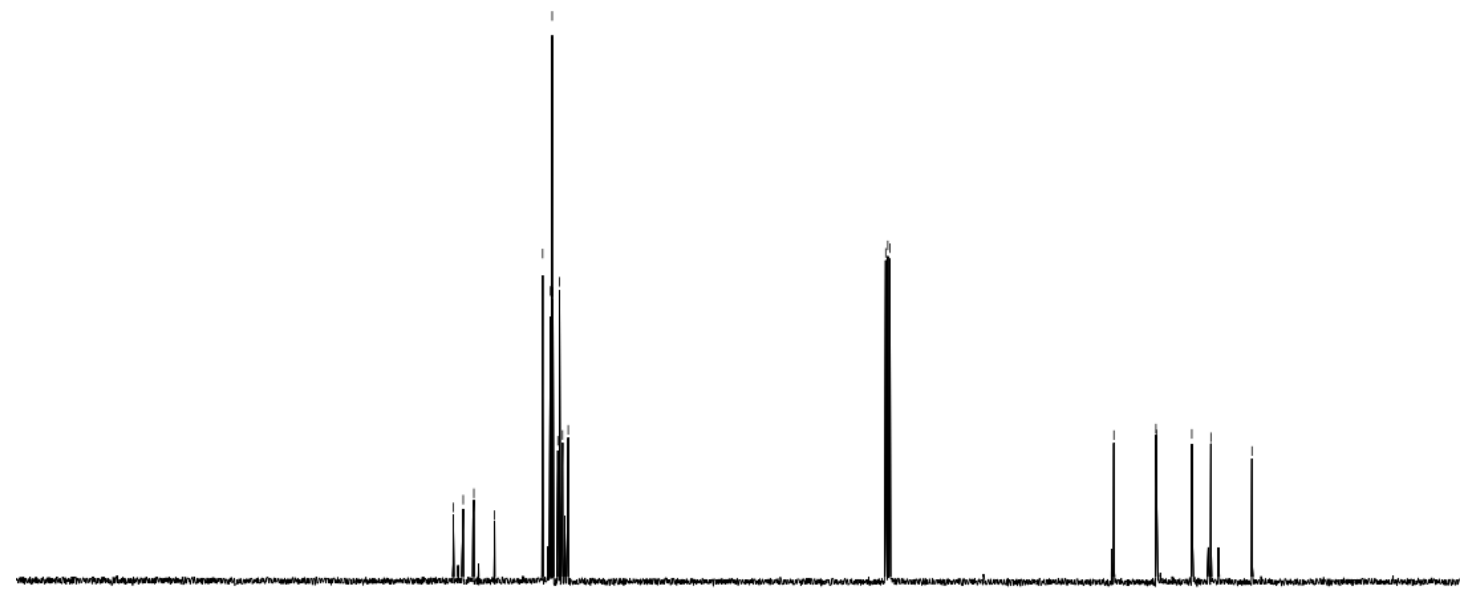

\begin{tabular}{llllllllllllllllllllllll}
\hline 10 & 200 & 190 & 180 & 170 & 160 & 150 & 140 & 130 & 120 & 110 & $\begin{array}{c}100 \\
\mathrm{f} 1(\mathrm{ppm})\end{array}$ & 90 & 80 & 70 & 60 & 50 & 40 & 30 & 20 & 10 & 0 & -1
\end{tabular} 
${ }^{1} \mathrm{H}$ NMR (400 MHz, $\left.\mathrm{CDCl}_{3}\right)$ and ${ }^{13} \mathrm{C}$ NMR (101 $\left.\mathrm{MHz}, \mathrm{CDCl}_{3}\right)$ of 4b
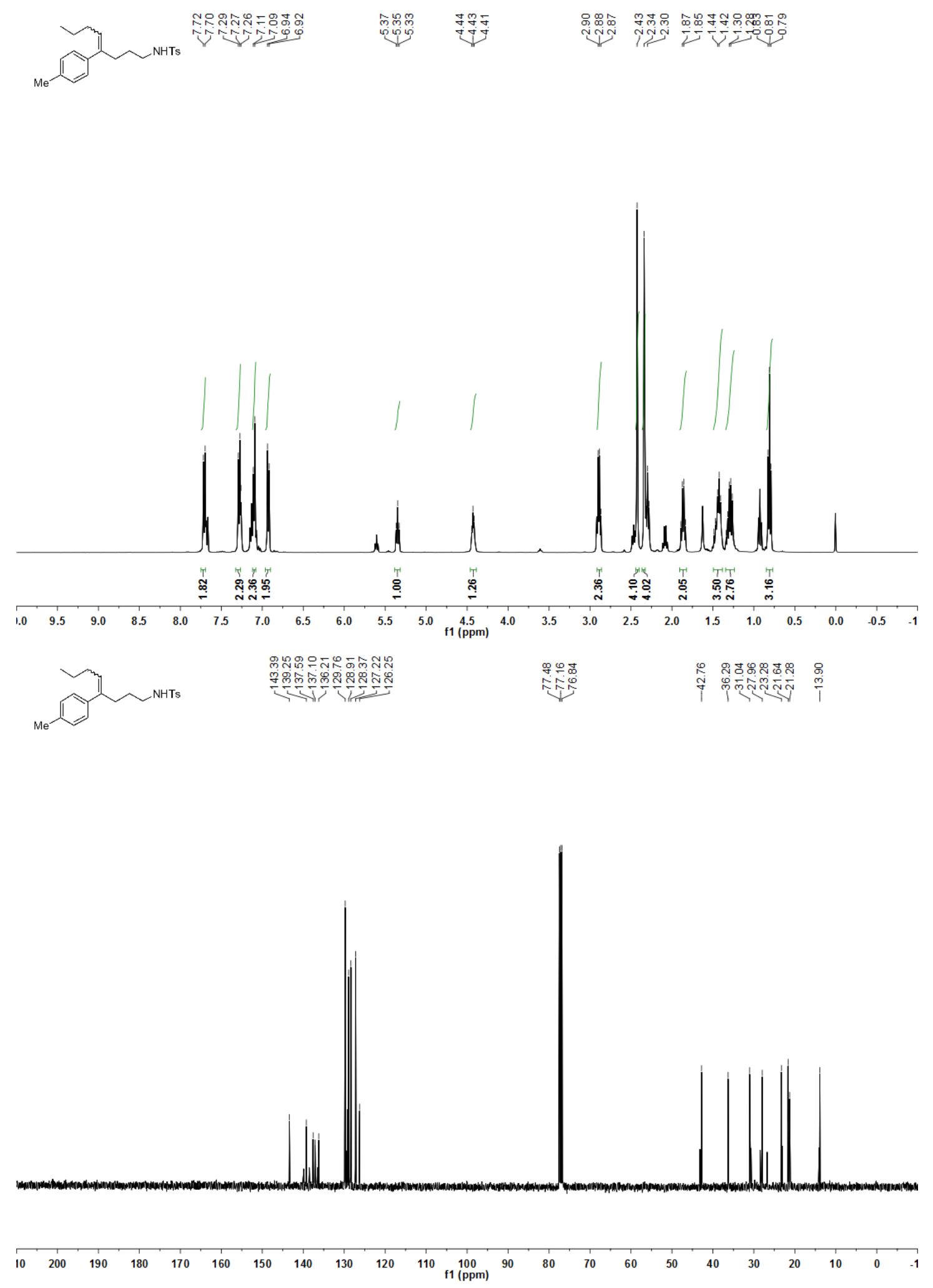
${ }^{1} \mathrm{H}$ NMR (400 MHz, $\left.\mathrm{CDCl}_{3}\right)$ and ${ }^{13} \mathrm{C}$ NMR (101 MHz, $\left.\mathrm{CDCl}_{3}\right)$ of $4 \mathrm{c}$
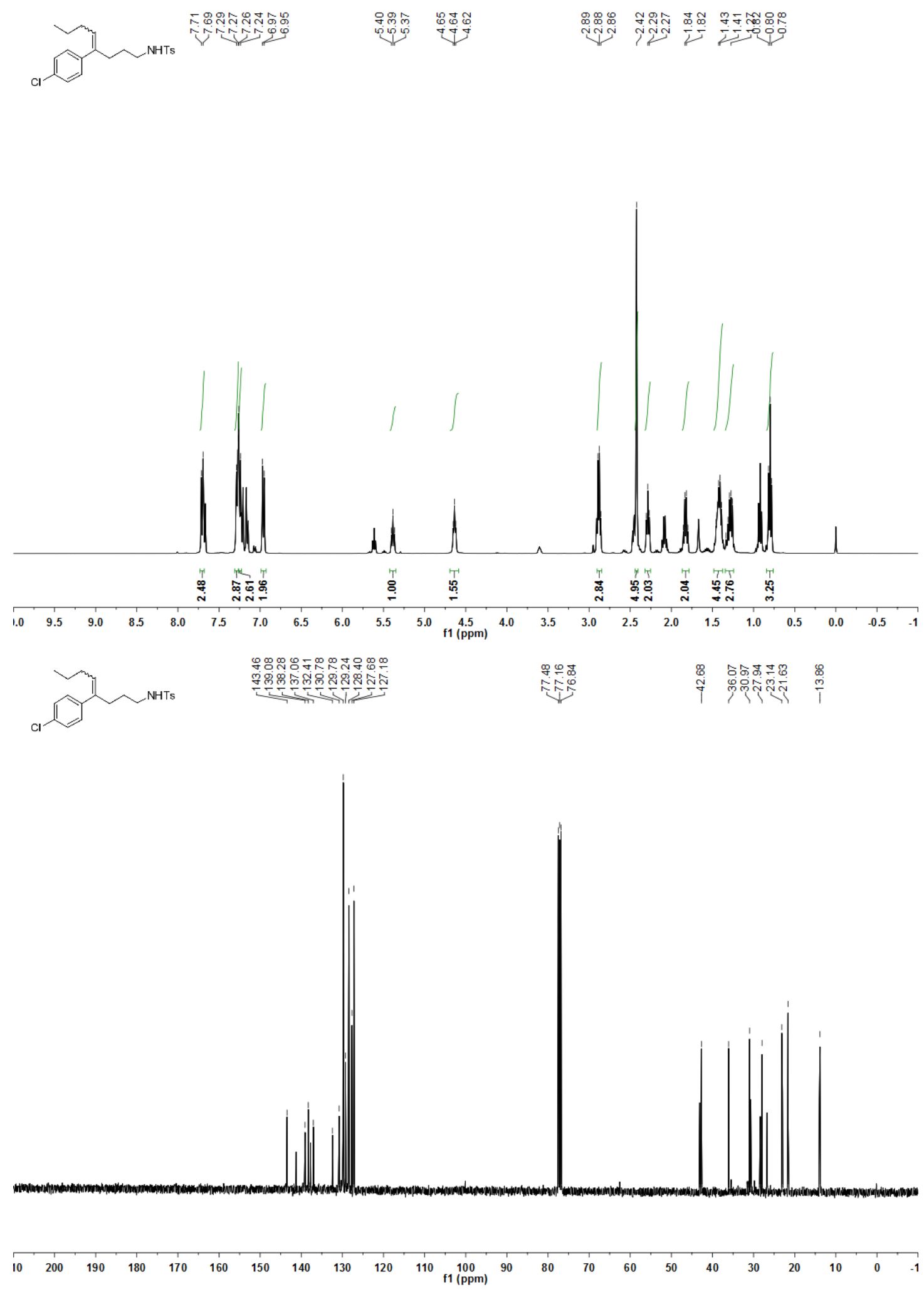
${ }^{1} \mathrm{H}$ NMR (400 MHz, $\left.\mathrm{CDCl}_{3}\right)$ and ${ }^{13} \mathrm{C}$ NMR $\left(101 \mathrm{MHz}, \mathrm{CDCl}_{3}\right)$ of $4 \mathrm{~d}$

Ph⿻上丨冖

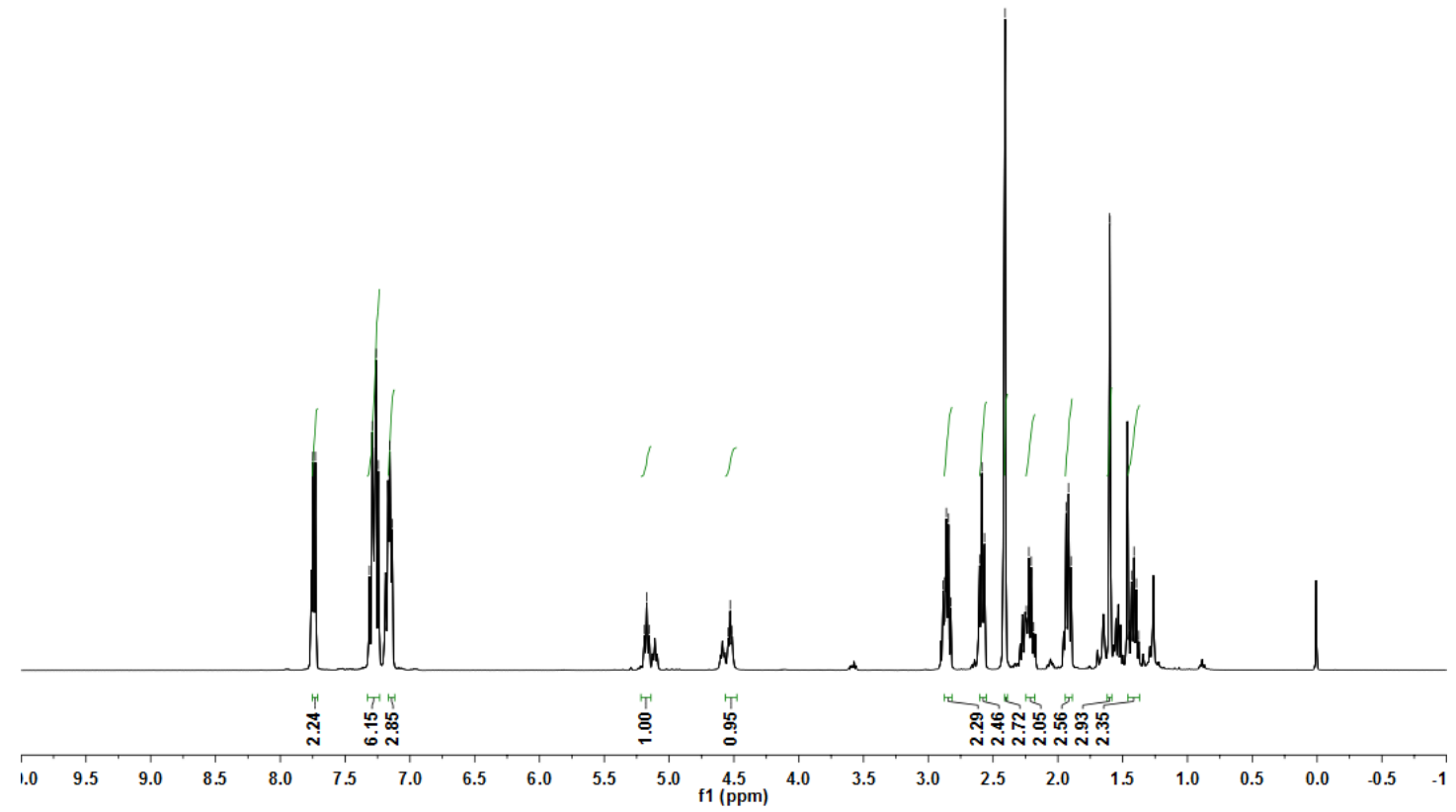

$\underbrace{\text { Ph丩m }}_{\text {NHTs }}$

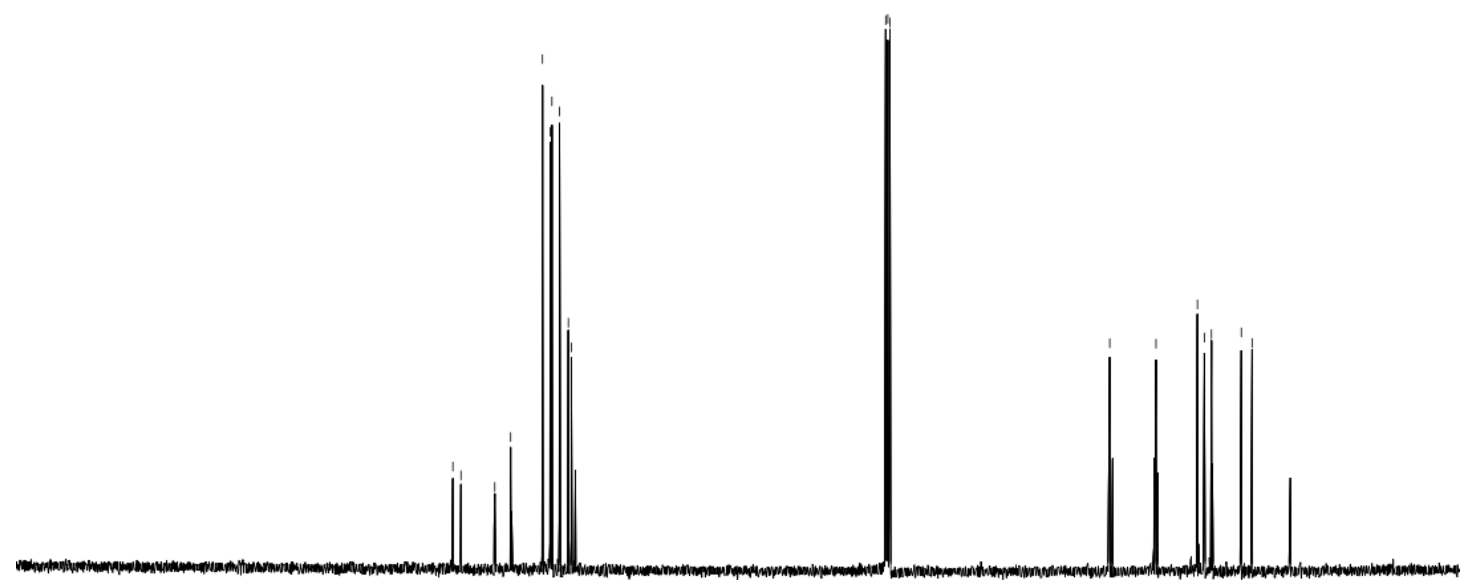

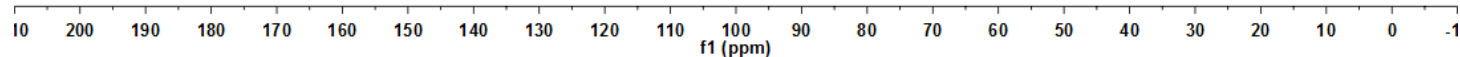


${ }^{1} \mathrm{H}$ NMR (400 MHz, $\left.\mathrm{CDCl}_{3}\right)$ and ${ }^{13} \mathrm{C}$ NMR (101 $\left.\mathrm{MHz}, \mathrm{CDCl}_{3}\right)$ of $4 \mathrm{e}$ 等

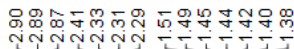
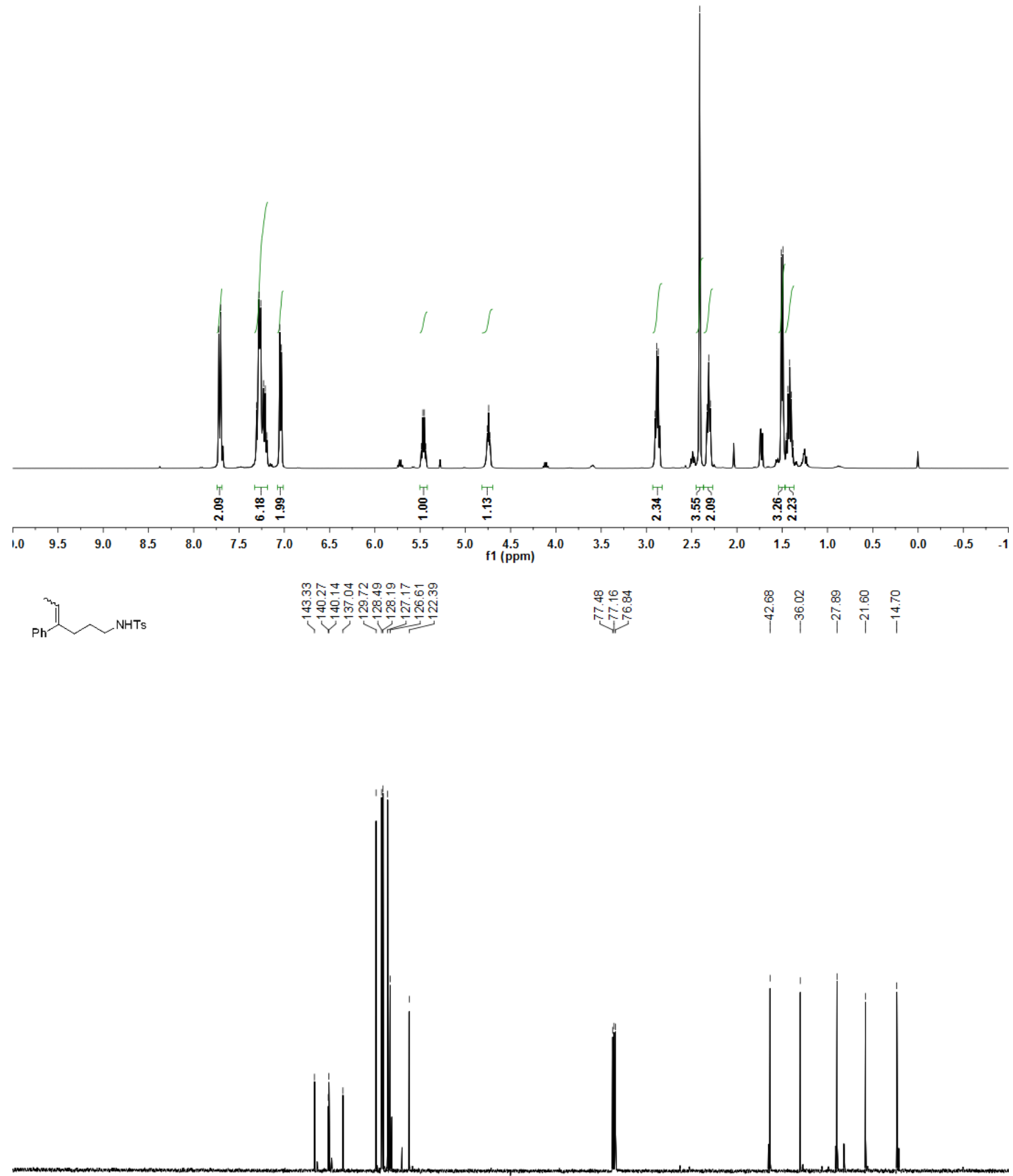

\begin{tabular}{|llllllllllllllllllllllll}
\hline 10 & 200 & 190 & 180 & 170 & 160 & 150 & 140 & 130 & 120 & 110 & 100 & 90 & 80 & 70 & 60 & 50 & 40 & 30 & 20 & 10 & 0 & -1
\end{tabular} 
${ }^{1} \mathrm{H}$ NMR (400 MHz, $\left.\mathrm{CDCl}_{3}\right)$ and ${ }^{13} \mathrm{C}$ NMR (101 MHz, $\left.\mathrm{CDCl}_{3}\right)$ of $4 \mathrm{f}$

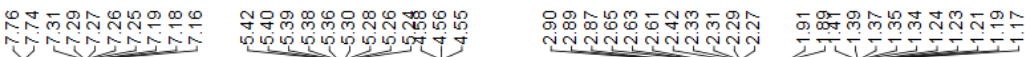

$\mathrm{Ph}_{\mathrm{NHTS}}$

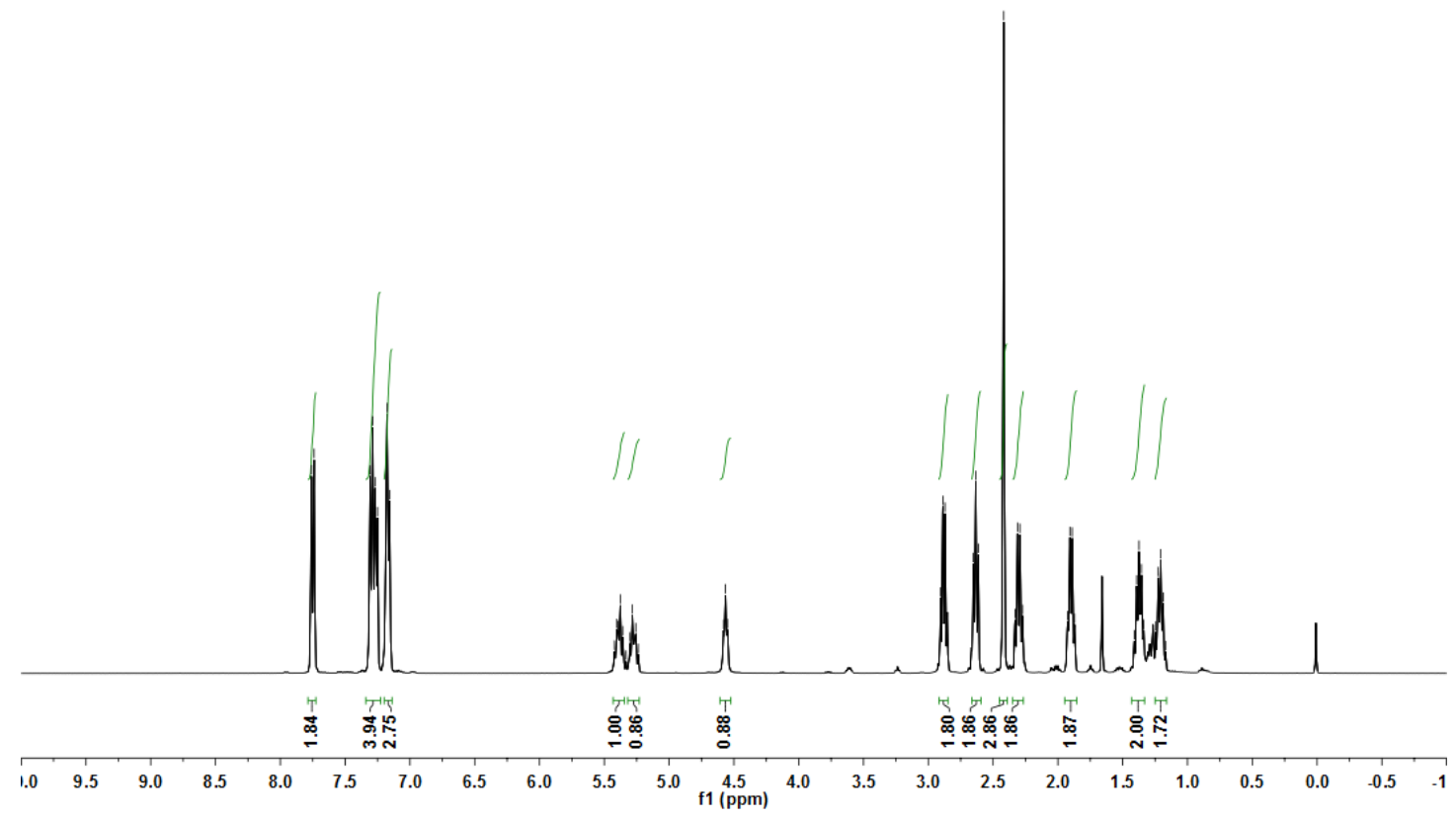

Ph—un NHTs

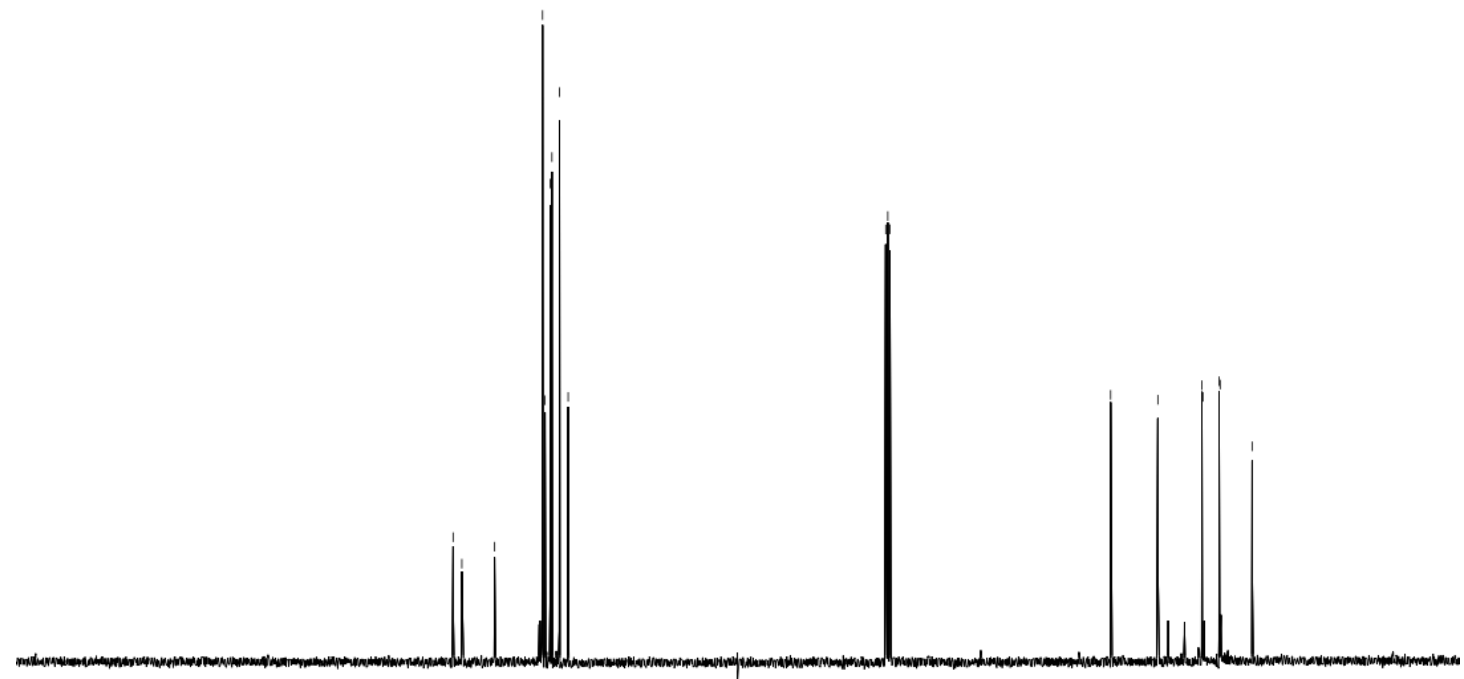

$\begin{array}{lllllllllllllllllllllllllllllll}10 & 200 & 190 & 180 & 170 & 160 & 150 & 140 & 130 & 120 & 110 & \begin{array}{c}100 \\ \mathrm{f} 1(\mathrm{ppm})\end{array} & 90 & 80 & 70 & 60 & 50 & 40 & 30 & 20 & 10 & 0 & -1\end{array}$ 
${ }^{1} \mathrm{H}$ NMR (400 MHz, $\left.\mathrm{CDCl}_{3}\right)$ and ${ }^{13} \mathrm{C}$ NMR (101 MHz, $\left.\mathrm{CDCl}_{3}\right)$ of $2 \mathrm{a}$

$\overbrace{\mathrm{Ph}} \mathrm{Ph}_{\mathrm{ph}}$

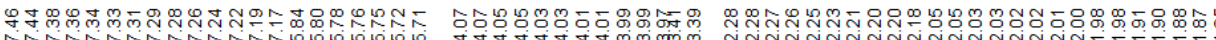

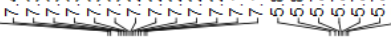

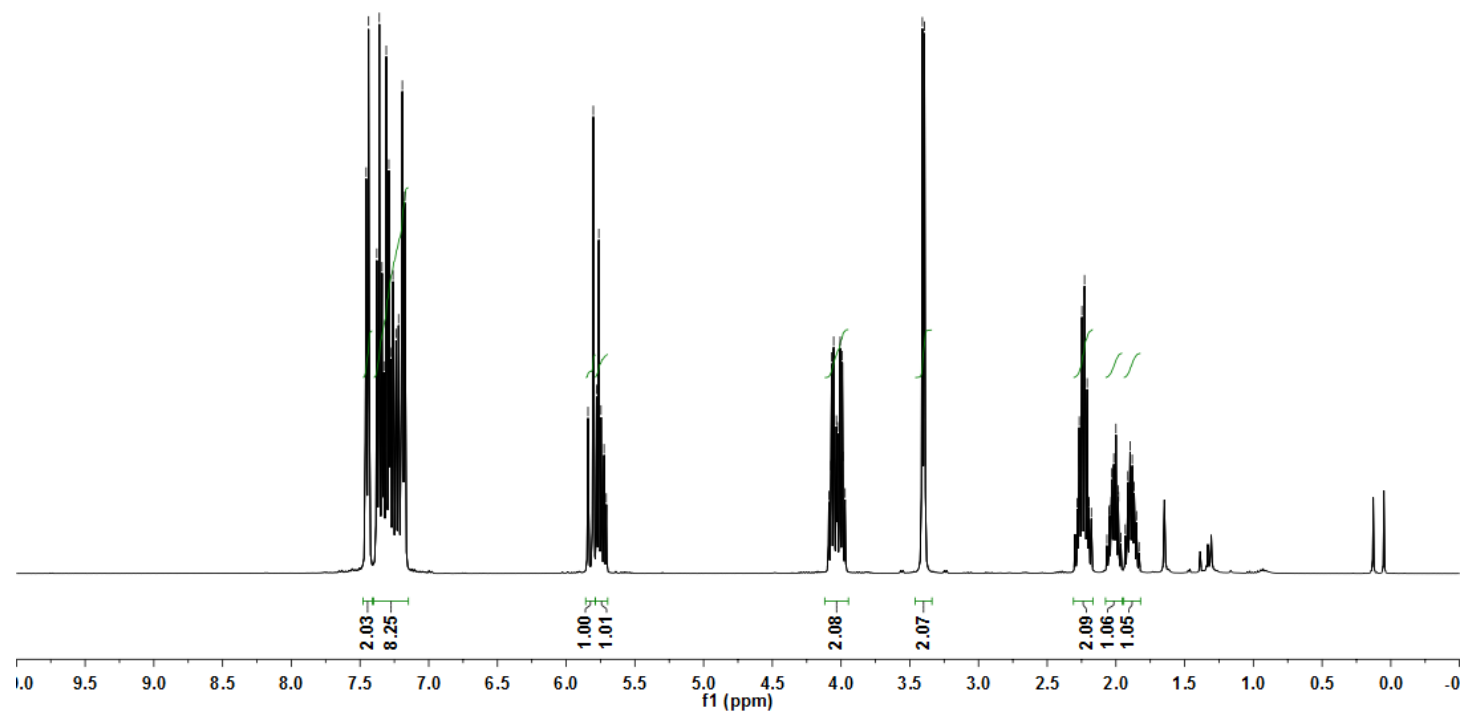

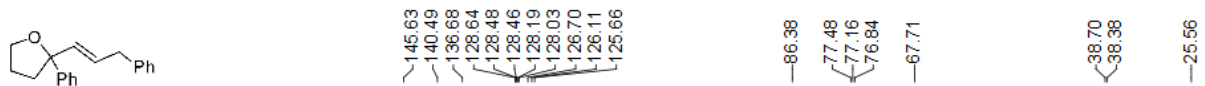

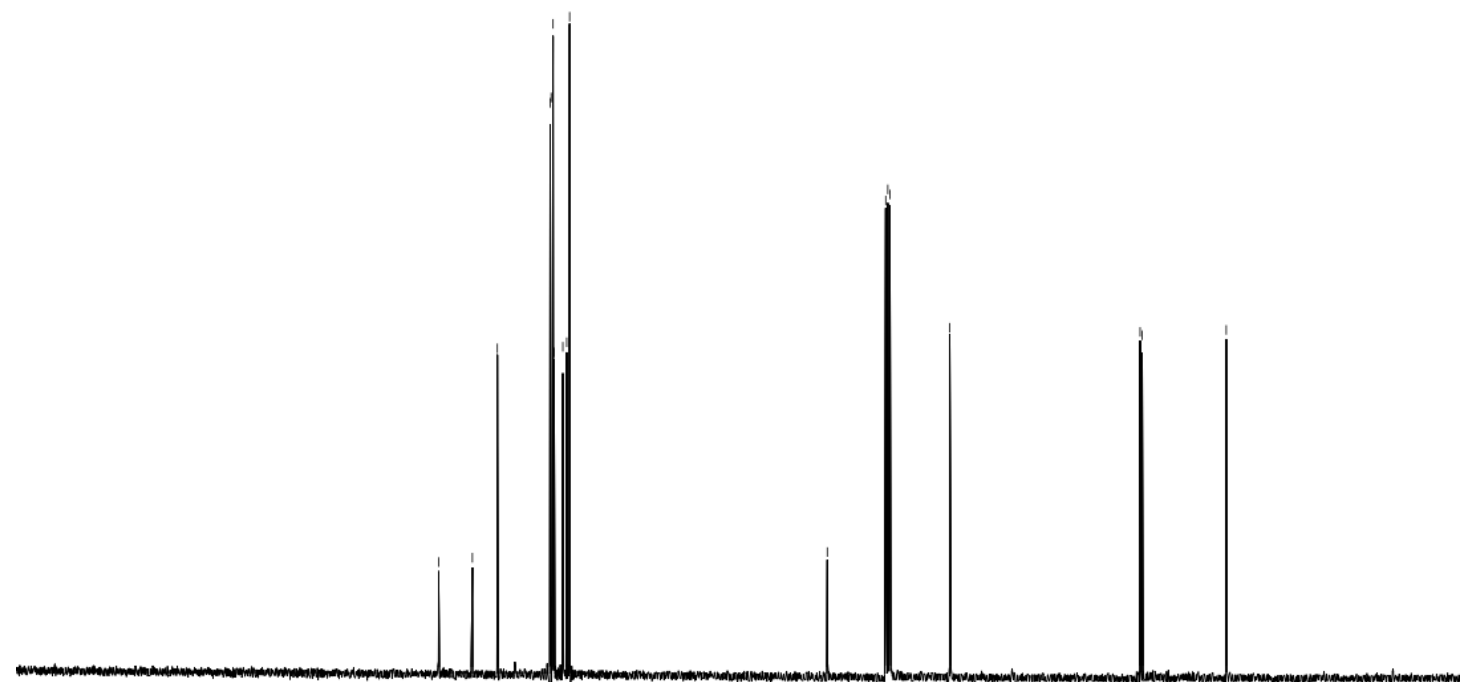

\begin{tabular}{|llllllllllllllllllllllll}
\hline 10 & 200 & 190 & 180 & 170 & 160 & 150 & 140 & 130 & 120 & 110 & 100 & 90 & 80 & 70 & 60 & 50 & 40 & 30 & 20 & 10 & 0 & -1
\end{tabular} 
${ }^{1} \mathrm{H}$ NMR (400 MHz, $\left.\mathrm{CDCl}_{3}\right)$ and ${ }^{13} \mathrm{C}$ NMR (101 MHz, $\left.\mathrm{CDCl}_{3}\right)$ of $2 \mathrm{~b}$

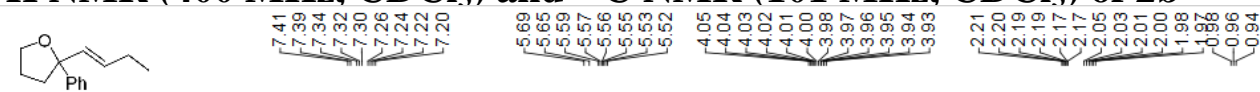

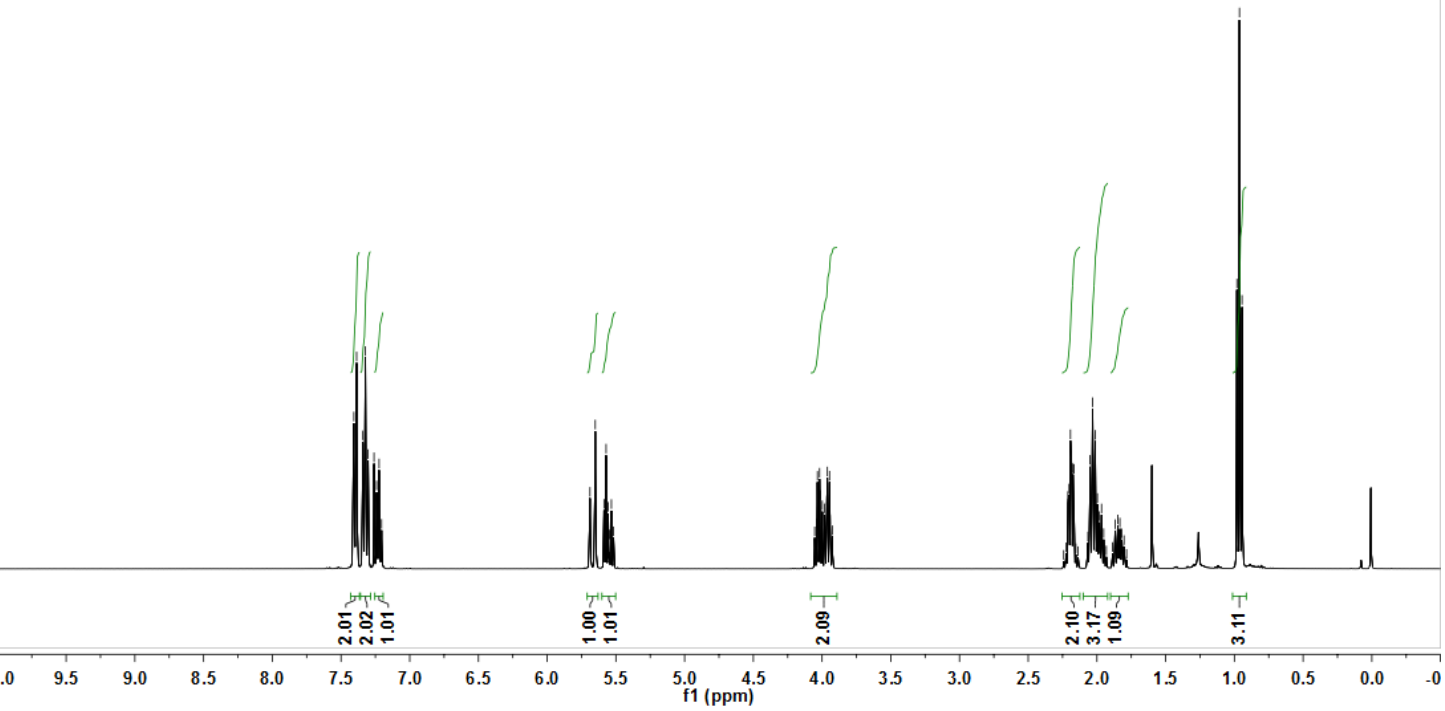

[0 ר

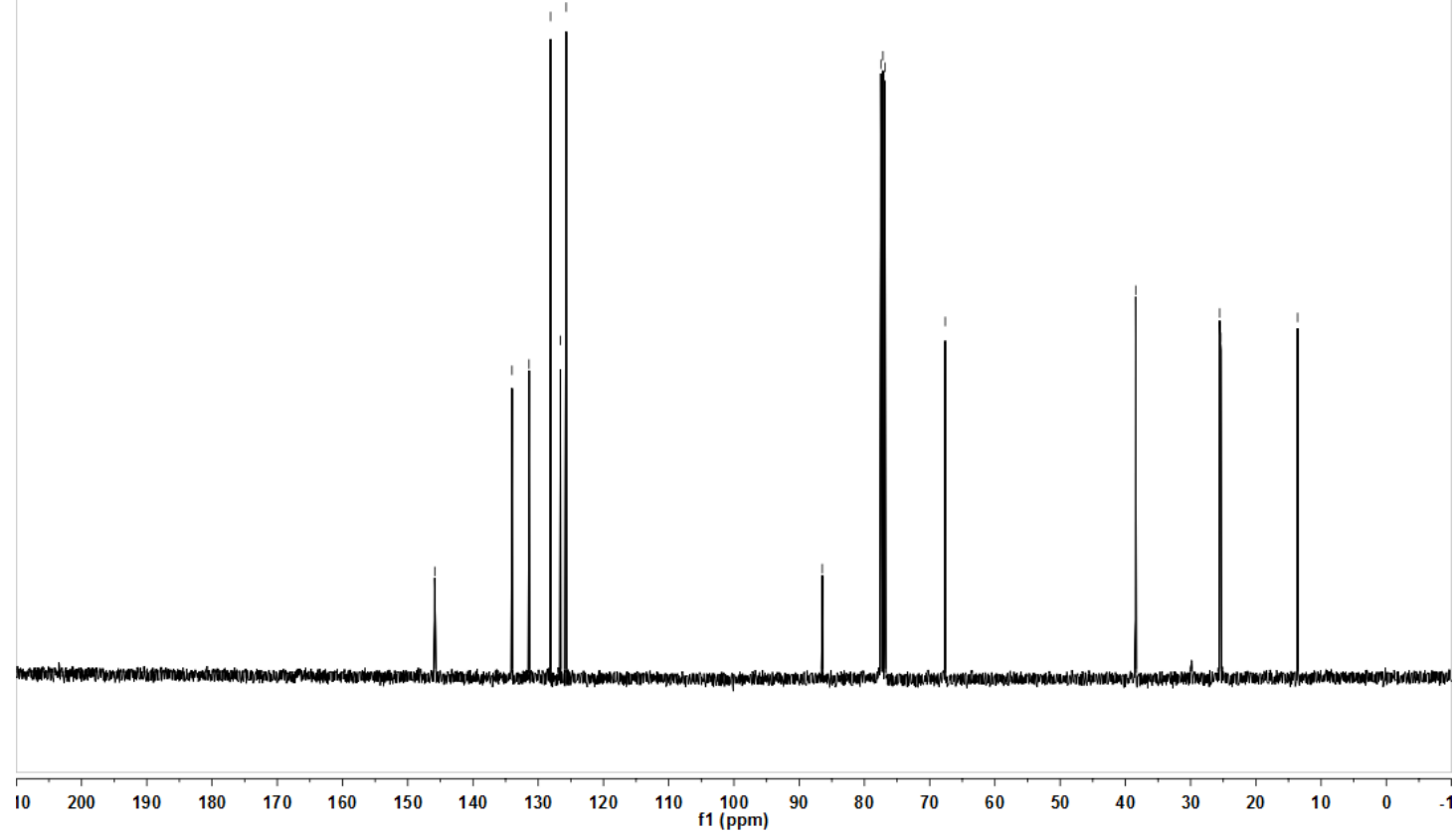


${ }^{1} \mathrm{H}$ NMR (400 MHz, $\left.\mathrm{CDCl}_{3}\right)$ and ${ }^{13} \mathrm{C}$ NMR (101 MHz, $\left.\mathrm{CDCl}_{3}\right)$ of $2 \mathrm{c}$

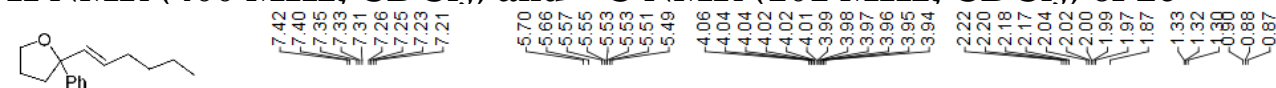
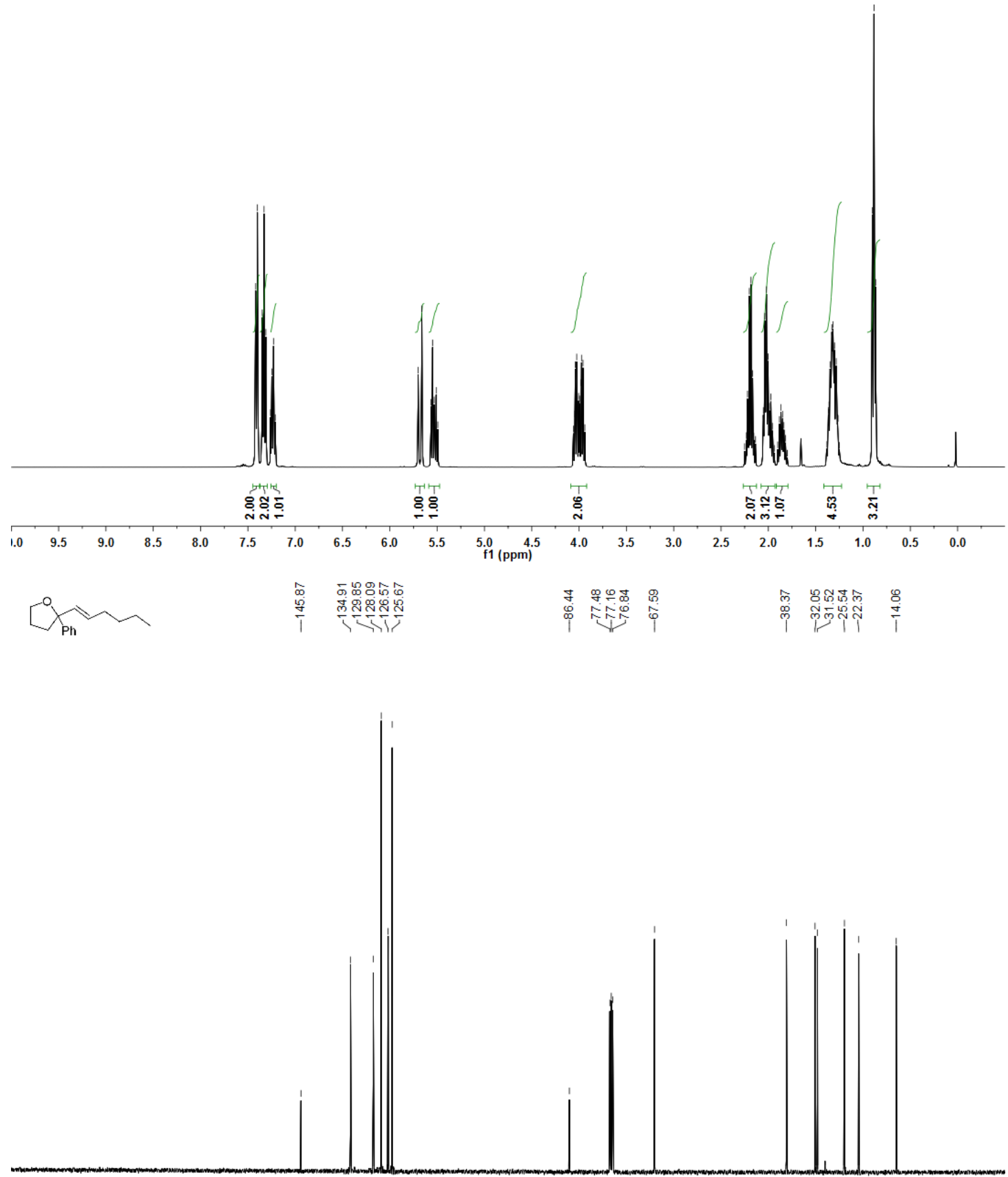

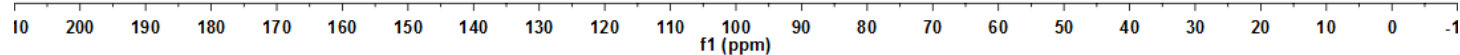


${ }^{1} \mathrm{H}$ NMR (400 MHz, $\left.\mathrm{CDCl}_{3}\right)$ and ${ }^{13} \mathrm{C}$ NMR (101 MHz, $\left.\mathrm{CDCl}_{3}\right)$ of $2 \mathrm{~d}$

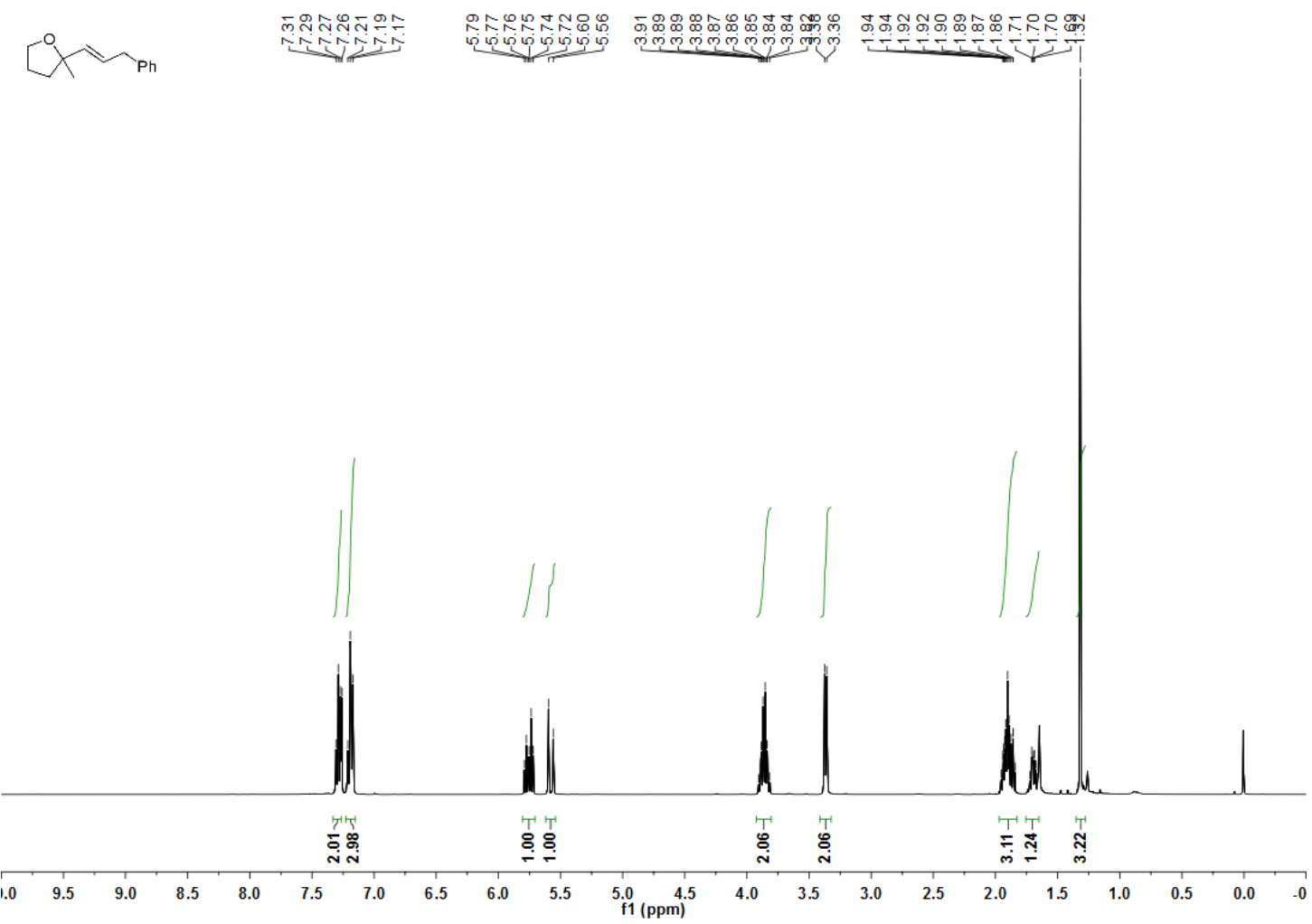

[X⿻一亅八

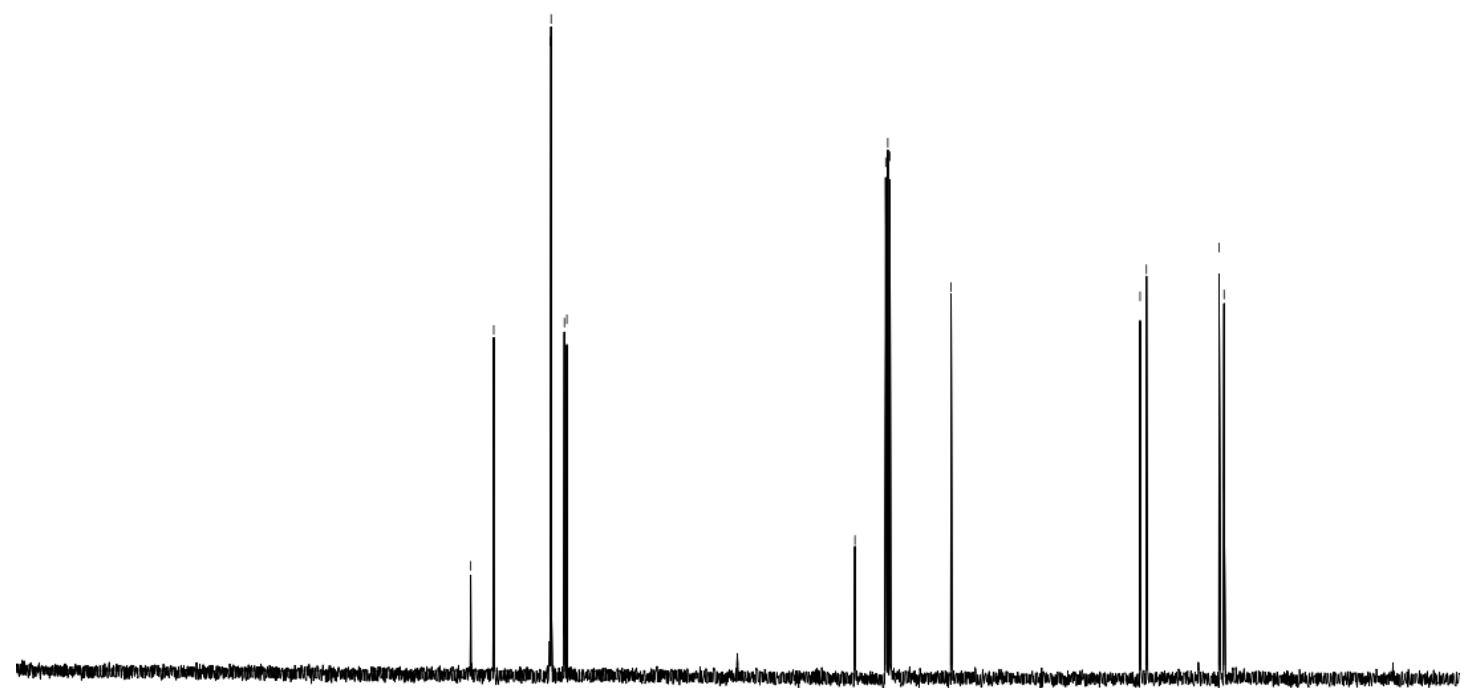

$\begin{array}{llllllllllllllllllllllllllllllllll}10 & 200 & 190 & 180 & 170 & 160 & 150 & 140 & 130 & 120 & 110 & \begin{array}{c}100 \\ \mathrm{f} 1(\mathrm{ppm})\end{array} & 90 & 80 & 70 & 60 & 50 & 40 & 30 & 20 & 10 & 0 & -1\end{array}$ 
${ }^{1} \mathrm{H}$ NMR (400 MHz, $\left.\mathrm{CDCl}_{3}\right)$ and ${ }^{13} \mathrm{C}$ NMR (101 MHz, $\left.\mathrm{CDCl}_{3}\right)$ of $2 \mathrm{e}$
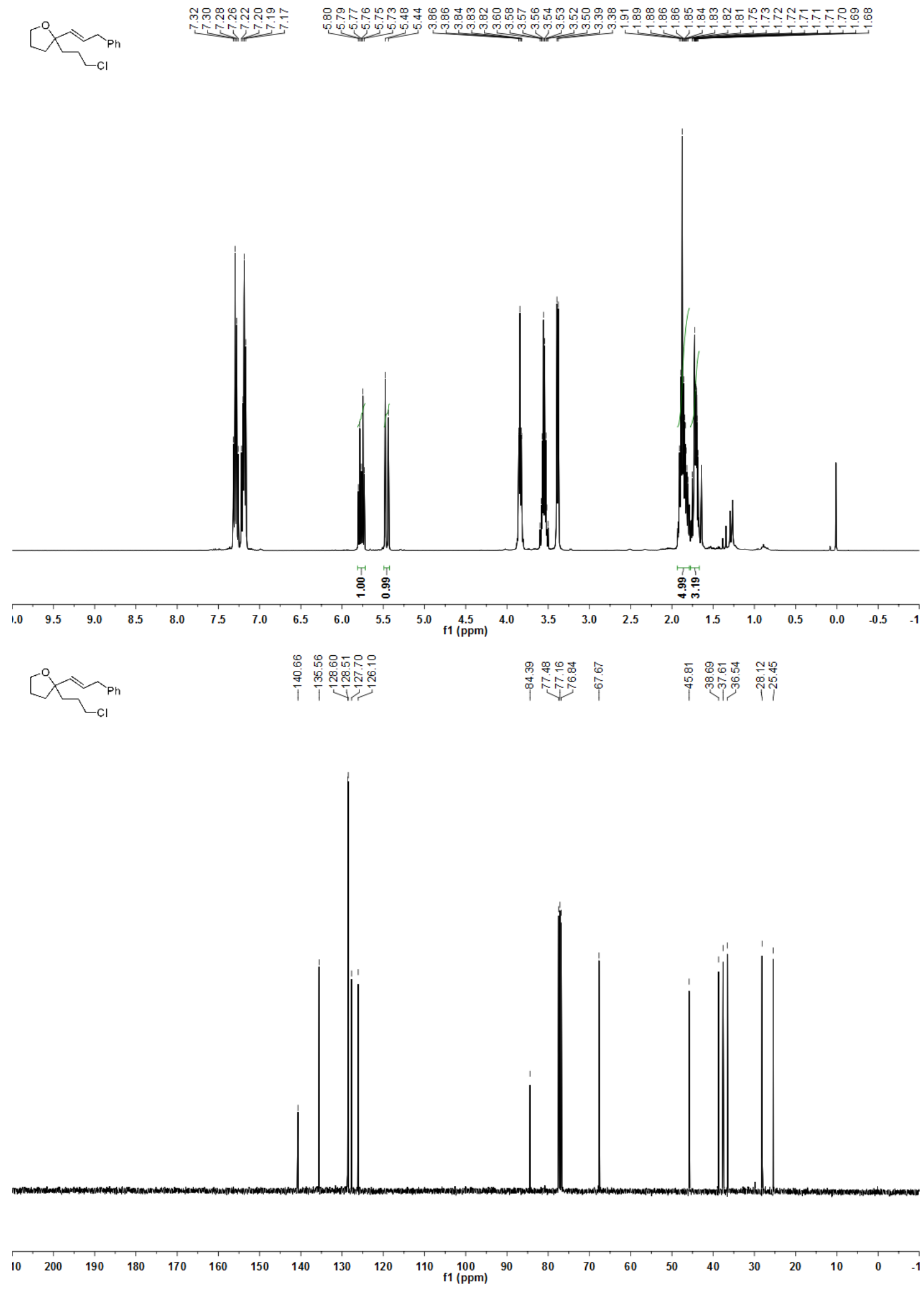
${ }^{1} \mathrm{H}$ NMR (400 MHz, $\left.\mathrm{CDCl}_{3}\right)$ and $\left.{ }^{13} \mathrm{C} \mathrm{NMR} \mathrm{(101} \mathrm{MHz,} \mathrm{CDCl}_{3}\right)$ of $2 \mathrm{f}$

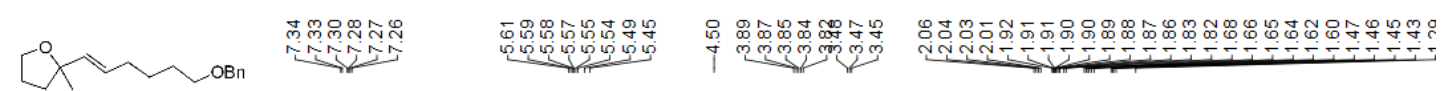
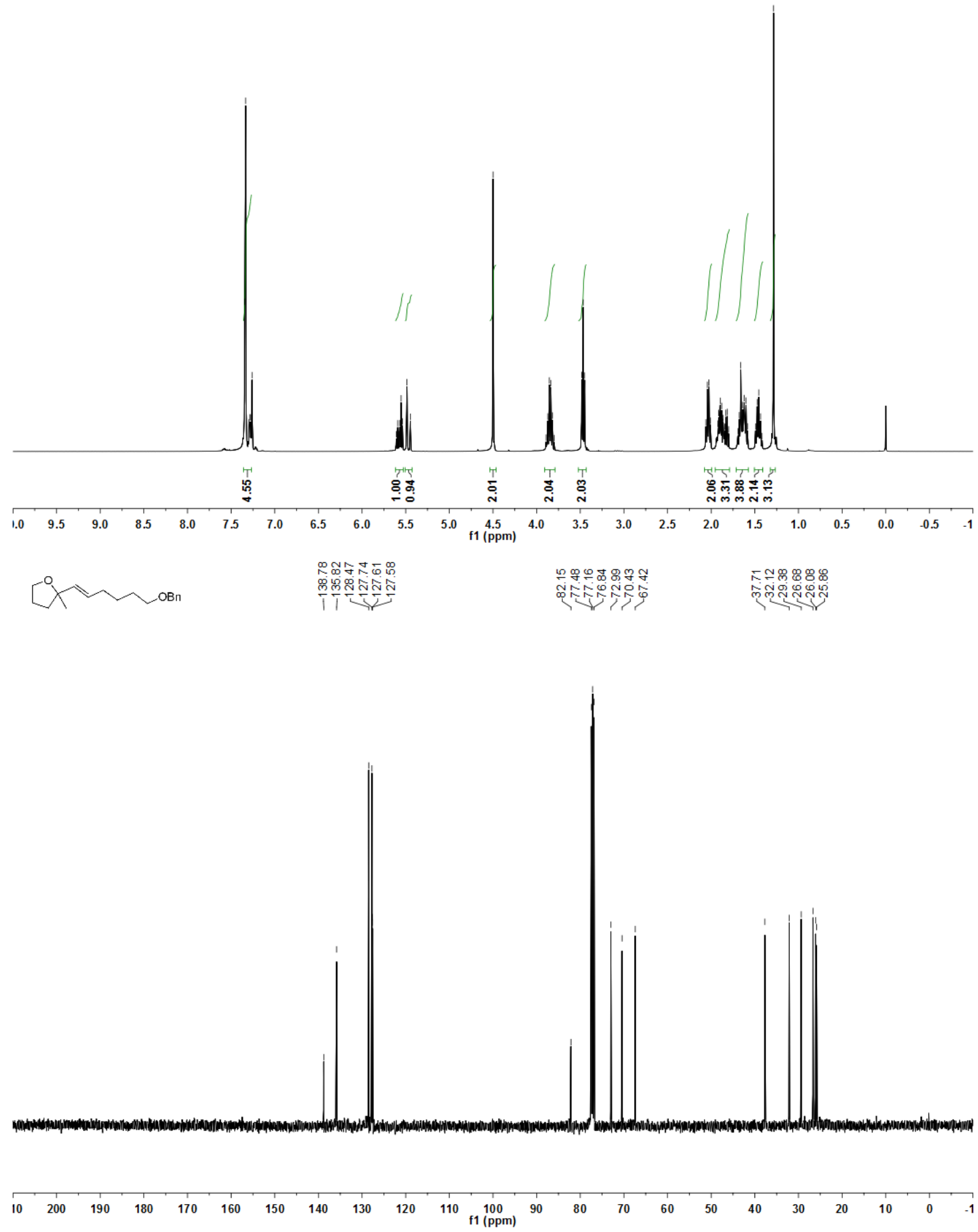
${ }^{1} \mathrm{H}$ NMR (400 MHz, $\left.\mathrm{CDCl}_{3}\right)$ and ${ }^{13} \mathrm{C}$ NMR (101 MHz, $\left.\mathrm{CDCl}_{3}\right)$ of $2 \mathrm{~g}$
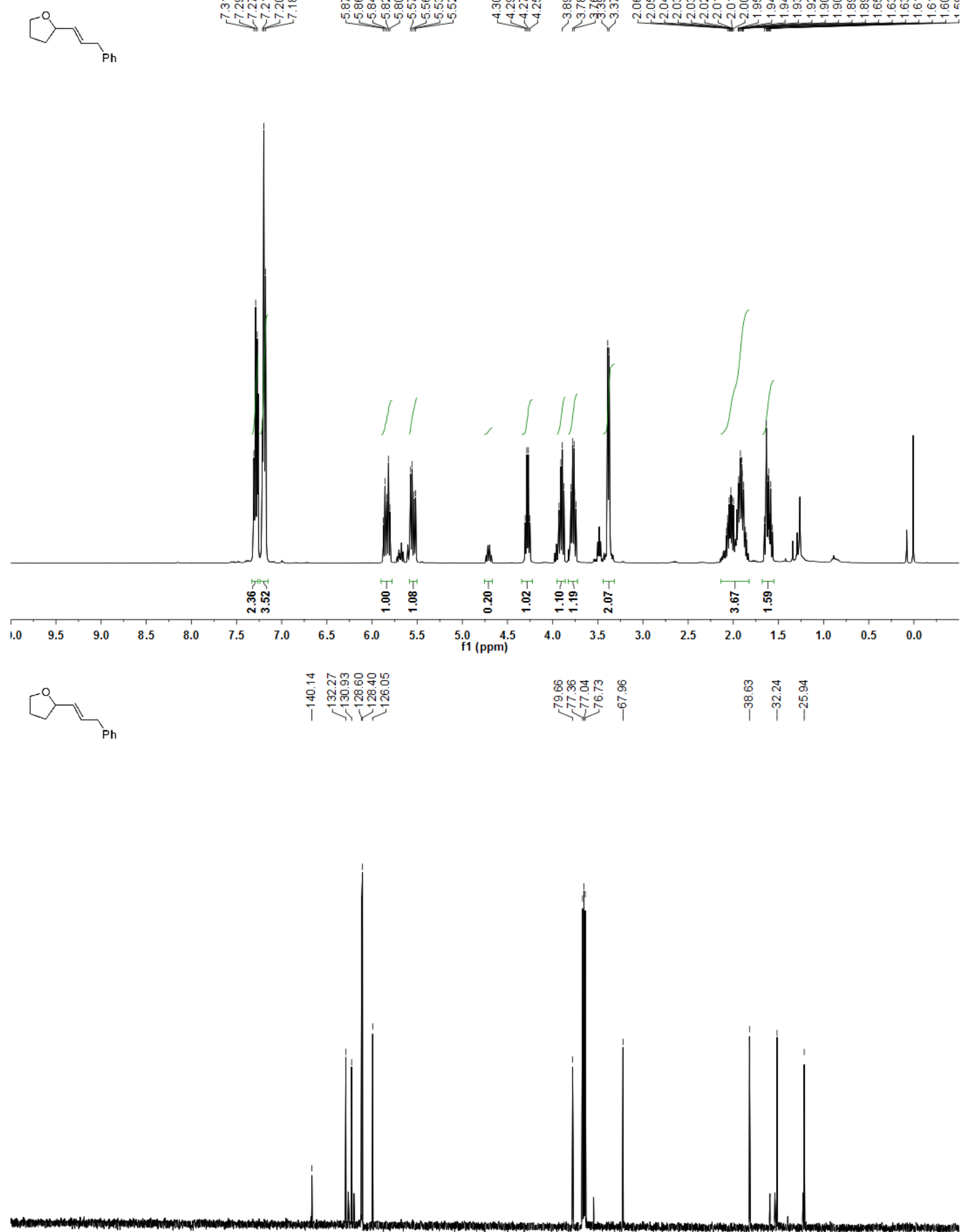

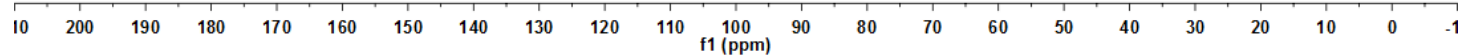


${ }^{1} \mathrm{H}$ NMR (400 MHz, $\left.\mathrm{CDCl}_{3}\right)$ and ${ }^{13} \mathrm{C}$ NMR (101 MHz, $\left.\mathrm{CDCl}_{3}\right)$ of $2 \mathrm{~h}$<smiles>[C]1CC2CCCC(C1)C2</smiles>
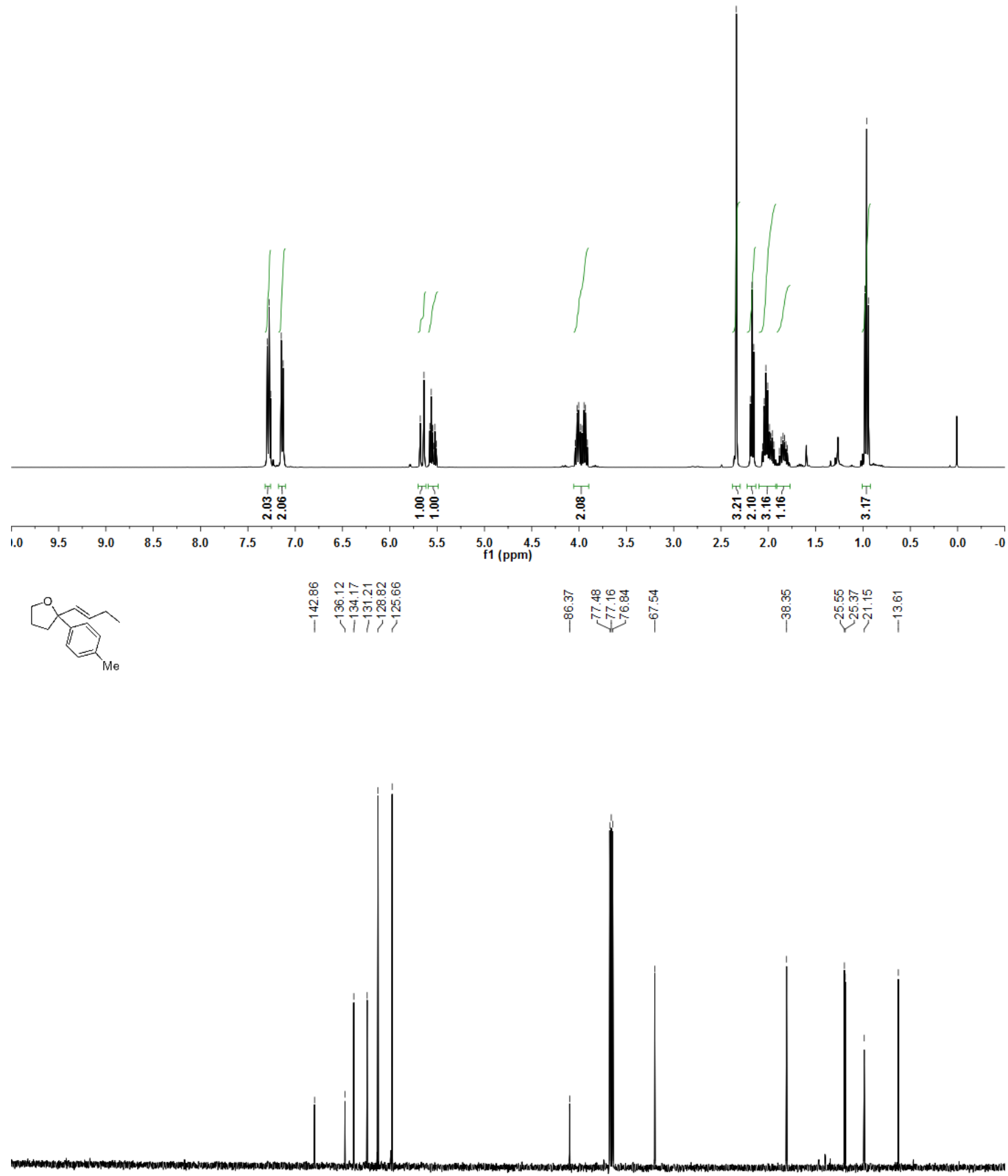

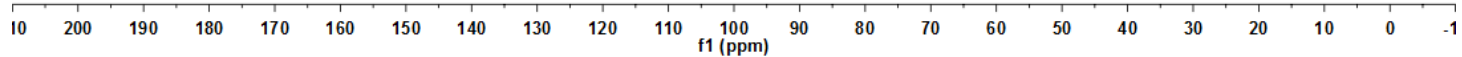


${ }^{1} \mathrm{H}$ NMR (400 MHz, $\left.\mathrm{CDCl}_{3}\right)$ and ${ }^{13} \mathrm{C}$ NMR (101 MHz, $\left.\mathrm{CDCl}_{3}\right)$ of $2 \mathrm{i}$

[Y-me

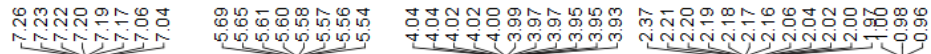
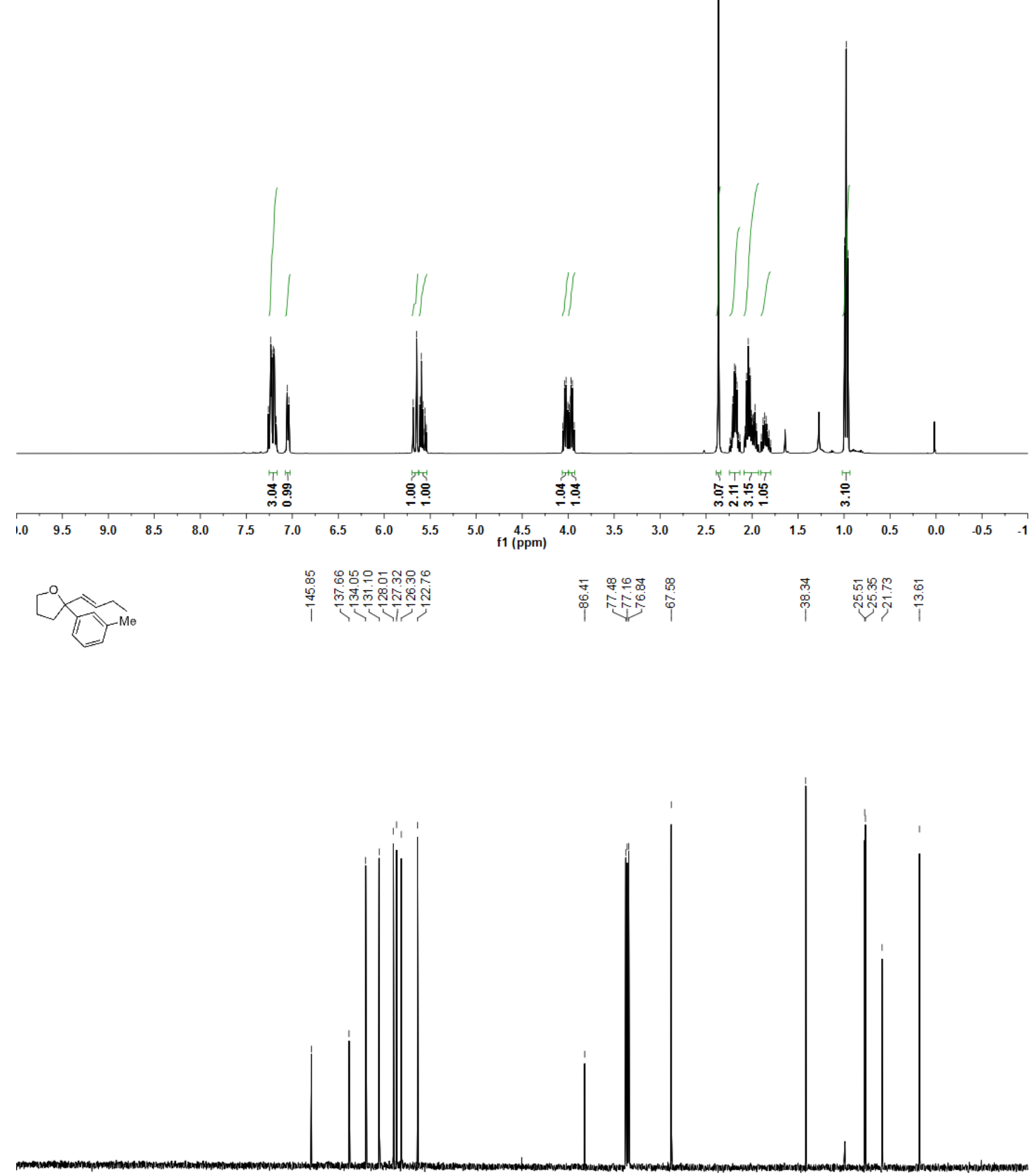

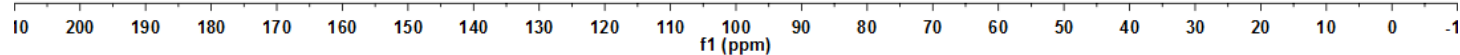


${ }^{1} \mathrm{H}$ NMR $\left(400 \mathrm{MHz}, \mathrm{CDCl}_{3}\right)$ and $\left.{ }^{13} \mathrm{C} \mathrm{NMR} \mathrm{(101} \mathrm{MHz,} \mathrm{CDCl}_{3}\right)$ of $2 \mathrm{i}$

$\mathbb{R}_{i \mathrm{rr}}$

N
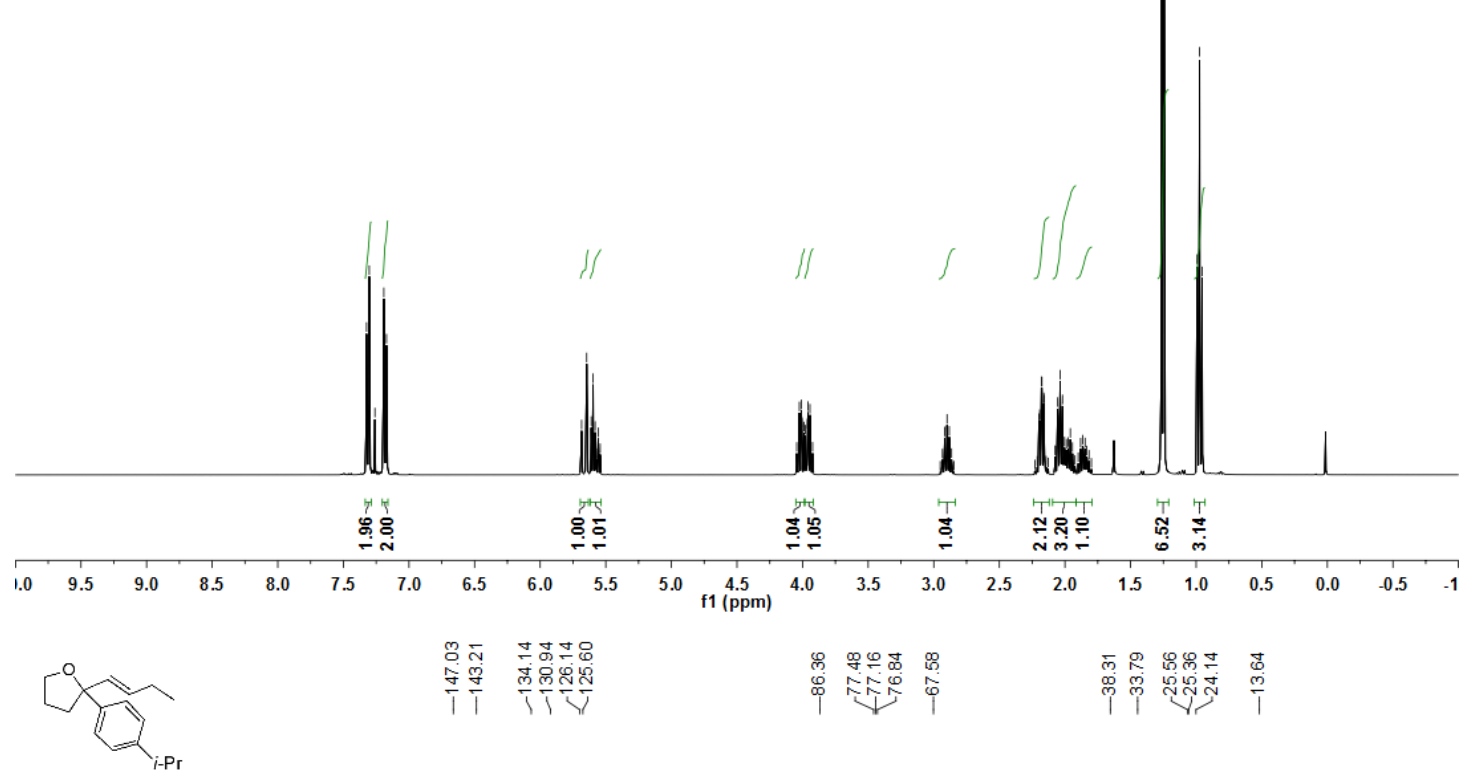

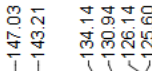

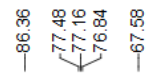

iٓj

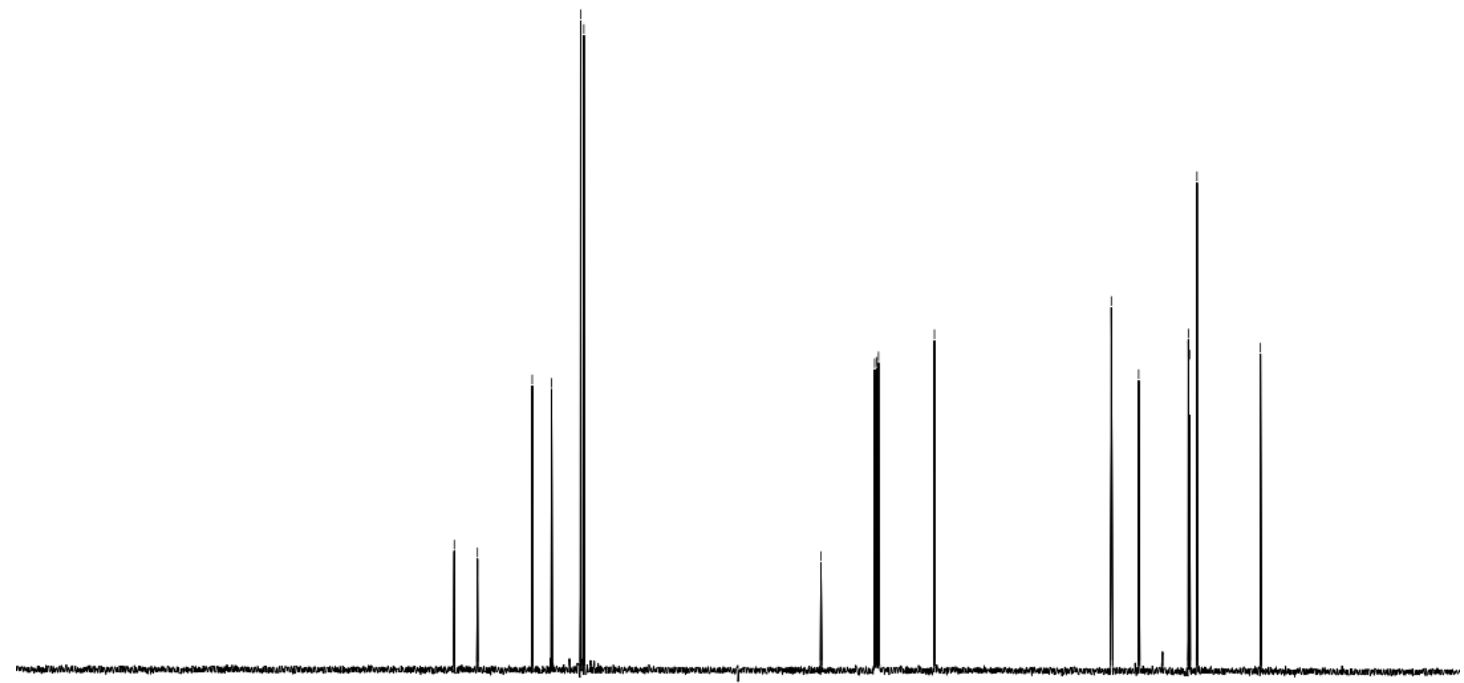

$\begin{array}{llllllllllllllllllllllllllll}1 & 200 & 190 & 180 & 170 & 160 & 150 & 140 & 130 & 120 & 110 & \begin{array}{l}100 \\ \mathrm{f} 1(\mathrm{ppm})\end{array} & 90 & 80 & 70 & 60 & 50 & 40 & 30 & 20 & 10 & 0 & -10\end{array}$ 
${ }^{1} \mathrm{H}$ NMR (400 MHz, $\left.\mathrm{CDCl}_{3}\right)$ and ${ }^{13} \mathrm{C}$ NMR (101 MHz, $\left.\mathrm{CDCl}_{3}\right)$ of $2 \mathrm{k}$

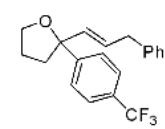

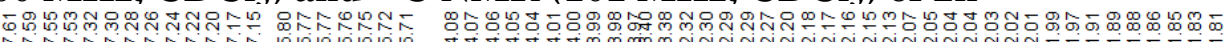
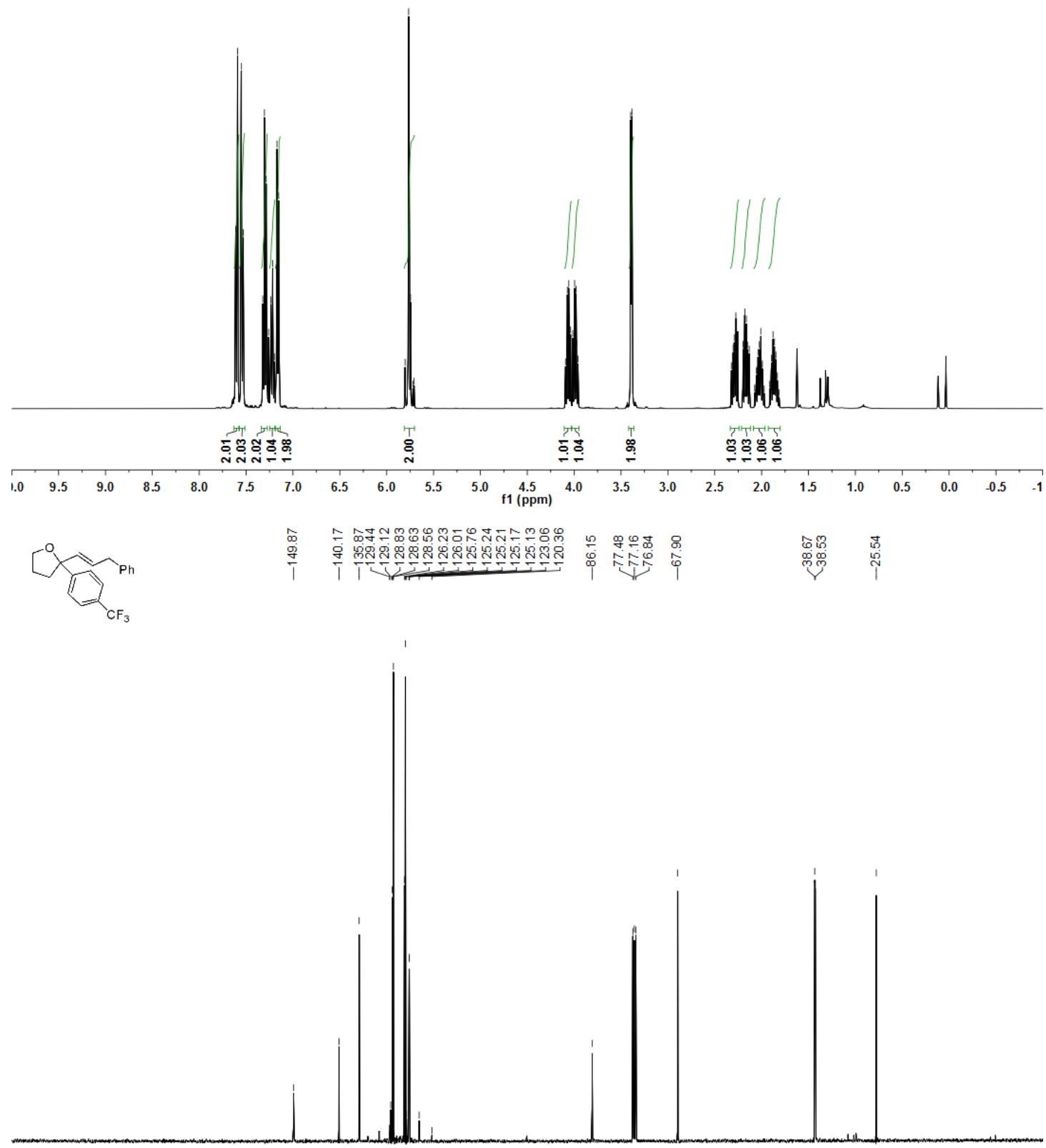

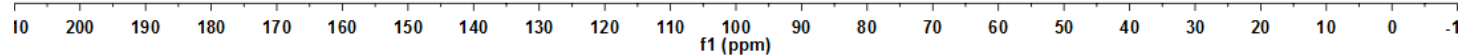


${ }^{1} \mathrm{H}$ NMR (400 MHz, $\left.\mathrm{CDCl}_{3}\right)$ and ${ }^{13} \mathrm{C}$ NMR (101 MHz, $\left.\mathrm{CDCl}_{3}\right)$ of $2 \mathrm{l}$

$\mathbb{1} x_{c 1}$

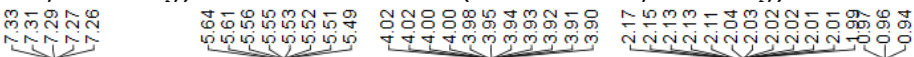
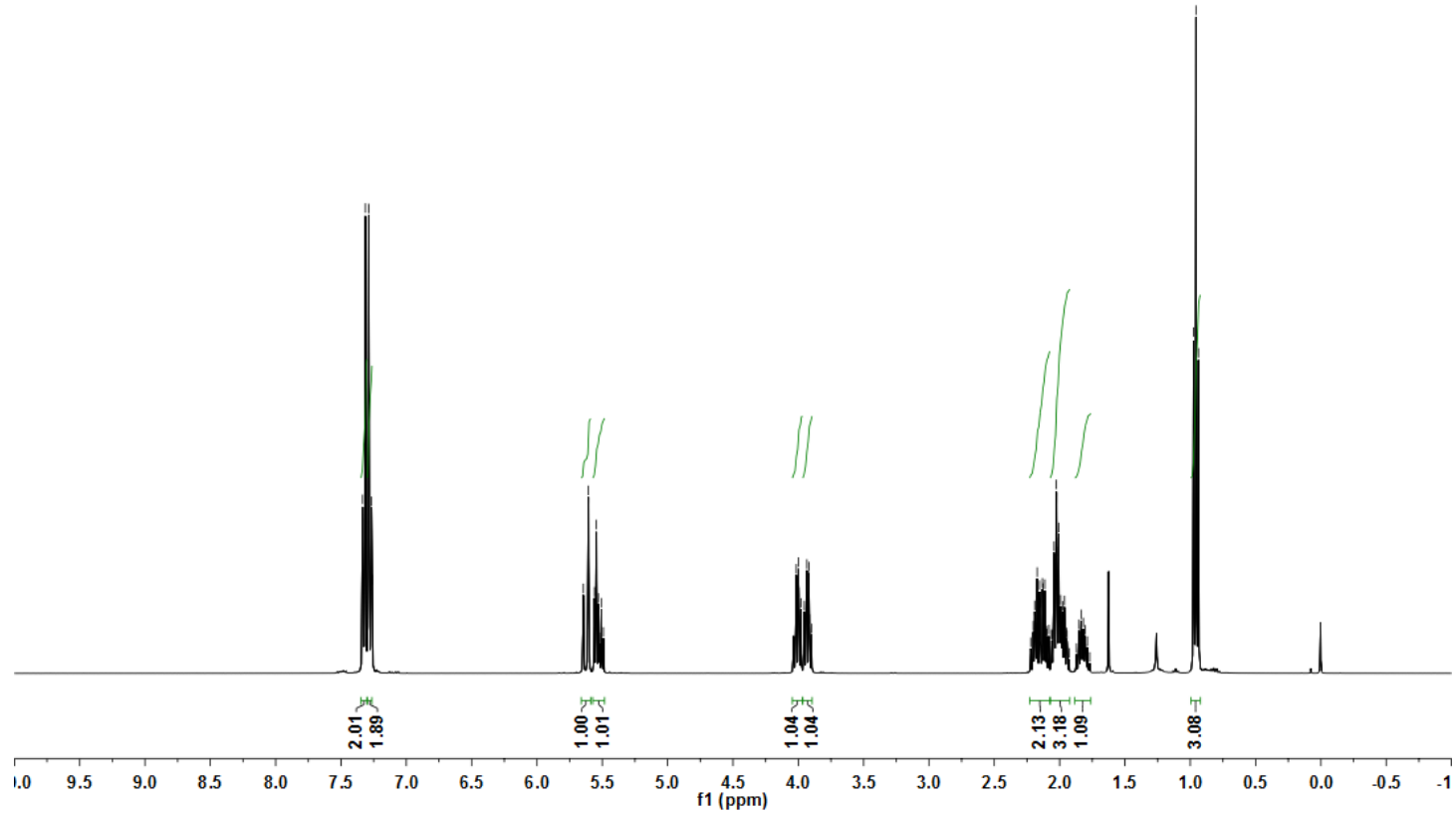

$\mathbb{1}_{01}^{0}$

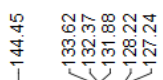

음욜

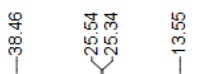

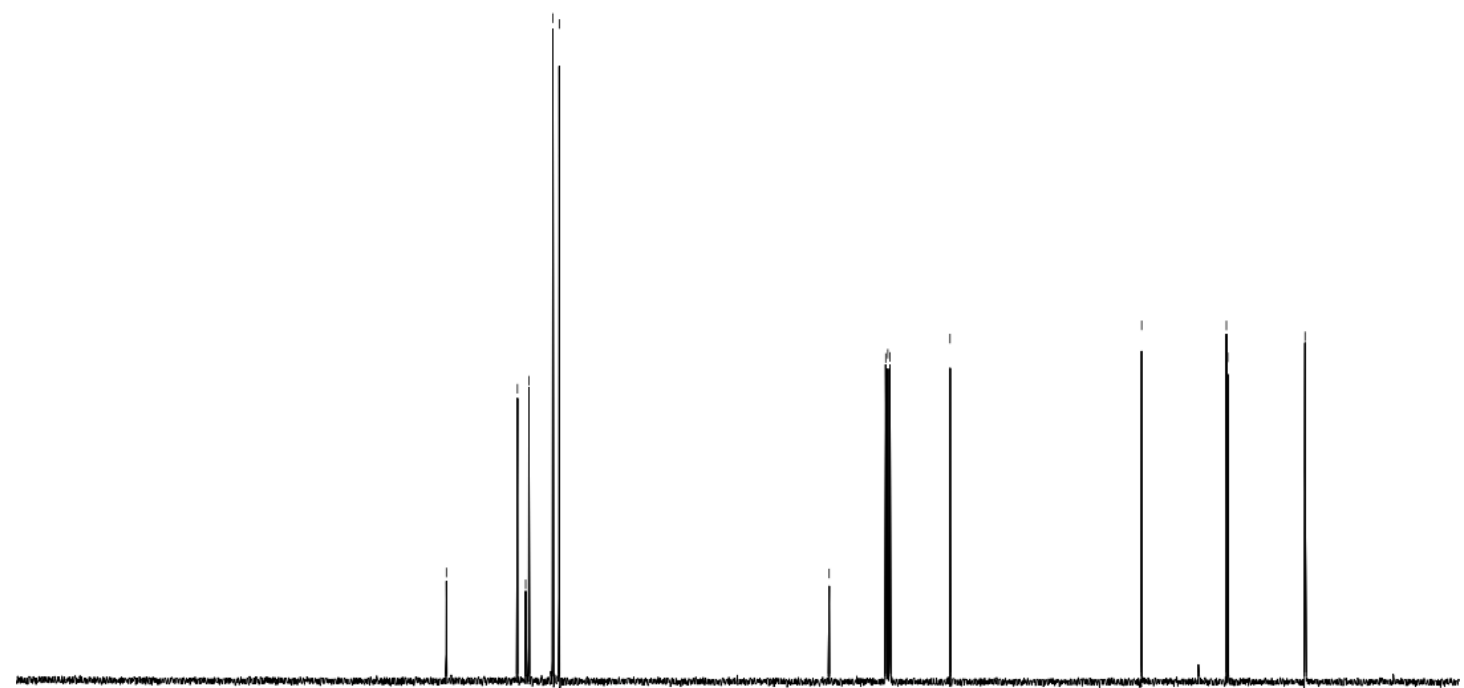

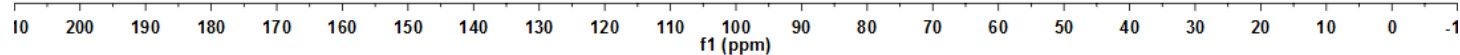


${ }^{1} \mathrm{H}$ NMR (400 MHz, $\left.\mathrm{CDCl}_{3}\right)$ and ${ }^{13} \mathrm{C}$ NMR (101 $\left.\mathrm{MHz}, \mathrm{CDCl}_{3}\right)$ of $2 \mathrm{~m}$

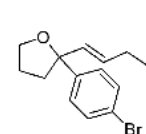

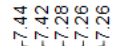

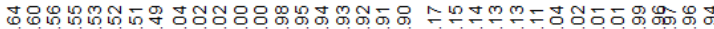

证

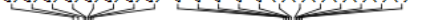
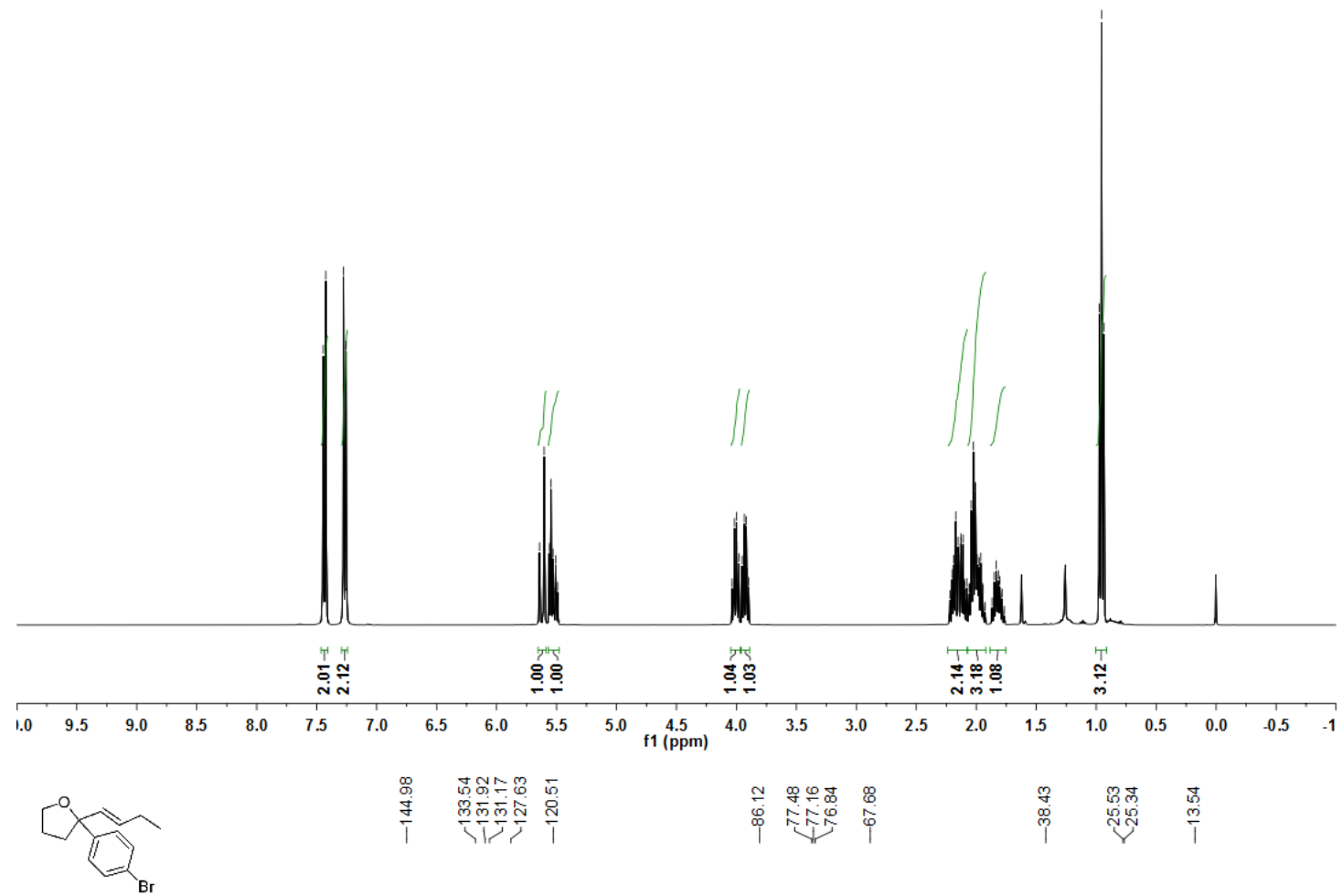

广

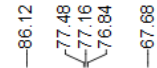

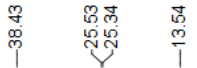

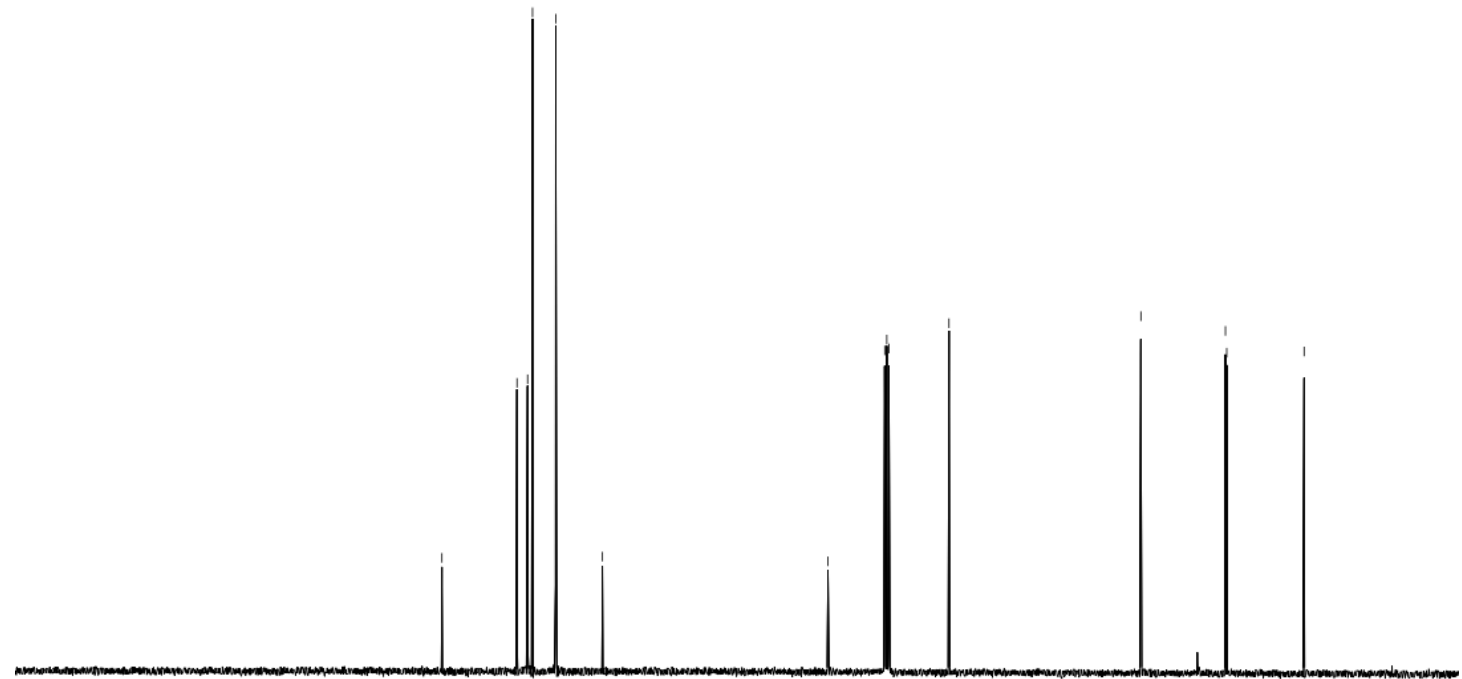

\begin{tabular}{|llllllllllllllllllllllll}
\hline 10 & 200 & 190 & 180 & 170 & 160 & 150 & 140 & 130 & 120 & 110 & 100 & 90 & 80 & 70 & 60 & 50 & 40 & 30 & 20 & 10 & 0 & -1
\end{tabular} 
${ }^{1} \mathrm{H}$ NMR (400 MHz, $\mathrm{CDCl}_{3}$ ) and ${ }^{13} \mathrm{C} \mathrm{NMR} \mathrm{(101} \mathrm{MHz,} \mathrm{CDCl}_{3}$ ) of $2 \mathrm{n}$

这

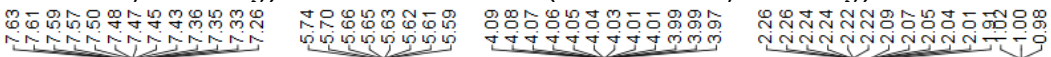
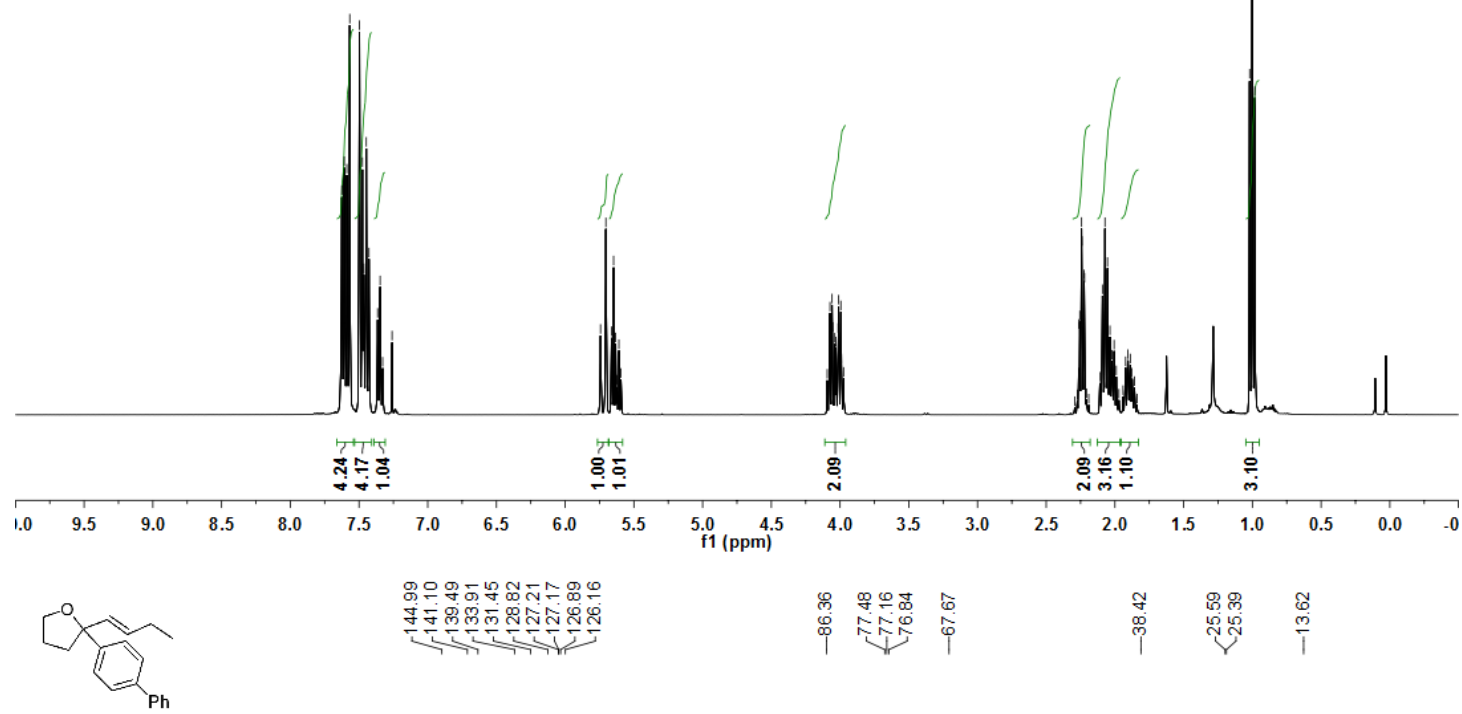

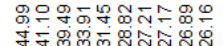

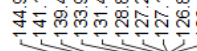

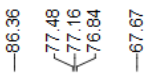

每

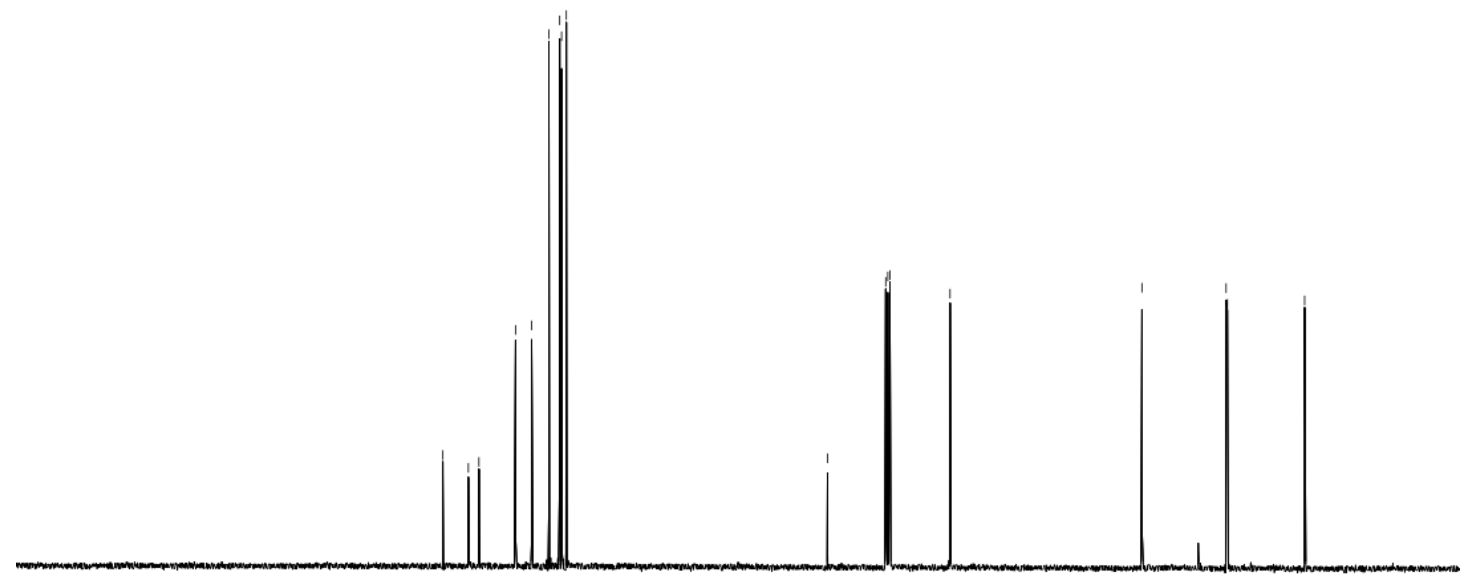

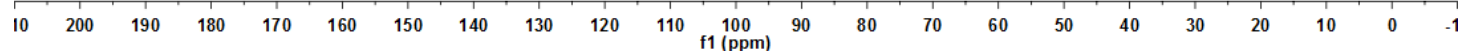


${ }^{1} \mathrm{H}$ NMR (400 MHz, $\left.\mathrm{CDCl}_{3}\right)$ and ${ }^{13} \mathrm{C}$ NMR (101 $\left.\mathrm{MHz}, \mathrm{CDCl}_{3}\right)$ of 20<smiles>C1=CC2=C[Ge][Ge]2=C1</smiles>

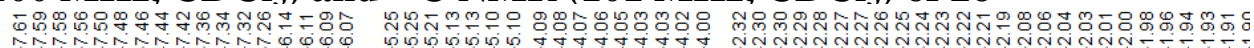
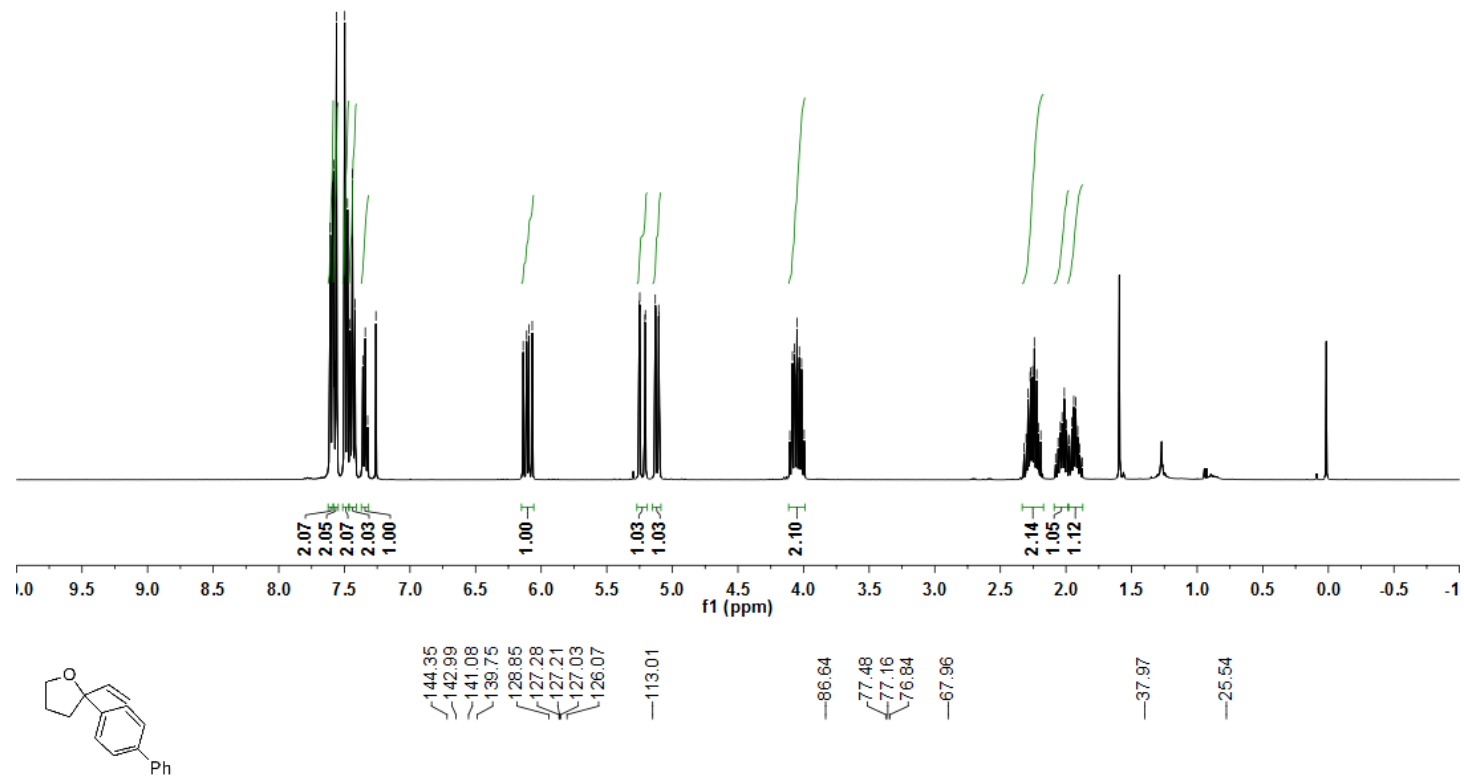

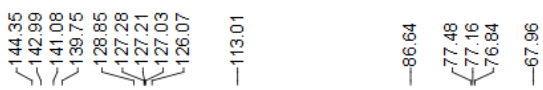

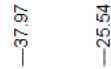

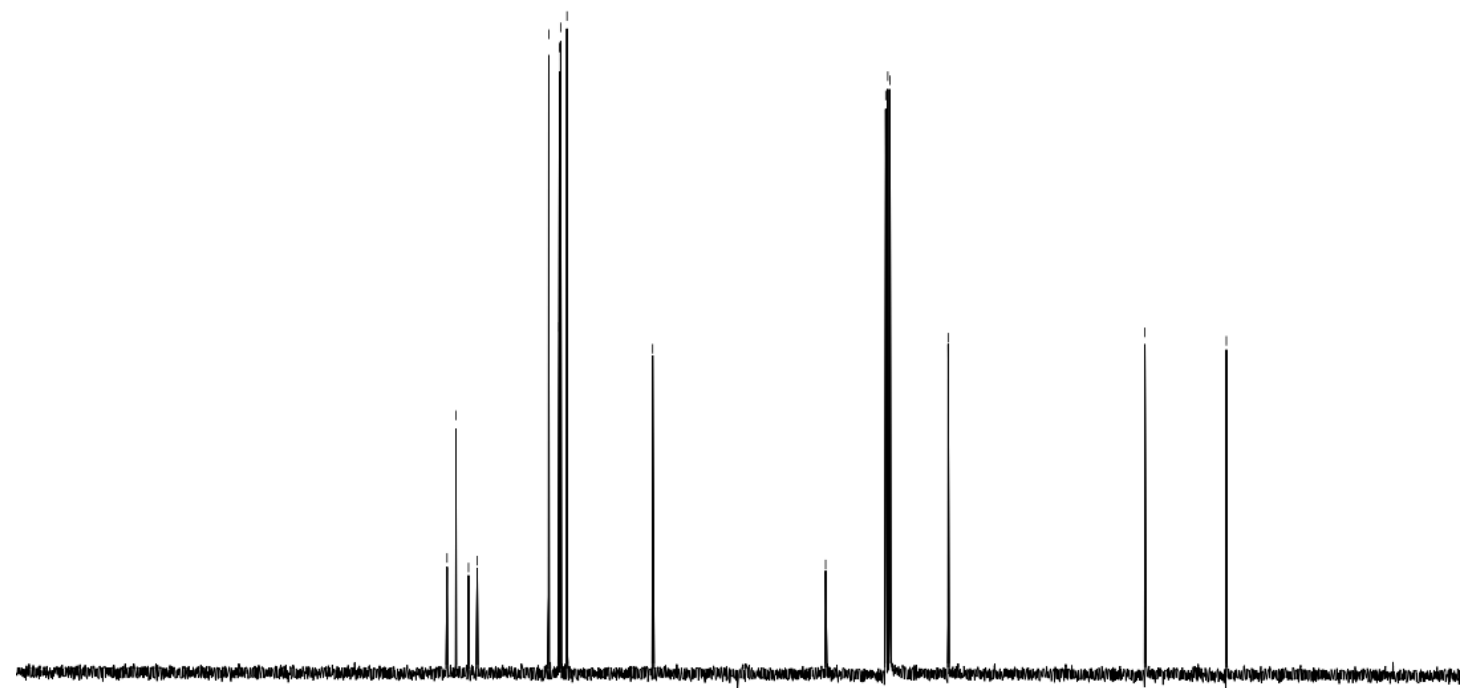

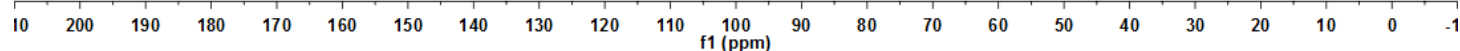


${ }^{1} \mathrm{H}$ NMR (400 MHz, $\left.\mathrm{CDCl}_{3}\right)$ and ${ }^{13} \mathrm{C}$ NMR (101 $\left.\mathrm{MHz}, \mathrm{CDCl}_{3}\right)$ of 2p

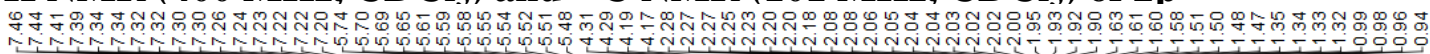
You

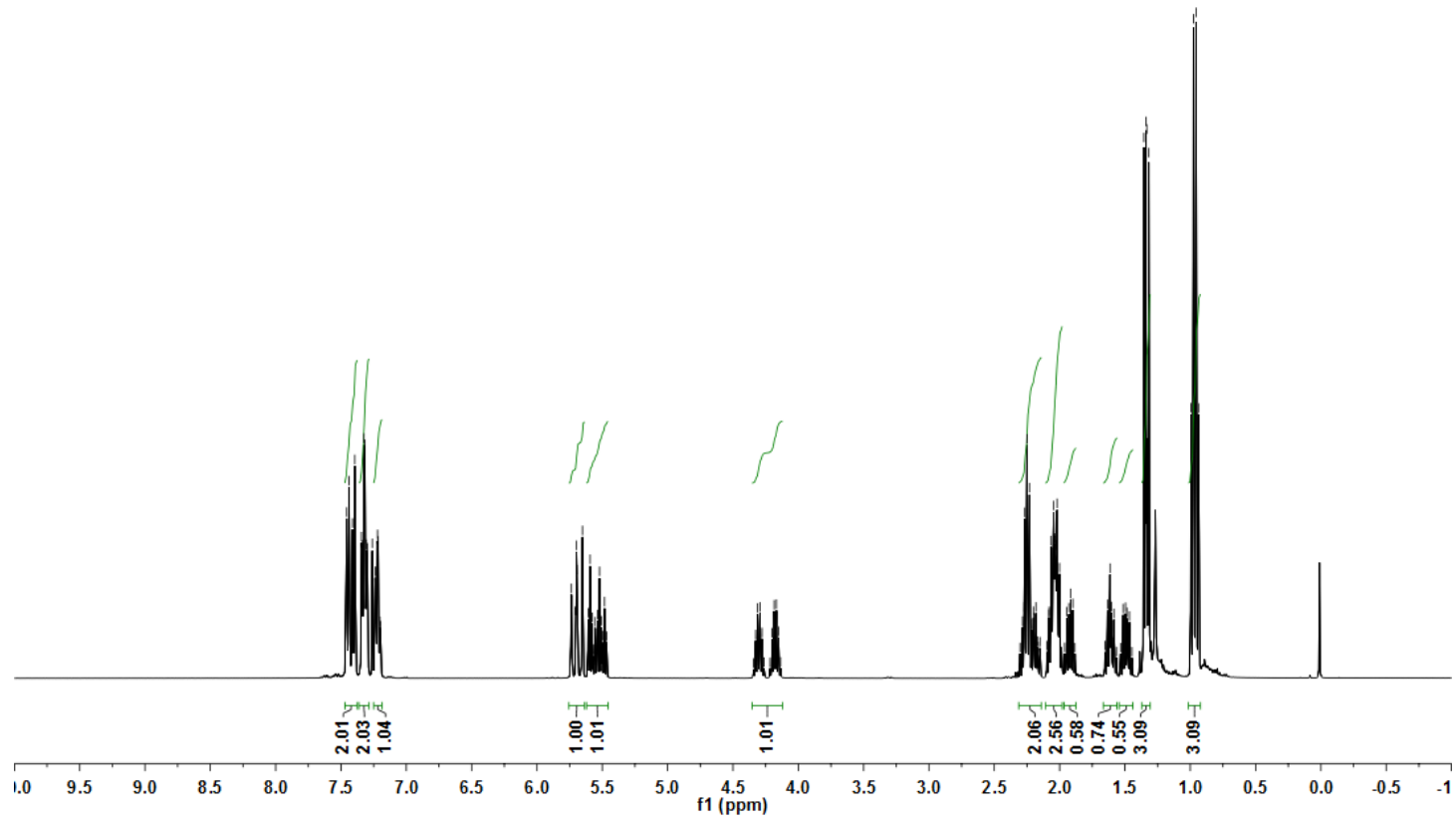

U

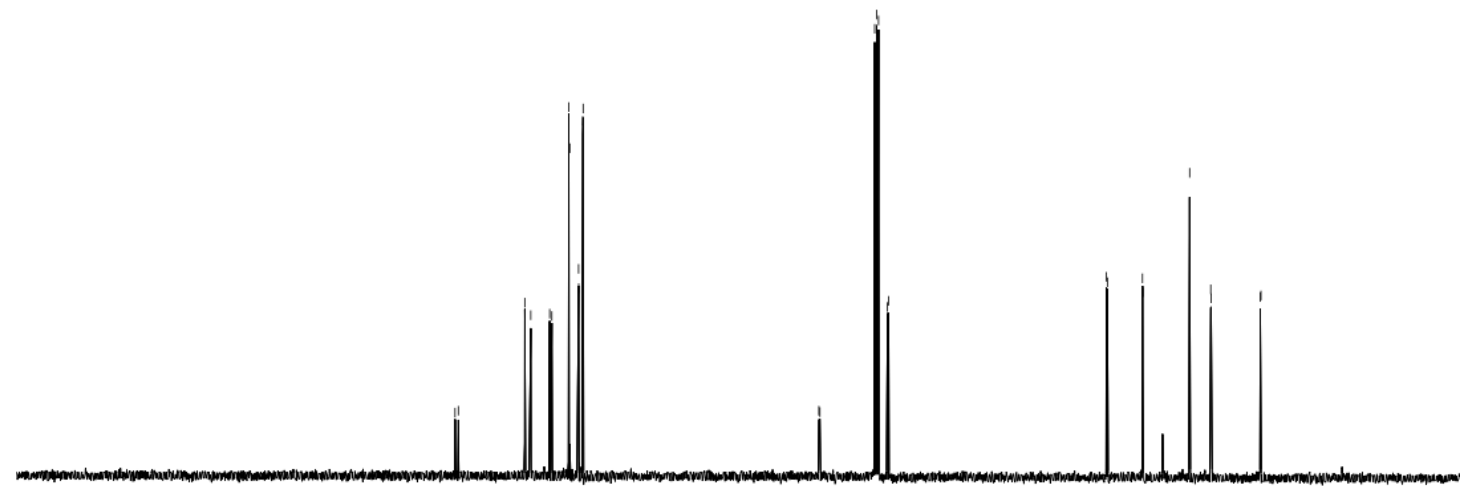

$\begin{array}{lllllllllllllllllllllll}210 & 200 & 190 & 180 & 170 & 160 & 150 & 140 & 130 & 120 & 110 & 100 & 90 & 80 & 70 & 60 & 50 & 40 & 30 & 20 & 10 & 0 & -10\end{array}$ 
${ }^{1} \mathrm{H}$ NMR (400 MHz, $\left.\mathrm{CDCl}_{3}\right)$ and ${ }^{13} \mathrm{C}$ NMR (101 $\left.\mathrm{MHz}, \mathrm{CDCl}_{3}\right)$ of $2 \mathrm{q}$
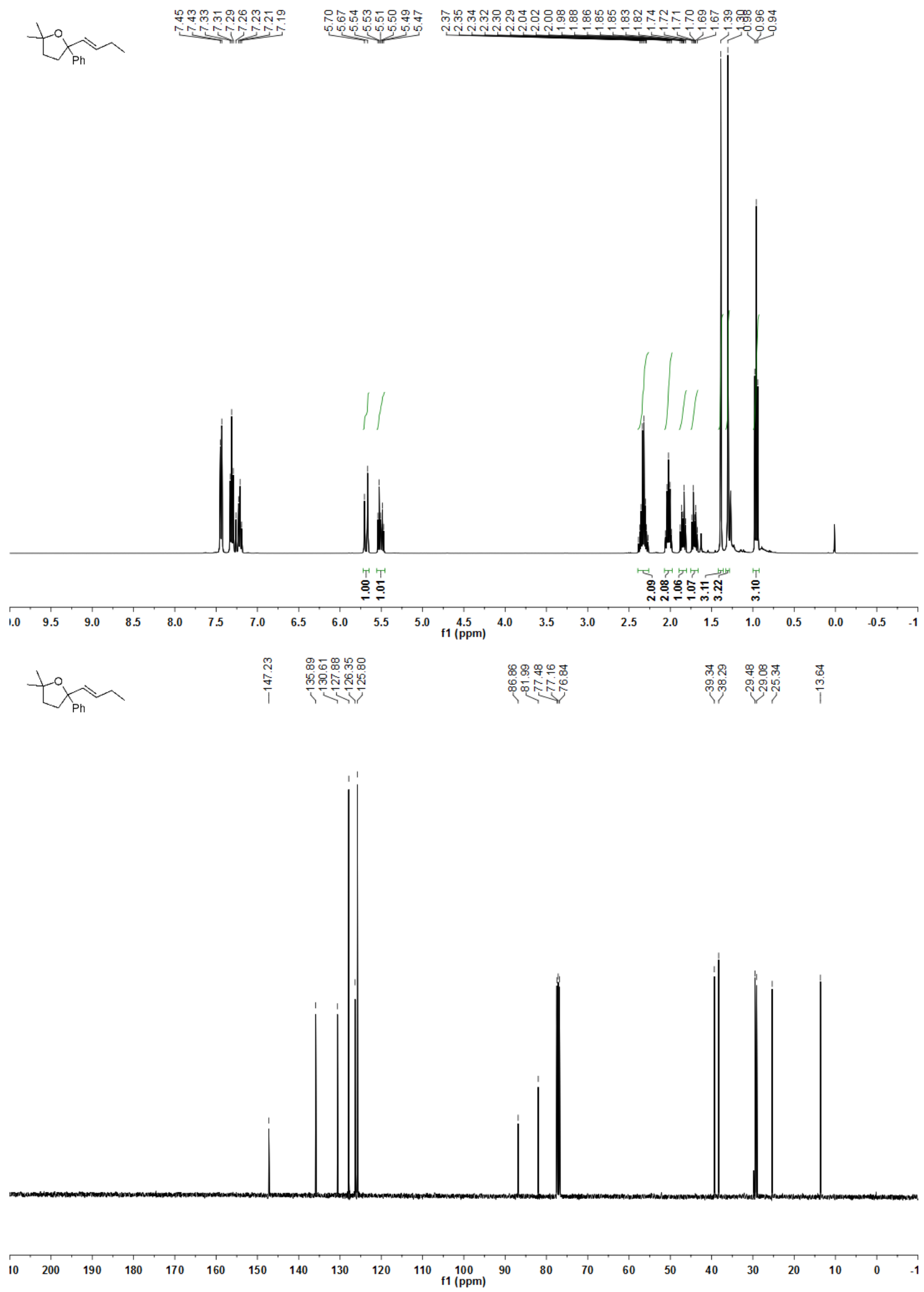
${ }^{1} \mathrm{H}$ NMR (400 MHz, $\left.\mathrm{CDCl}_{3}\right)$ and ${ }^{13} \mathrm{C}$ NMR (101 MHz, $\left.\mathrm{CDCl}_{3}\right)$ of 2r

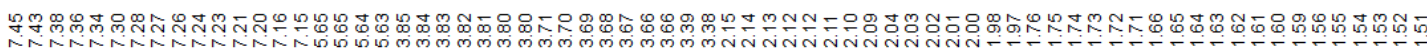
$\int_{\mathrm{Ph}}^{\mathrm{Ph}}$

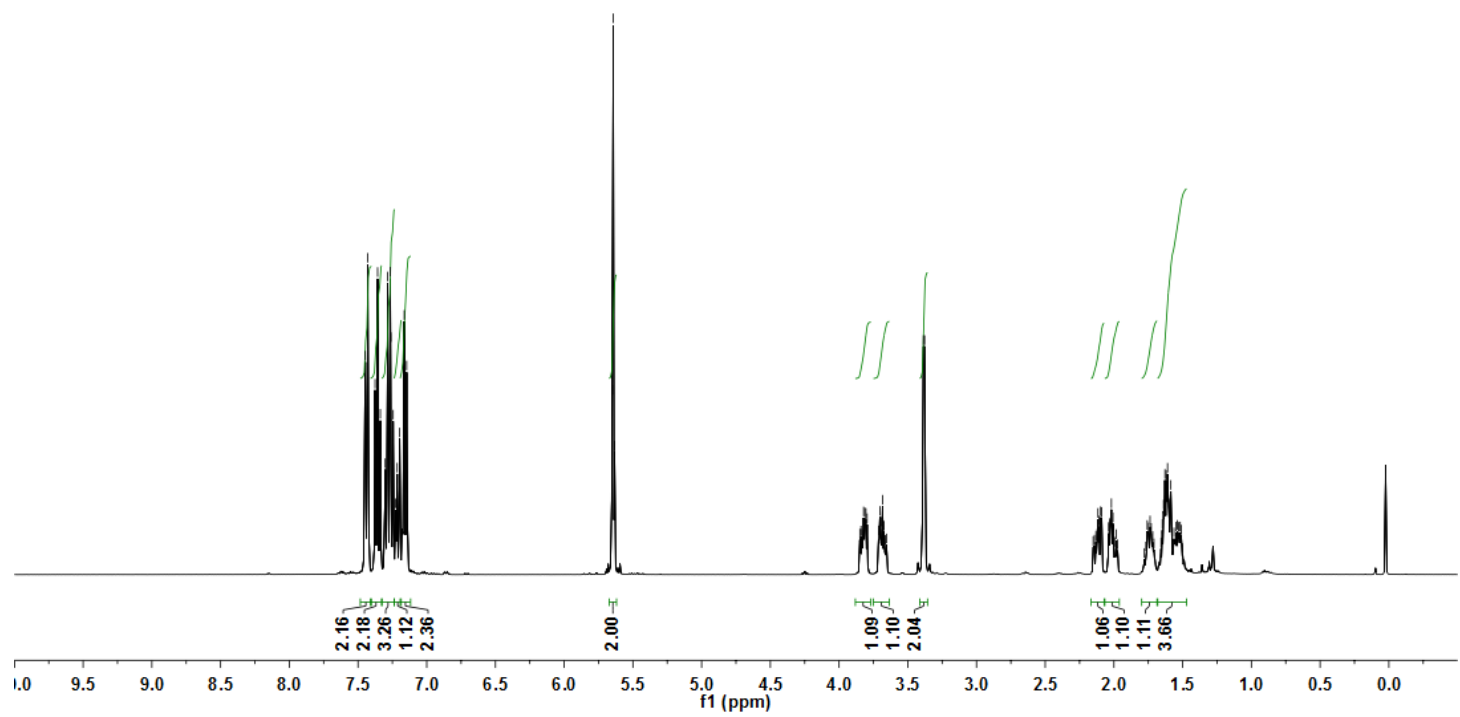

[

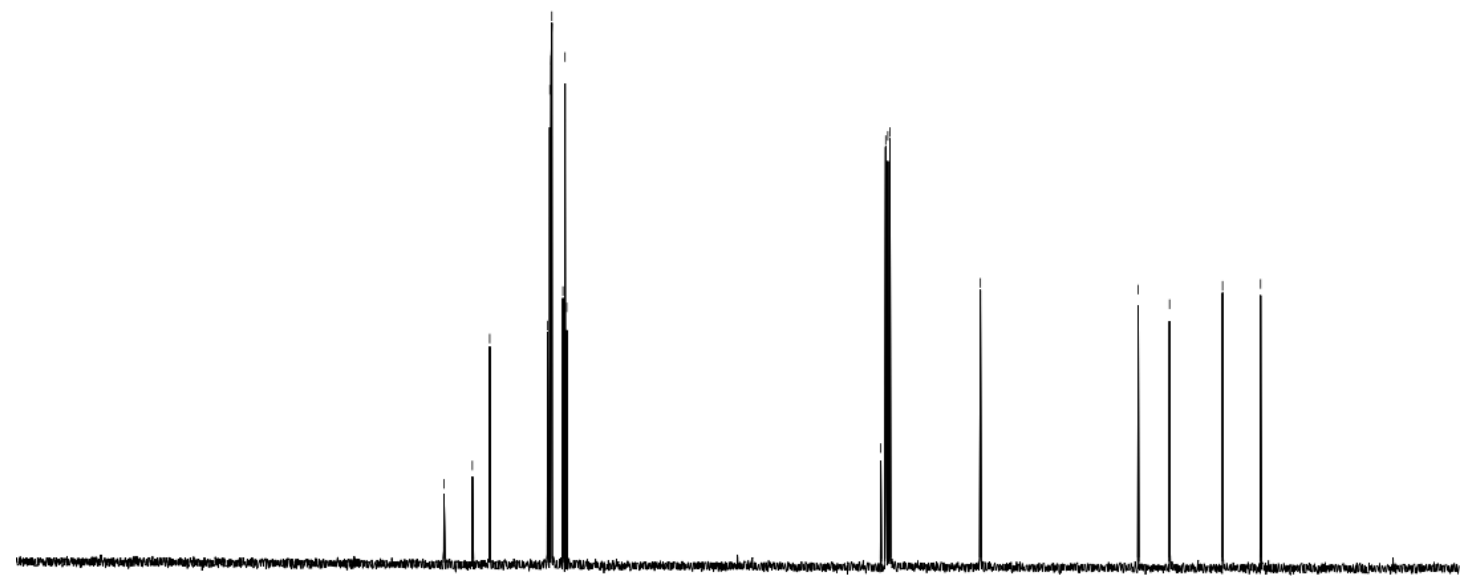

$\begin{array}{lllllllllllllllllllllllllllllllll}10 & 200 & 190 & 180 & 170 & 160 & 150 & 140 & 130 & 120 & 110 & \begin{array}{c}100 \\ \mathrm{f} 1(\mathrm{ppm})\end{array} & 90 & 80 & 70 & 60 & 50 & 40 & 30 & 20 & 10 & 0 & -1\end{array}$ 
${ }^{1} \mathrm{H}$ NMR (400 MHz, $\left.\mathrm{CDCl}_{3}\right)$ and ${ }^{13} \mathrm{C}$ NMR (101 MHz, $\left.\mathrm{CDCl}_{3}\right)$ of $2 \mathrm{~s}$
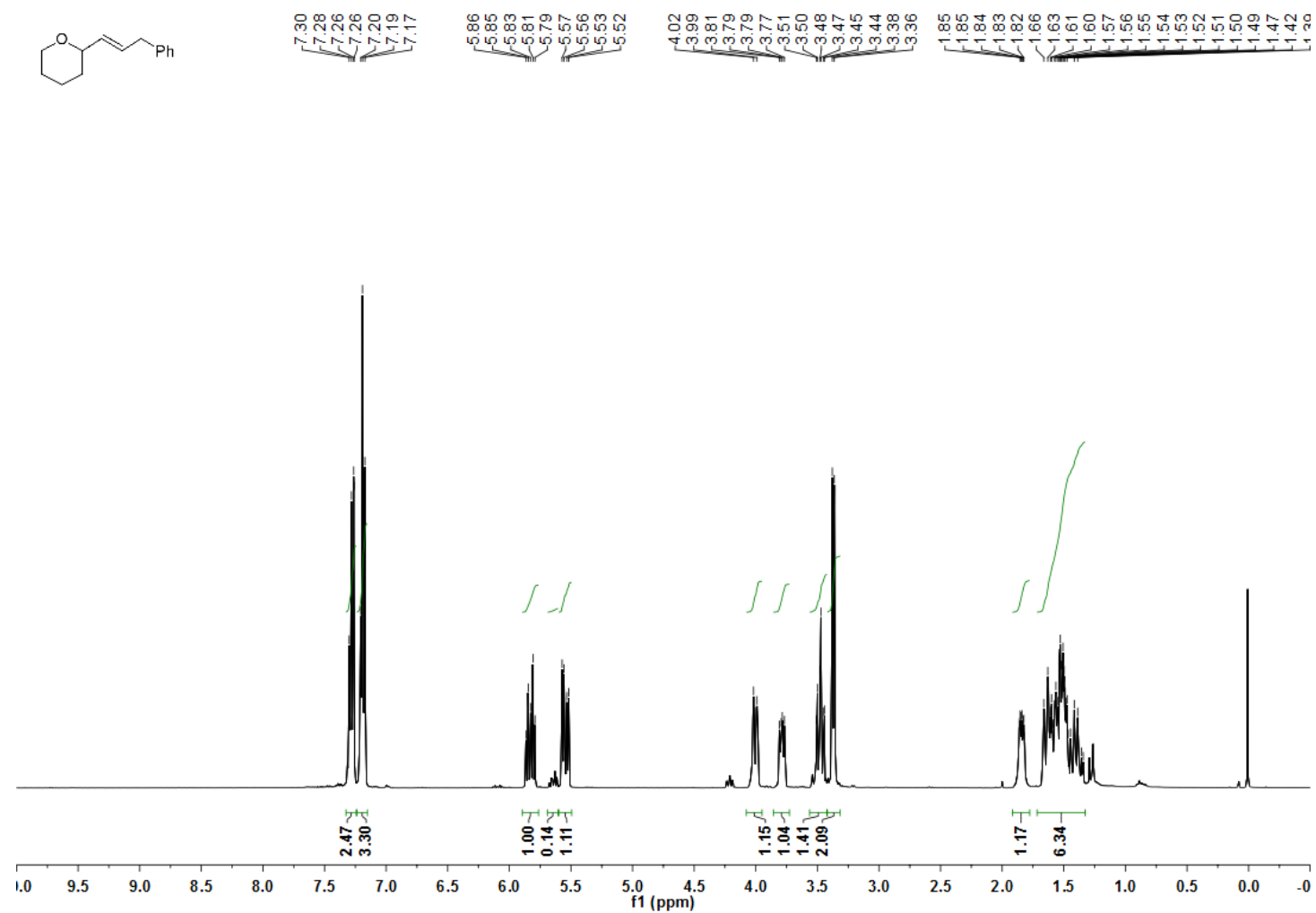

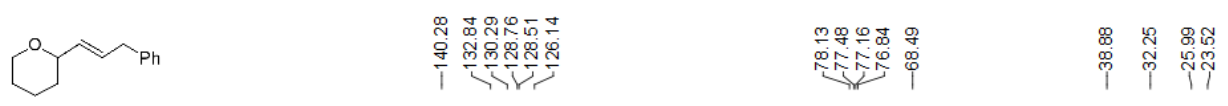

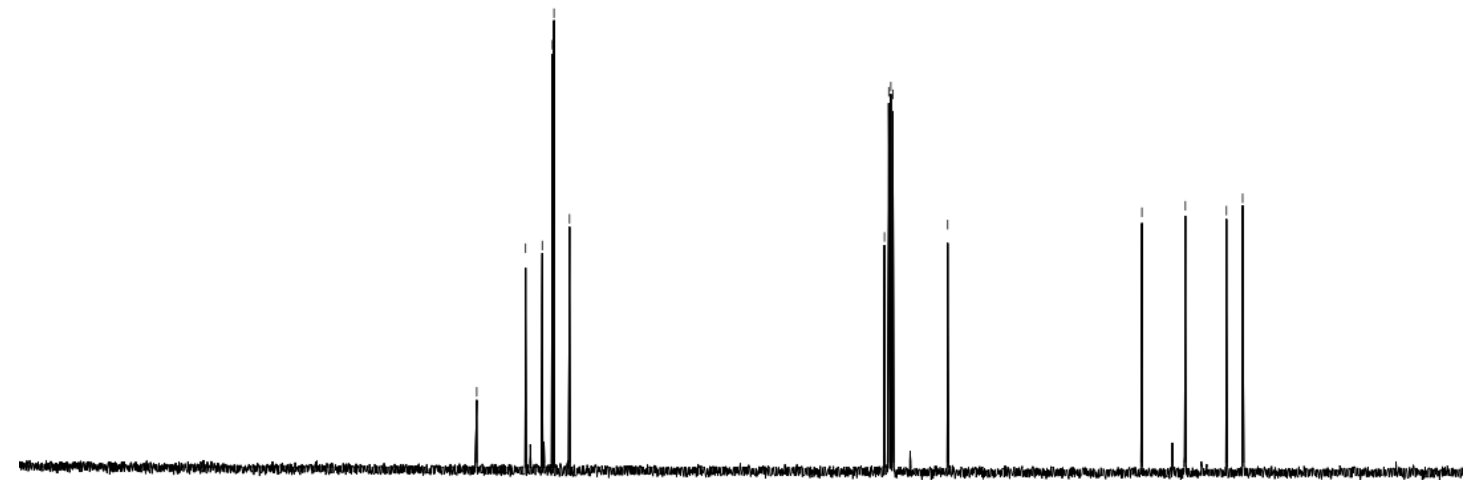

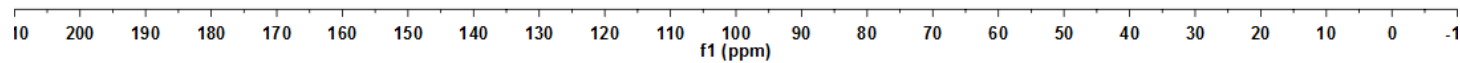


${ }^{1} \mathrm{H}$ NMR (400 MHz, $\left.\mathrm{CDCl}_{3}\right)$ and ${ }^{13} \mathrm{C}$ NMR $\left(101 \mathrm{MHz}, \mathrm{CDCl}_{3}\right)$ of $5 \mathrm{a}$
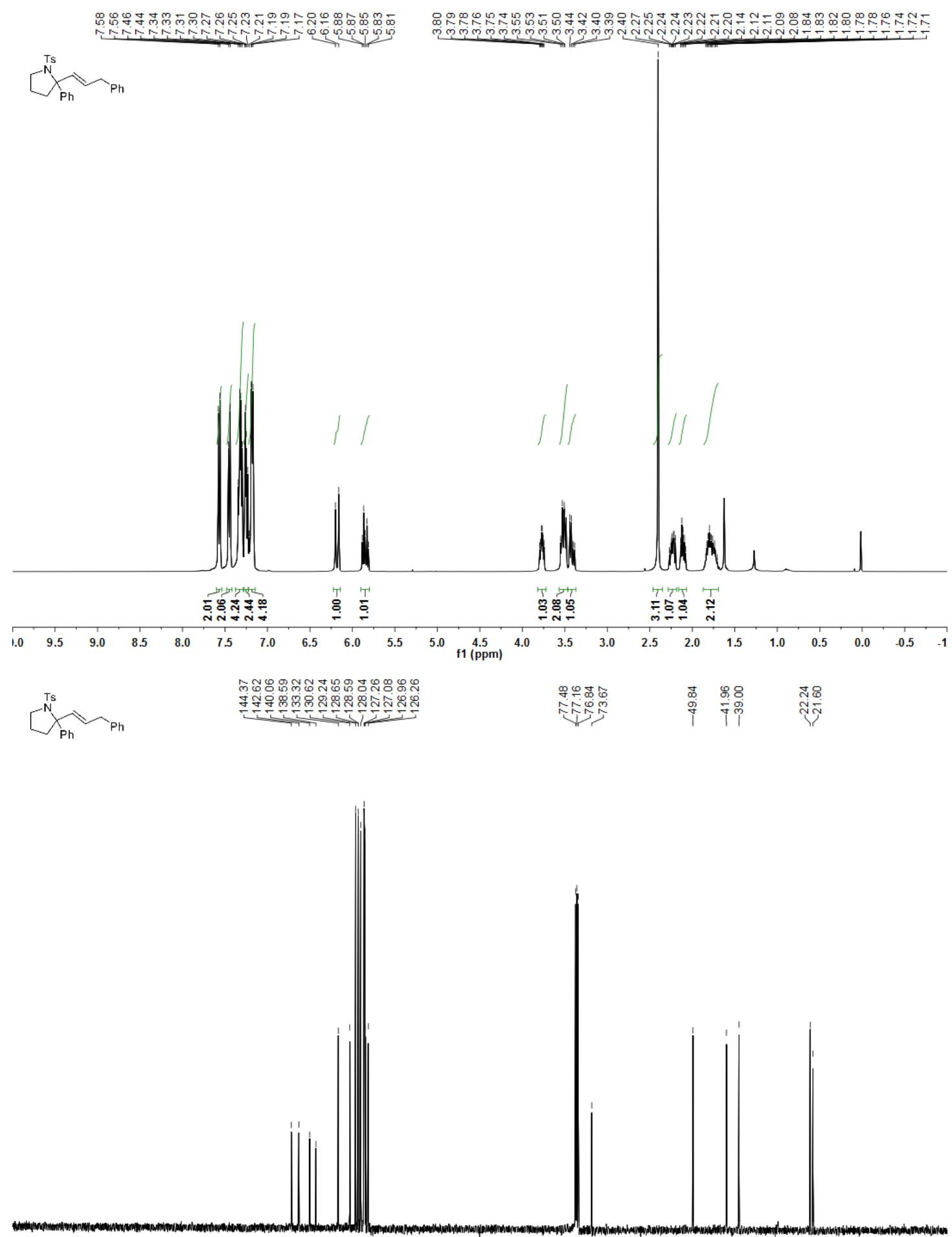

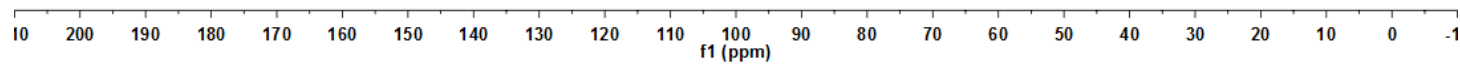


${ }^{1} \mathrm{H}$ NMR (400 MHz, $\left.\mathrm{CDCl}_{3}\right)$ and ${ }^{13} \mathrm{C}$ NMR (101 MHz, $\left.\mathrm{CDCl}_{3}\right)$ of $5 \mathrm{~b}$ $D_{\text {Me }}^{\text {Ts }}$

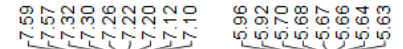

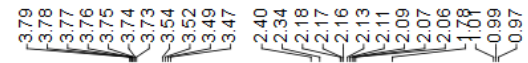
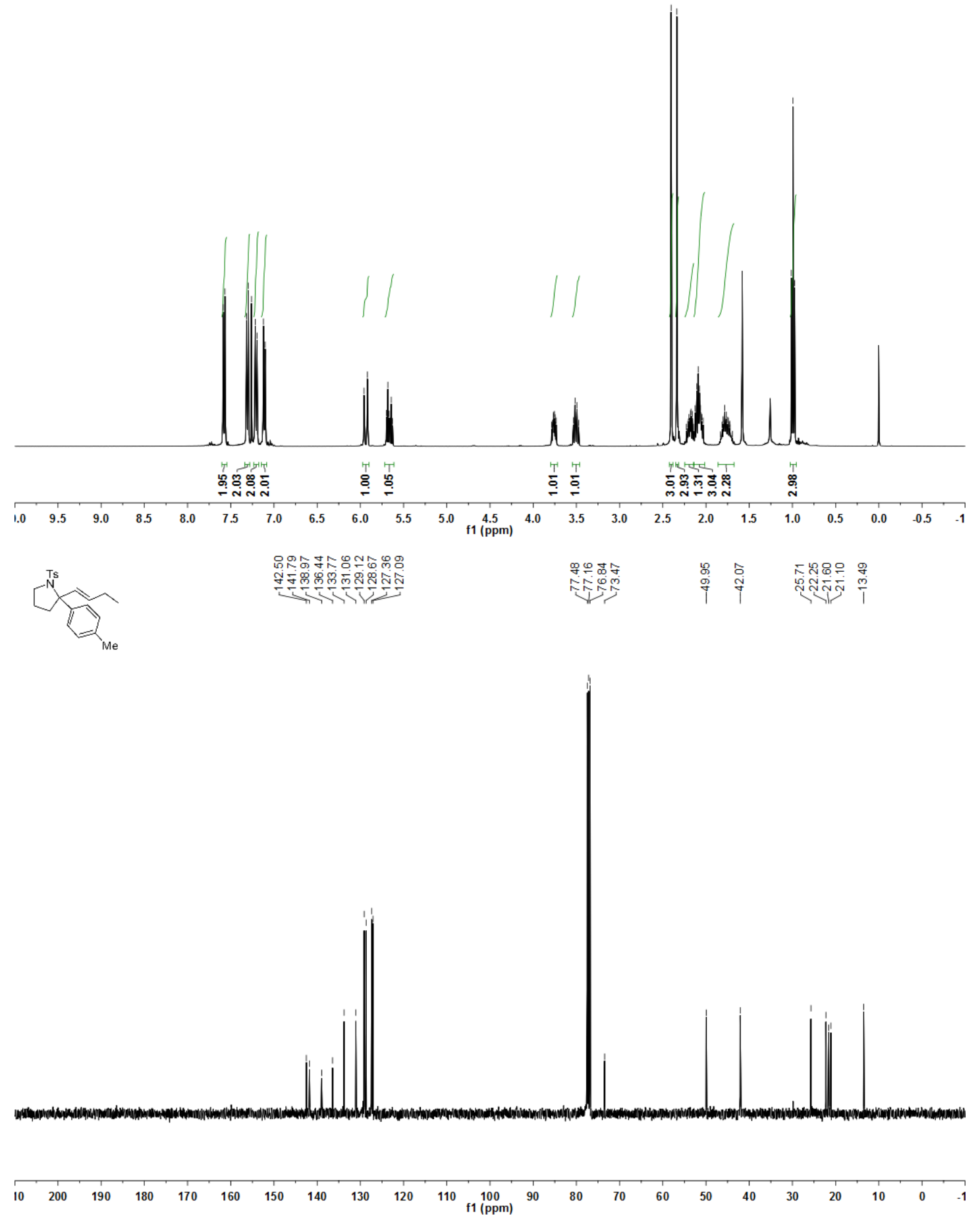
${ }^{1} \mathrm{H}$ NMR (400 MHz, $\left.\mathrm{CDCl}_{3}\right)$ and $\left.{ }^{13} \mathrm{C} \mathrm{NMR} \mathrm{(101} \mathrm{MHz,} \mathrm{CDCl}_{3}\right)$ of $5 \mathrm{c}$<smiles>[Te]=C1CCCC1</smiles>
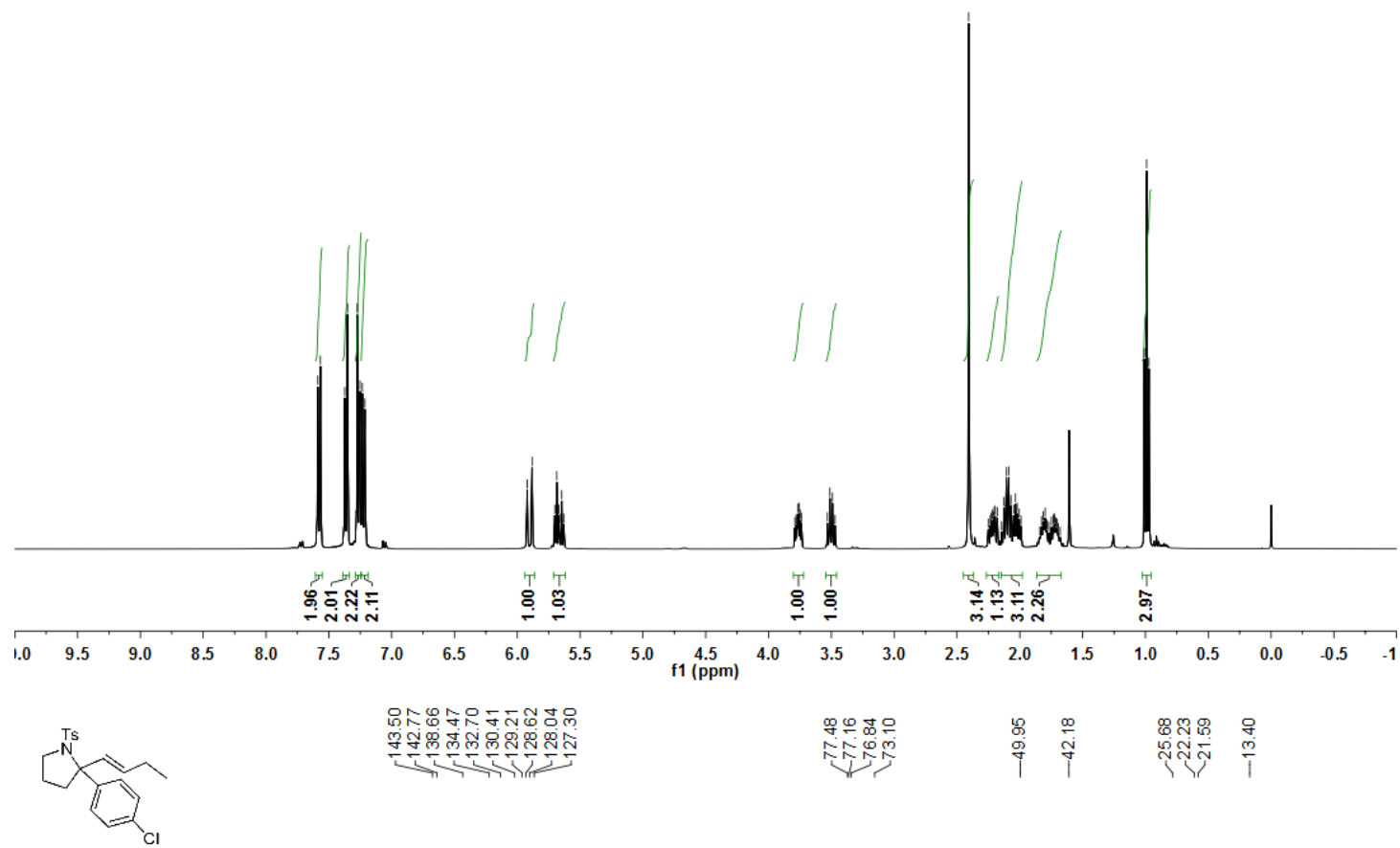

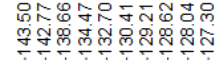

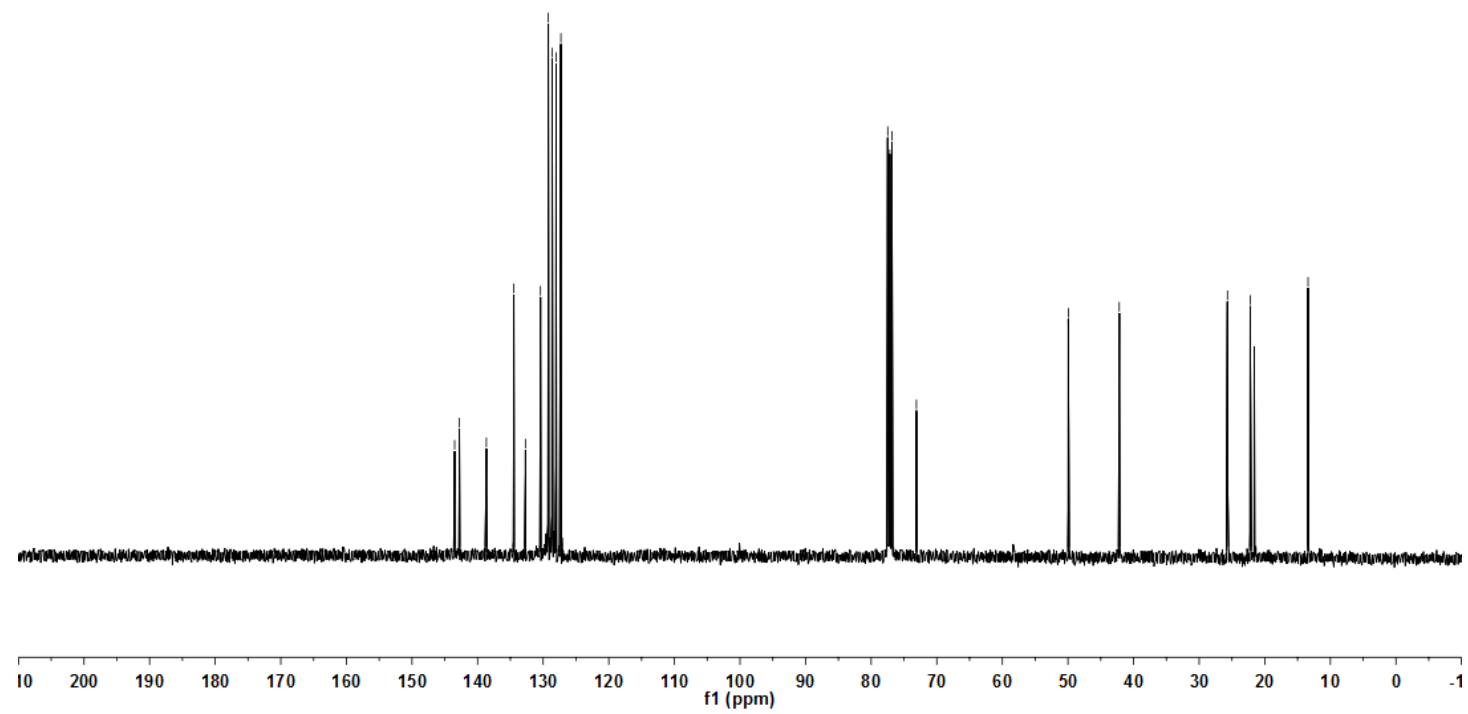


${ }^{1} \mathrm{H}$ NMR (400 MHz, $\left.\mathrm{CDCl}_{3}\right)$ and ${ }^{13} \mathrm{C}$ NMR (101 MHz, $\left.\mathrm{CDCl}_{3}\right)$ of $5 \mathrm{~d}$
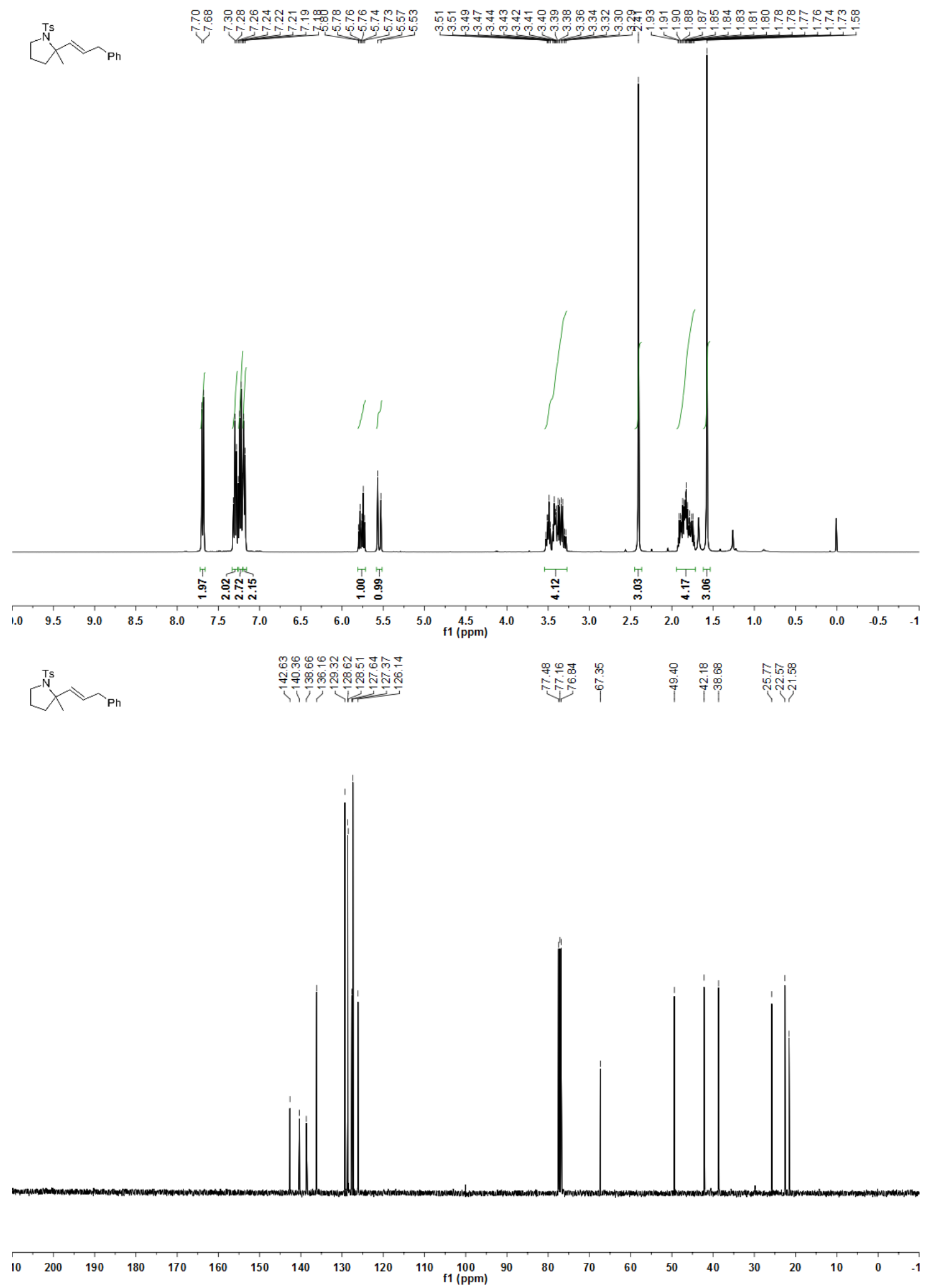
${ }^{1} \mathrm{H}$ NMR (400 MHz, $\left.\mathrm{CDCl}_{3}\right)$ and ${ }^{13} \mathrm{C}$ NMR (101 MHz, $\left.\mathrm{CDCl}_{3}\right)$ of $5 \mathrm{e}$
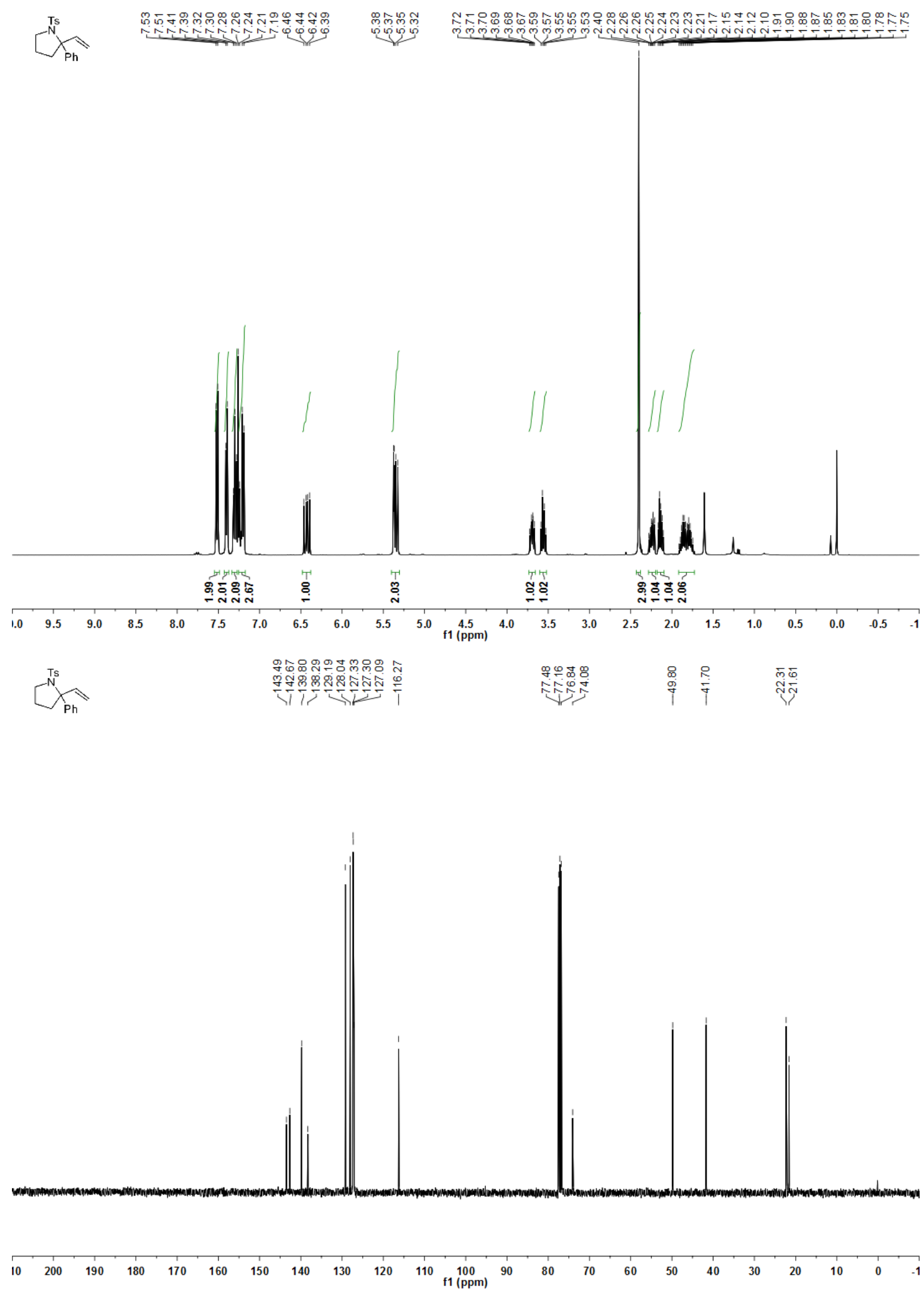
${ }^{1} \mathrm{H}$ NMR (400 MHz, $\left.\mathrm{CDCl}_{3}\right)$ and ${ }^{13} \mathrm{C}$ NMR (101 MHz, $\left.\mathrm{CDCl}_{3}\right)$ of $5 f$ 而认出

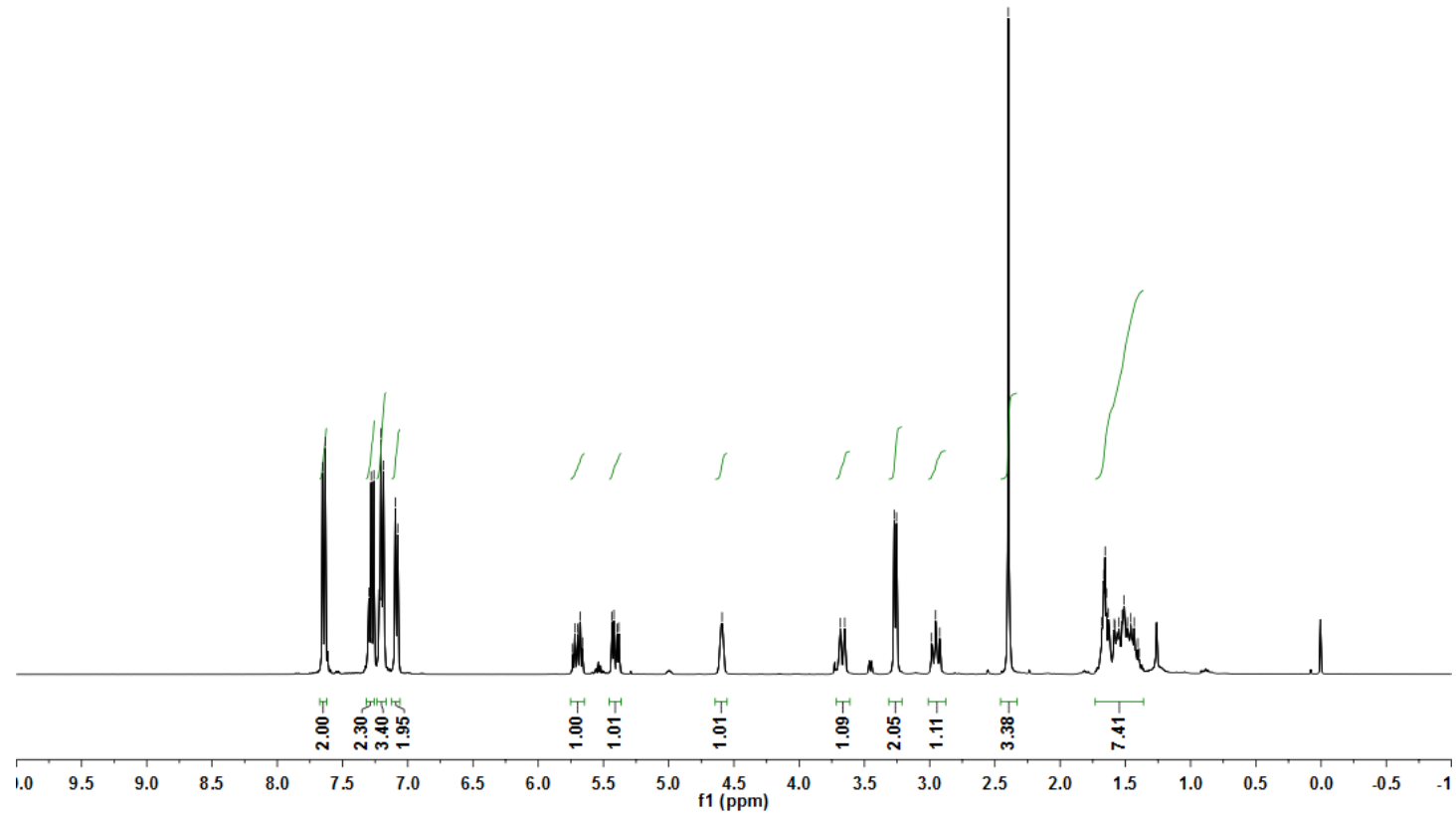
ר

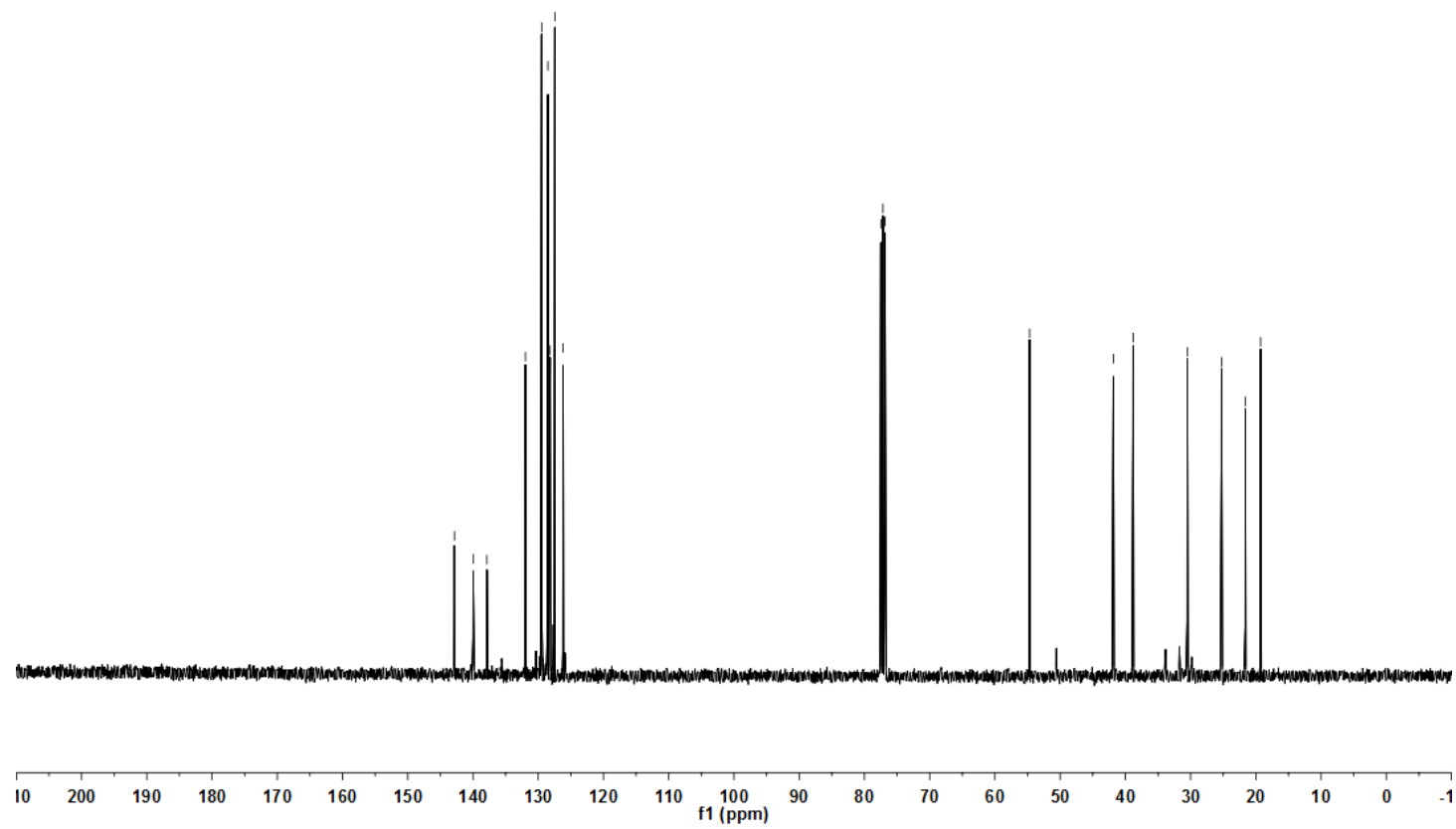


${ }^{1} \mathrm{H}$ NMR $\left(400 \mathrm{MHz}, \mathrm{CDCl}_{3}\right)$ and ${ }^{13} \mathrm{C}$ NMR $\left(101 \mathrm{MHz}, \mathrm{CDCl}_{3}\right)$ of $6 \mathrm{a}$

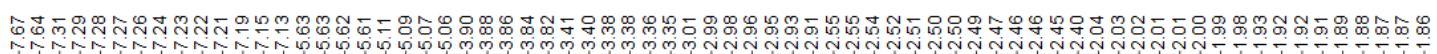
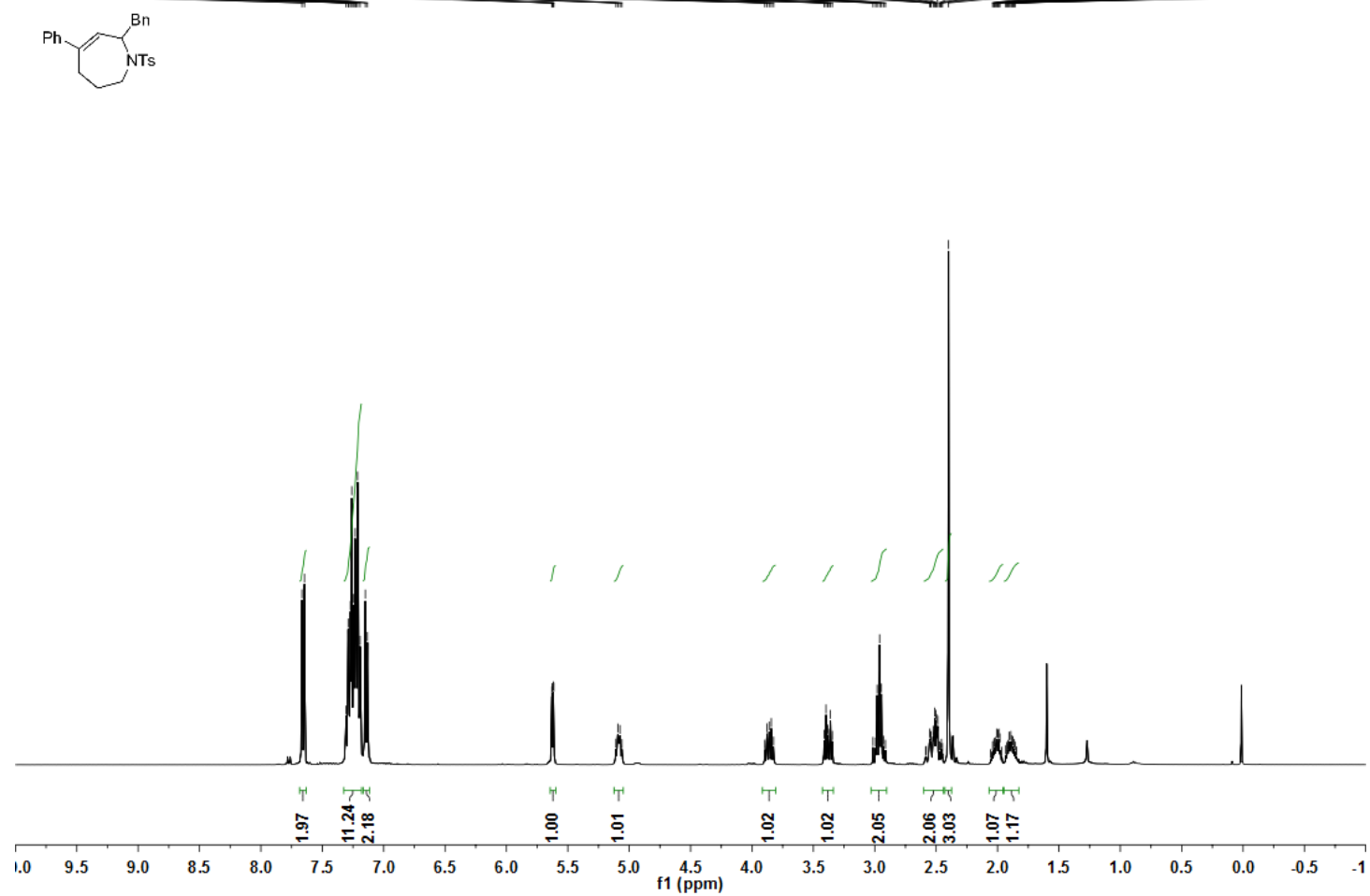

L

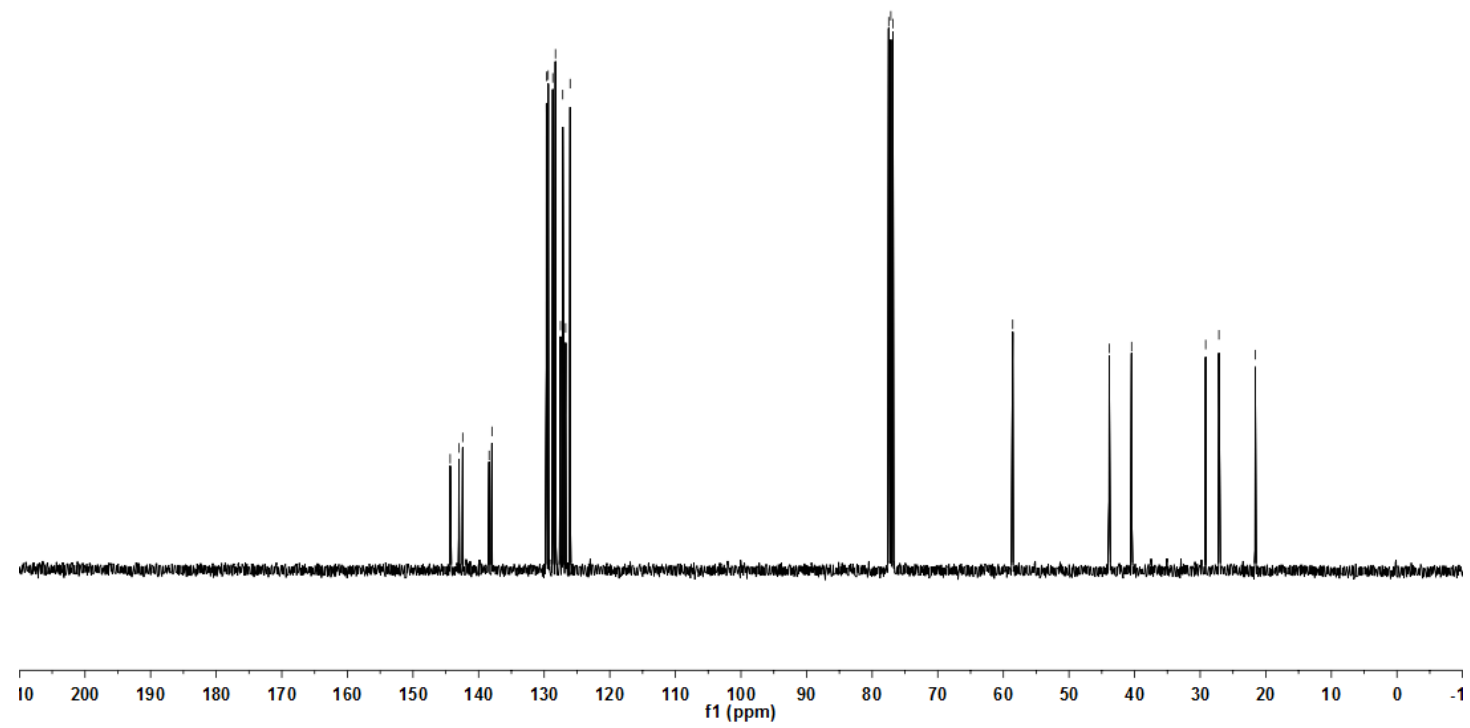


${ }^{1} \mathrm{H}$ NMR (400 MHz, $\left.\mathrm{CDCl}_{3}\right)$ and ${ }^{13} \mathrm{C}$ NMR $\left(101 \mathrm{MHz}, \mathrm{CDCl}_{3}\right)$ of $6 \mathrm{~b}$
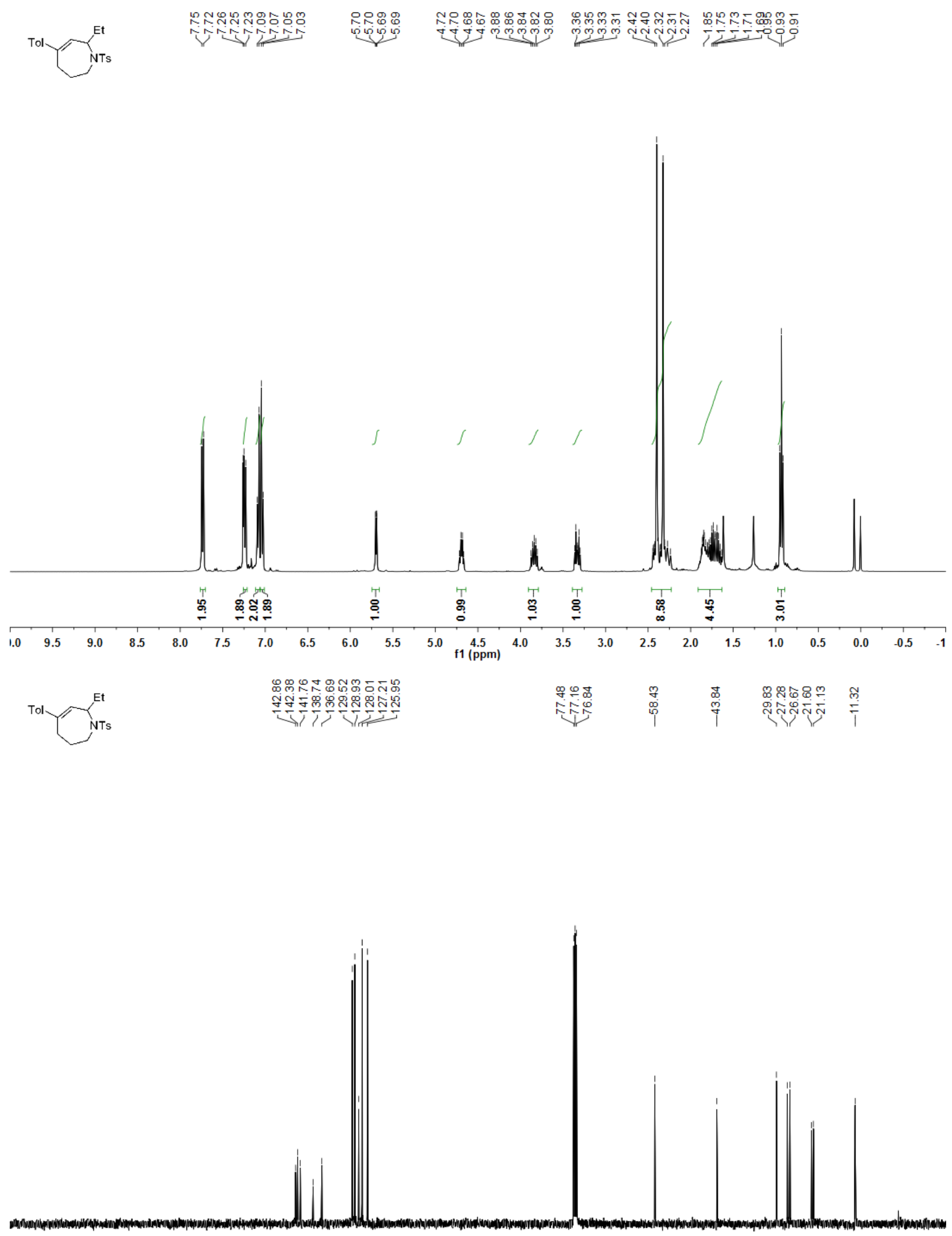

\begin{tabular}{lllllllllllllllllllllll}
\hline 10 & 200 & 190 & 180 & 170 & 160 & 150 & 140 & 130 & 120 & 110 & $\begin{array}{c}100 \\
\mathrm{f} 1(\mathrm{ppm})\end{array}$ & 90 & 80 & 70 & 60 & 50 & 40 & 30 & 20 & 10 & 0 & -1
\end{tabular} 
${ }^{1} \mathrm{H}$ NMR (400 MHz, $\left.\mathrm{CDCl}_{3}\right)$ and ${ }^{13} \mathrm{C}$ NMR (101 MHz, $\left.\mathrm{CDCl}_{3}\right)$ of $6 \mathrm{c}$

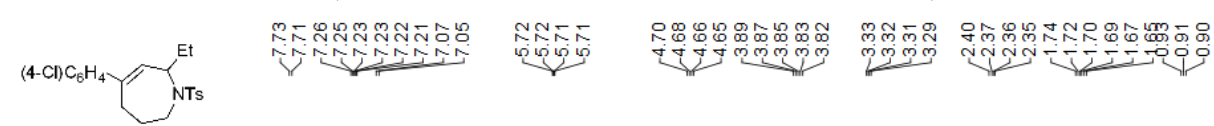

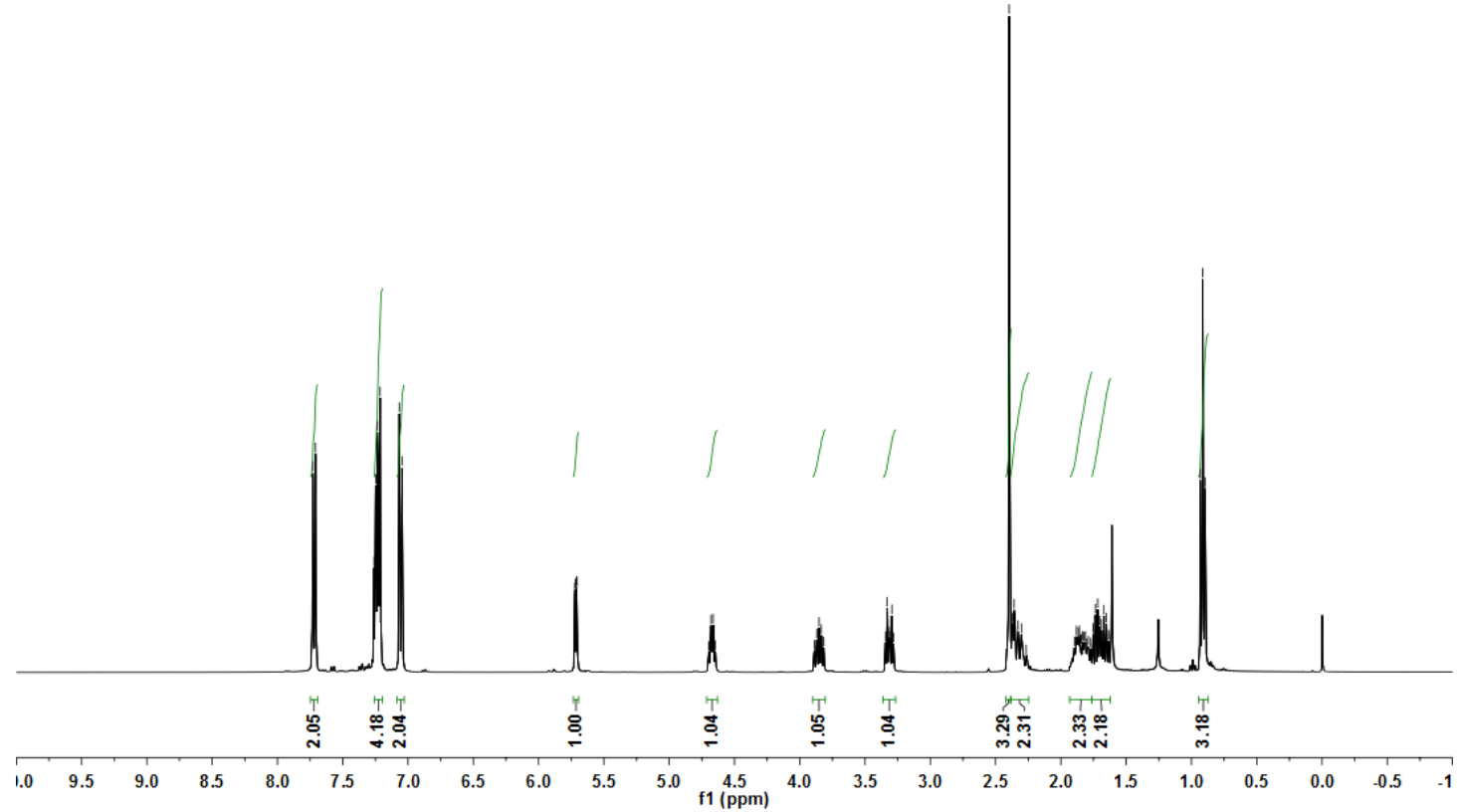

(4-CI) $\mathrm{C}_{6} \mathrm{H}_{4} Y_{\mathrm{NTS}}^{\mathrm{Et}}$

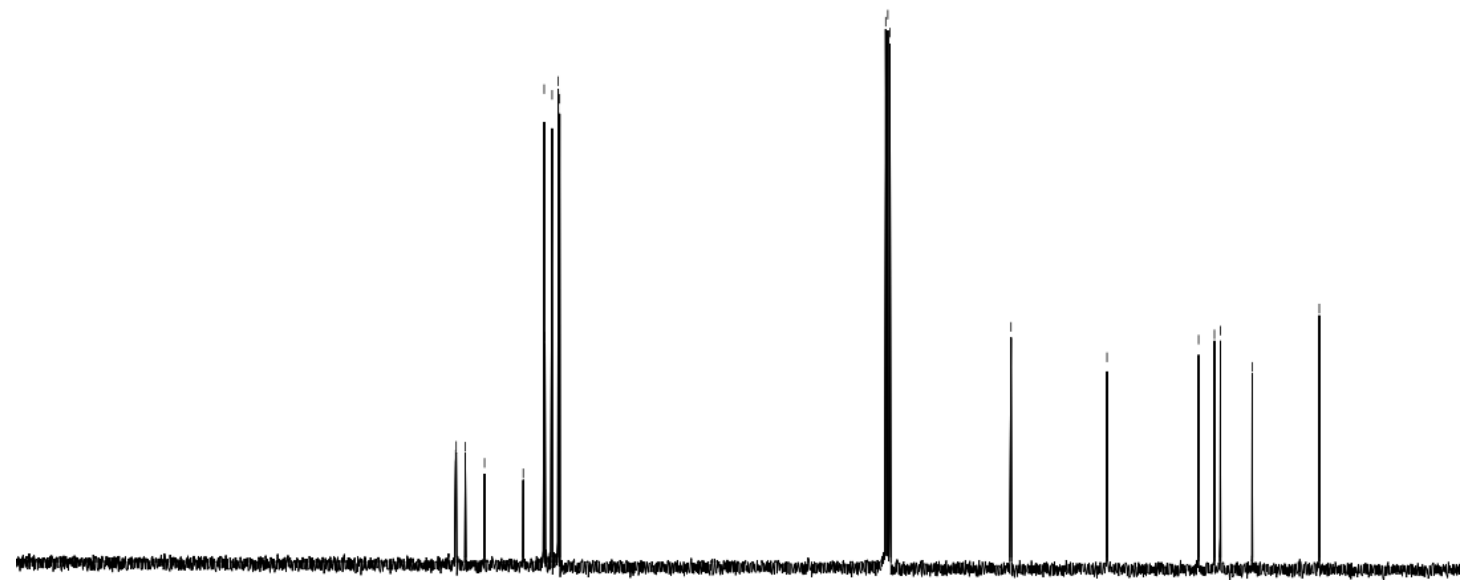

\begin{tabular}{|lllllllllllllllllllllllll}
\hline 10 & 200 & 190 & 180 & 170 & 160 & 150 & 140 & 130 & 120 & 110 & 100 & 90 & 80 & 70 & 60 & 50 & 40 & 30 & 20 & 10 & 0 & -1
\end{tabular} 
${ }^{1} \mathrm{H}$ NMR (400 MHz, $\left.\mathrm{CDCl}_{3}\right)$ and ${ }^{13} \mathrm{C}$ NMR (101 MHz, $\left.\mathrm{CDCl}_{3}\right)$ of 7 Ph $\overbrace{\sim}^{\mathrm{OH}}$

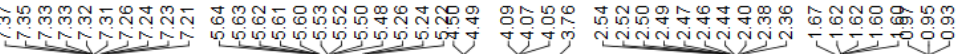

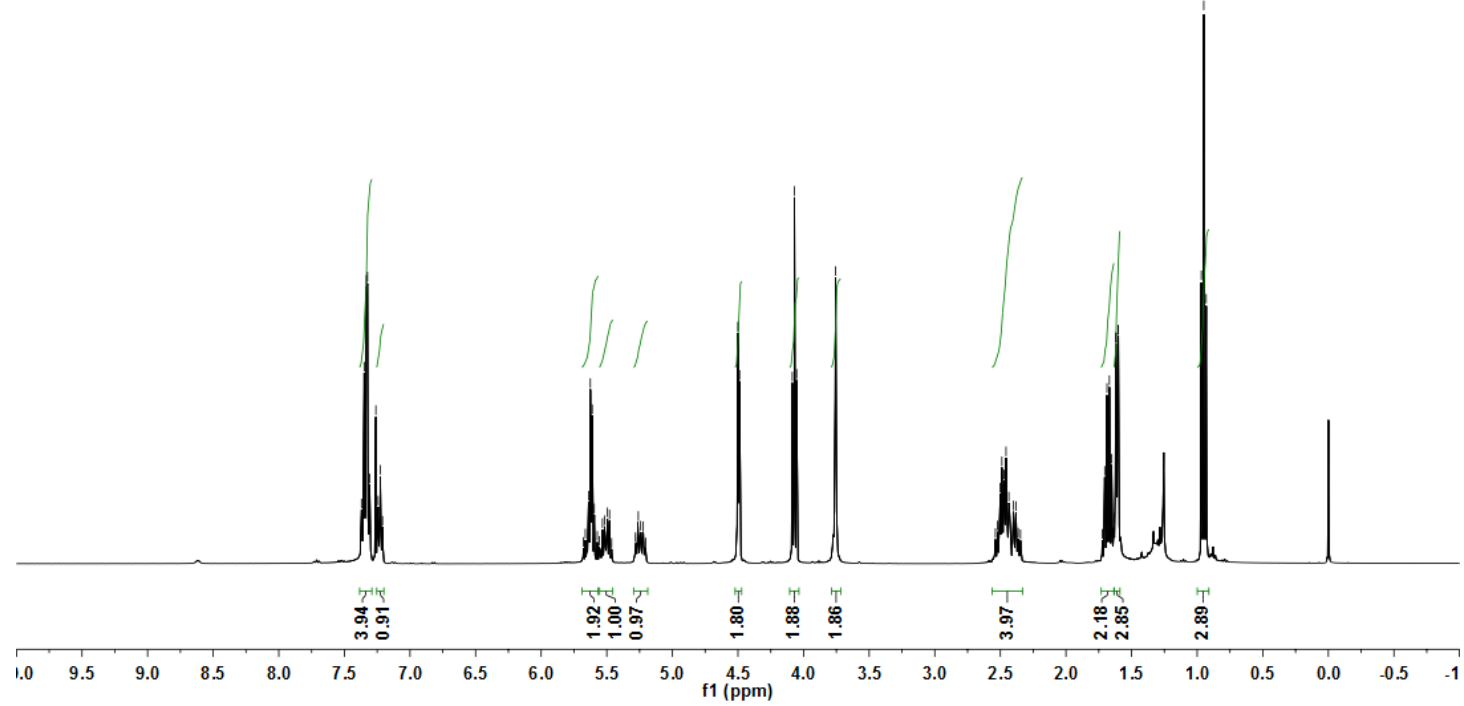

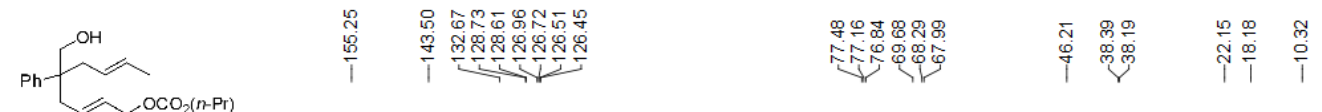

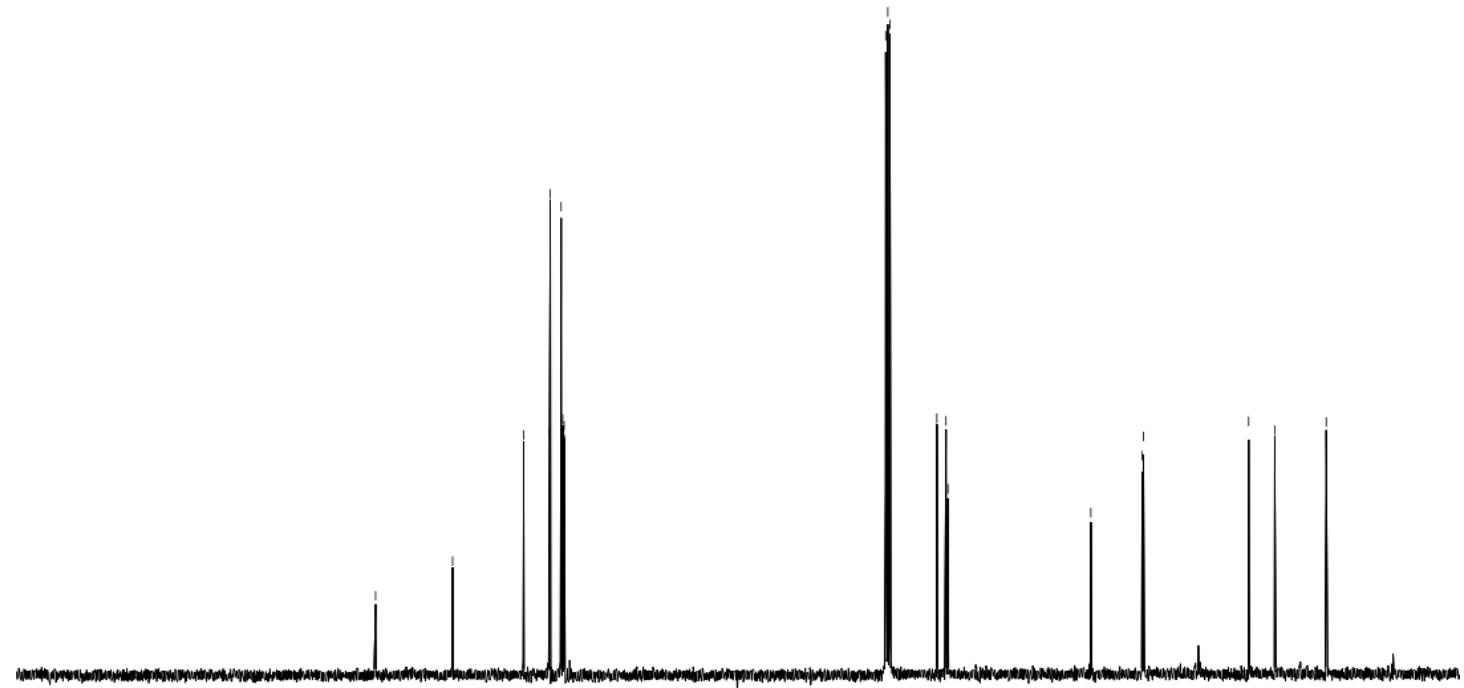

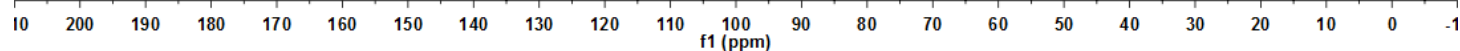


${ }^{1} \mathrm{H}$ NMR $\left(400 \mathrm{MHz}, \mathrm{CDCl}_{3}\right)$ and ${ }^{13} \mathrm{C}$ NMR $\left(101 \mathrm{MHz}, \mathrm{CDCl}_{3}\right)$ of 8

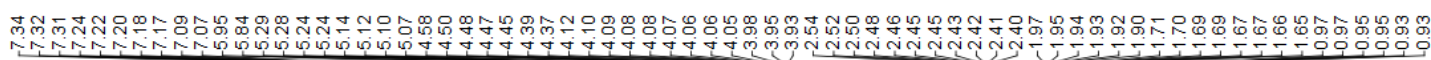
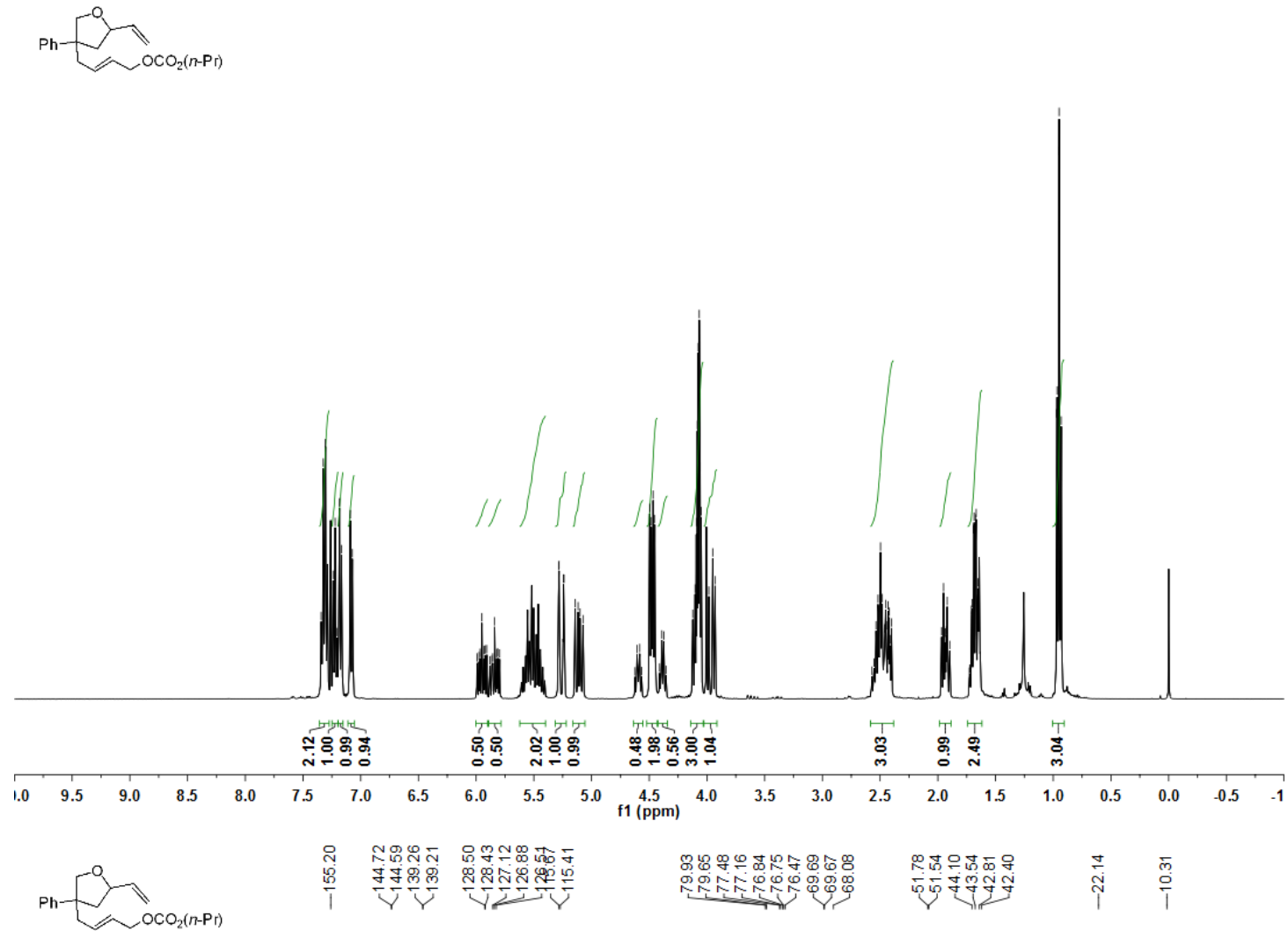

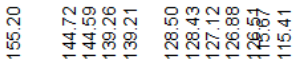

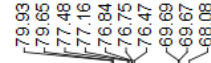

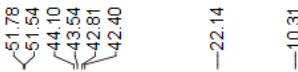

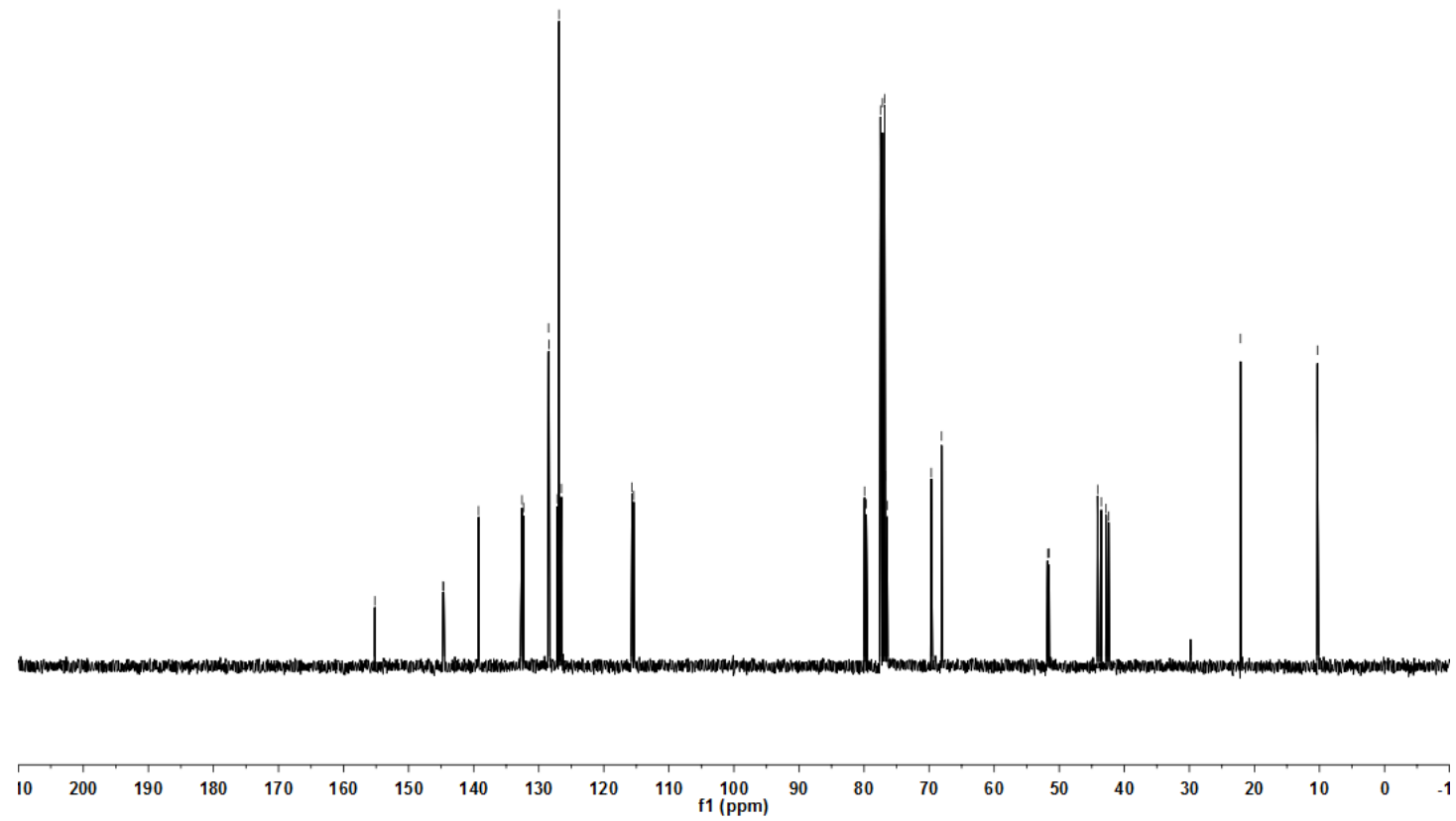


${ }^{1} \mathrm{H}$ NMR (400 MHz, $\left.\mathrm{CDCl}_{3}\right)$ and ${ }^{13} \mathrm{C}$ NMR $\left(101 \mathrm{MHz}, \mathrm{CDCl}_{3}\right)$ of 9
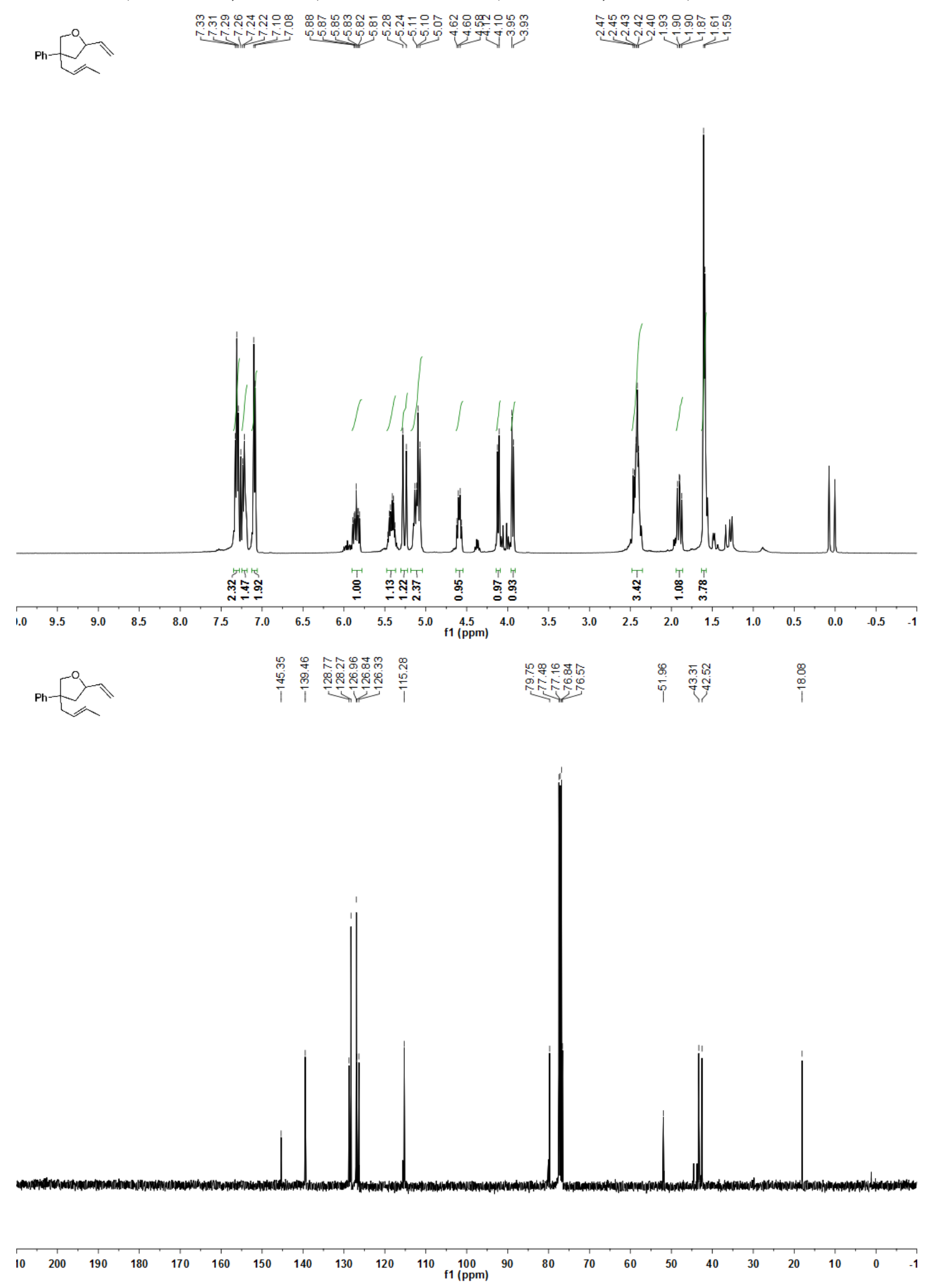
${ }^{1} \mathrm{H}$ NMR (400 MHz, $\left.\mathrm{CDCl}_{3}\right)$ and ${ }^{13} \mathrm{C}$ NMR (101 MHz, $\left.\mathrm{CDCl}_{3}\right)$ of 10

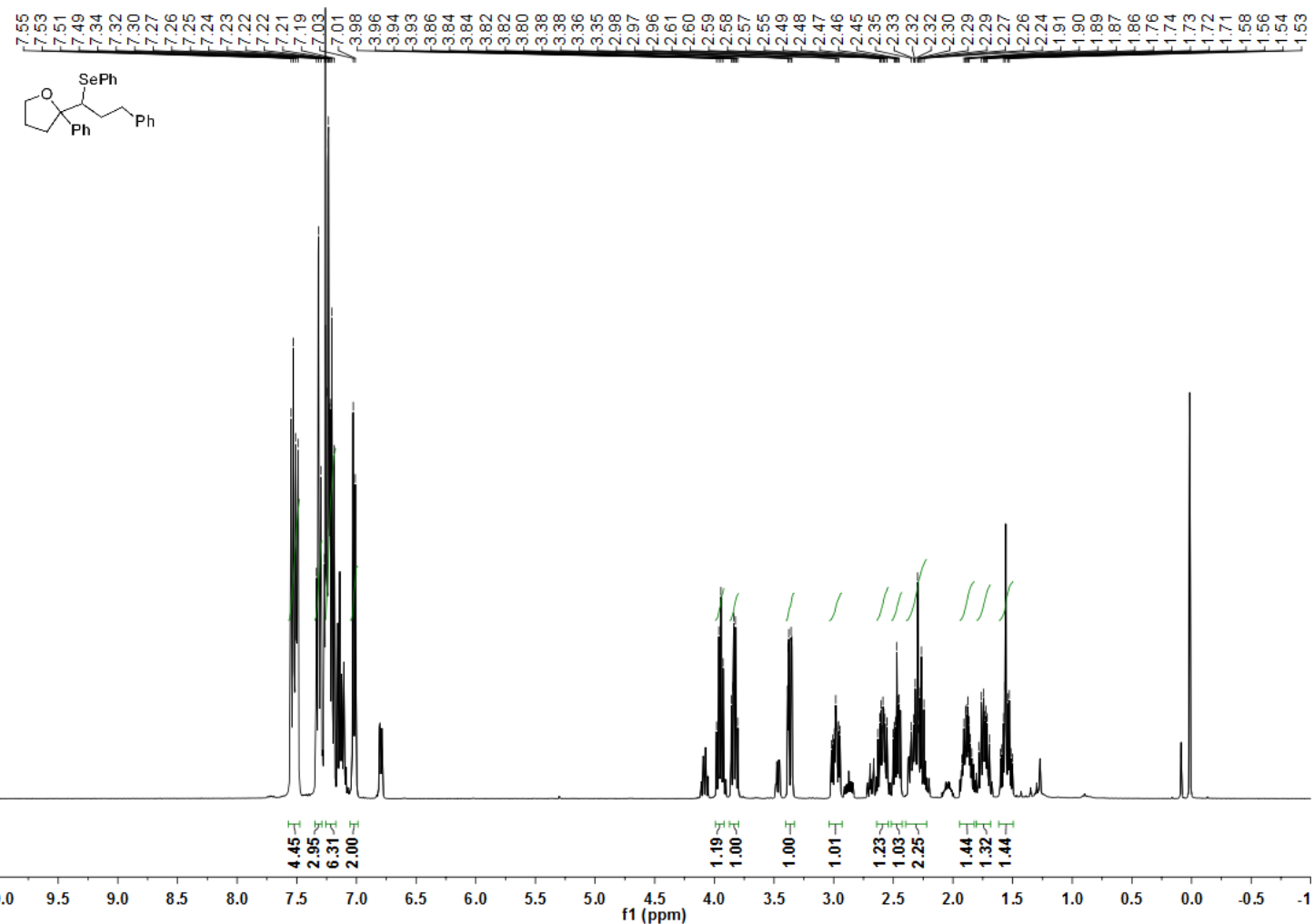

U

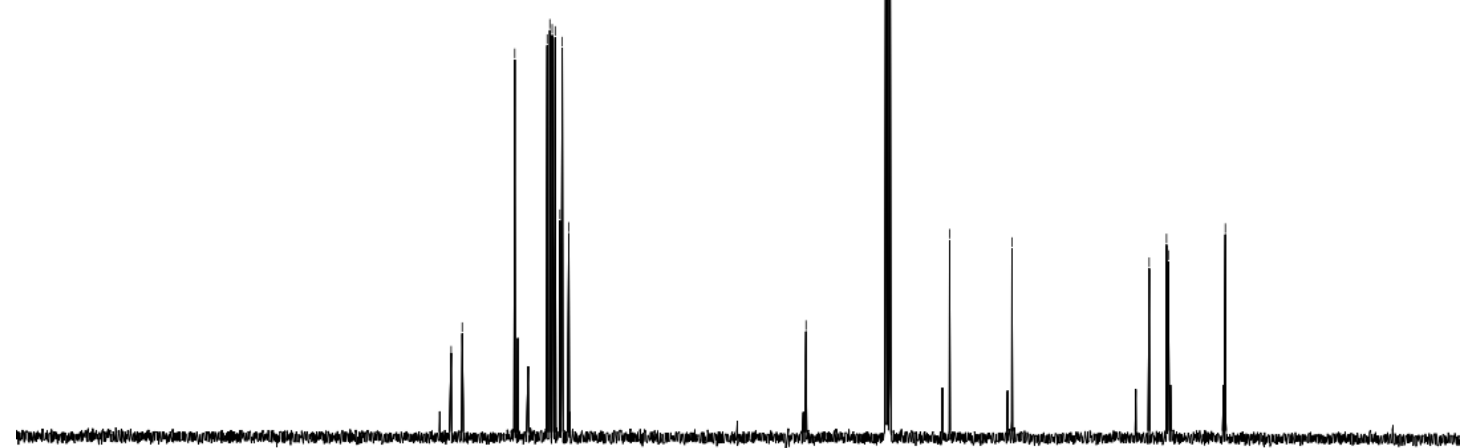

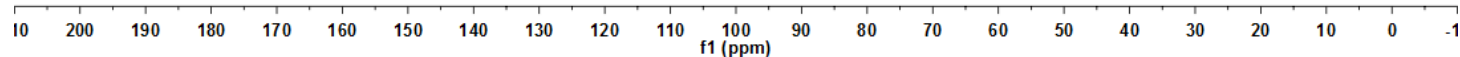

